EINAL

\title{
TECHNOLOGY DEVELOPMENT FOR COBALT F-T CATALYSTS
}

Contract No. DE-AC22-92PC92108

\section{QUARTERLY TECHNICAL PROGRESS REPORT No. 9}

Covering the Period October 1, 1994 to December 31, 1994

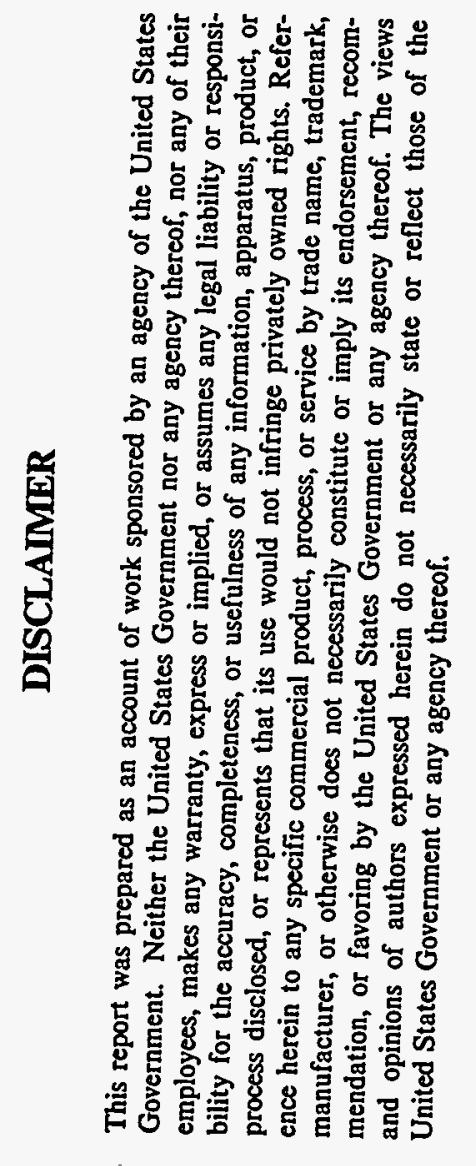

\author{
Prepared for \\ U. S. Department of Energy \\ Pittsburgh Energy Technology Center \\ PETC Project Manager: Richard E. Tischer \\ P. O. Box 10940 \\ Pittsburgh, PA 15236-0940 \\ Submitted by \\ Principal Investigator: Alan H. Singleton \\ Energy International Corporation \\ 135 William Pitt Way \\ Pittsburgh, PA 15238

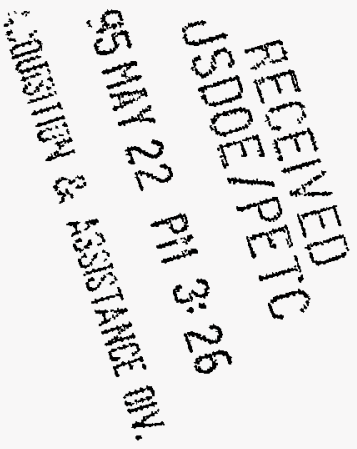

May 11, 1995 


\section{DISCLAIMER}

Portions of this document may be illegible in electronic image products. Images are produced from the best available original document. 


\section{TABLE OF CONTENTS} EXECUTIVE SUMMARY $\ldots \ldots \ldots \ldots \ldots \ldots \ldots \ldots \ldots \ldots$ iv

I. INTRODUCTION AND BACKGROUND $\ldots \ldots \ldots \ldots \ldots \ldots$

II. PROJECT DESCRIPTION $\ldots \ldots \ldots \ldots \ldots \ldots \ldots \ldots \ldots$

III. OBJECTIVES $\ldots \ldots \ldots \ldots \ldots \ldots \ldots \ldots \ldots \ldots \ldots \ldots \ldots \ldots \ldots \ldots$

IV. SUMMARY OF WORK ACCOMPLISHED THIS QUARTER $\ldots \ldots \ldots \ldots 4$

V. DETAILED DESCRIPTION OF TECHNICAL PROGRESS $\ldots \ldots \ldots \ldots 5$

A. Task 1: Catalyst Development $\ldots \ldots \ldots \ldots \ldots \ldots \ldots \ldots \ldots$

a. Technology Assessment $\ldots \ldots \ldots \ldots \ldots \ldots \ldots \ldots \ldots$

b. Catalyst Formulation $\ldots \ldots \ldots \ldots \ldots \ldots \ldots \ldots \ldots$

c. Catalyst Pretreatment $\ldots \ldots \ldots \ldots \ldots \ldots \ldots \ldots \ldots$

d. Catalyst Characterization $\ldots \ldots \ldots \ldots \ldots \ldots \ldots \ldots \ldots$

B. Task 2: Catalyst Testing $\ldots \ldots \ldots \ldots \ldots \ldots \ldots \ldots \ldots$

a. Fixed Bed Reactor $\ldots \ldots \ldots \ldots \ldots \ldots \ldots \ldots \ldots$

b. Slurry Bubble Column Reactor $\ldots \ldots \ldots \ldots \ldots \ldots \ldots \ldots$

VI. PLANS FOR THE NEXT REPORTING PERIOD $\ldots \ldots \ldots \ldots \ldots$

VII. ASSESSMENT OF PROSPECTS FOR FUTURE PROGRESS $\ldots \ldots \ldots \ldots 17$

VIII. APPENDICES $\ldots \ldots \ldots \ldots \ldots \ldots \ldots \ldots \ldots \ldots \ldots \ldots \ldots$
A. Co Catalyst Formulations
B. Fixed Bed Reaction Data
C. Chronology of Runs in Slurry Bubble Column Reactors
D. Slurry Bubble Column Data 


\section{LIST of TABLES}

Table No. Title

PageNa

1 List of Co-based F-T Catalysts Formulated $\ldots \ldots \ldots \ldots \ldots$

2 List of Water-Gas-Shift Catalysts and

F-T Catalysts with WGS Function $\ldots \ldots \ldots \ldots \ldots \ldots \ldots$

Summary of Physical Properties $\ldots \ldots \ldots \ldots \ldots \ldots . \ldots 24$

H2 Chemisorption and TPR Results $\ldots \ldots \ldots \ldots \ldots \ldots \ldots$

Summary of Fixed Bed Reaction Results . . . . . . . . . 31

6 Comparison of Conversion and Selectivity of Methane Reduction

Catalysts at Startup Conditions in M4-SBCR $\ldots \ldots \ldots \ldots \ldots \ldots$

$7 \quad$ Summary of M3-SBCR Run $\# 30$ Results $\ldots \ldots \ldots \ldots \ldots \ldots \ldots$

8 Summary of M4-SBCR Run $\# 24$ Results $\ldots \ldots \ldots \ldots \ldots \ldots \ldots$

9 Summary of M3-SBCR Run $\# 33$ Results $\ldots \ldots \ldots \ldots \ldots \ldots$

10 Summary of M4-SBCR Run $\# 26$ Results $\ldots \ldots \ldots \ldots \ldots \ldots$

11 Comparison of Conversion and Selectivity

Calsicat-Produced Catalysts at Startup Conditions in M4 \& M3 $\ldots \ldots 40$

12 Comparison of Conversion and Selectivity of MethaneReduction

Catalysts at Startup Conditions in M3 . . . . . . . . . . 41 


\section{ACKNOWLEDGEMENT}

The following persons contributed to this report: Ajay R. Belambe, Rachid Oukaci,

James J. Goodwin, Jr., and George Marcelin, all from University of Pittsburgh; William Gall, Alan Singleton, and Ronald W. Genser, from Energy International Corporation. 


\section{EXECUTIVE SUMMARY}

During the 9th quarter of the project, results have led to a number of important conclusions.

Vista $\mathrm{B} \gamma-\mathrm{Al}_{2} \mathrm{O}_{3}$ still seems to be the best alumina to use. A catalyst prepared by Calsicat using Condea alumina, which has a better initial particle size distribution for the SBCR requiring less sieving and discarding, was found to exhibit a much lower activity than a similar formulation using Vista B. The exact cause of this difference is not known since the Condea alumina has a suitable level of S impurities, surface area, and pore structure.

All catalysts prepared to date by Calsicat using Vista B have exhibited excellent particle size retention characteristics in the SBCR. Typical particle size reducctions have been in the range of $0.5-2.0 \%$.

SBCR trials made with a Co catalyst prepared by Calsicat, reduced, and coated with Soya confirmed that Soya/wax coating of the reduced catalysts does not protect the Co catalysts sufficiently from reoxidation to eliminate the need for gas phase reduction prior to SBC reaction. Co catalysts appear not to be able to be reduced suitablywhen suspended in a hydrocarbon liquid phase.

The optimum loading of $\mathrm{Zr}$ in Co/SiO ${ }_{2}$ catalysts in order to promote activity is on the order of $4 \mathrm{wt} \%$. Loadings as high as $15 \mathrm{wt} \%$ cause a significant reduction in overall activity. This has been confirmed by both fixed-bed and $\mathrm{SBC}$ reaction results. $\mathrm{La}$ promotion of $\mathrm{Co} / \mathrm{SiO}_{2}$ appears to give a rate enhancement similarily to $\mathrm{Zr}$ in the gas phase; however, promotion of $\mathrm{Co} / \mathrm{SiO}_{2}$ at the level of $8.5 \mathrm{wt} \% \mathrm{La}$ gave lower activity in the $\mathrm{SBCR}$ than $\mathrm{Co} / \mathrm{SiO}_{2}$ without La.

Catalyst pretreatment effects appear to be a function of preparation. For example, catalysts 
prepared using an organic solution seem to give higher activity when reduced directly and not calcined.

Co loadings of $30 \mathrm{wt} \%$ on alumina exhibit significantly higher activities than $20 \mathrm{wt} \%$ Co catalysts in excess of a linear increase. It is suggested that this is due to the fact that a greater percent of the additional Co is reducible to the metal. It is known that there is ca. $3 \mathrm{wt} \%$ of the Co which goes to form Co aluminates. Another significant portion of the Co is not able to be completely reduced due to interaction with the oxide support. However, these effects are mostly apparent in the initial $10 \mathrm{wt} \%$ Co loading.

Using a mixture of $\mathrm{Co} / \mathrm{Al}_{2} \mathrm{O}_{3}$ and a WGS catalyst results in higher activity and $\mathrm{Co}_{2}$ formation in the SBCR. Using $15 \mathrm{~g}$ of the Co catalyst and either 0,5 , or $15 \mathrm{~g}$ of $\mathrm{CuCr} / \mathrm{Al}_{2} \mathrm{O}_{3}$ produced $\mathrm{CO}$ conversions of $27.1,29.3$, and $35.8 \%$, respectively, and $\mathrm{CO}_{2}$ selectivities of $0.8,2.0$, and $6.3 \%$, respectively, at $240^{\circ} \mathrm{C}$.

Finally, significant work has focussed on pushing conversion to higher levels. This has been achieved by raising the temperature and lowering the flow rate. To date, the maximum conversion obtained has been $62.9 \%$ at $260^{\circ} \mathrm{C}$ and $540 \mathrm{SL} / \mathrm{h}\left(20 \% \mathrm{~N}_{2}\right.$ in a $\mathrm{H}_{2}+\mathrm{CO}$ mixture). However, low flow rates and higher temperatures result in greater heat being released than can be in many cases adequately removed since the SBCR does not have an internal heat exchanger. This results in difficulties in temperature control. In addition, the highly active and selective catalysts developed to date for use at lower temperatures start to lose activity above $240^{\circ} \mathrm{C}$. This loss of activity is especially great above $260^{\circ} \mathrm{C}$. 


\section{INTRODUCTION AND BACKGROUND}

The goal of this project is the development of a commercially viable, cobalt-based Fischer-Tropsch (F-T) catalyst for use in a slurry bubble column reactor. Cobalt-based catalysts have long been known as being active for F-T synthesis. They typically possess greater activity than iron-based catalysts, historically the predominant catalyst being used commercially for the conversion of syngas based on coal, but possess two disadvantages that somewhat lessen its value: (1) cobalt tends to make more methane than iron does, and (2) cobalt is less versatile with low $\mathrm{H}_{2} / \mathrm{CO}$ ratio syngas due to its lack of water-gas shift activity. Therefore, the major objectives of this work are (1) to develop a cobalt-based F-T catalyst with low (<5\%) methane selectivity, (2) to develop a cobalt-based F-T catalyst with water-gas shift activity, and (3) to combine both these improvements into one catalyst. It will be demonstrated that these catalysts have the desired activity, selectivity, and life, and can be made reproducibly. Following this experimental work, a design and a cost estimate will be prepared for a plant to produce sufficient quantities of catalyst for scale-up studies.

\section{PROJECT DESCRIPTION}

The Cobalt Fischer-Tropsch Catalyst Project is divided into five tasks designed to systematically develop catalysts through thorough investigation of influences of various promoters, additives, and supports on minimizing methane selectivity and increasing water-gas-shift activity.

Cobalt has long been known to be an excellent catalyst for the F-T synthesis. Nevertheless, all currently operating F-T plants feeding coal produced syngas use iron catalysts, in spite of the fact that cobalt-based catalysts have higher activity. Two factors that lower cobalt's value as a F-T catalyst are its poorer selectivity, that is, it produces more methane and its inability to be used with low 
$\mathrm{H}_{2} / \mathrm{CO}$ ratio syngas because of its lack of water-gas shift activity. The broad objective of this proposal is to overcome these deficiencies.

In pursuing F-T catalyst development, there are several aspects that need to be considered. These are catalyst formulation, catalyst pretreatment, and catalyst performance. All of these aspects will be dealt with in this project.

In broad terms, the technical approach that will be used is outlined below.

a. Conduct a thorough review of the literature on F-T synthesis, both the journal literature and the patent literature. In this review, identify approaches for improving methane selectivity of cobalt-based catalysts, identify additives that have WGS activity, identify catalyst formulation options, and define critical pretreatment parameters.

b. Based on the above review, develop a list of catalyst formulations with potential for low methane selectivity and a list of catalyst formulations with potential for promoting the WGS reaction.

c. Screen these catalysts in a small, fixed-bed reactor. If no catalyst meets the target specifications, go back to step b. Otherwise, run catalysts that meet discrimination criteria in a slurry bubble column reactor.

d. Once catalysts with low methane selectivity and WGS activity have been identified, develop a catalyst combining both these functions and test as described above.

e. Having found catalysts that meet the desired criteria for activity and selectivity, optimize the pretreatment conditions.

f. Demonstrate catalyst reproducibility by having a commercial subcontractor prepare multiple batches for testing. 
g. Demonstrate catalyst stability by running aging tests in a slurry bubble column reactor.

h. Based on above results, prepare a design for a plant to produce demonstration scale batches of catalyst. Develop capital and operating costs of this plant.

The program to carry out the above outlined work will consist of five major tasks:

Task 1 -- Catalyst Development

Task 2 - Catalyst Testing

Task 3 -- Catalyst Reproducibility Tests

Task 4 - Catalyst Aging Tests

Task 5 -- Preliminary Design and Cost Estimate for a Demonstration Scale Catalyst Production Facility

All aspects of the catalyst's role in F-T processing will be addressed, including catalyst preparation, pretreatment, and performance (activity, selectivity, and aging). In addition to gathering process data, the catalyst will be subjected to a number of analytical measurements at each stage to see how various treatments have affected the catalyst and its performance.

\section{OBJECTIVES}

The objective of this project is to investigate the influence of various promoters, additives, and supports on minimizing the methane selectivity and increasing the water-gas shift (WGS) activity of cobalt (Co) Fischer-Tropsch (F-T) catalysts. The ultimate goal of this investigation is to identify and demonstrate a catalyst preparation procedure that will be scaled up for the reproducible synthesis of commercial quantities of supported $\mathrm{CO}$ catalysts with desired activity, selectivity, and lifetime for use in F-T synthesis in three-phase slurry bubble column reactors. 


\section{SUMMARY OF WORK ACCOMPLISHED THUS OUARTER}

Seven (7) new catalysts were formulated and prepared during this period under both subtasks 1.2 and 1.3. Two more catalysts were prepared by Calsicat.

The characterization of all the catalysts in order to determine their physical properties (BET surface area, pore volume, pore size diameter, particle size distribution), as well as the cobalt reducibility, extent of reduction, and dispersion) was continued.

Fixed-bed reactor testing of the catalysts was continued. Six (6) new catalysts were tested for their F-T synthesis performance.

An investigation of the effect of pretreatment in various atmospheres (calcination in air or nitrogen prior to reduction in hydrogen, direct reduction without prior calcination, and reductionoxidation-reduction (ROR)) of a selected number of catalysts upon their performance for F-T synthesis was continued during this period.

A paper on "Zirconia Promotion of Fischer-Tropsch Cobalt catalysts: Behavior in Fixed-Bed and Slurry Bubble Column Reactors" was submitted for the preprints of the 209th ACS National Meeting, Division of Fuel Chemistry, Symposium on Synthesis Gas Chemistry, April 2-6, 1995 in Anaheim, CA.

Under subtask 2.2 during this reporting period a total of 11 runs were made in the two slurry bubble column reactors with eleven catalysts, including five on alumina, two from Calsicat, one WGS blend, and three on silica support. Four high CO conversion runs were made. Data were compiled to compare the $\mathrm{CO}$ conversions and product selectivities of the-methane reduction catalysts. 


\section{DETAILED DESCRIPTION OF TECHINICAL PROGRESS}

\section{A. TASK 1: CATALYST DEVELOPMENT}

\section{a. Technology Assessment (Subtask 1.1)}

Except for regular updating of the literature with the most recent publications, there was no, activity during this period to report in this subtask.

\section{b. Catalyst Formulation (Subtasks 1.2, 1.3, 1.4)}

An updated list of all the catalysts formulated so far within Subtask 1.2 (including those catalysts listed in previous quarterly reports) is given in Table 1 with their compositions. The last column of Table 1 indicates whether the catalyst has already been prepared. Out of the 78 catalysts listed in Table 1, two (CAL.09-CAL.10) were prepared by Calsicat. Seven (7) new Co catalysts (highlighted in Table 1) were formulated and prepared during this period. The detailed formulations of the new catalysts are given in Appendix A.

Co.057, is similar to Co.055, with $\mathrm{SiO}_{2}$ as the support instead of $\mathrm{Al}_{2} \mathrm{O}_{3}$. Co. 058 and $\mathrm{Co} .059$, both alumina-supported catalysts, are similar to $\mathrm{Co} .055$ and Co.015, but without Re or Ru promoters. Co.063, also an alumina-supported catalyst, is similar to Co.034, but with $\mathrm{La}_{2} \mathrm{O}_{3}$ replacing $\mathrm{ZrO}_{2}$ as the oxide promoter. These four catalysts were formulated in order to investigate further the effects of $\mathrm{La}_{2} \mathrm{O}_{3}$ on the catalytic properties of $\mathrm{Co} . \mathrm{La}_{2} \mathrm{O}_{3}$ addition was found in previous formulations to enhance significantly the activity of cobalt for F-T synthesis.

Co.060-Co.062, all alumina-supported catalysts, were formulated with $30 \%$ Co loading in order to achieve higher conversion levels in the slurry bubble column reactor.

CAL.09-CAL.10 were formulated with the same composition as that of $\mathrm{Co} .047$ which was selected as the low methane catalyst for the reproducibility test. They were both prepared in small 
batches for formulation check and determination of the best pretreatment to adopt for the preparation of the larger batches to be used for the reproducibility tests. CAL.09 was calcined in nitrogen, reduced in hydrogen, and protected in soya instead of the paraffin wax used in some of the previous samples (CAL.07 and CAL.08). CAL.10 was prepared with a different alumina (Condea) than the one (Vista-B) used so far during this project. The Condea alumina was selected for its narrow particle size distribution in the range required for slurry bubble column applications.

Under Subtask 1.3 five (5) new Co catalysts with a water-gas shift component were prepared. CoW.08 and CoW.09 are similar to catalysts which had been prepared previously, but with different loadings of the various metals. CoW. 08 , similar to CoW. 05 , but with $30 \%$ Co, was prepared by pre-impregnating the alumina with $\mathrm{Cu}-\mathrm{CrO}$ and calcining this support precursor at 750 ${ }^{\circ} \mathrm{C}$, before incorporating the cobalt. CoW.09, similar to $\mathrm{CoW} .06$, but with $10 \% \mathrm{Cu}$ and $8 \% \mathrm{Cr}$, was prepared in a similar way. CoW10-CoW12 are all Co-Fe based catalysts with other promoters $(\mathrm{Ru}$ and $\mathrm{K}$ ) on either alumina or silica. The list of these catalysts is given in Table 2 with their composition, and the detailed formulations are included in Appendix A.

\section{c. Catalyst Pretreatment (Subtask 1.5)}

The investigation of the effects of pretreatment on the catalytic properties of a selected number of catalysts (Co.004, C0.015, Co.017, Co.053, Co.055 and Co.056) was continued during this period. Except for $\mathrm{Co} .053$, all the catalysts were promoted with $\mathrm{La}_{2} \mathrm{O}_{3}$ and $\mathrm{Ru}$ or $\mathrm{Re}$ in the case of Co.055. The effects of pretreatment in various atmosphere (calcination in air or in nitrogen prior to reduction in $\mathrm{H}_{2}$ direct reduction in $\mathrm{H}_{2}$ without prior calcination, and reduction-oxidation-reduction (ROR)) were addressed. The results are included in subtask 2.1 which summarizes the results 
obtained when these catalysts were tested in the fixed bed reactor.

\section{d. Catalyst Characterization (Subtask 1.6)}

(1) Physical Properties

Table 3 gives an update on the physical property measurements carried out so far. Nitrogen physisorption at $77 \mathrm{~K}$ was used to obtain surface area, pore volume and pore diameter of the calcined catalysts. Average particle size of the catalysts was determined using a Microtrac particle size analyzer.

(2) Static Hydrogen Chemisorption

An updated summary of the properties of the Co catalysts as determined by $\mathrm{H}_{2}$ chemisorption is given in Table 4. Metal dispersions and particle sizes of CAL.09 and CAL.010 were determined by $\mathrm{CO}$ chemisorption carried out at Calsicat. Co.004, Co.015, Co.053, and Co.055 were characterized by static hydrogen chemisorption at the University of Pittsburgh following different pretreatments.

(3) Temperature Programmed Reduction (TPR)

TPR experiments were carried out in an automated Altamira Instruments AMI-1 system. The procedure for these measurements was described in a previous quarterly report. All the TPR results obtained so far are summarized in Table 4. 


\section{B. TASK 2: CATALYST TESTING}

a. Subtask 2.1 - Fixed Bed Reaction Studies:

\section{E-T Synthesis}

The reaction conditions and procedure were described in previous quarterly reports. Six (6) new catalysts (Co.044, Co.045, Co.056, Co.057, Co.060, CAL.10) were tested for the first time during this reporting period. Catalysts Co.004 (run \#6-8), Co.015 (run \#3), Co.017 (run \#3), Co. 053 (run \#4-6), and Co.055 (run \#4-7) were also retested following the different pretreatment procedures described in Subtask 1.5.

The performances of all these catalysts (highlighted in Table 5) at steady state are compared in Table 5 with those reported in the previous quarterly report. All the pertinent data (CO conversion, rates, product distributions, chain growth probabilities, Anderson-Schulz-Flory distributions, time-on-stream activity and Arrhenius plots when available) obtained with each run for each catalyst is given in Appendix B.

Co.044 and Co.045 were tested in order to investigate the effect of $\mathrm{Zr}$ loading on the catalytic properties of promoted $\mathrm{Co} / \mathrm{SiO}_{2}$ catalysts. These results and those obtained previously with similar formulations but different $\mathrm{Zr}$ loadings, indicate that $\mathrm{Zr}$ enhanced the activity of cobalt for $\mathrm{F}-\mathrm{T}$ synthesis when it is pre-impregnated on the support. However, $\mathrm{Zr}$ loadings greater than $4 \mathrm{wt} \%$ did not seem to provide any significant effect beyond what was observed at the $4 \%$ level. Co. 045 with its $15 \mathrm{wt} \%$ of $\mathrm{Zr}$ showed even lower activity for $\mathrm{F}-\mathrm{T}$ synthesis than the catalysts containing 4 (Co.045) or $8.5 w t \%(C o .024)$ of $\mathrm{Zr}$.

Co.056 and $\mathrm{Co} .057$, both $\mathrm{SiO}_{2}$-supported catalysts, were tested in order to investigate the effect of $\mathrm{La}_{2} \mathrm{O}_{3}$ promotion on the catalytic properties of Co. The fixed-bed reactor results show that 
$\mathrm{La}_{2} \mathrm{O}_{3}$ promotion had a similar enhancing effect on the activity of $\mathrm{Co} / \mathrm{SiO}_{2}$ catalysts for $\mathrm{F}-\mathrm{T}$ synthesis to the effect observed with $\mathrm{Zr}$ promotion.

Co.060 was tested as part of an ongoing study of the effect of Co loading. The preliminary results indicate that, as the Co loading is increased beyond the $20 \mathrm{wt} \%$ level used in most of the catalysts formulated so far in this project, the additional cobalt interacts less with the support, thus resulting in a higher rate of syngas conversion per unit weight of Co.

Co.004, Co.015, Co.017, Co.053 and Co.055 were retested following various pretreatments prior to the standard reduction and reaction procedures. The results indicate that not only the pretreatment environment but also the preparation method (aqueous vs organic) may play a major role in determining the activity of these catalysts. For the catalysts prepared entirely by aqueous impregnation, the pretreatment environment (air, nitrogen, hydrogen, or ROR) did not seem to have any effect on the catalysts activity. However, when at least on step in the catalyst preparation involved an impregnation with an organic solution of one of the metal precursors, the catalyst exhibited a very high activity when it was directly reduced without prior calcination.

Finally, the last trial catalyst prepared at Calsicat, CAL.10, was also tested using the standard reaction conditions. It had a lower activity than that obtained with similar catalysts prepared with Vista-B alumina (e.g. CAL.05 or Co.047). The reason for such a behavior is not yet understood.

\section{b. Subtask 2.2 - Slurry Bubble Column Testing ("SBCR")}

\section{Run Chronolgy}

During this reporting period a total of 11 runs were performed in the SBCR's, Runs M3-30 through 34 and M4-22 through 27. A chronology of the experimental runs performed in the two 
slurry bubble column reactors - M3 and M4 - is given in Appendix C. All comparisons of CO conversions and $\mathrm{CH}_{4}$ selectivities discussed in this chronology were obtained from results obtained at the initial startup conditions, i.e. $240^{\circ} \mathrm{C}$ temperature, 450 psi pressure, and $2 / 1 \mathrm{H}_{2} / \mathrm{CO}$ feed gas ratio.

A complete summary of all runs made in the M3-SBCR is given in Table I in Appendix D. Four tables of data were prepared in order to compare catalyst activities for Runs 30 to 34 in M3SBCR at the same run conditions (see Tables II, III, IV, and V in Appendix D).

A complete summary of all runs made in the M4-SBCR are given in Table VI, Appendix D. Four tables were prepared to compare catalyst activities for Runs 22 to 27 in M4-SBCR at the same run conditions (see Tables VII, VII, IX, and X in Appendix D).

2. Discussion of Results

(a) Water-Gas Shift Activity Test

Run 22 was made in the M4-SBCR to evaluate the effect of adding a blend of equal amounts of two catalysts: 15.5 gm of Catalyst No. WGS.09 (water-gas shift catalyst) and 15.0 gm of Catalyst No. Co.005 (methane reduction). The $\mathrm{CO}$ conversion at startup conditions was considerably higher than observed with Catalyst Blend A in Run 14 in M4-SBCR, 35.6\% vs. 29.3\% (see Table 6). Also, the $\mathrm{CO}_{2}$ selectivity was much higher, $6.3 \%$ vs. $2.0 \%$. Some water-gas shift activity has occurred with this catalyst blend as demonstrated by the higher $\mathrm{CO}_{2}$ yield and increase in $\mathrm{CO}$ conversion. Water-gas shift activity was also observed at all other run conditions.

(b) High CO Conversion Runs

Four high $\mathrm{CO}$ conversion runs were made during this reporting period. Three runs were made with a larger charge of catalyst, approximately $30 \mathrm{gm}$ of Catalyst No. CO.053, and one run was made 
with a normal charge of $15 \mathrm{gm}$ of CALSICAT Catalyst No. CAL.10.

Run No. 30 in the M3-SBCR was started with a charge of $29.6 \mathrm{gm}$ of Catalyst No. Co.053. The CO conversion at startup conditions was $41.8 \%$, the same as observed in Run 29 . CO conversion increased to $46.9 \%$ at $260^{\circ} \mathrm{C}$, but dropped to $41.4 \%$ at $280^{\circ} \mathrm{C}$. The $\mathrm{CO}$ conversion did not change even at temperatures up to $320^{\circ} \mathrm{C}$. The run was terminated when the gas preheater plugged when the heater temperature reached $550^{\circ} \mathrm{C}$. It is speculated that the catalyst activity began to decrease above $260^{\circ} \mathrm{C}$. Both recovered catalysts from Runs 29 and 30 contained small amounts of iron that came from carbonyls in the $\mathrm{CO}$ feed gas. The lead oxide filtration beds need to be changed more frequently to prevent iron from poisoning the catalysts. A summary of the data for Run 30 is given in Table 7.

Run No. 24 in the M4-SBCR was started with 28.5 gm of Catalyst No. Co.053, see Table 8. The CO conversion at startup conditions was $50.4 \%$, which was the highest conversion observed to date. Raising the temperature to $260^{\circ} \mathrm{C}$ increased the $\mathrm{CO}$ conversion only to $55.3 \%$ (see Period 3). Lowering the total gas rate from 900 to 540 standard liters per hour ("SLH") with $20 \% \mathrm{~N}_{2}$ in the feed gas yielded a CO conversion of $62.9 \%$ with a methane selectivity of $23.2 \%$. At this point, the reactor temperature could not be controlled since there is no way to remove the heat of reaction from inside the SBCR reactor because there is no internal heat removal device. The heat of reaction can be removed only by lowering the feed gas temperature or increasing the inert gas $\left(\mathrm{N}_{2}\right)$ flow or some combination of both.

Run No. 33 in the M3-SBCR was started with $31.4 \mathrm{gm}$ of a third batch of Catalyst No. Co.053. The CO conversion at startup conditions was $41.4 \%$, which was the same as Runs 29 and 30 in the M3-SBCR. Several run periods were made in which the reactor pressure was lowered to 
$300 \mathrm{psi}$, the total feed gas rate was lowered to $400 \mathrm{SLH}$, and finally the reactor temperature was raised to $260^{\circ} \mathrm{C}$ (see Table 9). The $\mathrm{CO}$ conversion reached a maximum of only 49.2\%. The conversion dropped to $16.1 \%$ when the reactor was returned to startup conditions. It is believed that most of the catalyst activity was lost at the higher temperature, $260^{\circ} \mathrm{C}$. Alumina based catalysts have been found to be more sensitive to higher temperatures.

Run No. 26 in the M4-SBCR was started with a charge of 15.6 gm of Catalyst No. CAL.10. This catalyst which was received from Calsicat contained $20 \mathrm{wt} \%$ Co with $0.5 \% \mathrm{~K}$ and $0.5 \% \mathrm{Ru}$ on Condea alumina. The initial CO conversion was only $21.9 \%$ which was $5 \%$ lower than other Calsicat catalysts made with Vista B alumina support. Several run periods were made to investigate the effect of lowering the total gas flow from 900 to 436 SLH and nitrogen concentration in the feed gas from $60 \%$ to $6 \%$ (see Table 10). The $\mathrm{CO}$ conversion decreased rather than increased as expected. This run will be repeated with a larger amount of a more active catalyst.

A lot has been learned from these five runs about obtaining high $\mathrm{CO}$ conversion levels. To obtain high conversions in the SBCR, the following recommendations should be investigated:

o Operate at the lowest total gas feed rate that can keep the catalyst suspended.

- Operate at the lowest $\mathrm{N}_{2}$ feed rate. Some $\mathrm{N}_{2}$ is necessary since it is used as an internal standard for calculating conversions.

o Investigate higher cobalt loading on the catalyst.

o Increase catalyst loading in the SBCR.

o Determine maximum reaction temperature where catalyst begins to deactivate.

Further attempts to operate at higher $\mathrm{CO}$ conversions will be made during the next reporting 
period using the above recommendations.

(c) Calsicat Catalyst Test Run

One run was made with a catalyst supplied by Calsicat that was hydrogen reduced and coated with Soya. The CO conversion at the initial startup conditions was only $17.4 \%$ (see Run 23 in M4SBCR of Table 11). All of the wax coated catalysts supplied by Calsicat have shown low catalyst activity.

(d) Miscellaneous Runs Made to Evaluate the Effects of Various Promoters

One run was made with a catalyst similar to base case Catalyst No. Co.001 (20\% Co, $1 \% \mathrm{Re}$, $1 \% \mathrm{La}_{2} \mathrm{O}_{3}$, plus $0.13 \% \mathrm{~K}$ on alumina) but without $\mathrm{K}$ (see Run 31 in M3-SBCR in Table 12). The CO conversion was high, $30 \%$ as expected, but the $\mathrm{CH}_{4}$ selectivity was also high, $23.7 \%$. The addition of $\mathrm{K}$ should reduce the methane selectivity, but would also reduce catalyst activity as reported previously.

A run was made with a catalyst containing $20 \%$ cobalt plus $8.5 \% \mathrm{La}_{2} \mathrm{O}_{3}$ on silica support. The initial CO conversion was only $11.4 \%$, see Run 25 in M4-SBCR in Table 6 . The addition of a large amount of $\mathrm{La}_{2} \mathrm{O}_{3}$ had a negative effect on the catalyst activity, reducing the $\mathrm{CO}$ conversion about $7 \%$ (see Run No. 9 in M3 on Table 12).

One run was made to determine the effect of adding a larger amount of $\mathrm{Zr}$ on a cobalt-silica catalyst. Using a $15 \% \mathrm{Zr}$ promoted catalyst yielded a $21 \% \mathrm{CO}$ conversion compared to $25 \% \mathrm{CO}$ conversion with an $8.5 \% \mathrm{Zr}$ promoted catalyst, and $23.2 \% \mathrm{CO}$ conversion with $0.7 \% \mathrm{Zr}$ promoter (see Runs 17, 19, and 32 in M3-SBCR, Table 12). Adding a larger amount of $\mathrm{Zr}$ decreased catalyst activity. The optimum concentration of $\mathrm{Zr}$ needed can be determined by testing additional catalysts with $\mathrm{Zr}$ concentrations in the range of $0.7 \%$ to $15 \mathrm{wt} \%$. 
Another run was made to evaluate the effect of $\mathrm{K}$ concentration on catalyst activity. Catalyst No. Co. 050 which contained $20 \% \mathrm{Co}, 0.5 \% \mathrm{Ru}, 8.5 \% \mathrm{Zr}$, and $0.1 \% \mathrm{~K}$ on silica yielded a $\mathrm{CO}$ conversion of $20.4 \%$. The $\mathrm{CO}$ conversion and $\mathrm{CH}_{4}$ selectivity were the same as obtained with Catalyst Co.048 that contained $0.3 \% \mathrm{~K}$, see Runs 9 and 27 in the M4-SBCR on Table 6 . The optimum amount of $\mathrm{K}$ to add for methane reduction without significantly lowering catalyst activity is somewhere between 0.1 and $0.3 \mathrm{wt} \%$. Adding $0.5 \% \mathrm{~K}$ decreased catalyst activity significantly, see

\section{Run 7 in Table 6.}

The last catalyst tested this period was a $30 \mathrm{wt} \%$ Co on alumina support with no additives or promoters. The $\mathrm{CO}$ conversion was high, $31.4 \%$, but the $\mathrm{CH}_{4}$ selectivity was also high, $14 \%$, which is also in the expected range (see Run 34 in M3-SBCR on Table 12). This will be used as a basis for comparing similar catalysts with various additives and promoters.

\section{Catalyst Recovery Analyses}

The catalysts charged for all runs, except for Run 20 in M3 and in M4, have been recovered and the particle size distributions have been measured. The charge and recovered weights of all catalysts are given in Table 13. The charge weights are in the $\mathrm{H}_{2}$ reduced state while the recovered weights are in the oxidized state. Taking this into account, catalyst recoveries are quite good (greater than $90 \%$ ). The mean volumetric diameters of both the charged and recovered catalysts with the calculated percent reduction in particle size are also given in Table 13. The particle size at the lower $10 \%$ pass-through point of the sample for both the charged and recovered catalysts are also given in Table 13.

A comparison of the mean volume diameters of the total feed and recovered samples shows that most of the alumina supported catalysts exhibited the least amount of particle size attrition ( 5 to 
$10 \%$ ) during the reaction. The silica supported catalysts showed a 10 to $20 \%$ particle size reduction while a titanium supported catalyst had the highest reaction, $18.2 \%$ (see Run 8 in the M4-SBCR in Table 13).

Seven catalysts prepared by Calsicat have been tested in the SBCR and the particle size reduction was very low, $0.5 \%$ for CAL. 08 and $1.6 \%$ for CAL .05 . All these catalysts were prepared on a Vista B gamma-alumina catalyst support which has shown good resistance to attrition in the SBCR's.

If you compare the particle size of each sample of the $10 \%$ pass-through point, the increase in the amount of fines found in the recovered catalyts, as indicated by the lower particle size at the $10 \%$ point, is nearly proportional to the reduction in particle size obtained by comparing the mean volume diameters of the charge vs. recovered catalysts (see Table 13). Since the particle size of the charge catalyst was determined on the catalyst after hydrogen reduction and air oxidation, we know that attrition occurred only during the SBCR reaction.

\section{PLANS FOR THE NEXT REPORTING PERIOD}

Several tasks are planned for the next reporting period:

A. The fixed-bed reactor testing of new catalysts will be continued. The fixed-bed reactor testing of the series of $\mathrm{Al}_{2} \mathrm{O}_{3}$ - and $\mathrm{SiO}_{2}$ supported Co catalysts with a water-gas shift function will be continued.

B. The systematic characterization of all the catalysts will be continued using the following techniques:

- Selective hydrogen chemisorption on the reduced catalysts at $100^{\circ} \mathrm{C}$; 
- X-ray diffraction before reduction, after reduction, and after slurry bubble column reaction;

- Temperature programmed reduction (TPR);

- Temperature programmed desorption (TPD).

C. New catalyst formulations will be generated in order to pursue the effect of selectivity promoters.

D. The investigation of the effect of pretreatment conditions including calcination and reduction will be completed.

E. Additional runs will be made in the SBCR's to maximize the CO conversion.

F. Reproducibility tests and aging runs will be made on catalyst obtained from Calsicat.

G. Several catalyst formulations and mixtures will be tested for water-gas shift activity. 
VII. ASSESSMENT OF PROSPECTS FOR FUTURE PROGRESS

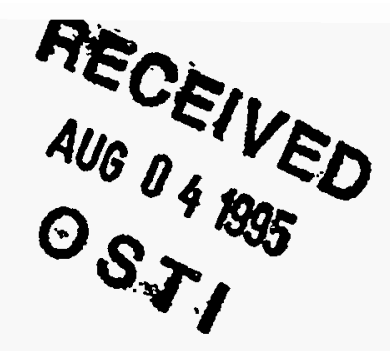

The technical approach which has been proposed remains the same and all the tasks are proceeding within schedule. 
Table 1. List of Co-based FT Catalysts Formulated

\begin{tabular}{|c|c|c|c|c|c|c|}
\hline aANo.\% & $\% \% \% \mathrm{Co}^{\prime}$ & \%x & $\% \%$ prom $\%$ & 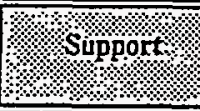 & 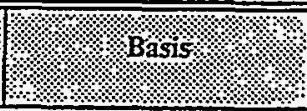 & Prepar \\
\hline Co.001 & $20 \%$ & $1 \% \operatorname{Re}$ & $\begin{array}{l}1 \% \mathrm{La}_{2} \mathrm{O}_{3} \\
0.13 \% \mathrm{~K}\end{array}$ & $\gamma$-alumina & $\begin{array}{l}\text { U.S. Pat. } \\
4,880,763\end{array}$ & $\mathbf{P}$ \\
\hline Co.002 & $20 \%$ & $0.43 \% \mathrm{Ru}$ & $1 \% \mathrm{~L}_{3}, \mathrm{O}_{3}$ & $\gamma$-alumina & $\begin{array}{l}\text { U.S. Pat. } \\
4,413,064\end{array}$ & $\mathbf{P}$ \\
\hline Co.003 & $20 \%$ & $0.5 \% \mathrm{Ru}$ & $1 \% \mathrm{~L}_{2} \mathrm{O}_{3}$ & $\gamma$-alumina & $\begin{array}{l}\text { U.S. Pat. } \\
4,413,064\end{array}$ & $P$ \\
\hline Co.004 & $20 \%$ & $0.43 \% \mathrm{Ru}$ & $1 \% \mathrm{~L}_{3} \mathrm{O}_{3}$ & $\gamma$-alumina & Reproduce Co.002 & $\mathbf{P}$ \\
\hline Co.005 & $20 \%$ & 0 & 0 & y-alumina & Base Catalyst & $\mathbf{P}$ \\
\hline Co.005A & $20 \%$ & $0.4 \% \mathrm{Ru}$ & 0 & $\gamma$-alumina & $\begin{array}{l}\text { Ru added to calcined } \\
\text { Co.005 by IW } \\
\text { impreg. }\end{array}$ & $\mathbf{P}$ \\
\hline Co.006 & $12 \%$ & $0.75 \% \operatorname{Re}$ & 0 & Titania & $\begin{array}{l}\text { U.S. Pat } \\
4,794,009\end{array}$ & $\mathbf{P}$ \\
\hline Co.007 & $20 \%$ & 0 & 0 & Titania & Base Catalyst & $\mathbf{P}$ \\
\hline Co.008 & $20 \%$ & 0 & 0 & Silica & $\begin{array}{c}\text { UK Pat Appl. } \\
\text { GB } 2125062 \mathrm{~A} \\
\text { (Kneaded with excess } \\
\text { liquid) }\end{array}$ & $\mathbf{P}$ \\
\hline Co.009 & $20 \%$ & $0.5 \% \mathrm{Ru}$ & 0 & $\gamma$-alumina & $\begin{array}{c}\text { Ru-Promoted Catalyst } \\
\text { (use Ru Chloride, } \\
\text { single-step aquaous } \\
\text { IW) }\end{array}$ & $\mathbf{p}$ \\
\hline Co.010 & $20 \%$ & $\mathbf{0}$ & 0 & $\gamma$-alumina & $\begin{array}{l}\text { Base Catalyst } \\
\text { (non-calcined) }\end{array}$ & $\mathbf{P}$ \\
\hline Co.010A & $20 \%$ & $0.4 \% \mathrm{Ru}$ & 0 & $\gamma$-alumina & $\begin{array}{l}\text { Ru added to dried } \\
\text { Co.010 by IW } \\
\text { impreg. }\end{array}$ & $\mathbf{P}$ \\
\hline Co.011 & $20 \%$ & 0 & 0 & Silica & $\begin{array}{l}\text { UK Pat Appl. } \\
\text { GB } 2125062 \text { A } \\
\text { (Kneaded) }\end{array}$ & $\mathbf{P}$ \\
\hline Co.012 & $20 \%$ & 0 & 0 & Silica & $\begin{array}{r}\text { Base Catalyst } \\
\text { (Inc. Wetness) }\end{array}$ & $\mathbf{P}$ \\
\hline Co.013 & $20 \%$ & 0 & 0 & Titania & $\begin{array}{c}\text { Base Catalyst } \\
\text { like Co.007, but all } \\
\text { aqueous }\end{array}$ & $\mathbf{P}$ \\
\hline Co.014 & $12 \%$ & $0.5 \% R u$ & 0 & Titania & $\begin{array}{c}\text { Ru-Promoted Catalyst } \\
\text { (aqueous IW co- } \\
\text { impregnation) }\end{array}$ & $\mathbf{P}$ \\
\hline Co.015 & $20 \%$ & $0.43 \% \mathrm{Ru}$ & $1 \% \mathrm{La}_{2} \mathrm{O}_{3}$ & $\gamma$-Alumina & $\begin{array}{c}\text { Similar to Co.002, } \\
\text { but all aqueous }\end{array}$ & $\mathbf{P}^{\circ}$ \\
\hline Co.016 & $20 \%$ & $0.43 \% \mathrm{Ru}$ & $1 \% \mathrm{La}_{2} \mathrm{O}_{3}$ & $\gamma$-Alumina & $\begin{array}{c}\text { Similar to Co.015, } \\
\text { but calcined after Co } \\
\text { impregnation }\end{array}$ & $\mathbf{P}$ \\
\hline
\end{tabular}


Table 1. List of Co-based FT Catalysts Formulated (Contd.)

\begin{tabular}{|c|c|c|c|c|c|c|}
\hline बAग & \%wt\% G o & $\% \%$ \%tom & \%o\% proun & \%owpoort & 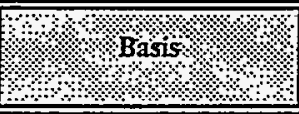 & prepar \\
\hline Co.017 & $20 \%$ & $0.5 \% R u$ & $1 \% \mathrm{La}_{2} \mathrm{O}_{3}$ & $\gamma$-Alumina & $\begin{array}{l}\text { Similar to Co.003, } \\
\text { but all aqueous }\end{array}$ & P \\
\hline Co.018 & $20 \%$ & $0.5 \%$ Ru & 0 & $\gamma$-Alumina & $\begin{array}{l}\text { Ru-Promoted Catalyst } \\
\text { (single-step, aqueous } \\
\text { IW) }\end{array}$ & $P$ \\
\hline Co.019 & $20 \%$ & $0.5 \% \mathrm{Ru}$ & 0 & Silica & $\begin{array}{c}\text { Ru-Promoted Catalyst } \\
\text { (single-step, aqueous } \\
\text { IW) }\end{array}$ & $p$ \\
\hline Co.020 & 0 & $0.5 \% \mathrm{Ru}$ & 0 & $\gamma$-Alumina & Ru Base Catalyst & $\mathbf{P}$ \\
\hline $\mathrm{C} 0.020 \mathrm{~A}$ & $20 \%$ & $0.4 \% \mathrm{Ru}$ & 0 & $\gamma$-Alumina & $\begin{array}{l}\text { Co added to dried } \\
\text { Co.020 by IW } \\
\text { impreg. }\end{array}$ & $\mathbf{P}$ \\
\hline $\mathrm{C} 0.020 \mathrm{~B}$ & $20 \%$ & $0.4 \% \mathrm{Ru}$ & 0 & $\gamma$-Alumina & $\begin{array}{l}\text { Co added to reduced } \\
\text { Co.020 by IW } \\
\text { impreg. }\end{array}$ & $\mathbf{P}$ \\
\hline Co.020C & $20 \%$ & $0.4 \% \mathrm{Ru}$ & 0 & $\gamma$-Alumina & $\begin{array}{l}\text { Co added to calcined } \\
\text { Co.020 by IW } \\
\text { impreg. }\end{array}$ & P \\
\hline Co.021 & $20 \%$ & 0 & $0.7 \% \mathrm{Zr}$ & Silica & $\begin{array}{l}\text { U.K. Pat Appl. } \\
\text { GB } 2125062 \text { A } \\
\text { (single-step) }\end{array}$ & $\mathbf{P}$ \\
\hline Co.022 & $20 \%$ & 0 & $0.7 \% \mathrm{Zr}$ & Silica & $\begin{array}{l}\text { U.K. Pat. Appl. } \\
\text { GB } 2125062 \text { A } \\
\text { (multiple-steps) }\end{array}$ & $\mathbf{P}$ \\
\hline Co.023 & $20 \%$ & 0 & $1.4 \% \mathrm{Zr}$ & Silica & $\begin{array}{l}\text { U.K. Pat Appl. } \\
\text { GB } 2125062 \text { A } \\
\text { (multiple-steps) }\end{array}$ & $\mathbf{p}$ \\
\hline Co.024 & $20 \%$ & 0 & $\begin{array}{c}8.5 \% \mathrm{Zr} \\
.\end{array}$ & Silica & $\begin{array}{c}\text { Eur. Pat. Appl. } \\
0167215 \mathrm{A2} \\
\text { (non-aqueous } \mathrm{Zr} \text { pre- } \\
\text { impregnation) }\end{array}$ & $\mathbf{P}$ \\
\hline Co.025 & $20 \%$ & 0 & $8.5 \% \mathrm{Zr}$ & Silica & $\begin{array}{l}\text { Similar to } \mathrm{Co.024} \text { but } \\
\text { aqueous } \mathrm{Zr} \text { pre- } \\
\text { impregnation }\end{array}$ & $\mathbf{P}$ \\
\hline Co.026 & $20 \%$ & 0 & 0 & $\begin{array}{c}\text { Silica } \\
\text { (Davison Grade } \\
59 \text { ) }\end{array}$ & $\begin{array}{c}\text { Similar to Co.012 } \\
\text { but different grade } \\
\text { silica }\end{array}$ & $\mathbf{P}$ \\
\hline$C 0.027$ & $20 \%$ & $0.5 \% \mathrm{Ru}$ & 0 & $\begin{array}{c}\text { Silica } \\
\text { (Davison Grade } \\
59 \text { ) } \\
\end{array}$ & $\begin{array}{c}\text { Similar to Co.019 } \\
\text { but different grade } \\
\text { silica }\end{array}$ & P \\
\hline Co.028 & $20 \%$ & $0.5 \% \mathrm{Ru}$ & $0.5 \% \mathrm{~K}$ & $\gamma$-alumina & $\begin{array}{c}\text { Ru- and K-Promoted } \\
\text { Catalyst (single-step } \\
\text { IW) }\end{array}$ & $\mathbf{P}$ \\
\hline Co.029 & $30 \%$ & $0.5 \% \mathrm{Ru}$ & $0.5 \% \mathrm{~K}$ & $\gamma$-alumina & $\begin{array}{c}\text { Similar to Co.028 but } \\
30 \% \text { Co }\end{array}$ & $\mathbf{P}$ \\
\hline
\end{tabular}


Table 1. List of Co-based FT Catalysts Formulated (Contd.)

\begin{tabular}{|c|c|c|c|c|c|c|}
\hline CAT. No, & \% \% $\mathrm{C}_{0} \%$ & \% & W\%topromp & Support & W. & Prepar \\
\hline Co.030 & $20 \%$ & 0 & $0.1 \% \mathrm{Zr}$ & $\gamma$-alumina & $\begin{array}{c}\text { Zr-Promoted Catalyst } \\
\text { (single-step aqueous } \\
\text { IW) }\end{array}$ & $\mathbf{P}$ \\
\hline$C_{0.031}$ & $20 \%$ & 0 & $1.4 \% \mathrm{Zr}$ & $\gamma$-alumina & $\begin{array}{l}\text { Zr-Promoted } \\
\text { Catalyst (single-step } \\
\text { aqueous IW) }\end{array}$ & $\mathbf{P}$ \\
\hline Co.032 & $20 \%$ & 0 & $8.5 \% \mathrm{Zr}$ & $\gamma$-alumina & $\begin{array}{l}\text { Zr-Promoted } \\
\text { Catalyst (aqueous IW } \\
\text { co-impregnation) }\end{array}$ & $\mathbf{P}$ \\
\hline Co.033 & $20 \%$ & 0 & $8.5 \% \mathrm{Zr}$ & $\gamma$-alumina & $\begin{array}{l}\text { multiple-steps, } \\
\text { aqueous IW, Co pre- } \\
\text { impregnation }\end{array}$ & $\mathbf{P}$ \\
\hline Co.034 & $20 \%$ & 0 & $8.5 \% \mathrm{Zr}$ & $\gamma$-alumina & $\begin{array}{l}\text { multiple-steps, } \\
\text { aqueous IW, } \mathrm{Zr} \text { pre- } \\
\text { impregnation }\end{array}$ & $\mathbf{P}$ \\
\hline Co.035 & $20 \%$ & 0 & $8.5 \% \mathrm{Zr}$ & Silica & $\begin{array}{l}\text { Zr-Promoted } \\
\text { Catalyst (single-step } \\
\text { aqueous, kneaded) }\end{array}$ & $\mathbf{P}$ \\
\hline Co.036 & $20 \%$ & 0 & $8.5 \% \mathrm{Zr}$ & Silica & $\begin{array}{l}\text { multiple-steps, aq. Co } \\
\text { pre-impreg. by } \\
\text { kneading, aqueous } \\
\text { IW Zr impreg. }\end{array}$ & $\mathbf{P}$ \\
\hline Co.037 & $12 \%$ & 0 & 0 & Titania & $\begin{array}{c}\text { Similar to } \mathrm{Co.} 013, \\
\text { but } \mathrm{TiO}_{2} \text { mainly } \\
\text { anatase }\end{array}$ & $\mathbf{P}$ \\
\hline Co.038 & $12 \%$ & $0.5 \% \mathrm{Ru}$ & 0 & Titania & $\begin{array}{c}\text { Similar to } \mathrm{Co.014}, \\
\text { but } \mathrm{TiO}_{2} \text { mainly } \\
\text { anatase }\end{array}$ & $\mathbf{P}$ \\
\hline Co.039 & $12 \%$ & 0 & 0 & Titania & $\begin{array}{l}\text { Similar to Co.007, } \\
\text { but only } 12 \% \mathrm{Co}\end{array}$ & $\mathbf{P}$ \\
\hline Co.040 & $12 \%$ & 0 & 0 & Titania & $\begin{array}{c}\text { Similar to Co.013, } \\
\text { but only } 12 \% \text { Co }\end{array}$ & $\mathbf{P}$ \\
\hline Co.041 & $20 \%$ & $0.5 \% \mathrm{Ru}$ & $8.5 \% \mathrm{Zr}$ & Silica & $\begin{array}{l}\text { Similar to Co.025, } \\
\text { but Ru-promoted }\end{array}$ & $\mathbf{P}$ \\
\hline Co.042 & 0 & 0 & $8.5 \% \mathrm{Zr}$ & Silica & $\begin{array}{l}\text { Blank } \mathrm{Zr} \text {-promoted } \\
\qquad \mathrm{SiO}_{2}\end{array}$ & $\mathbf{P}$ \\
\hline Co.043 & $20 \%$ & $0.5 \% \mathrm{Ru}$ & $\begin{array}{l}8.5 \% \mathrm{Zr} \\
0.5 \% \mathrm{~K}\end{array}$ & Silica & $\begin{array}{c}\text { Similar to Co.041, } \\
\text { but K-promoted }\end{array}$ & $\mathbf{P}$ \\
\hline Co.044 & $20 \%$ & 0 & $15 \% \mathrm{Zr}$ & Silica & $\begin{array}{l}\text { Similar to Co.025, } \\
\text { but } 15 \% \mathrm{Zr}\end{array}$ & $\mathbf{P}$ \\
\hline Co.045 & $20 \%$ & 0 & $4 \% \mathrm{Zr}$ & Silica & $\begin{array}{c}\text { Similar to Co.025, } \\
\text { but } 4 \% \mathrm{Zr}\end{array}$ & $\mathbf{P}$ \\
\hline Co.046 & $12 \%$ & $0.75 \% \operatorname{Re}$ & $4 \% \mathrm{SiO}_{2}$ & Titania & $\begin{array}{l}\text { Similar to } \mathrm{Co.006} \text {, } \\
\text { but } \mathrm{SiO}_{2} \text {-promoted }\end{array}$ & - \\
\hline
\end{tabular}


Table 1. List of Co-based FT Catalysts Formulated (Contd.)

\begin{tabular}{|c|c|c|c|c|c|c|}
\hline CATNOे। & \%w\% Ga & \%ै⿴囗十) & \%ri\% Prom, & \% support & W. & Prepars \\
\hline Co.047 & $20 \%$ & $0.5 \% \mathrm{Ru}$ & $0.3 \% \mathrm{~K}$ & $\gamma$-alumina & $\begin{array}{l}\text { Similar to Co.028, } \\
\text { but } 0.3 \% \mathrm{~K}\end{array}$ & $\mathbf{P}$ \\
\hline Co.048 & $20 \%$ & $0.5 \% \mathrm{Ru}$ & $\begin{array}{l}8.5 \% \mathrm{Zr} \\
0.3 \% \mathrm{~K}\end{array}$ & Silica & $\begin{array}{l}\text { Similar to Co.043, } \\
\text { but } 0.3 \% \mathrm{~K}\end{array}$ & $\mathbf{P}$ \\
\hline Co.049 & $20 \%$ & $0.5 \% \mathrm{Ru}$ & $0.1 \% \mathrm{~K}$ & $\gamma$-alumina & $\begin{array}{c}\text { Similar to Co.047, } \\
\text { but } 0.1 \% \mathrm{~K}\end{array}$ & $\mathbf{P}$ \\
\hline Co.050 & $20 \%$ & $0.5 \% \mathrm{Ru}$ & $\begin{array}{l}8.5 \% \mathrm{Zr} \\
0.1 \% \mathrm{~K} \\
\end{array}$ & Silica & $\begin{array}{c}\text { Similar to Co.048, } \\
\text { but } 0.1 \% \mathrm{~K}\end{array}$ & $\mathbf{P}$ \\
\hline Co.051 & 0 & $0.5 \% \mathrm{Ru}$ & 0 & Titania (R) & & $\mathbf{P}$ \\
\hline Co.052 & 0 & $0.5 \% \mathrm{Ru}$ & 0 & Titania (A) & & $\mathbf{P}$ \\
\hline Co.053 & $20 \%$ & $0.5 \% \mathrm{Ru}$ & 0 & $\gamma$-alumina & $\begin{array}{l}\text { Similar to Co. } 018 \\
\text { (New Batch) }\end{array}$ & $\mathbf{P}$ \\
\hline Co.054 & $20 \%$ & 0 & $\begin{array}{l}8.5 \% \mathrm{Zr} \\
0.3 \% \mathrm{~K}\end{array}$ & Silica & $\begin{array}{l}\text { Similar to Co.043, } \\
\text { but without } \mathrm{Ru}\end{array}$ & $\mathbf{P}$ \\
\hline Co.055 & $20 \%$ & $1 \% \operatorname{Re}$ & $1 \% \mathrm{La}_{2} \mathrm{O}_{3}$ & $\gamma$-alumina & $\begin{array}{l}\text { Similar to Co.001, } \\
\text { but without K }\end{array}$ & $\mathbf{P}$ \\
\hline Co.056 & $20 \%$ & 0 & $8.5 \% \mathrm{La}_{2} \mathrm{O}_{3}$ & Silica & $\begin{array}{l}\text { Similar to Co.025, } \\
\text { but La instead of } \mathrm{Zr}\end{array}$ & $\mathbf{P}$ \\
\hline Co $057 \%$ & $20 \%$ & $1 \% \operatorname{Re}$ & $1 \% \mathrm{La}_{2} \mathrm{O}_{3}$ & Silica & $\begin{array}{c}\text { Similar to Co.055, } \\
\text { but with } \mathrm{SiO}_{2} \text { support }\end{array}$ & $\mathbf{P}$ \\
\hline Co.058, & $20 \%$ & 0 & $1 \% \mathrm{La}_{2} \mathrm{O}_{3}$ & $\gamma$-alumina & $\begin{array}{c}\text { Similar to Co.055, } \\
\text { but without Re (one- } \\
\text { step impreg.) }\end{array}$ & $\mathbf{P}$ \\
\hline $\mathrm{Co} .059 \% \%$ & $20 \%$ & 0 & $1 \% \mathrm{La}_{2} \mathrm{O}_{3}$ & $\gamma$-alumina & $\begin{array}{c}\text { Similar to Co.015, } \\
\text { but without Re } \\
\text { (two-step impreg.) }\end{array}$ & $\mathbf{P}$ \\
\hline Co. $060 \%$ & $30 \%$ & 0 & 0 & $\gamma$-alumina & $\begin{array}{c}\text { Similar to Co.005, } \\
\text { but with } 30 \% \text { Co }\end{array}$ & $\mathbf{P}$ \\
\hline Co.061 \% \% & $30 \%$ & $0.5 \% \mathrm{Ru}$ & 0 & $\gamma$-alumina & $\begin{array}{c}\text { Similar to Co.053, } \\
\text { but with } 30 \% \mathrm{Co}\end{array}$ & $\mathbf{P}$ \\
\hline Co.062\% & $30 \%$ & $0.5 \% \mathrm{Ru}$ & $1.5 \% \mathrm{~K}$ & $\boldsymbol{\gamma}$-alumina & $\begin{array}{c}\text { Similar to Co.029, } \\
\text { but with } 1.5 \% \mathrm{~K}\end{array}$ & $\mathbf{P}$ \\
\hline Co $063 \%$ & $20 \%$ & 0 & $8.5 \% \mathrm{La}$ & $\gamma$-alumina & $\begin{array}{c}\text { Two-steps, aqueous } \\
\text { IW, La pre- } \\
\text { impregnation } \\
\end{array}$ & $\mathbf{P}$ \\
\hline $\begin{array}{l}\text { CAL.01 } \\
(97 \mathrm{E}-13 \mathrm{E}) \\
\end{array}$ & $20 \%$ & $0.5 \% \mathrm{Ru}$ & 0 & $\gamma$-alumina & $\begin{array}{c}\text { Similar to Co.018 } \\
\text { (Calsicat Prep.) }\end{array}$ & $\mathbf{P}$ \\
\hline $\begin{array}{l}\text { CAL.02 } \\
\quad(97 \mathrm{E}-16 \mathrm{~B}) \\
\end{array}$ & $20 \%$ & $0.5 \% \mathrm{Ru}$ & 0 & $\gamma$-alumina & $\begin{array}{c}\text { Similar to CAL.01 } \\
\text { (New Batch) }\end{array}$ & $\mathbf{P}$ \\
\hline $\begin{array}{l}\text { CAL.03 } \\
\quad \text { (97E-13F }\end{array}$ & $20 \%$ & $0.5 \% \mathrm{Ru}$ & 0 & $\gamma$-alumina & $\begin{array}{l}\text { 2-step impreg., } \\
\text { Simlar to CAL.02 }\end{array}$ & $\mathbf{P}$ \\
\hline
\end{tabular}


Table 1. List of Co-based FT Catalysts Formulated (Contd.)

\begin{tabular}{|c|c|c|c|c|c|c|}
\hline \%A & +wt\%, co & \% & He\%prom & SYuport & & prepar \\
\hline $\begin{array}{l}\text { CAL.04 } \\
\text { (97E-50C) }\end{array}$ & $20 \%$ & $0.5 \% \mathrm{Ru}$ & $0.3 \% \mathrm{~K}$ & $\gamma$-alumina & $\begin{array}{l}\text { Similar to Co.047 } \\
\text { Stand. Calc. in Air }\end{array}$ & $\mathbf{p}$ \\
\hline $\begin{array}{l}\text { CAL.05 } \\
\text { (97E-50D) }\end{array}$ & $20 \%$ & $0.5 \% \mathrm{Ru}$ & $0.3 \% \mathrm{~K}$ & $\gamma$-alumina & $\begin{array}{l}\text { Similar to Co.047 } \\
\text { Stand. Calc. in } \mathrm{N}_{2}\end{array}$ & $\mathbf{P}$ \\
\hline $\begin{array}{l}\text { CAL.06 } \\
\text { (97E-51A) }\end{array}$ & $20 \%$ & $0.5 \% \mathrm{Ru}$ & $0.3 \% \mathrm{~K}$ & $\gamma$-alumina & $\begin{array}{l}\text { Similar to Co.047 } \\
\text { Red. and Pas. in Air }\end{array}$ & $\mathbf{P}$ \\
\hline $\begin{array}{l}\text { CAL.07 } \\
\text { (97E-51B) }\end{array}$ & $20 \%$ & $0.5 \% \mathrm{Ru}$ & $0.3 \% \mathrm{~K}$ & $\gamma$-alumina & $\begin{array}{l}\text { Similar to Co.047 } \\
\text { Red. and Protected in } \\
\text { Paraffin ( } 51.3 \% \text { Cat. } \\
\text { Conc.) }\end{array}$ & $\mathbf{P}$ \\
\hline $\begin{array}{l}\text { CAL.08 } \\
\text { (97E-51C) }\end{array}$ & $20 \%$ & $0.5 \% \mathrm{Ru}$ & $0.3 \% \mathrm{~K}$ & $\gamma$-alumina & $\begin{array}{l}\text { Similar to Co. } 047 \\
\text { Calc., Red. and } \\
\text { Protected in Paraffin } \\
\text { (54.4\% Cat. Conc.) }\end{array}$ & $\mathbf{P}$ \\
\hline calog $\%$ \% & $20 \%$ & $0.5 \% \mathrm{Ru}$ & $0.3 \% \mathrm{~K}$ & $\gamma$-alumina & $\begin{array}{l}\text { Similar to CAL.0S, } \\
\text { Reduced and } \\
\text { Protected in Soya } \\
\text { (51.7\% Cat. Conc.) }\end{array}$ & $P$ \\
\hline \%Al $10 \%$ \% & $20 \%$ & $0.5 \% \mathrm{Ru}$ & $0.3 \% \mathrm{~K}$ & $\begin{array}{l}\gamma \text {-alumina } \\
\text { (Condea) }\end{array}$ & $\begin{array}{l}\text { Similar to CAL.05, } \\
\text { but on Condea } \mathrm{Al}_{2} \mathrm{O}_{3}\end{array}$ & $\mathbf{P}$ \\
\hline
\end{tabular}


Table 2. List of Water-Gas Shift Catalysts and F-T Catalysts with WGS Function

\begin{tabular}{|c|c|c|c|c|c|c|}
\hline 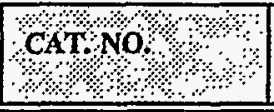 & काt $\mathrm{Co}^{\circ}$ & \% wr\%m & \%homp prom & W. Support & W Basis & Prepar \\
\hline WGS.01 & 0 & $5.0 \% \mathrm{Cu}$ & 0 & $\gamma$-alumina & single-step, aqu. IW & $\mathbf{P}$ \\
\hline WGS.02 & 0 & $5.0 \% \mathrm{Cu}$ & $10 \% \mathrm{Zn}$ & $\gamma$-alumina & single-step, aqu. IW & $\mathbf{P}$ \\
\hline WGS.03 & 0 & $5.0 \% \mathrm{Cu}$ & $4.0 \% \mathrm{Cr}$ & $\gamma$-alumina & single-step, aqu. IW & $\mathbf{P}$ \\
\hline WGS.04 & 0 & 0 & $4.0 \% \mathrm{Cr}$ & $\gamma$-alumina & single-step, aqu. IW & $\mathbf{P}$ \\
\hline WGS.05 & 0 & 0 & $4.0 \% \mathrm{Mn}$ & $\gamma$-alumina & single-step, aqu. IW & $\mathbf{P}$ \\
\hline WGS.06 & 0 & $5.0 \% \mathrm{Cu}$ & $4.0 \% \mathrm{Mn}$ & $\gamma$-alumina & single-step, aqu. IW & $\mathbf{P}$ \\
\hline WGS.07 & 0 & $4.0 \% \mathrm{Fe}$ & 0 & $\gamma$-alumina & single-step, aqu. IW & $\mathbf{P}$ \\
\hline WGS.08 & 0 & $4.0 \% \mathrm{Fe}$ & $4.0 \% \mathrm{Cr}$ & $\gamma$-alumina & single-step, aqu. IW & $\mathbf{P}$ \\
\hline WGS.09 & 0 & $5.0 \% \mathrm{Cu}$ & $4.0 \% \mathrm{Cr}$ & $\gamma$-alumina & Similar to WGS.03 & $\mathbf{P}$ \\
\hline CoW.01 & $20 \%$ & $5.0 \% \mathrm{Cu}$ & $10 \% \mathrm{Zn}$ & $\gamma$-alumina & $\begin{array}{l}\text { multiple-steps, aqu. } \\
\text { IW, Cu, } \mathrm{Zn} \text { pre-impr. }\end{array}$ & $\mathbf{P}$ \\
\hline CoW.02 & $20 \%$ & $5.0 \% \mathrm{Cu}$ & $4.0 \% \mathrm{Cr}$ & $\gamma$-alumina & $\begin{array}{l}\text { multiple-steps, aqu. } \\
\text { IW, Cu,Cr pre-impr. }\end{array}$ & $\mathbf{P}$ \\
\hline CoW.03 & $10 \%$ & $5.0 \% \mathrm{Cu}$ & $4.0 \% \mathrm{Cr}$ & $\gamma$-alumina & $\begin{array}{c}\text { similar to CoW.02 } \\
\text { (10\% Co only) }\end{array}$ & $\mathbf{P}$ \\
\hline CoW.04 & $10 \%$ & $10 \% \mathrm{Cu}$ & $8.0 \% \mathrm{Cr}$ & $\gamma$-alumina & $\begin{array}{l}\text { similar to CoW.03; } \\
10 \% \mathrm{Cu} \text { and } 8 \% \mathrm{Cr}\end{array}$ & $\mathbf{P}$ \\
\hline Cow.05 & $20 \%$ & $5.0 \% \mathrm{Cu}$ & $4.0 \% \mathrm{Cr}$ & $\gamma$-alumina & $\begin{array}{c}\text { similar to CoW.02 } \\
\text { with support calcined } \\
\text { at } 750^{\circ} \mathrm{C} \\
\end{array}$ & $\mathbf{P}$ \\
\hline Cow.06 & $20 \%$ & $5.0 \% \mathrm{Cu}$ & $4.0 \% \mathrm{Cr}$ & Silica & $\begin{array}{l}\text { multiple-steps, aqu. } \\
\text { IW, Cu,Cr pre-impr. }\end{array}$ & $\mathbf{P}$ \\
\hline CoW.07 & $20 \%$ & $5.0 \% \mathrm{Cu}$ & $4.0 \% \mathrm{Cr}$ & Silica & $\begin{array}{l}\text { similar to CoW.06, } \\
\text { but with support } \\
\text { calcined at } 750^{\circ} \mathrm{C} \\
\end{array}$ & $\mathbf{P}$ \\
\hline Cow & $30 \%$ & $5.0 \% \mathrm{Cu}$ & $4.0 \% \mathrm{Cr}$ & $\gamma$-alumina & $\begin{array}{c}\text { Similar to CoW.05, } \\
\text { but with } 30 \% \text { Co }\end{array}$ & $\mathbf{P}$ \\
\hline Cowog\% & $20 \%$ & $10.0 \% \mathrm{Cu}$ & $8.0 \% \mathrm{Cr}$ & Silica & $\begin{array}{c}\text { similar to CoW.06, } \\
\text { but with } 10 \% \mathrm{Cu} \text { and } \\
8 \% \mathrm{Cr}\end{array}$ & $\mathbf{P}$ \\
\hline Co & $\begin{array}{c}10 \% \mathrm{Co}, 10 \% \\
\mathrm{Fe} \\
\end{array}$ & $0.5 \% \mathrm{Ru}$ & 0 & $\gamma$-alumina & Single Step, IW & $\mathbf{P}$ \\
\hline cow & $\begin{array}{c}10 \% \text { Co, } 10 \% \\
\mathrm{Fe} \\
\end{array}$ & $0.5 \% \mathrm{Ru}$ & $0.5 \% \mathrm{~K}$ & $\gamma$-alumina & Single Step, IW & $\mathbf{P}$ \\
\hline cow $12 \%$ & $\begin{array}{c}10 \% \mathrm{Co}, 10 \% \\
\mathrm{Fe}\end{array}$ & $0.5 \% \mathrm{Ru}$ & 0 & Silica & Single Step, IW & $\mathbf{P}$ \\
\hline
\end{tabular}


Table 3. Summary of Physical Properties

\begin{tabular}{|c|c|c|c|c|c|}
\hline CATAYYST & औ' Composition & $\begin{array}{l}\text { BET Surface } \\
\text { Area (m/g) } \\
\text { (m) }\end{array}$ & $\begin{array}{l}\text { Pore } \\
\text { volume } \\
\text { focfor) }\end{array}$ & $\begin{array}{l}\text { Average } \\
\text { pore bia. } \\
\text { (A) }\end{array}$ & $\begin{array}{l}\text { Average } \\
\text { Part. Size } \\
(\mu \mathrm{mi}) \text { ) }\end{array}$ \\
\hline Vista-B $\mathrm{Al}_{2} \mathrm{O}_{3}$ & (0-400 mesh) & 299 & 1.69 & 269 & 24 \\
\hline Vista- $\mathrm{B} \mathrm{Al}_{2} \mathrm{O}_{3}$ & Calcined $500^{\circ} \mathrm{C}$ & 240 & 0.49 & 82 & \\
\hline Vista-B $\mathrm{Al}_{2} \mathrm{O}_{3}$ & Calcined $600^{\circ} \mathrm{C}$ & 206 & 0.49 & 95 & \\
\hline Vista- $\mathrm{B} \mathrm{Al}_{2} \mathrm{O}_{3}$ & Calcined $600^{\circ} \mathrm{C}$ & 174 & 0.47 & 109 & \\
\hline Condea $\mathrm{Al}_{2} \mathrm{O}_{3}$ & (as received) & 219 & 0.53 & 97 & 77 \\
\hline Davison $\mathrm{SiO}_{2}$ & (0-400 mesh) & 219 & 0.51 & 89 & 121 \\
\hline Degussa $\mathrm{P}_{25} \mathrm{TiO}_{2}$ & (as received) & 47 & 0.40 & 335 & 6 \\
\hline Degussa $\mathrm{P} 25 \mathrm{TiO}_{2}$ & $\begin{array}{c}\text { Dried } 60^{\circ} \mathrm{C} \text {, Calc. } \\
350^{\circ} \mathrm{C} / 16 \mathrm{hrs} \\
\end{array}$ & 46 & 0.48 & 419 & 51 \\
\hline Degussa P25 $\mathrm{TiO}_{2}$ & $\begin{array}{c}\text { Dried } 60^{\circ} \mathrm{C} \text {, Calc. } \\
650^{\circ} \mathrm{C} / 16 \mathrm{hrs}\end{array}$ & 12 & 0.17 & 573 & 212 \\
\hline Co.001 & $\begin{array}{c}20 \mathrm{Co} / 1 \mathrm{Re} / \\
1 \mathrm{La}_{2} \mathrm{O}_{3} / 0.13 \mathrm{~K} / \mathrm{A}\end{array}$ & 191 & 0.31 & 66 & $\begin{array}{l}42 \\
40 \\
\end{array}$ \\
\hline Co.002 & $\begin{array}{c}20 \mathrm{Co} / 0.43 \mathrm{Ru} / \\
1 \mathrm{La}_{2} \mathrm{O}_{3} / \mathrm{A}\end{array}$ & 149 & 0.33 & 89 & 74 \\
\hline Co.003 & $\begin{array}{c}20 \mathrm{Co} / 0.5 \mathrm{Ru} / \\
1 \mathrm{La}_{2} \mathrm{O}_{3} / \mathrm{A}\end{array}$ & 122 & 0.26 & 87 & 77 \\
\hline Co.004 & $\begin{array}{c}20 \mathrm{Co} / 0.43 \mathrm{Ru} / \\
1 \mathrm{La}_{2} \mathrm{O}_{3} / \mathrm{A} \\
\end{array}$ & & & & 78 \\
\hline Co.005 & $20 \mathrm{Co} / \mathrm{A}$ & 173 & 0.34 & 81 & $\begin{array}{l}65 \\
86 \\
\end{array}$ \\
\hline Co.006 & $12 \mathrm{Co} / 0.75 \mathrm{Re} / \mathrm{T}$ & 16 & - & - & 121 \\
\hline Co.007 & $20 \mathrm{Co} / \mathrm{T}$ & 11 & 0.10 & 372 & \\
\hline Co.008 & $20 \mathrm{Co} / \mathrm{S}$ & 181 & 1.06 & 234 & \\
\hline Co.009 & $20 \mathrm{Co} / 0.5 / \mathrm{A}$ & & & & \\
\hline Co. 010 & $20 \mathrm{Co} / \mathrm{A}$ & & & & \\
\hline Co.011 & $20 \mathrm{Co} / \mathrm{S}$ & 211 & 1.07 & 203 & 107 \\
\hline Co.012 & $20 \mathrm{Co} / \mathrm{S}$ & 211 & - & - & 105 \\
\hline Co. 013 & $20 \mathrm{Co} / \mathrm{T}$ & & & & \\
\hline Co.014 & $12 \mathrm{Co} / 0.5 \mathrm{Ru} / \mathrm{T}$ & 15 & 0.12 & 328 & 111 \\
\hline
\end{tabular}


Table 3. Summary of Physical Properties (contd.)

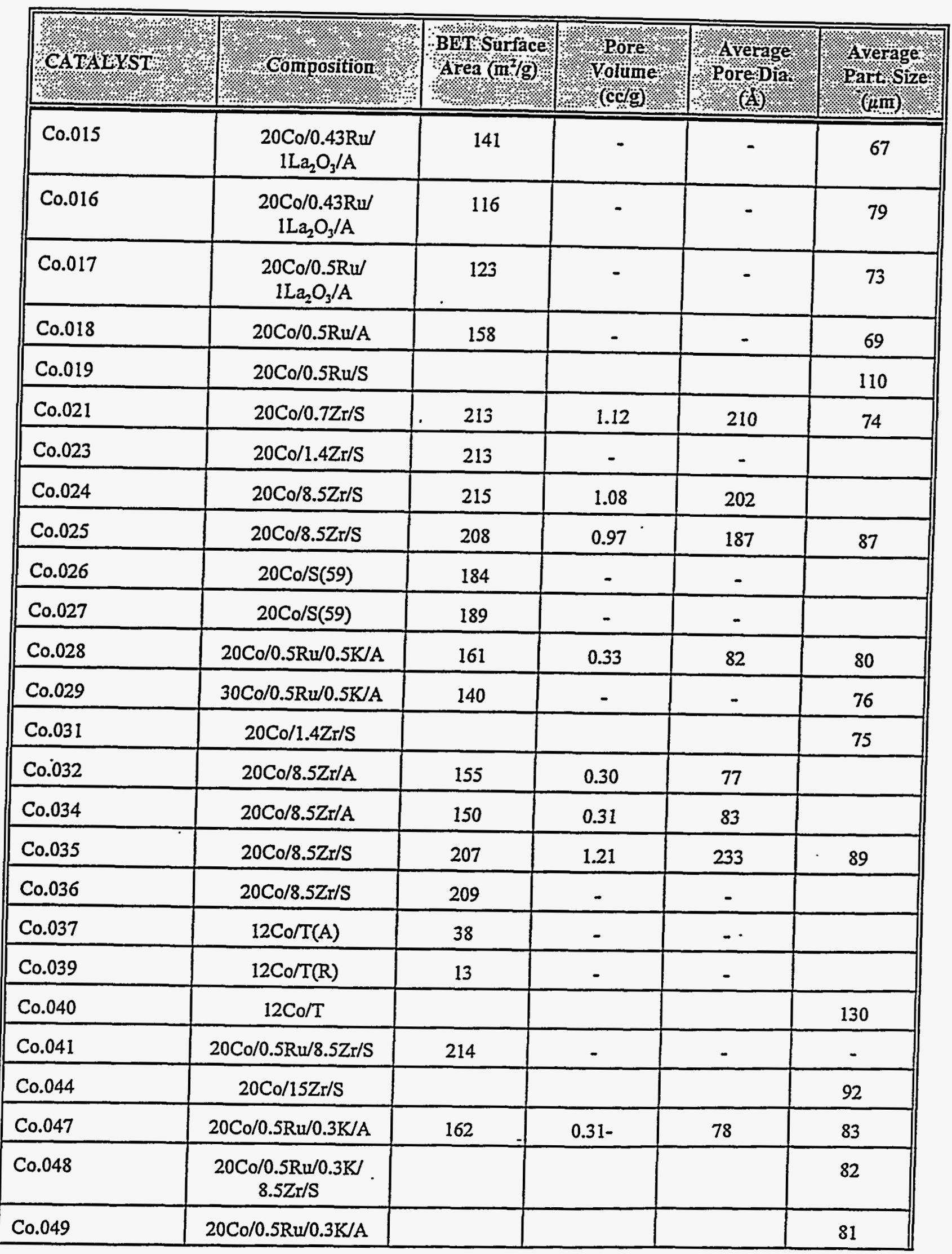


Table 3. Summary of Physical Properties (contd.)

\begin{tabular}{|c|c|c|c|c|c|}
\hline CATAYYST & ४ै Composition & BET Surface & $\begin{array}{l}\text { Polume } \\
\text { vore } \\
.(\operatorname{cctg})\end{array}$ & $\begin{array}{l}\text { arerage } \\
\text { porepia } \\
\text { ( }(\mathrm{A})\end{array}$ & $\begin{array}{l}\text { Average: } \\
\text { Part Size } \\
\text { (unt) }\end{array}$ \\
\hline Co.053 & $20 \mathrm{Co} / 0.5 \mathrm{Ru} / \mathrm{A}$ & & & & 75 \\
\hline Co.054 & $20 \mathrm{Co} / 0.3 \mathrm{~K} / 8.5 \mathrm{Zr} / \mathrm{S}$ & & & & 101 \\
\hline Co.055 & $\begin{array}{c}20 \mathrm{Co} / 1 \mathrm{Re} / \\
1 \mathrm{La}_{2} \mathrm{O}_{3} / \mathrm{A}\end{array}$ & & & & 86 \\
\hline Co.056 & $20 \mathrm{Co} / 8.5 \mathrm{La}_{2} \mathrm{O}_{3} / \mathrm{S}$ & & & & 100 \\
\hline CAL.01 & $20 \mathrm{Co} / 0.5 \mathrm{Ru} / \mathrm{A}$ & 178 & 0.34 & 76 & \\
\hline CAL.02 & $20 \mathrm{Co} / 0.5 \mathrm{Ru} / \mathrm{A}$ & 158 & - & - & 74 \\
\hline CAL.03 & $20 \mathrm{Co} / 0.5 \mathrm{Ru} / \mathrm{A}$ & 158 & - & - & \\
\hline CAL.04 & $20 \mathrm{Co} / 0.5 \mathrm{Ru} / 0.3 \mathrm{~K} / \mathrm{A}$ & 151 & & & 72 \\
\hline CAL.05 & $20 \mathrm{Co} / 0.5 \mathrm{Ru} / 0.3 \mathrm{~K} / \mathrm{A}$ & 163 & & & 73 \\
\hline CAL.06 & $20 \mathrm{Co} / 0.5 \mathrm{Ru} / 0.3 \mathrm{~K} / \mathrm{A}$ & 162 & & & 72 \\
\hline CAL.07 & $20 \mathrm{Co} / 0.5 \mathrm{Ru} / 0.3 \mathrm{~K} / \mathrm{A}$ & - & & & \\
\hline CAL.08 & $20 \mathrm{Co} / 0.5 \mathrm{Ru} / 0.3 \mathrm{~K} / \mathrm{A}$ & - & & & 63 \\
\hline CAL.09 & $20 \mathrm{Co} / 0.5 \mathrm{Ru} / 0.3 \mathrm{~K} / \mathrm{A}$ & & & & 63 \\
\hline CAL.10 & $20 \mathrm{Co} / 0.5 \mathrm{Ru} / 0.3 \mathrm{~K} / \mathrm{A}$ & 142 & - & - & 83 \\
\hline
\end{tabular}


Table 4. $\mathrm{H}_{2}$ Chemisorption and TPR Results

\begin{tabular}{|c|c|c|c|c|c|c|}
\hline \multirow{2}{*}{ 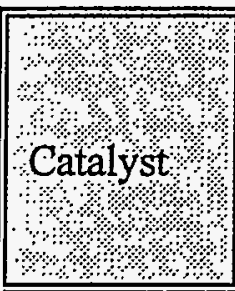 } & \multicolumn{6}{|c|}{ 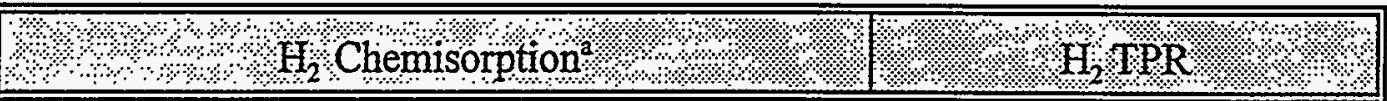 } \\
\hline & $\begin{array}{l}\text { Total } \\
\left(\mu \mathrm{mol}^{2}\right. \\
\left.\mathrm{H}_{2} / \mathrm{g} \mathrm{cat}\right)\end{array}$ & $\begin{array}{l}\text { Irrevers } \\
\text { (ji mol } \\
\text { H } 1 \mathrm{~g} \mathrm{cat})\end{array}$ & $\begin{array}{l}\text { Average } \\
d^{6}{ }^{2} \\
(n m)_{1}\end{array}$ & Disp. & 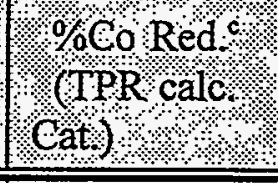 & $\begin{array}{l}0 \% \text { Red) } \\
\text { (stand. } \\
\text { red) }\end{array}$ \\
\hline Co.001 & $174 \pm 5$ & $157 \pm 5$ & & 10.2 & & \\
\hline Co. 002 & 155 & 130 & & 9.1 & & \\
\hline Co.003 & 165 & 140 & & 9.6 & & \\
\hline $\begin{array}{r}\text { Co.004 }{ }^{\mathrm{f}}(1) \\
(2) \\
(3) \\
(4)\end{array}$ & $\begin{array}{l}205 \\
132 \\
144 \\
136 \\
\end{array}$ & $\begin{array}{l}185 \\
116 \\
123 \\
122 \\
\end{array}$ & $\begin{array}{l}7 \\
11 \\
10 \\
10\end{array}$ & $\begin{array}{l}12.1 \\
6.6 \\
7.2 \\
6.8 \\
\end{array}$ & $\begin{array}{l}91 \\
55 \\
46 \\
41 \\
\end{array}$ & \\
\hline Co.005 & 48 & 42 & 20 & 2.8 & 89 & 58 \\
\hline Co.005a & 129 & 110 & 11 & 7.6 & 92 & 84 \\
\hline Co.006 & 44 & 32 & 19 & 4 & 80 & \\
\hline Co.007 & 33 & 23 & 36 & 2.0 & 78 & 78 \\
\hline \multicolumn{7}{|l|}{ Co.008 } \\
\hline Co.009 & 133 & 109 & 13 & 7.8 & 98 & \\
\hline \multicolumn{7}{|l|}{ Co. 010} \\
\hline Co.010a & 124 & 100 & 12.5 & 7.3 & 91 & 89 \\
\hline Co.011 & 82 & 70 & 15.6 & 4.8 & 75 & 75 \\
\hline Co.012 & 89 & 74 & 15 & 5.2 & 80 & 80 \\
\hline Co.013 & 21 & 17 & 79 & 1.2 & 97 & \\
\hline Co.014 & 38 & 30 & 21 & 3.7 & 79 & \\
\hline Co.015 & 146 & 124 & 11.7 & 8.6 & 94 & 94 \\
\hline Co.016 & 163 & 146 & 10.5 & 9.6 & 96 & 96 \\
\hline Co. 017 & 202 & 183 & 8.5 & 11.9 & 97 & 97 \\
\hline Co.018 & 185 & 165 & 9.2 & 11 & 97 & 94 \\
\hline
\end{tabular}


Table 4. $\mathrm{H}_{2}$ Chemisorption ${ }^{\mathrm{a}}$ and TPR Results (contd.)

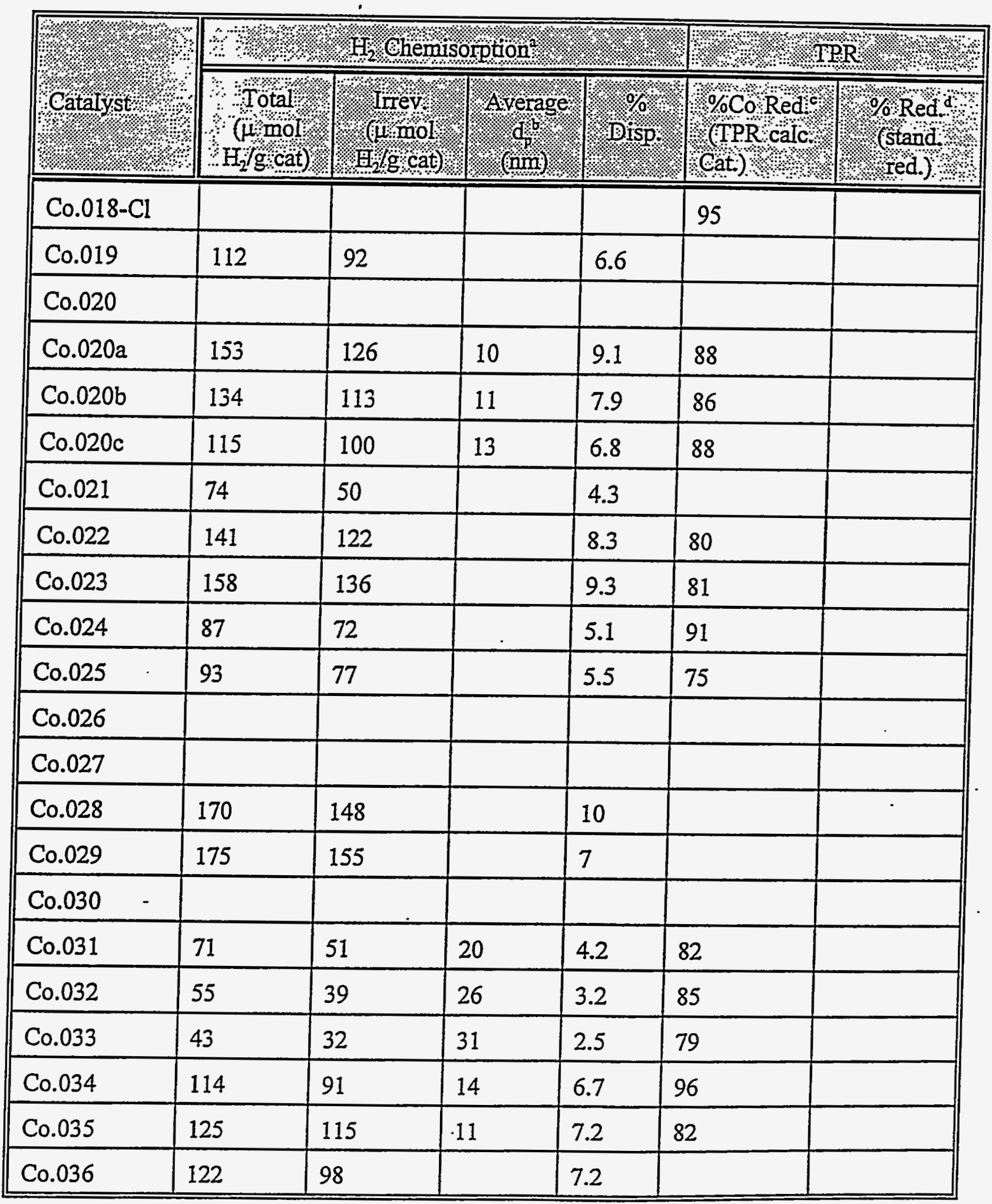


Table 4. $\mathrm{H}_{2}$ Chemisorption ${ }^{\mathrm{a}}$ and TPR Results (contd.)

\begin{tabular}{|c|c|c|c|c|c|c|}
\hline \multirow{2}{*}{ 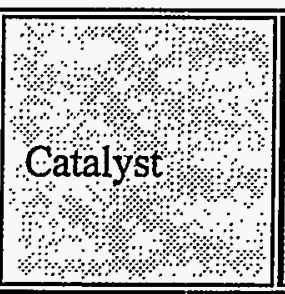 } & \multicolumn{6}{|c|}{ 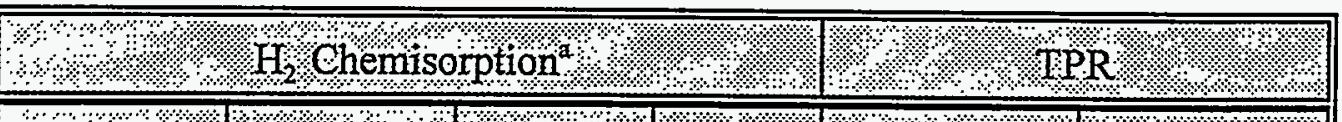 } \\
\hline & $\begin{array}{c}\text { Total } \\
(\mu \mathrm{mol} / \\
\left.\mathrm{H}_{2} / \mathrm{g} \text { cat }\right) \\
\end{array}$ & 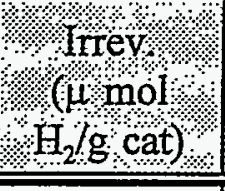 & 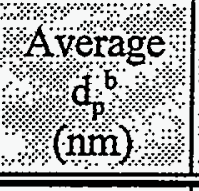 & \%isp? & 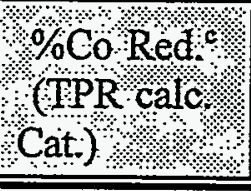 & $\begin{array}{l}\% \% \mathrm{Red} \\
\text { (stand } \\
\% \mathrm{red})\end{array}$ \\
\hline Co.037 & 21 & 3 & 48 & 2 & 99 & \\
\hline Co.038 & 45 & 35 & 21 & 4.3 & 91 & \\
\hline Co.039 & 19 & 16 & 40 & 1.8 & 72 & \\
\hline Co.040 & 14 & 11 & 53 & 1.4 & & \\
\hline Co.041 & 70 & 55 & & 4 & & \\
\hline Co.043 & 137 & 109 & & 8 & 86 & \\
\hline $\begin{array}{cc}C 0.053^{g} & (1) \\
& (2) \\
& (3) \\
\end{array}$ & $\begin{array}{l}168 \\
101 \\
138 \\
\end{array}$ & $\begin{array}{l}139 \\
76 \\
117 \\
\end{array}$ & $\begin{array}{l}8 \\
14 \\
10 \\
\end{array}$ & $\begin{array}{l}8.4 \\
5.2 \\
6.9 \\
\end{array}$ & $\begin{array}{l}60 \\
82 \\
47 \\
\end{array}$ & \\
\hline Co.054 & & & & & 57 & \\
\hline $\begin{array}{cc}\mathrm{Co.05}^{\mathrm{h}} & (1) \\
& (2) \\
& (3) \\
& (4) \\
\end{array}$ & $\begin{array}{l}165 \\
179 \\
103 \\
192 \\
\end{array}$ & $\begin{array}{l}139 \\
163 \\
84 \\
165\end{array}$ & $\begin{array}{l}8.5 \\
8 \\
14 \\
7\end{array}$ & $\begin{array}{l}8.3 \\
9.0 \\
5.2 \\
9.6\end{array}$ & $\begin{array}{l}78 \\
50 \\
76 \\
-\end{array}$ & \\
\hline Co.056 & & & & & 58 & \\
\hline CAL.01 & - & - & $58^{e}$ & $4.4^{e}$ & 54 & \\
\hline CAL.02 & - & - & $63^{e}$ & $5.1^{e}$ & 55 & \\
\hline CAL.03 & - & - & $57^{\mathrm{e}}$ & $4.2^{\mathrm{e}}$ & 85 & \\
\hline CAL.04 & & & $55^{\mathrm{e}}$ & $3.5^{\mathrm{c}}$ & & \\
\hline CAL.05 & & & $56^{e}$ & $4.4^{e}$ & & \\
\hline CAL.06 & & & $57^{\mathrm{e}}$ & $4.2^{e}$ & & \\
\hline CAL.10 & & & $72^{e}$ & $3.7^{\mathrm{e}}$ & & \\
\hline
\end{tabular}

(a) static $\mathrm{H}_{2}$ chemisorption at $100^{\circ} \mathrm{C}$

(b) Est. assuming $\mathrm{H}_{\mathrm{tot}} / \mathrm{Co}_{\mathrm{s}}=1,5.46 \times 10^{-20} \mathrm{~m}^{2} / \mathrm{Co}_{\mathrm{s}}$, and $\mathrm{d}_{\mathrm{p}}=5 / \mathrm{S}_{\mathrm{Co}} / \rho$

$\mathrm{S}_{\mathrm{Co}_{0}}$ is based on amt. Co reducible during standard reduction

(c) \% Co reducible from TPR of calcined catalysts up to $900^{\circ} \mathrm{C}$

(d) \% Co reduced after standard reduction procedure 
(e) Based on $\mathrm{CO}$ chemisorption

(f) (1)=uncalcined; (2)=calcined in air and reduced in $\mathrm{H}_{2}$; (3)=calcined in $\mathrm{He}$; (4)calcined in nitrogen

(g) (1) and (2)=uncalcined

(h) (1) and (3)=calcined in air; (2)=calcined in nitrogen; (4)=uncalcined

(i) Catalyst $\mathrm{Co} .018-\mathrm{Cl}$ is chlorinated. 
Table 5. Summary of fixed bed reaction results

\begin{tabular}{|c|c|c|c|c|c|c|c|c|c|c|c|c|c|}
\hline \%akxtsk & 19. & 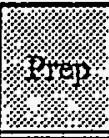 & 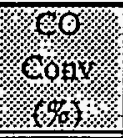 & .rones: & (18ch & 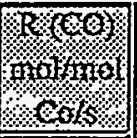 & 4 & 813tara & 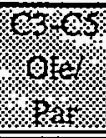 & $\sqrt{.89}$ & 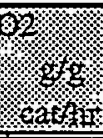 & r.t. & 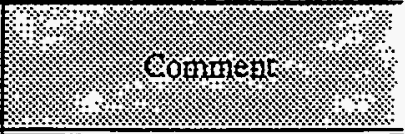 \\
\hline Co.001 & 4 & $\overline{(1)}$ & 2.6 & 0.116 & 0.056 & $\mid 6.8 \mathrm{E}-04$ & 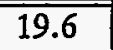 & $\overline{0.76}$ & $8.61^{\circ}$ & 0.4 & 0.061 & & NSH** \\
\hline $\mathrm{Co.002}$ & 4 & (2) & 6.7 & 0.258 & 0.082 & $1.5 \mathrm{E}-03$ & 26.1 & 0.64 & 1.97 & 0.1 & 0.012 & & NS \\
\hline Co.003 & 1 & (3) & 5.5 & 0.185 & 0.044 & $1.1 \mathrm{E}-03$ & 30.8 & 0.57 & 4.22 & & & 28.9 & old startup \\
\hline \multirow{11}{*}{$\begin{array}{c}\text { Co.004 } \\
n \\
n \\
n \\
n \\
n \\
n \\
n \\
\% \\
\%\end{array}$} & 1 & (2) $s$ & 4.3 & 0.291 & 0.086 & $1.7 \mathrm{E}-03$ & 30.4 & 0.62 & 2.77 & 0.1 & 0.025 & \multirow[t]{11}{*}{25.9} & NSH \\
\hline & 2 & $\mathrm{u}$ & 11.2 & 0.517 & 0.155 & $3.0 \mathrm{E}-03$ & 28.9 & 0.61 & 1.02 & 0.1 & 0.038 & & NSH \\
\hline & $2 \mathrm{~h}$ & $\mathrm{u}$ & 47.6 & 0.548 & 0.211 & $3.2 \mathrm{E}-03$ & 21.9 & 0.64 & 0.40 & 2.3 & 0.090 & & NSH, high conv. study \\
\hline & $2 a$ & $\mathrm{f}$ & 3.8 & 0.177 & 0.063 & $1.0 \mathrm{E}-03$ & 25.5 & 0.65 & 3.02 & 0.1 & 0.024 & & NSH, calc.after run2,T surge \\
\hline & 3 & $\mathbf{u}$ & 6.7 & 0.499 & 0.153 & $2.9 \mathrm{E}-03$ & 27.8 & 0.61 & 1.45 & 0.1 & 0.036 & & NSH \\
\hline & $3 \mathrm{~h}$ & u & 27.0 & 0.506 & 0.164 & $3.0 \mathrm{E}-03$ & 27.3 & 0.62 & 0.46 & 0.8 & 0.048 & & NSH, high conv. sudy \\
\hline & 4 & $\mathbf{u}$ & 1.8 & 0.427 & 0.241 & $2.5 \mathrm{E}-03$ & 24.0 & 0.79 & 2.40 & 0.1 & 0.078 & & $\mathrm{NSH}, 10 \mathrm{~atm}$ \\
\hline & 5 & $\mathrm{f}$ & 4.5 & 0.288 & 0.150 & $1.7 \mathrm{E}-03$ & 19.4 & 0.75 & 7.84 & 0.1 & 0.03 & & NSH \\
\hline & 6 & n & 6.9 & 0.466 & 0.151 & 2.7E-03 & 27.8 & 0.61 & 1.60 & 0.2 & 0.044 & & $\mathrm{NSH}$ \\
\hline & 7 & ROR & 3.9 & 0.225 & 0.072 & $1.3 \mathrm{E}-03$ & 28.8 & 0.61 & 3.01 & 0.2 & 0.042 & & $\mathrm{NSH}$ \\
\hline & 8 & $\mathrm{He}$ & 5.8 & 0.399 & 0.144 & $2.3 \mathrm{E}-03$ & 27.2 & 0.66 & 2.13 & 0.1 & 0.028 & & NSH \\
\hline \multirow{8}{*}{$\begin{array}{c}\text { Co.005 } \\
n \\
n \\
" \\
" \\
n \\
n \\
n\end{array}$} & 2 & \multirow[t]{8}{*}{ (1) } & 3.3 & .0 .087 & 0.037 & $5.1 E-04$ & 21.4 & 0.66 & 3.40 & $\overline{0.1}$ & 0.005 & \multirow{8}{*}{25.1} & NS \\
\hline & $2 a$ & & 2.5 & 0.066 & 0.021 & $3.9 \mathrm{E}-04$ & 25.9 & 0.59 & 4.92 & 0.1 & 0.004 & & 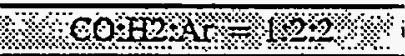 \\
\hline & $2 b$ & & 2.4 & 0.064 & 0.021 & 3.7E-04 & 26.3 & 0.60 & 6.22 & 0.1 & \multirow{6}{*}{$\begin{array}{l}0.000 \\
0.013 \\
0.009 \\
0.018 \\
0.017 \\
0.003\end{array}$} & & 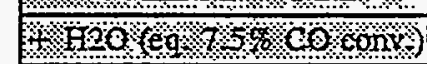 \\
\hline & 3 & & 3.3 & 0.077 & 0.024 & $4.5 \mathrm{E}-04$ & 28.4 & 0.62 & 2.29 & 0.1 & & & $\mathrm{NSH}$ \\
\hline & 4 & & 4.0 & 0.069 & 0.021 & $4.0 \mathrm{E}-04$ & 30.0 & 0.62 & 2.16 & 0.2 & & & NSH \\
\hline & 5 & & 3.9 & 0.171 & 0.084 & $1.0 \mathrm{E}-03$ & 26.8 & 0.76 & 2.59 & 0.1 & & & NSH, 10 atm \\
\hline & 6 & & 2.1 & 0.129 & 0.044 & $7.6 \mathrm{E}-04$ & 25.7 & 0.64 & 5.53 & 0.1 & & & $\mathrm{NSH}$ \\
\hline & 7 & & 6.7 & 0.084 & 0.040 & $4.9 \mathrm{E}-04$ & 18.8 & 0.70 & 1.95 & 0.1 & & & S2 \\
\hline Co.005a & 1 & $(4 a)$ & 3.2 & 0.227 & 0.067 & $1.3 \mathrm{E}-03$ & 30.0 & 0.60 & 3.05 & 0.1 & 0.034 & & $\mathrm{NSH}$ \\
\hline Co.005b & 1 & (1) & 2.4 & 0.040 & 0.024 & $2.3 E-04$ & 13.9 & 0.74 & 0.55 & 0.4 & 0.023 & & S2,1/4WGS.03+3/4Co.005 \\
\hline Co.005c & 1 & (1) & 5.7 & 0.071 & 0.031 & 4.1E-04 & 20.3 & 0.68 & 0.21 & 1.8 & 0.076 & & S2,1/2WGS.03+1/2Co.005 \\
\hline Co.006 & 1 & (5) & 2.7 & 0.052 & 0.007 & $5.1 \mathrm{E}-04$ & 45.0 & 0.49 & 1.90 & 0.1 & 0.006 & & NSH \\
\hline Co.007 & 1 & (5) & 3.2 & 0.024 & 0.003 & $1.4 \mathrm{E}-04$ & 49.8 & 0.50 & 0.72 & 0.2 & 0.004 & & NSH \\
\hline Co.009 & 1 & (1) & 4.9 & 0.346 & 0.090 & $2.0 \mathrm{E}-03$ & 31.1 & 0.57 & 1.69 & 0.1 & 0.020 & & NSH \\
\hline$"$ & 2 & & 4.9 & 0.338 & 0.091 & $2.0 \mathrm{E}-03$ & 31.1 & 0.58 & 1.64 & 0.1 & 0.026 & & NSH \\
\hline Co.010a & 1 & $(4 b) s$ & 3.5 & 0.246 & 0.076 & $1.4 \mathrm{E}-03$ & 29.5 & 0.62 & 2.58 & 0.2 & 0.040 & & NSH \\
\hline$"$ & 2 & & 4.1 & 0.262 & 0.080 & $1.5 \mathrm{E}-03$ & 30.0 & 0.61 & 2.31 & 0.1 & 0.022 & & NSH \\
\hline Co.011 & 2 & $(6 \mathrm{~b})$ & 2.9 & 0.094 & 0.037 & $5.5 \mathrm{E}-04$ & 22.4 & 0.61 & 4.83 & 0.3 & 0.030 & & $\mathrm{NSH}$ \\
\hline Co.012 & 1 & (1) & 3.8 & 0.083 & 0.027 & $4.8 \mathrm{E}-04$ & 26.3 & 0.64 & 4.40 & & & 22.5 & slow CO startup (60 min) \\
\hline " & 2 & & 4.4 & 0.085 & 0.030 & $5.0 \mathrm{E}-04 \|$ & 29.1 & 0.64 & 1.93 & 0.3 & 0.018 & & NSH \\
\hline$"$ & 3 & & 2.1 & 0.081 & 0.026 & $4.7 \mathrm{E}-04$ & 28.6 & 0.66 & 3.57 & 0.4 & 0.049 & & $\mathrm{NSH}$ \\
\hline$"$ & 4 & & 2.5 & 0.105 & 0.035 & $6.2 E-(1) 4$ & 28.4 & 0.61 & 4.94 & 0.4 & 0.023 & & $\mathrm{NSH}$ \\
\hline Cos.(1)14 & 1 & (1) & 1.5 & 0.028 & 0.009 & $2.8 \mathrm{E}-() 4$ & 32.3 & 0.63 & 4.70 & 0.1 & 0.003 & & NSH \\
\hline$"$ & 2 & & 2.6 & 0.034 & 0.013 & 3.3E-04 & 27.7 & 0.69 & 10.8 & 0.1 & 0.011 & 26.0 & $\mathrm{NSH}$ \\
\hline Co.015 & 1 & (4) $: \mathrm{s}$ & 6.8 & 0.211 & 0.063 & $1.2 \mathrm{E}-() 3$ & 26.7 & 0.60 & 3.30 & 0.1 & 0.011 & & slow CO starcup (120 min) \\
\hline$"$ & 2 & $t^{t}$ & $7.1)$ & 0.388 & 0.135 & 2.3E- 03 & 26.4 & 0.64 & 1.39 & 0.1 & 0.011 & & $\mathrm{NSH}$ \\
\hline$\%$ & 3 & $u$ & 6.7 & 0.375 & 0.123 & $2.2 \mathrm{E}-03$ & 28.2 & 0.63 & 1.62 & 0.2 & 0.011 & & $\mathrm{NSH}$ \\
\hline Co.016 & 1 & (4a) $s$ & 5.2 & 0.161 & 0.035 & $9.4 \mathrm{E}-04$ & 33.6 & 0.56 & 6.85 & & & & slow CO starup (30 min) \\
\hline$n$ & 2 & $\mathrm{f}$ & 5.1 & 0.291 & 0.112 & $1.7 E-03$ & 25.1 & 0.68 & 2.48 & 0.1 & 0.01 & & $\mathrm{NSH}$ \\
\hline
\end{tabular}


Table 5. Summary of fixed bed reaction results (contd.)

\begin{tabular}{|c|c|c|c|c|c|c|c|c|c|c|c|c|c|}
\hline कán & Rition & \% & \%op & 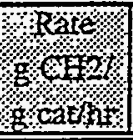 & 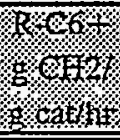 & Romolo & w & (3) & א.3. & \multicolumn{2}{|c|}{ 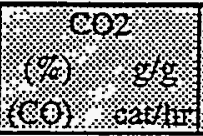 } & \%od & eonineg \\
\hline C0.017 & 2 & (1) $s$ & $\overline{4.6}$ & 0.139 & 0.066 & $8.2 \mathrm{E}-04$ & 22.0 & 0.69 & 3.72 & 0.1 & 0.012 & & NS \\
\hline$n$ & $2 a$ & $s$ & 3.7 & 0.111 & 0.043 & $6.5 \mathrm{E}-04$ & 25.9 & 0.64 & 4.19 & 0.1 & 0.011 & & 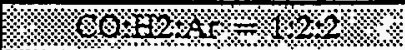 \\
\hline$n$ & $2 \mathrm{~b}$ & $s$ & 3.4 & 0.102 & 0.039 & $6.0 \mathrm{E}-04$ & 26.9 & 0.65 & 4.39 & 0.1 & 0.013 & & 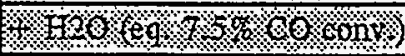 \\
\hline \%৷ & 3 & $\mathfrak{f}$ & 4.9 & 0.274 & 0.111 & $1.6 \mathrm{E}-03$ & 23.0 & 0.67 & 2.41 & 0.2 & 0.030 & & NSH, calc. flow air \\
\hline Co.018 & 2 & $\mathbf{s}$ & 3.4 & 0.170 & 0.068 & $1.0 \mathrm{E}-03$ & 27.2 & 0.69 & 2.24 & 0.1 & 0.013 & & NS \\
\hline$"$ & $2 a$ & s & 2.7 & 0.133 & 0.041 & $7.8 \mathrm{E}-04$ & 33.2 & 0.64 & 2.52 & 0.1 & 0.011 & & 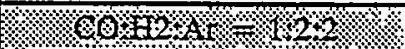 \\
\hline$n$ & $2 \mathrm{~b}$ & $s$ & 3.0 & 0.147 & 0.045 & $8.6 \mathrm{E}-04$ & 33.6 & 0.63 & 2.41 & 0.1 & 0.015 & & 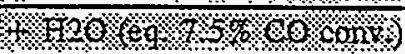 \\
\hline$n$ & 3 & $s$ & 4.1 & 0.290 & 0.086 & $1.7 \mathrm{E}-03$ & 30.0 & 0.62 & 2.34 & 0.1 & 0.022 & & NSH \\
\hline " & 4 & $s$ & 3.6 & 0.340 & 0.180 & $2.0 E-03$ & 25.4 & 0.80 & 1.92 & 0.1 & 0.041 & & $\mathrm{NSH}, 10 \mathrm{~atm}$ \\
\hline " & 5 & $\mathrm{t}$ & 7.5 & 0.470 & 0.136 & $2.8 \mathrm{E}-03$ & 29.0 & 0.60 & 1.93 & 0.3 & 0.055 & & NSH \\
\hline " & 6 & $\mathrm{f}$ & 6.5 & 0.409 & 0.147 & $2.4 \mathrm{E}-03$ & 24.6 & 0.64 & 2.42 & & & & NSH,S2" \\
\hline $\mathrm{Co.018 \textrm {Cl }}$ & 1 & (1) & 4.5 & 0.320 & 0.075 & $1.9 \mathrm{E}-03$ & 33.5 & 0.57 & 1.45 & 0.1 & 0.018 & & NSH \\
\hline Co.019 & 1 & (1)s & 4.4 & 0.088 & 0.047 & $5.2 \mathrm{E}-04$ & 15.2 & 0.74 & 7.61 & & & & NS \\
\hline$"$ & 2 & $s$ & 2.2 & 0.085 & 0.046 & $5.0 \mathrm{E}-04$ & 18.9 & 0.73 & 8.59 & 0.3 & 0.040 & & $\mathrm{NSH}$ \\
\hline n & 3 & $\mathrm{f}$ & 3.4 & 0.142 & 0.071 & $8.3 E-04$ & 19.3 & 0.72 & 6.74 & 0.2 & 0.025 & & $\overline{\mathrm{NSH}}$ \\
\hline$"$ & $3 \mathrm{~h}$ & $\mathrm{t}$ & 16.7 & 0.172 & 0.091 & $1.0 \mathrm{E}-03$ & 18.2 & 0.74 & 1.77 & 1.0 & 0.034 & & NSH, high conv \\
\hline Co.020a & 1 & (4) $s$ & 4.6 & 0.343 & 0.085 & $2.0 \mathrm{E}-03$ & 33.5 & 0.57 & 1.70 & 0.1 & 0.024 & & $\mathrm{NSH}$ \\
\hline$"$ & 2 & & 4.4 & 0.315 & 0.086 & $1.8 \mathrm{E}-03$ & 31.2 & 0.59 & 1.96 & 0.1 & 0.028 & & $\mathrm{NSH}$ \\
\hline$\overline{C o .020 b}$ & 1 & (4b) $s$ & 4.4 & 0.311 & 0.084 & $1.8 \mathrm{E}-03$ & 31.7 & 0.58 & 1.81 & 0.2 & 0.051 & & NSH \\
\hline Co.020c & 1 & (4a) $\mathrm{s}$ & 4.0 & 0.287 & 0.083 & $1.7 \mathrm{E}-03$ & 30.4 & 0.60 & 2.38 & 0.1 & 0.017 & & $\mathrm{NSH}$ \\
\hline Co.021 & 1 & (6b) & 3.4 & 0.109 & 0.032 & $6.4 \mathrm{E}-04$ & 27.3 & 0.62 & 2.78 & 0.1 & 0.012 & 28.2 & $\mathrm{NSH}$ \\
\hline$n$ & 2 & & 3.6 & 0.114 & 0.035 & 6.7E-04 & 28.0 & 0.56 & 2.69 & 0.1 & 0.012 & & $\mathrm{NSH}$ \\
\hline Co.022 & 1 & (6c) & 3.8 & 0.121 & 0.037 & $7.1 \mathrm{E}-04$ & 26.7 & 0.55 & 2.91 & 0.1 & 0.015 & 30.1 & NSH \\
\hline Co.023 & 1 & $(6 c)$ & 3.8 & 0.123 & 0.038 & $7.2 \mathrm{E}-04$ & 28.3 & 0.56 & 3.18 & 0.2 & 0.023 & 29.5 & $\mathrm{NSH}$ \\
\hline Co.024 & 1 & $(7)$ & 5.1 & 0.165 & 0.047 & 9.7E-04 & 32.8 & 0.62 & 2.08 & 0.5 & 0.055 & & $\mathrm{NSH}$ \\
\hline$"$ & 2 & & 5.7 & 0.182 & 0.060 & $1.1 \mathrm{E}-03$ & 28.7 & 0.62 & 2.25 & 0.3 & 0.035 & & NSH \\
\hline Co.025 & 1 & (4) & 5.0 & 0.160 & 0.062 & $9.4 \mathrm{E}-04$ & 23.5 & 0.63 & 4.13 & 0.2 & 0.019 & 26.9 & $\mathrm{NSH}$ \\
\hline Co.026 & 1 & (1) & 4.4 & 0.129 & 0.043 & $5.0 \mathrm{E}-04$ & 26.9 & 0.62 & 2.65 & 0.2 & 0.022 & & $\mathrm{NSH}$ \\
\hline$"$ & in & & 22.9 & 0.170 & 0.072 & $6.6 \mathrm{E}-04$ & 22.6 & 0.67 & 0.64 & 1.5 & 0.037 & & NSH, high conv. study \\
\hline $\bar{C} 0.027$ & 1 & (1) & 4.2 & 0.169 & 0.068 & $6.6 \mathrm{E}-04$ & 23.0 & 0.65 & 3.44 & 0.2 & 0.026 & & NSH \\
\hline$"$ & $1 \mathrm{~h}$ & & 17.8 & 0.180 & 0.085 & 7.0E-04 & 19.4 & 0.71 & 0.96 & 1.2 & 0.011 & & NSH, high conv. study \\
\hline$\overline{C o .028}$ & 1 & (4) & 3.8 & 0.146 & 0.076 & $5.7 \mathrm{E}-04$ & 18.2 & 0.76 & 7.63 & 0.2 & 0.031 & & NSH \\
\hline " & 2 & & 4.3 & 0.167 & 0.105 & $6.5 \mathrm{E}-04$ & 14.7 & 0.80 & 13.2 & 0.2 & 0.030 & 28.4 & NSH \\
\hline " & 3 & & 4.5 & 0.206 & 0.126 & 8.0E- 04 & 15.5 & 0.79 & 13.4 & 0.2 & 0.037 & & NSH, re-calc. in flow air \\
\hline C0.(129) & 1 & (4) & 2.8 & 0.173 & 0.105 & $6.7 E-(04$ & 15.3 & 0.79 & 25.9 & 0.2 & 0.026 & & $\mathrm{NSH}$ \\
\hline$C_{0.0131}$ & 1 & (1) & 1.9 & 0.060 & 0.022 & $3.5 \mathrm{E}-(1) 4$ & 26.1 & 0.64 & 4.65 & 0.3 & 0.031 & & $\mathrm{NSH}$ \\
\hline Co.(1)32 & 1 & (1) & 3.3 & 0.183 & 0.079 & $1.1 \mathrm{E}-(1) 3$ & 22.0 & 0.70 & 5.28 & 0.1 & 0.022 & & $\mathrm{NSH}$ \\
\hline$C_{0.0133}$ & 1 & (1) & 1.3 & 0.073 & 0.027 & $4.3 \mathrm{E}-(1) 4$ & 24.1 & 0.67 & 7.36 & 0.1 & 0.020 & & $\mathrm{NSH}$ \\
\hline C(0.0)34 & 1 & (4) & 5.0 & 0.275 & 0.107 & $1.6 \mathrm{E}-(03)$ & 24.0 & 0.67 & 4.29 & 0.1 & 0.018 & & $\mathrm{NSH}$ \\
\hline C0.01035 & 2 & (6) & 4.6 & 0.147 & 0.068 & $8.6 \mathrm{E}-04$ & 22.0 & 0.69 & 4.84 & 0.2 & 0.019 & & NSH \\
\hline Co.1136 & 2 & (6c) & 3.9 & 0.125 & 0.045 & $7.3 \mathrm{E}-04$ & 28.9 & 0.67 & 4.20 & 0.1 & 0.013 & & $\mathrm{NSH}$ \\
\hline Co.0137 & 1 & (4a) & 2.7 & 0.053 & 0.011 & $5.2 \mathrm{E}-04$ & 32.8 & 0.59 & 2.82 & & & & $\mathrm{NSH}$ \\
\hline$\overline{C 0 .(1) 38}$ & 1 & (4a) & 4.5 & 0.051 & 0.018 & $4.9 \mathrm{E}-04$ & 25.1 & 0.68 & 4.63 & 0.3 & 0.013 & 24.2 & $\mathrm{NSH}$ \\
\hline
\end{tabular}


Table 5. Summary of fixed bed reaction results (contd.)

\begin{tabular}{|c|c|c|c|c|c|c|c|c|c|c|c|c|c|}
\hline 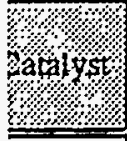 & Rü & $12+18 \%$ & 8 & 6 & $8 \%$ & $1701 / 716$ & ky & aeptiat & \%olos & (6) & 8. & 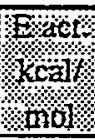 & onment \\
\hline 30.039 & 1 & (5) & 1.1 & 0.021 & 0.004 & $2.0 \mathrm{E}-04$ & 57.2 & 0.64 & 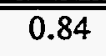 & 0.2 & 0.010 & \multirow[t]{2}{*}{25.6} & NSH, cracking in the line \\
\hline$"$ & 2 & & 3.9 & 0.049 & 0.007 & 4.7E-04 & 42.4 & 0.48 & 0.95 & 0.2 & 0.009 & & NSH \\
\hline 0.040 & 1 & (4a) & 1.9 & 0.037 & 0,004 & $3.7 \mathrm{E}-04$ & 45.2 & 0.53 & 1.79 & 0.2 & 0.007 & & $\mathrm{NSH}$ \\
\hline$"$ & 2 & & 2.8 & 0.033 & 0.004 & 3.3E-04 & 44.6 & 0.46 & 1.12 & 0.2 & 0.007 & & $\mathrm{NSH}$ \\
\hline 0.041 & 1 & (4) & 3.5 & 0.136 & 0.042 & 8.0E-04 & 40.7 & 0.69 & 1.47 & 0.6 & 0.079 & & NSH, cracking in the line \\
\hline$\overline{0.043}$ & 1 & (4a) & 4.6 & 0.104 & 0.062 & $6.1 \mathrm{E}-04$ & 15.3 & 0.78 & 12.1 & 0.6 & \multirow{2}{*}{$\begin{array}{l}0.048 \\
0.035\end{array}$} & \multirow[b]{2}{*}{35.1} & \multirow{2}{*}{$\frac{\mathrm{NSH}, 2.5 \mathrm{hr} \text { on-stream }}{\mathrm{NSH}}$} \\
\hline$"$ & 2 & & 3.7 & 0.078 & 0.045 & $4.6 \mathrm{E}-04$ & 16.3 & 0.78 & 7.75 & 0.5 & & & \\
\hline 00044 & 1 & (4) & 3.1 & 0.179 & 0.081 & 1.0E-03 & 22.7 & 0.73 & 5.16 & 0.3 & 0.020 & & $\mathrm{NSH}$ \\
\hline $30 \% 15$ & 1 & (4) & 2.6 & 0.161 & 0.076 & $9.4 \mathrm{E}-04$ & 22.2 & 0.73 & 6.89 & 0.3 & 0.022 & & $\mathrm{NSH}$ \\
\hline 0.047 & 1 & (4) & 6.4 & 0.263 & 0.128 & $1.5 \mathrm{E}-03$ & 21.3 & 0.73 & 7.20 & 0.3 & 0.040 & 26.6 & NSH \\
\hline 0.048 & 1 & (4) & 3.5 & 0.162 & 0.094 & $9.5 \mathrm{E}-04$ & 16.3 & 0.77 & 17.1 & 0.2 & 0.027 & 28.0 & $\mathrm{NSH}$ \\
\hline 0.049 & 1 & (4) & 7.1 & 0.366 & 0.153 & $2.1 \mathrm{E}=03$ & 23.4 & 0.69 & 5.63 & 0.3 & 0.043 & & NSH \\
\hline " & 2 & & 6.0 & 0.388 & 0.160 & $2.3 \mathrm{E}-03$ & 23.3 & 0.68 & 5.93 & 0.3 & 0.056 & & NSH, re-calcined in flow air \\
\hline 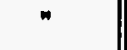 & 3 & $f$ & 6.7 & 0.410 & 0.158 & $2.4 \mathrm{E}-03$ & 25.2 & 0.69 & 4.64 & 0.3 & 0.059 & & NSH \\
\hline$"$ & $3 \mathrm{~h}$ & $f$ & 44.9 & 0.457 & 0.178 & $2.7 \mathrm{E}-03$ & 26.5 & 0.67 & 0.67 & 3.8 & 0.131 & & NSH,HCS \\
\hline$: 0.053$ & 1 & (1) & 7.0 & 0.408 & 0.127 & $2.4 \mathrm{E}-03$ & 27.3 & 0.62 & 3.07 & 0.2 & 0.049 & & $\mathrm{NSH}$ \\
\hline " & Ih & & 28.5 & 0.417 & 0.145 & $2.4 \mathrm{E}-03$ & 26.8 & 0.64 & 0.79 & 1.6 & 0.081 & & NSH, high conv. study \\
\hline n & 2 & f & 6.6 & 0.433 & 0.174 & $2.5 \mathrm{E}-03$ & 23.4 & 0.63 & 2.35 & 0.2 & $0.052 \mid$ & & NSH \\
\hline n & 3 & & 7.8 & 0.453 & 0.143 & $2.7 \mathrm{E}-03$ & 27.6 & 0.62 & 1.67 & 0.1 & 0.027 & & NSH \\
\hline n & $3 \mathrm{~h}$ & & 31.5 & 0.457 & 0.164 & $2.7 \mathrm{E}-03$ & 25.4 & 0.65 & 0.52 & 0.9 & 0.044 & & NSH, high conv. study \\
\hline & 4 & $\mathbf{u}$ & 6.1 & 0.426 & 0.142 & $2.5 \mathrm{E}-03$ & 28.3 & 0.65 & 1.41 & 0.1 & 0.030 & & NSH \\
\hline & 5 & ROR & 5.6 & 0.360 & 0.118 & $2.1 \mathrm{E}-03$ & 27.9 & 0.64 & 1.49 & 0.2 & 0.034 & & $\mathrm{NSH}$ \\
\hline $2 \%$ & 6 & $f$ & 8.2 & 0.485 & 0.163 & $2.8 \mathrm{E}-03$ & 28.9 & 0.64 & 1.25 & 0.1 & 0.030 & & NSH \\
\hline .0 .054 & 1 & (1) & 1.2 & 0.041 & 0.023 & $2.4 \mathrm{E}-04$ & 17.8 & 0.79 & 16.3 & 0.2 & 0.014 & & NSH \\
\hline 0.055 & 1 & (1) $f$ & 9.1 & 0.490 & 0.159 & $2.9 \mathrm{E}-03$ & 28.9 & 0.63 & 1.31 & 0.2 & 0.034 & & $\mathrm{NSH}$ \\
\hline " & 2 & u & 4.9 & 0.290 & 0.105 & 1.7E-03 & 25.7 & 0.65 & 1.49 & 0.2 & 0.022 & & NSH, T surge \\
\hline$"$ & 3 & $u$ & 11.0 & 0.611 & 0.193 & $3.6 \mathrm{E}-03$ & 29.4 & 0.60 & 1.02 & .0 .2 & 0.033 & & $\mathrm{NSH}$ \\
\hline \% & 4 & $\mathrm{u}$ & 5.9 & 0.380 & 0.124 & $2.2 \mathrm{E}-03$ & 28.3 & 0.60 & 1.94 & 0.2 & 0.047 & & NSH \\
\hline & 5 & $\mathrm{u}$ & 6.6 & 0.409 & 0.135 & 2.4E-03 & 29.6 & 0.59 & 1.83 & 0.2 & 0.030 & & $\mathrm{NSH}$ \\
\hline & 6 & ROR & 7.5 & 0.469 & 0.145 & 2.7E-03 & 30.9 & 0.63 & 1.21 & 0.2 & 0.023 & & $\mathrm{NSH}$ \\
\hline 乡y & 7 & $\mathrm{n}$ & 7.5 & 0.466 & 0.119 & $2.7 \mathrm{E}-03$ & 33.5 & 0.60 & 1.11 & 0.2 & 0.031 & & NSH \\
\hline $0.56 \%$ & 1 & (1) & 2.4 & 0.137 & 0.054 & $8.0 \mathrm{E}-04$ & 23.9 & 0.69 & 8.22 & 0.1 & 0.029 & & $\mathrm{NSH}$ \\
\hline$\%$ & 2 & $\underline{u}$ & 2.2 & 0.137 & 0.054 & $8.0 \mathrm{E}-04$ & 23.4 & 0.68 & 9.44 & 0.1 & 0.033 & & $\mathrm{NSH}$ \\
\hline $0,05 \%$ & 1 & (1) & 2.8 & 0.171 & 0.078 & $1.0 \mathrm{E}-03$ & 23.4 & 0.73 & 4.65 & 0.1 & 0.029 & & $\mathrm{NSH}$ \\
\hline 0060 & 1 & (2) & 3.8 & 0.244 & 0.097 & $1.4 \mathrm{E}-03$ & 25.0 & 0.69 & 3.82 & 0.1 & 0.023 & & $\mathrm{NSH}$ \\
\hline $4 L .01$ & 1 & (1) & 4.8 & 0.181 & 0.068 & $1.1 \mathrm{E}-03$ & 24.4 & 0.66 & 5.86 & 0.3 & 0.036 & & NSH, temp. surge (startup) \\
\hline$"$ & 2 & & 7.4 & 0.491 & 0.142 & $2.9 \mathrm{E}-03$ & 28.7 & 0.61 & 2.06 & 0.4 & 0.083 & & NSH \\
\hline " & 3 & & 2.5 & 0.164 & 0.053 & $9.6 \mathrm{E}-04$ & 25.6 & 0.63 & 4.97 & 0.2 & 0.052 & & NSH, HT, re-reduced \\
\hline$n$ & 4 & & 7.3 & 0.483 & 0.103 & $2.8 \mathrm{E}-03$ & 35.8 & 0.57 & 1.21 & 0.2 & 0.098 & & NSH, HT, re-calcined \\
\hline $1 L .02$ & 1 & (1) & 6.1 & 0.379 & 0.121 & $2.2 \mathrm{E}-03$ & 27.3 & 0.63 & 3.04 & 0.2 & 0.040 & & NSH \\
\hline IL.03 & 1 & (1) & 7.3 & 0.459 & 0.133 & $2.7 \mathrm{E}-03$ & 29.0 & 0.60 & 2.59 & 0.2 & 0.048 & & NSH \\
\hline$\underline{I L .04}$ & 1 & (1) & 4.1 & 0.234 & 0.123 & $1.4 \mathrm{E}-03$ & 19.1 & 0.71 & 8.98 & 0.2 & 0.026 & & $\mathrm{NSH}$ \\
\hline $1 \mathrm{IL.05}$ & 1 & (1) & 4.7 & 0.274 & 0.134 & $1.6 \mathrm{E}-03$ & 20.8 & 0.75 & 6.85 & 0.2 & 0.026 & & NSH \\
\hline
\end{tabular}




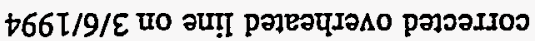

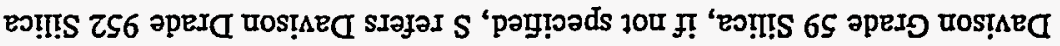

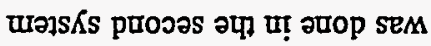
เәтит рәтеәप 'dmreis MәN dnareas MoN

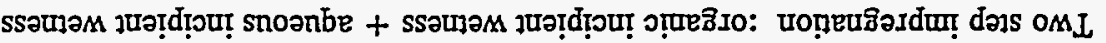

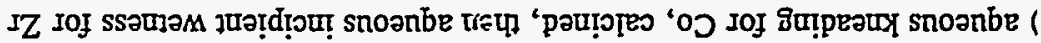

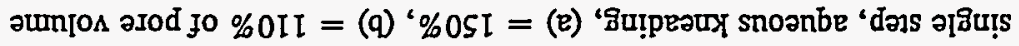

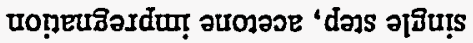

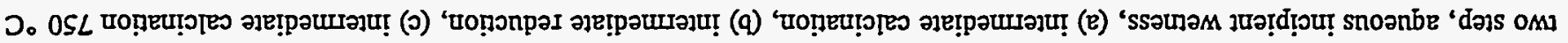

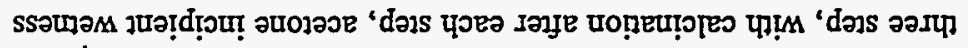

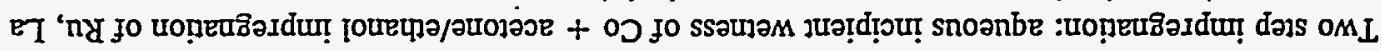

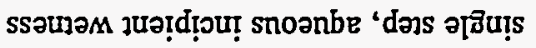

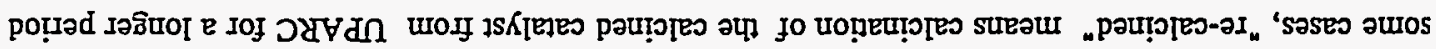

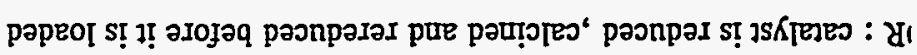

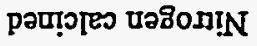

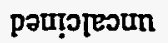
MOT ' ว!̣e?s '

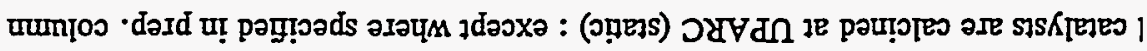

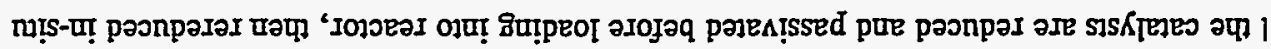

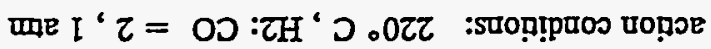

\begin{tabular}{|c|c|c|c|c|c|c|c|c|c|c|c|c|c|}
\hline HSN & & $\varepsilon 20^{\circ} 0$ & $\overline{\tau 0}$ & $6 \tau^{\prime} I$ & $19^{\circ} 0$ & $9 \cdot \tau \tau$ & $\varepsilon 0-B L I$ & toI:0 & $862^{\circ} 0$ & $8^{\circ} \mathrm{L}$ & (I) & I & don \\
\hline 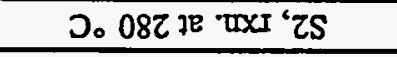 & & $\angle 0^{\circ} 0$ & $L^{\circ} I$ & $\angle E^{\circ} 0$ & $\tau \varepsilon^{\circ} 0$ & $\angle .89$ & $\varepsilon 0-\mathrm{g} \tau^{*} \mathrm{I}$ & $500^{\circ} 0$ & $\angle O Z^{\circ} 0$ & $0 . \angle I$ & & $\tau$ & 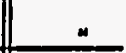 \\
\hline $2 S$ & & $210^{\circ} 0$ & $\varepsilon 0$ & $66^{\circ} 0$ & $96^{\circ} 0-$ & $\varepsilon \cdot 6 \varepsilon$ & to- $36^{\circ} \mathrm{I}$ & $500^{\circ} 0$ & $\tau \varepsilon 0^{\circ} 0$ & $9{ }^{\circ} \mathrm{Z}$ & $\mathrm{n}$ & $\tau$ & 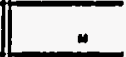 \\
\hline D. OSZ JE paonpar 'ZS & & $\angle 00^{\circ} 0$ & $\tau: 0$ & $I \tau \cdot \tau$ & $65^{\circ} 0$ & 0.92 & to-atr & $210^{\circ} 0$ & It0.0 & $\varepsilon \varepsilon$ & $(\mathrm{Bt})$ & I & $90^{\circ} \mathrm{MO}^{\circ}$ \\
\hline $2 S$ & & $600^{\circ} 0$ & $\tau \cdot 0$ & $\$ 8^{\circ} 0$ & $05^{\circ} 0$ & $8^{\circ} \hbar \varepsilon$ & $\neq 0-\exists t^{\circ} \varepsilon$ & II0.0 & $850^{\circ} 0$ & $L$ & $\mathbf{n}$ & $\tau$ & $\omega$ \\
\hline D. OSE JE paวnpaI 'ZS & & $800^{\circ} 0$ & $\tau * 0$ & $99^{\circ} 0$ & $55^{\circ} 0$ & $\tau \cdot \varepsilon \varepsilon$ & $b 0-\exists I \cdot z$ & $900^{\circ} 0$ & ऽEO०0 & $8 \cdot \tau$ & $(0 t)$ & $I$ & $50^{\circ} \mathrm{MO}^{\circ}$ \\
\hline 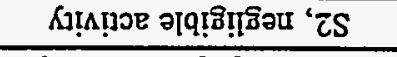 & & & & & & & & & & & $\left(E_{t}\right)$ & I & $50^{\circ} \mathrm{MO}^{\circ}$ \\
\hline 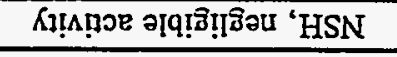 & & & & & & & & & & & $(8 t)$ & I & $\mathrm{EO}^{\circ} \mathrm{MO}^{\circ}$ \\
\hline J. 0Ec Je раэпраI 'HSN & & $8000^{\circ} 0$ & {$\left[{ }^{\circ} 0\right.$} & $t 6^{\circ} t$ & $09^{\circ} 0$ & $9^{\circ} \varsigma \bar{c}$ & S0-3b.5 & 200.0 & $600^{\circ} 0$ & $\varepsilon 0$ & $\left(B_{b}\right)$ & I & $20 \mathrm{MO}^{-}$ \\
\hline D. OSE IR paวnpaI "HSN & & $900^{\circ} 0$ & $1 \%$ & $\varepsilon^{*} I I$ & IS 0 & 6.06 & $s 0-\not b b^{\circ} \varepsilon$ & $000^{\circ} 0$ & $900^{\circ} 0$ & $\tau \cdot 0$ & & $\tau$ & “ \\
\hline כ. 0SE JE paวnpaI-әI 'HSN & & $\varepsilon 00^{\circ} 0$ & 10 & $\left.\$ 6^{\circ}\right)$ & $15^{\circ} 0$ & $I$ It & | $50-\exists \nabla^{\circ} \varepsilon$ & $100^{\circ} 0$ & $\angle 00^{\circ} 0$ & $\varsigma^{\circ} 0$ & & I & " \\
\hline כ. 0EC JE paOnpas 'HSN & & $9000^{\circ}$ & $\ddot{20}$ & $8 L^{\circ} \varepsilon$ & $85^{\circ} 0$ & $\tau \cdot 0 \varepsilon$ & $50-35 \cdot 6$ & $\varepsilon 00^{\circ} 0$ & $910^{\circ} 0$ & $\tau \cdot I$ & $\left(e_{t}\right)$ & 1 & $10^{\circ} \mathrm{MO}=$ \\
\hline HSN & & $5 \div 0^{\circ} 0$ & $\tau^{\circ} 0$ & $S L^{\circ} I$ & $\nabla L^{\circ} 0$ & $\varsigma^{\cdot} \varepsilon \tau$ & $E 0-\overline{G I} I$ & $\angle 60^{\circ} 0$ & $96 I^{\circ} 0$ & $\varsigma^{\cdot} \varepsilon$ & (I) & 1 & $01 \% \mathrm{O}$ \\
\hline HSN & & $120 \%$ & $\bar{\tau} 0$ & $05^{\circ} \mathrm{S}$ & $\forall \angle \circ 0$ & $\angle \cdot 0 Z$ & $\varepsilon 0-\bar{G} 9^{\circ} I$ & SEI 0 & $\angle 9 Z^{\circ} 0$ & $8^{\circ} \mathrm{S}$ & $(\mathrm{I})$ & $\mathrm{I}$ & $90^{\circ} \mathrm{THS}^{2}$ \\
\hline (1. & 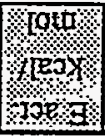 & $4 \%$ & 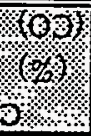 & 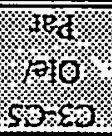 & 9 & \% & 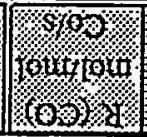 & 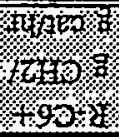 & fertor. & \%, & 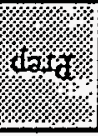 & ond & $\%$ \\
\hline
\end{tabular}


Table 6

DATE: $12 / 30 / 94$

COMPARISON OF CONVERSION AND SELECTIVITY OF

METHANE REDUCTION CATALYSTS AT STARTUP CONDITIONS

\begin{tabular}{cccc} 
Period No. & Temp. & Pres. & H2/CO Ratio \\
\hline 1 & $240 \mathrm{C}$ & $450 \mathrm{psi}$ & 2.0
\end{tabular}

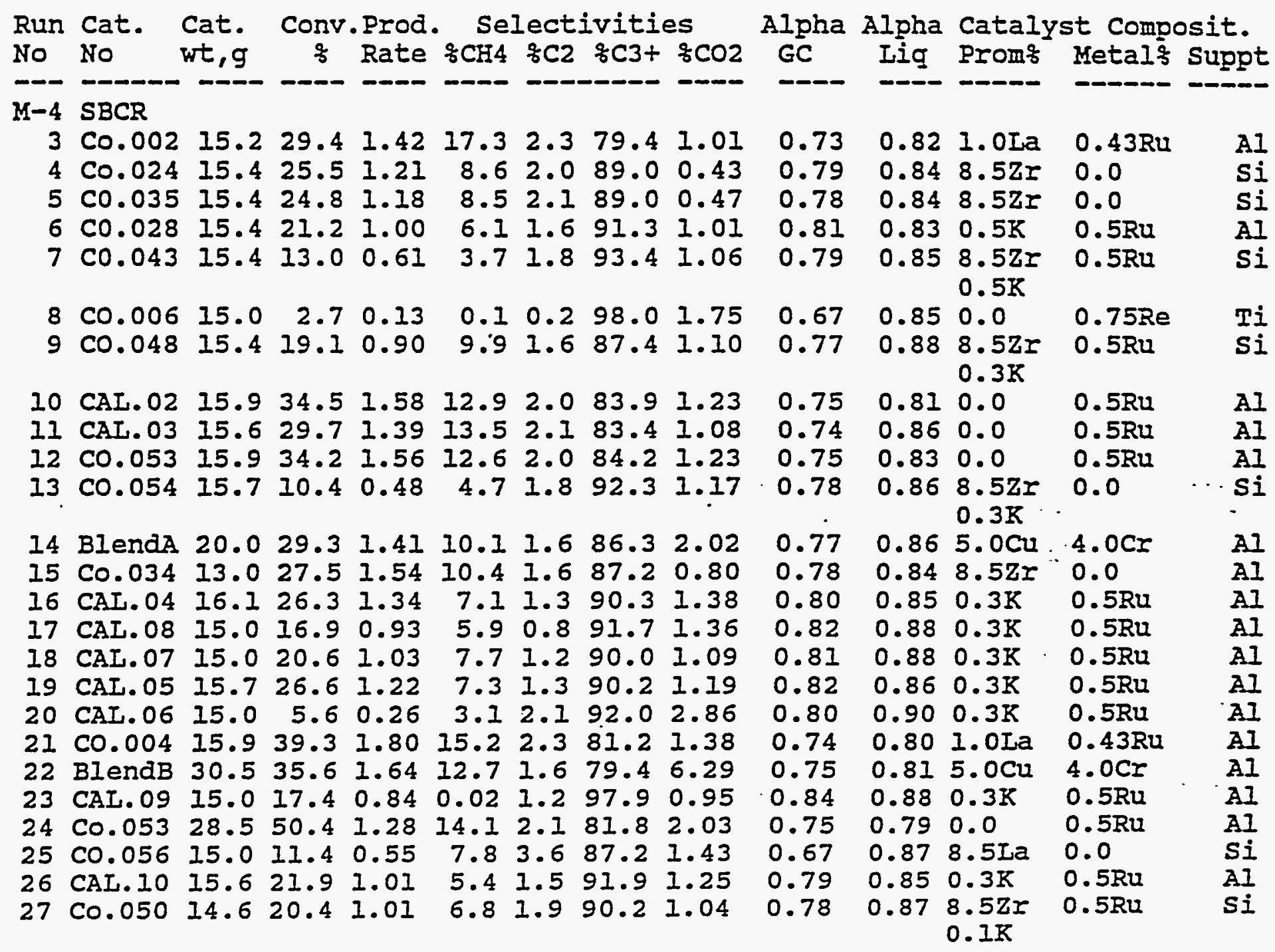

Note: Catalysts for all runs except Run 8 screened thru $150 \times 400$ mesh. Catalyst for Run 8 screened thru $100 \times 400$ mesh.

Blend A contains $15.0 \mathrm{gm}$. of Cat. No. Co.005 plus $5.0 \mathrm{gm}$

of Cat. No. WGS.03.

Cat. Nos. CAL.07 and CAL.08 were prereduced and wax coated.

H2 to CO ratio for Runs $16^{\circ}$ and 17 was $1.76 / 1.0$.

cat. No. CAL.06 was H2 reduced and air stabilized.

Blend B contains $15.0 \mathrm{gm}$ of Cat. No. Co.005 plus $15.0 \mathrm{gm}$

of Cat. No. WGS.03.

Cat. No. CAI.09 was prereduced and coated with soya.

Cat. No. CAI.10 was prepared on Condea Alumina. 
SUHMARY OF H3 SBCR RUH RESULTS

a) Unless otherwise noted, total flow is ca.15 L/min. STP, or $3 \mathrm{~cm} / \mathrm{sec}$ linear gas flow. b) Conversion is total $\mathrm{CO}$ conversion over the period $(x)$.

c)Conversion and selectivities are calculated using $\mathrm{K2}$ as an internal standard in the GC analysis of the offgas.

d)Prod. rate: Rate for protuction of total hydrocarbons,Ci+ (kg/kg cat., hr).

e)Alpha: Based on GC analysis of offgas and liquid product.

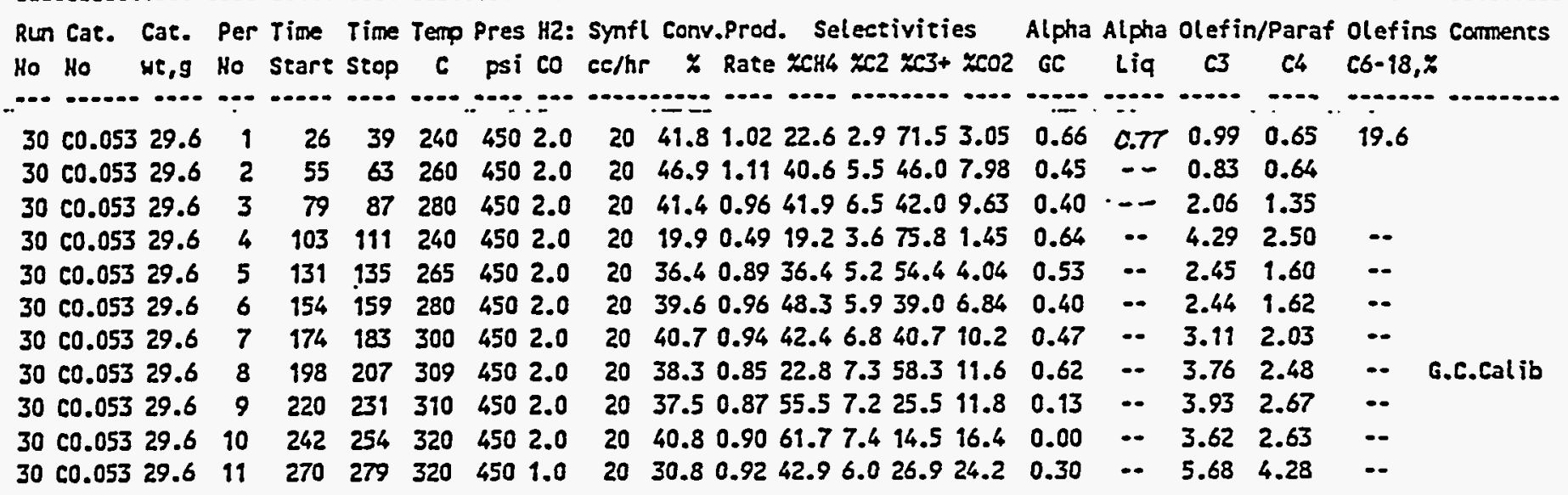


Table 8

SUMAARY OF MA SBCR RUR RESULIS

a) Unless ocherwise noted, total flow is ca.15 h/win. $5 i P$, or $3 \mathrm{~cm} / \mathrm{sec}$ linear gas flow.

b)Conversion is total Co conversion over the period (z).

c)Conversion and selectivities are calculated using $\mathrm{HZ}$ as an interal

standard in the GC analysis of the offgas.

d) Prod. race: Rate for production of total hydrocarbons,C1t (k8/kg cat., hr).

-)Alpha: Based on CC analysis of offgas and liquid product.

Run Cat. Cat. Per Iime Time Iemp Pres 82: Synfl Conv.Pzod. Selectivities Alpha Alpha Olefin/Paraf Olefins Ccoments No to we,8 to start stop C psi CO ee/hr I Rate zCg4 2C2 2C3+ ZCO2 GC Liq C3 C4 C5-18,Z

Ho No

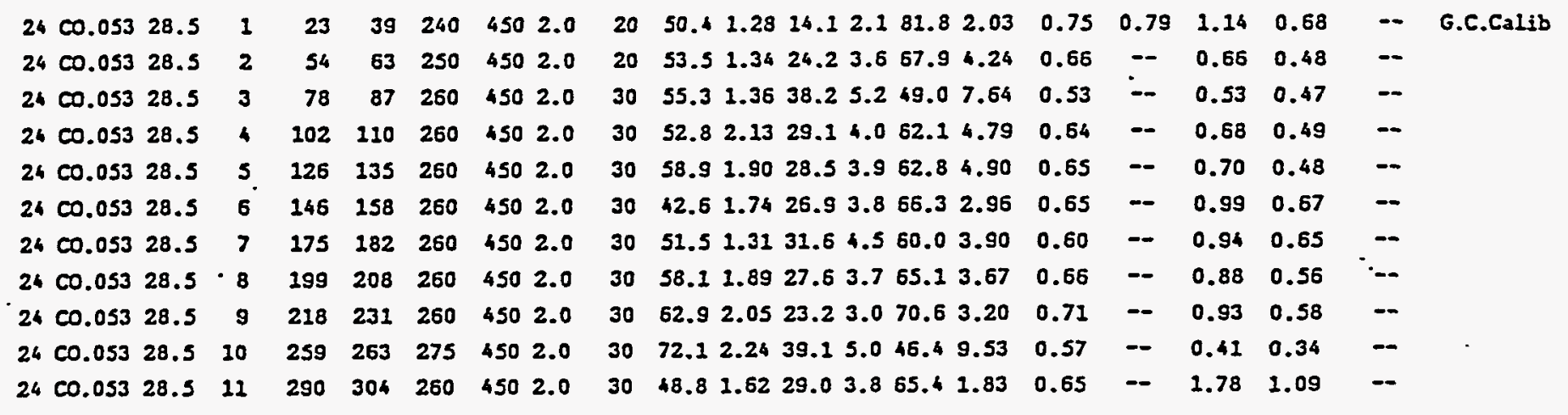

Feed Gas Rates

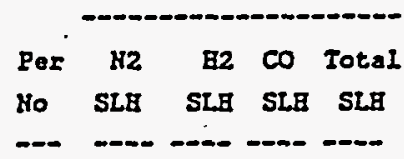

$\begin{array}{rrrrr}1 & 563 & 225 & 112 & 900 \\ 2 & 563 & 225 & 112 & 900 \\ 3 & 563 & 225 & 112 & 900 \\ 4 & 360 & 360 & 180 & 900 \\ 5 & 288 & 288 & 144 & 720 \\ 6 & 540 & 360 & 180 & 1080 \\ 7 & 563 & 225 & 112 & 900 \\ 8 & 200 & 288 & 144 & 632 \\ 9 & 108 & 288 & 144 & 540 \\ 10 & 108 & 288 & 144 & 540 \\ 1 & 108 & 288 & 144 & 540\end{array}$


Table 9

SUMHARY OF MB SBCR RUH RESULTS

a) Unless otherwise noted, total flow is ca.15 L/min. STP, or $3 \mathrm{~cm} / \mathrm{sec}$ linear gas flow.

b) Conversion is total co conversion over the period ( $x$ ).

c) Conversion and selectivities are calculated using $\mathrm{HZ}$ as an internal

standard in the $G C$ analysis of the offgas.

d)Prod. rate: Rate for production of total hydrocarbons, C1+ (kg/kg cat., hr).

e)Alpha: Based on GC analysis of offgas and liquid product.

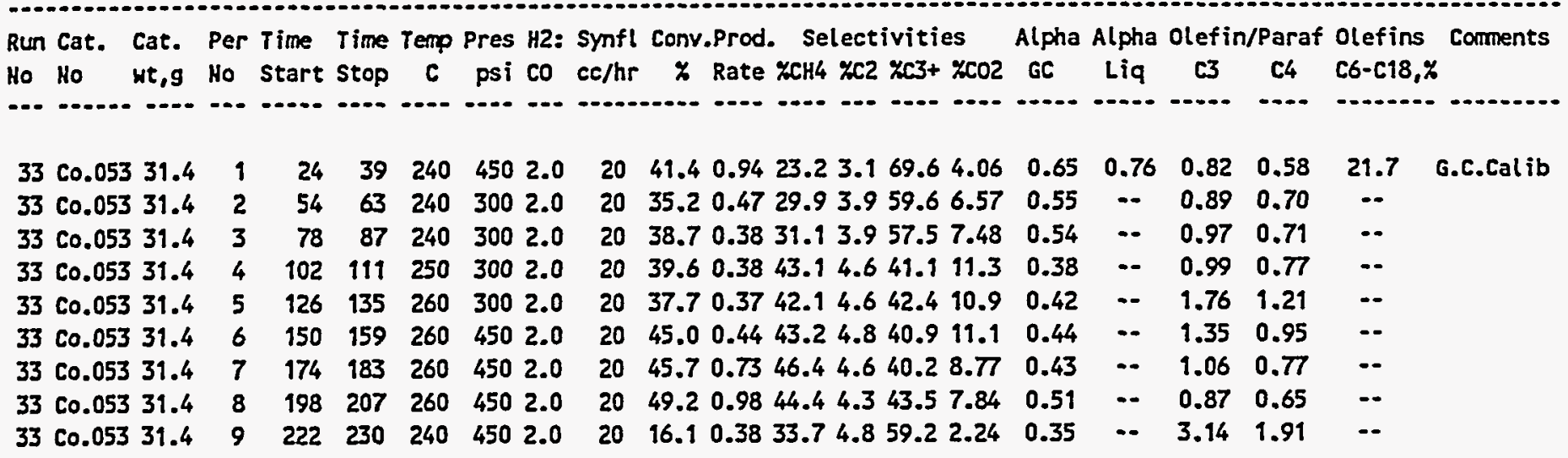

Feed Gas Rates

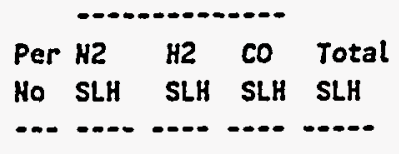

$\begin{array}{rrrrr}1 & 563 & 225 & 112 & 900 \\ 2 & 338 & 135 & 67 & 540 \\ 3 & 250 & 100 & 50 & 400 \\ 4 & 250 & 100 & 50 & 400 \\ 5 & 250 & 100 & 50 & 400 \\ 6 & 250 & 100 & 50 & 400 \\ 7 & 160 & 160 & 80 & 400 \\ 8 & 100 & 200 & 100 & 400 \\ 9 & 563 & 225 & 112 & 900\end{array}$ 
SURAARY OE MA SBCR RUN RESULTS

a) Unless otherwise noted. total flow is ca.15 L/min. SiP. of $3 \mathrm{~cm} / \mathrm{sec}$ linear gas flow.

b) Conversion is total $C O$ conversion over the period $(z)$.

c)Conversion and selectivities are calculated using $t 2$ as an internal

standard in the $G C$ analysis of the offgas.

d) Prod. rate: Rate for production of total hydrocarbons,C1+ (kg/kg cat. .hr).

e)Alpha: Based on GC analysis of offgas and liquid product.

Run Cat. Cat. Per Iime Time Temp Pzes 82: Synfl Conv.Prod. Selectivities Alpha Alpha Olefin/Paraf Olefins Canments

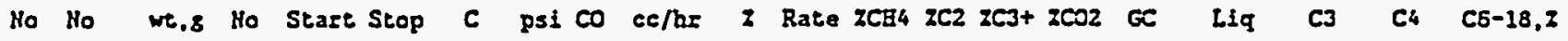

\begin{tabular}{|c|c|c|c|c|c|c|c|c|c|c|c|c|c|c|c|c|c|c|}
\hline AL. 10 & 5.6 & 1 & 23 & 39 & 240 & 450 & 2.0 & 20 & 21.9 & 1.01 & 5.4 & 591 & .25 & 0.79 & 0.85 & 3.60 & 2.27 & 25.9 \\
\hline & 15.6 & 2 & 54 & 63 & 240 & 450 & 0 & 20 & 21.7 & .30 & 8.3 & 189.9 & .10 & 0.80 & - & 3.78 & 2.45 & -- \\
\hline & & 3 & 78 & 87 & 240 & 450 & .0 & 20 & 17.2 & .04 & 11.7 & 36.1 & 1.39 & 0.76 & - & 3.57 & 2.30 & 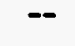 \\
\hline & & & 102 & 211 & 240 & 450 &. .0 & 0 & 17.7 & .01 & 11.9 & 36.0 & 1.40 & 0.77 & -- & 3.52 & 2.28 & - \\
\hline & & & 118 & 126 & 240 & 450 & 2.0 & 20 & 16.2 & 0.75 & 4.9 & 1.792 .3 & 1.10 & 0.77 & -- & 4.29 & 2.75 & \\
\hline
\end{tabular}

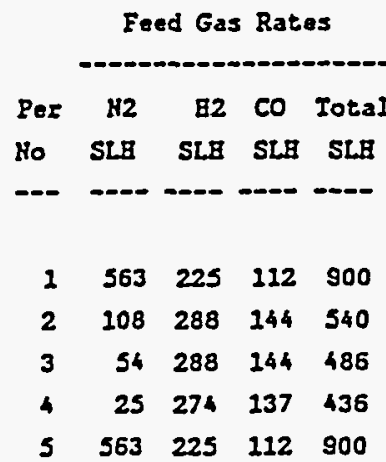


Table 11

DATE: $12 / 30 / 94$

COMPARISON OF CONVERSION AND SEIECTIVITY OF

CAISICAT PRODUCED CATAIYSTS AT STARTUP CONDITIONS

\begin{tabular}{cccc} 
Period No. & Temp. & Pres. & H2/CO Ratio \\
\hline$I$ & $240 \mathrm{C}$ & $450 \mathrm{psi}$ & -2.0
\end{tabular}

a) Total flow is ca. $15 \mathrm{~L} / \mathrm{min}$. STP, or $3 \mathrm{~cm} / \mathrm{sec}$ Iinear gas flow.

Flows: N2-563 SLH, H2-225 SIH, CO-112.5 SIH

b) Conversion is total co conversion over the period ( $q)$.

c) Conversion and selectivities are calculated using N2 as an internal

standard in the GC analysis of the offgas.

d) Prod. rate: Rate for production of total hydrocarbons clt (kg/kg cat., hr). e) Alpha: Based on GC analysis of offgas and Iiquid product.

\begin{tabular}{|c|c|c|c|c|c|c|c|c|c|c|c|c|c|}
\hline $\begin{array}{l}\text { Run } \\
\text { No }\end{array}$ & $\begin{array}{l}\text { Cat. } \\
\text { No }\end{array}$ & $\begin{array}{l}\text { Cat. } \\
\text { wt, g }\end{array}$ & $\begin{array}{c}\text { Conv } \\
\frac{8}{8}\end{array}$ & $\begin{array}{l}\text { Prod. } \\
\text { Rate }\end{array}$ & $\begin{array}{r}\mathrm{Se} \\
8 \mathrm{CH} 4\end{array}$ & $\begin{array}{l}e c t \\
8 c 2\end{array}$ & $\begin{array}{l}\text { iviti } \\
\text { \&c3t }\end{array}$ & $\begin{array}{l}25 \\
8 \mathrm{CO} 2\end{array}$ & $\begin{array}{l}\text { Alpha } \\
\text { GC }\end{array}$ & $\begin{array}{l}\text { Alpha } \\
\text { Iiq }\end{array}$ & $\begin{array}{l}\text { Cataly } \\
\text { Proms }\end{array}$ & $\begin{array}{l}\text { st Compo } \\
\text { Metalo }\end{array}$ & $\begin{array}{l}\text { sit. } \\
\text { supp }\end{array}$ \\
\hline-4 & SBCR & & & & & & & & & & & & \\
\hline $\begin{array}{l}10 \\
11\end{array}$ & $\begin{array}{l}\text { CAL. } 02 \\
\text { CAL. } 03\end{array}$ & $\begin{array}{l}15.9 \\
15.6\end{array}$ & $\begin{array}{l}34.5 \\
29.7\end{array}$ & $\begin{array}{l}1.58 \\
1.39\end{array}$ & $\begin{array}{l}12.9 \\
13.5\end{array}$ & $\begin{array}{l}.0 \\
.1\end{array}$ & $\begin{array}{l}83.9 \\
83.4\end{array}$ & $\begin{array}{l}1.23 \\
1.08\end{array}$ & $\begin{array}{l}0.75 \\
0.74\end{array}$ & $\begin{array}{l}0.81 \\
0.86\end{array}$ & $\begin{array}{l}0.0 \\
0.0\end{array}$ & $\begin{array}{l}0.5 R u \\
0.5 R u\end{array}$ & Al \\
\hline $\begin{array}{l}16 \\
17 \\
18 \\
19 \\
20 \\
23 \\
26\end{array}$ & $\begin{array}{l}\text { CAI.04 } \\
\text { CAI.08 } \\
\text { CAI.07 } \\
\text { CAI.05 } \\
\text { CAI.06 } \\
\text { CAI.09 } \\
\text { CAL.10 }\end{array}$ & $\begin{array}{l}16.1 \\
15.0 \\
15.0 \\
15.7 \\
15.0 \\
15.0 \\
15.6\end{array}$ & $\begin{array}{r}26.3 \\
16.9 \\
20.6 \\
26.6 \\
5.6 \\
17.4 \\
21.9\end{array}$ & $\begin{array}{l}1.34 \\
0.93 \\
1.03 \\
1.22 \\
0.26 \\
0.84 \\
1.01\end{array}$ & $\begin{array}{l}7.1 \\
5.9 \\
7.7 \\
7.3 \\
3.1 \\
0.1 \\
5.4\end{array}$ & $\begin{array}{l}1.3 \\
0.8 \\
1.2 \\
1.3 \\
2.1 \\
1.2 \\
1.5\end{array}$ & $\begin{array}{l}90.3 \\
91.7 \\
90.0 \\
90.2 \\
92.0 \\
97.9 \\
91.9\end{array}$ & $\begin{array}{l}1.38 \\
1.36 \\
1.09 \\
1.19 \\
2.86 \\
0.95 \\
1.25\end{array}$ & $\begin{array}{l}0.80 \\
0.82 \\
0.81 \\
0.82 \\
0.80 \\
0.83 \\
0.79\end{array}$ & $\begin{array}{l}0.85 \\
0.88 \\
0.88 \\
0.86 \\
0.90 \\
0.88 \\
0.85\end{array}$ & $\begin{array}{l}0.3 \mathrm{~K} \\
0.3 \mathrm{~K} \\
0.3 \mathrm{~K} \\
0.3 \mathrm{~K} \\
0.3 \mathrm{~K} \\
0.3 \mathrm{~K} \\
0.3 \mathrm{~K}\end{array}$ & $\begin{array}{l}0.5 R u \\
0.5 R u \\
0.5 R u \\
0.5 R u \\
0.5 R u \\
0.5 R u \\
0.5 R u\end{array}$ & \\
\hline
\end{tabular}

$\mathrm{M}-3 \mathrm{SBCR}$

$\begin{array}{llllllllllllll}12 & C 0.018 & 15.6 & 33.8 & 1.56 & 9.7 & 2.0 & 86.6 & 1.68 & 0.75 & 0.85 & 0.0 & 0.5 \mathrm{Ru} & \mathrm{AI} \\ 23 & \mathrm{C} 0.047 & 15.8 & 28.2 & 1.29 & 7.7 & 1.5 & 89.3 & 1.47 & 0.79 & 0.84 & 0.3 \mathrm{~K} & 0.5 \mathrm{Ru} & \mathrm{Al}\end{array}$

Note: Catalysts for all runs except Runs $17,18, \& 20$ screened

thru $150 \times 400$ mesh.

Catalyst CAL.04 was calcined in air; Catalyst CAL.05 processed in N2.

Cat. No. CAL.06 was H2 reduced and air stabilized before charging.

Cat. Nos. CAL.08 and CAL.07 were prereduced and wax coated. CAL.08 was calcined in $\mathrm{N} 2$; CAL.07 was reduced in $\mathrm{H} 2$ without prior calcination. $\mathrm{H} 2$ to $C O$ ratio for Runs 16 and 17 was $1.76 / 1$.

Cat. Nos. Co.018 \& Co.047 were prepared by Pitt. Shown for comparison.

Cat. No. CAL.09 was prereduced and coated with soya.

Cat. No. CAI.IO was prepared on Condea Alumina. 
Table 12

DATE: $12 / 30 / 94$

COMPARISON OF CONVERSION AND SELECTIVITY OF

METHANE REDUCTION CATALYSTS AT STARTUP CONDITIONS

\begin{tabular}{cccc} 
Period No. & Temp. & Pres. & H2/CO Ratio \\
\hline$I$ & $240 \mathrm{C}$ & $450 \mathrm{psi}$ & 2.0
\end{tabular}

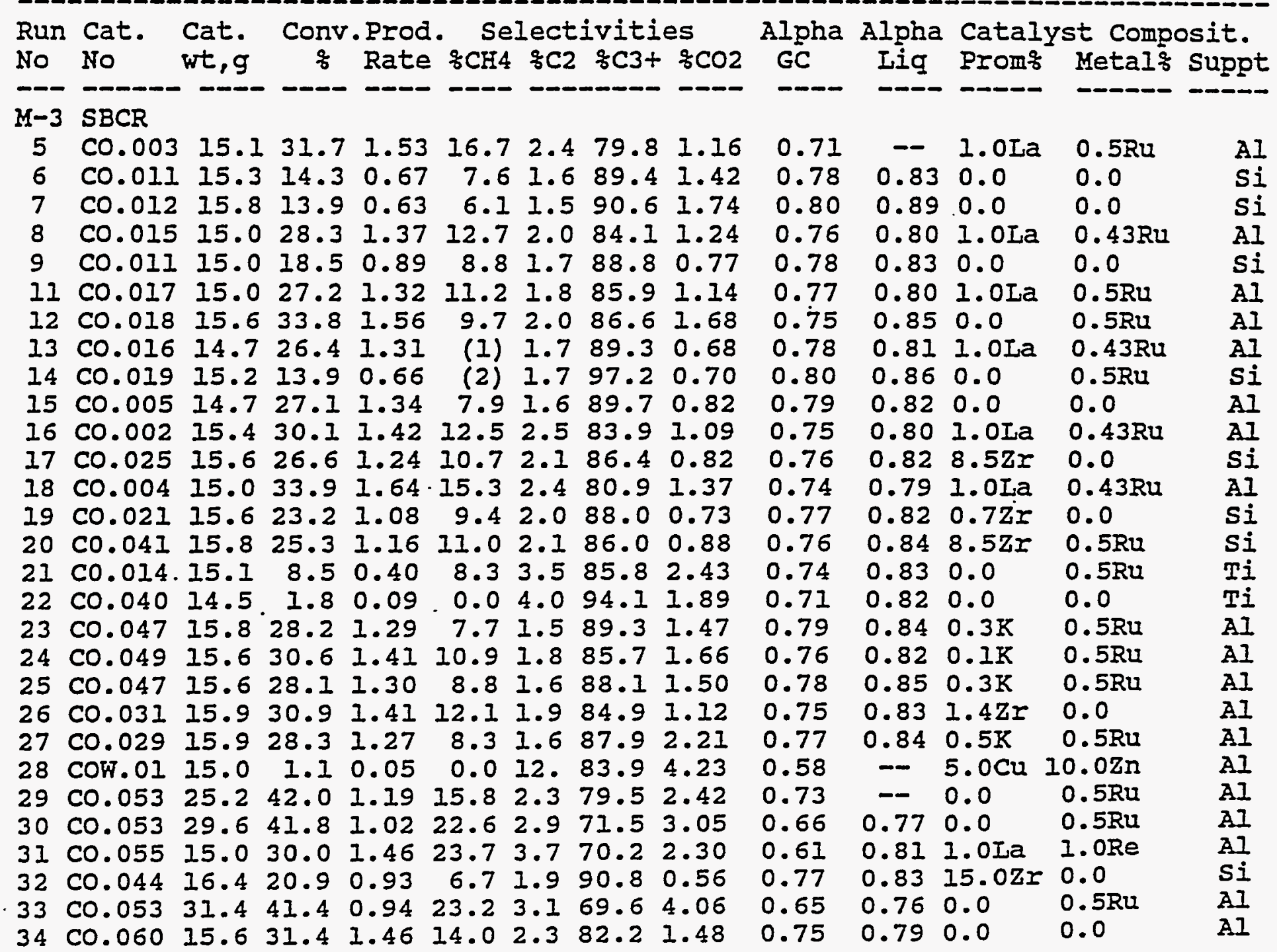

Note: Catalyst Run 9 screened thru $170 \times 400$ mesh. Catalysts For Runs 11 through 28 (except Runs 21 and 22) screened thru $150 \times 400$ mesh. Catalysts for Runs 21 and 22 screened thru $100 \times 400$ mesh. Catalysts Nos. Co.029 and Co.060 contained 30 wto cobalt.

G.C.Problems

(1) Weak TCD filaments in G.C. CH4 peaks smaller than expected.

(2) Internal valve in G.C. leaked. CH4 peak undetected. 


\section{SBCR CATALYST EXTRACTIONS}

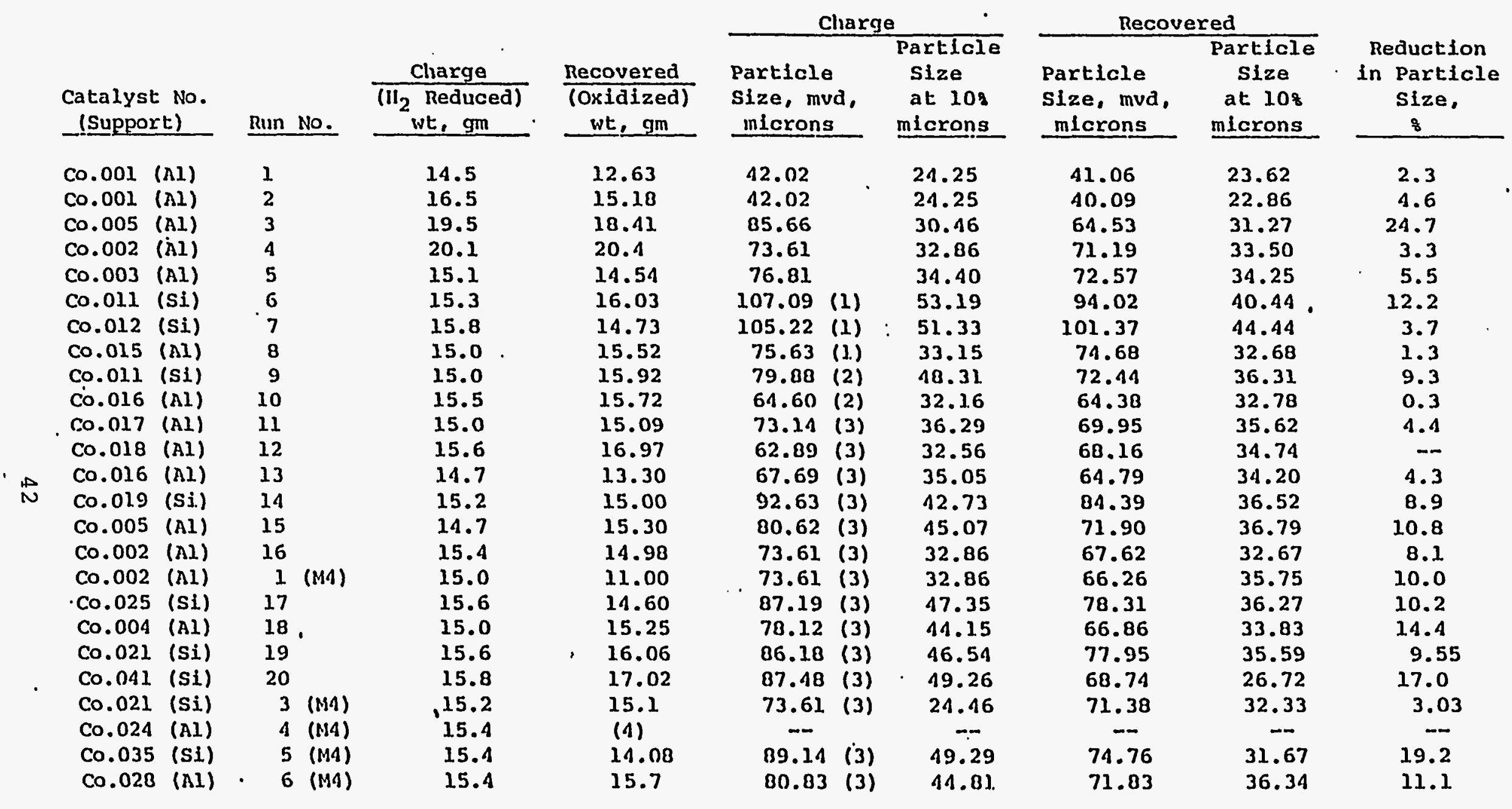

(1) Screened through $100 \times 400$ mesh screens.

(2) Screened through $170 \times 100$ mesh screens.

(3) Screened through $150 \times 400$ mesh screens.

(1) Did not Ej.lter, too waxy

Note: The charge weights are In the hydrogen-reduced gtate and the recovered welghts are in the oxidized state. The particle size is reported as the mean volume dianeter (mud) as measured by a Microtrac particle slze analyzer. 
Table 13

(Continued)

SBCR CATALYST EXTRACTIONS

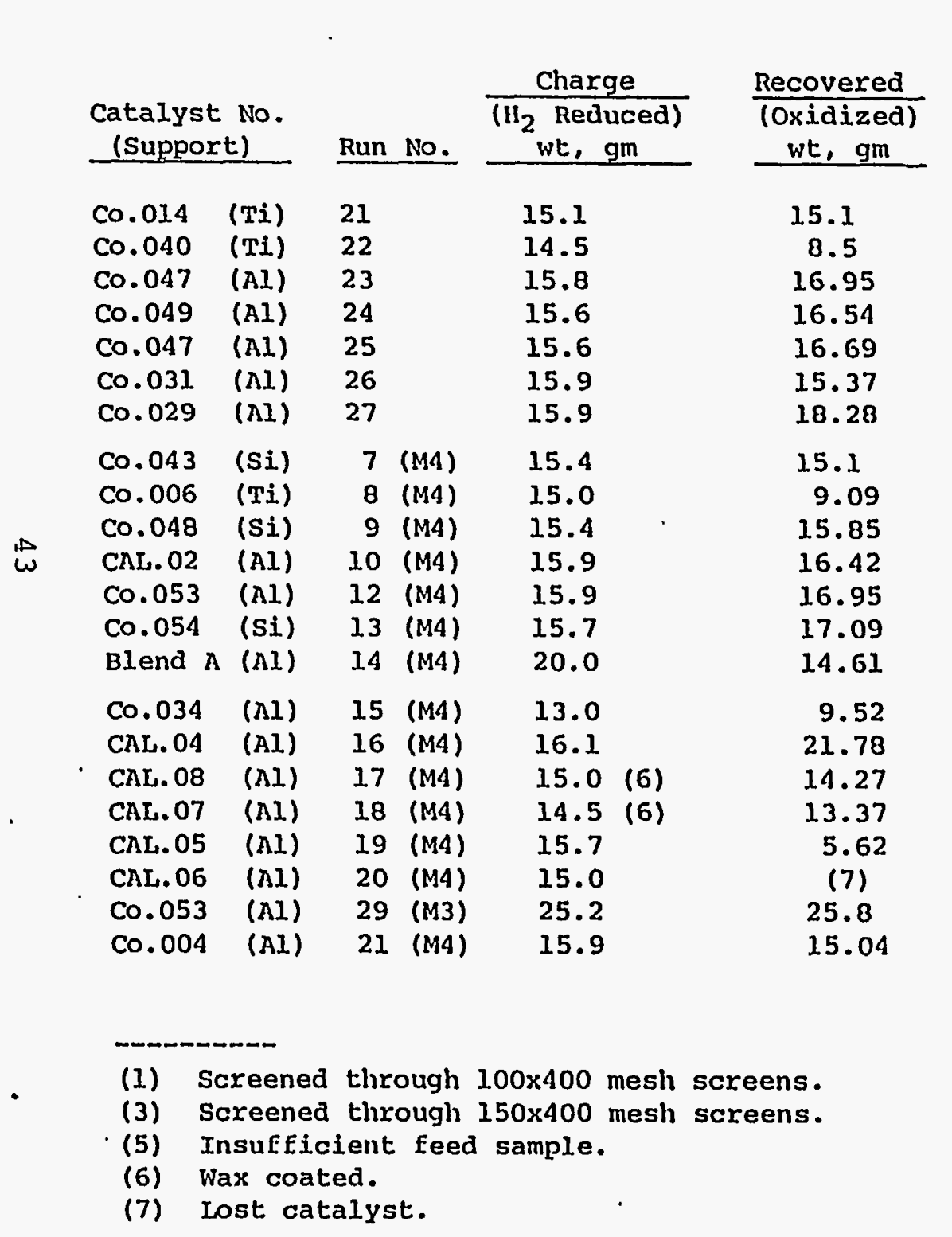

\begin{tabular}{lc}
$\frac{\text { Charge }}{\text { Particle }}$ \\
Particle & Size \\
Size, mvd, at loz \\
microns \\
\hline
\end{tabular}

111.70 (1)

(5)

83.42 (3)

81.3 (3)

79.36 (3)

75.48 (3)

76.39 (3)

(5)

$121.38 \quad(1)$

82.40 (3)

74.40 (3)

82.02 (3)

101.17 (3)

86.23 (3)

\subsection{5}

(5)

44.41

42.39

41.72

38.34

39.56

(5)

52.80

40.94

37.79

43.10

57.54

(5)

71.54 (3)

46.36

$-$

72.75

72.62

75.63

81.38
(5)

36.28

25.60

--

36.90

37.33

40.80

41.82
Recovered

\begin{tabular}{cc}
\hline & Particle \\
Particle & Size \\
Size, mud. & at 108 \\
microns & microns \\
\hline
\end{tabular}

106.66

130.40

76.46

70.11

69.09

68.42

68.72

85.51

99.31

70.69

65.89

69.53

82.37

77.43

88.26

66.50

62.80

67.02

71.62

$-$

71.09

33.49
51.40
39.02
35.87
36.16
35.39
36.04
40.20
38.62
25.53
34.14
35.76
37.89
42.81
45.28
32.82
26.03
28.12
36.55
--
38.20
37.61

Reduction

in Particle

Size, 8

4.51
--
8.34
13.8
12.94
9.35
10.04
--
18.2
14.2
11.44
15.23
18.58
10.21
--
7.05
0.51
--
1.55
--
6.00
10.44


Table 13

(Continued)

SBCR CATALYST EXTRACTIONS

\begin{tabular}{|c|c|c|c|c|c|c|c|c|}
\hline \multirow[b]{3}{*}{$\begin{array}{l}\text { Catalyst No. } \\
\text { (Support) }\end{array}$} & \multirow[b]{3}{*}{ Run No. } & & \multirow[b]{3}{*}{$\begin{array}{c}\text { Recovered } \\
\text { (Oxidized) } \\
\text { wt, gm }\end{array}$} & \multicolumn{2}{|c|}{ Charge } & \multicolumn{2}{|c|}{ Recovered } & \multirow{3}{*}{$\begin{array}{c}\text { Reduction } \\
\text { in Particle } \\
\text { Size, } \\
8 \\
\end{array}$} \\
\hline & & & & & Particle & & Particle & \\
\hline & & $\begin{array}{c}\frac{\text { Charge }}{\left(\mathrm{H}_{2} \text { Reduced }\right)} \\
\text { wt, gm }\end{array}$ & & $\begin{array}{c}\text { Particle } \\
\text { Size, mvd, } \\
\text { microns } \\
\end{array}$ & $\begin{array}{c}\text { Size } \\
\text { at } 108 \\
\text { microns } \\
\end{array}$ & $\begin{array}{c}\text { Particle } \\
\text { Size, mud, } \\
\text { microns }\end{array}$ & $\begin{array}{c}\text { Size } \\
\text { at 10z } \\
\text { microns } \\
\end{array}$ & \\
\hline Blend B (Al) & 22 (M4) & 30.5 & 31.75 & 77.85 & 43.10 & 73.74 & 38.80 & 5.28 \\
\hline CAL.09 (AI) & 23 (M4) & $30.0(6)$ & 14.34 & 63.12 & 25.60 & 62.10 & 26.32 & 1.62 \\
\hline Co.053-3 (Al) & 24 (M4) & 28.5 & 27.85 & 75.63 & 40.80 & 70.69 & 35.03 & 6.53 \\
\hline Co.055 (AI) & 31 (M3) & 15.0 & 14.67 & 85.58 & 46.27 & 74.56 & 39.00 & 12.88 \\
\hline $\mathrm{Co.056}$ (Si) & 25 (M4) & $i 5.0$ & 14.93 & 99.93 & 54.62 & 88.48 & 43.26 & 11.46 \\
\hline Co.044 (Si) & 32 (M3) & 16.4 & 17.01 & 92.15 & 49.30 & 84.92 & 40.21 & 7.85 \\
\hline CAL.10 (AI) & 26 (M4) & 15.6 & 14.63 & 83.05 & 47.11 & 81.12 & 45.87 & 2.32 \\
\hline Co.053-4 (AI) & 33 (M4) & 31.4 & 31.9 & 84.09 & 44.40 & 73.50 & 37.10 & 12.6 \\
\hline Co.050 (AI) & 27 (M4) & 14.6 & 10.62 & (5) & $(5)$ & 91.37 & $4 \ddot{4} .07$ & -- \\
\hline $\mathrm{Co.060} \mathrm{(Al)}$ & 34 (M3) & 15.6 & 15.8 & 87.45 & 47.08 & 80.89 & 43.42 & 7.50 \\
\hline
\end{tabular}

(5) Insufficient feed sample. 


\section{APPENDIX A}

Co Catalyst Formulations 


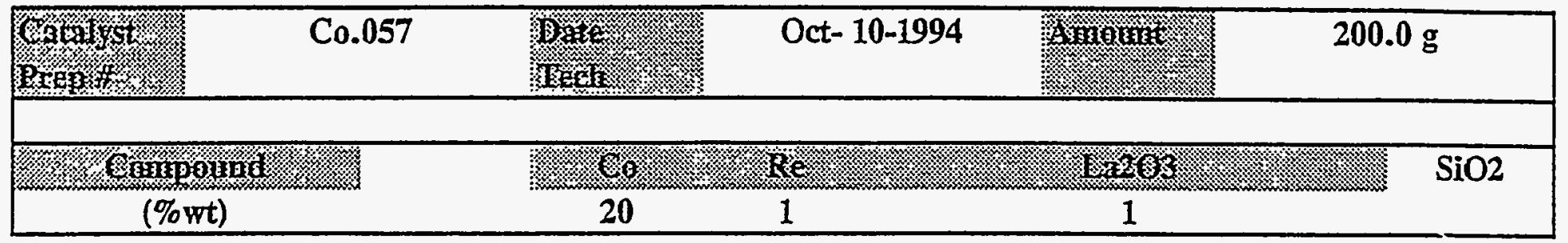

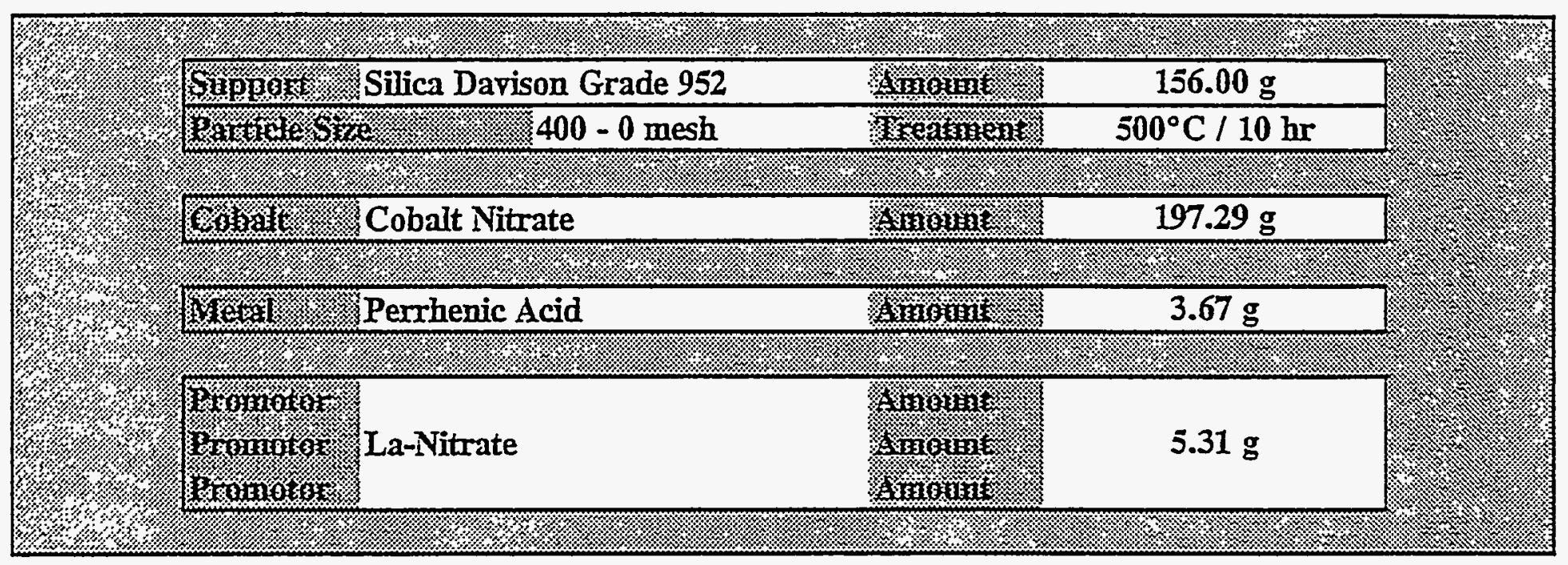

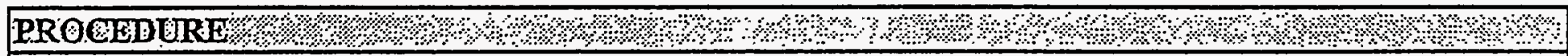 Preparaton \% \%: $\quad \mathrm{X}$ Incipient Wetuess Ion Exchange \\ Wet Impregnation \\ Other}

Notes. : Incipient wetness: aqueous solution - ca. $1.2 \mathrm{ml} / \mathrm{g}$ support

Dry catalyst precursor in an oven at $115^{\circ} \mathrm{C} / 5 \mathrm{hr}$

Calcimation

Notes

Temperature

$50 \mathrm{~g}$ of the catalyst precursor is not to be calcined !! - 
Co.057: $\quad 20 w+\%$ Co

$1 \mathrm{wt} \% \operatorname{Re}$

$1 \mathrm{wt} \% \mathrm{La}_{2} \mathrm{O}_{3}$

Silica

$\mathrm{La} / \mathrm{Re}$-promoted $\mathrm{SiO}_{2}$-supported catalyst comparable to $\mathrm{Co} .055$ where $\mathrm{SiO}_{2}$ is used as support instead of $\mathrm{Al}_{2} \mathrm{O}_{3}$

\section{Preparation Procedure:}

Calcine the $\mathrm{SiO}_{2}$ at $500^{\circ} \mathrm{C}$ for $10 \mathrm{hrs}$. Presieve to $>38$ microns (400-0 mesh).

Impregnate the support with an aqueous solution of Co nitrate $\left[\mathrm{Co}\left(\mathrm{NO}_{3}\right)_{2} \cdot \mathrm{H}_{2} \mathrm{O}\right.$ ], perrhenic acid [HReO $\mathrm{H}_{4}$, lanthanum nitrate, and using an appropriate quantity to get incipient wemess (ca. $1.2 \mathrm{ml} / \mathrm{g}$ ) with the desired loading of $\mathrm{Co}$.

Dry the catalyst precursor in an oven for 5 hrs at $115^{\circ} \mathrm{C}$ with moderate stirring.

The dried catalyst is then calcined in air by raising its temperature at a heating rate of ca. $1^{\circ} \mathrm{C} / \mathrm{min}$ to $300^{\circ} \mathrm{C}$ and holding for $2 \mathrm{hrs}$.

Reduction Procedure before Reaction:

Reduce the catalyst in a pure hydrogen flow of $3000 \mathrm{cc} / \mathrm{g} / \mathrm{hr}$ by heating at $1^{\circ} \mathrm{C} / \mathrm{min}$ to $250^{\circ} \mathrm{C}$ and holding for $10 \mathrm{hrs}$. 


\begin{tabular}{|c|c|c|c|c|}
\hline 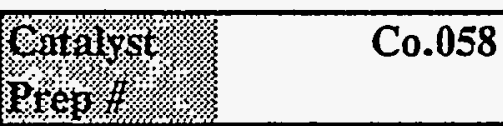 & Mre & Oct. 10-1994 & A miginit & $200.0 \mathrm{~g}$ \\
\hline 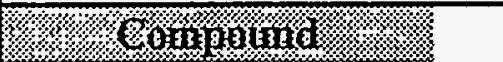 & !ে - & & 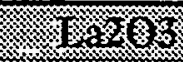 & $\overline{\mathrm{Al} 2 \mathrm{O3}}$ \\
\hline (\%wt) & 20 & & 1 & \\
\hline
\end{tabular}

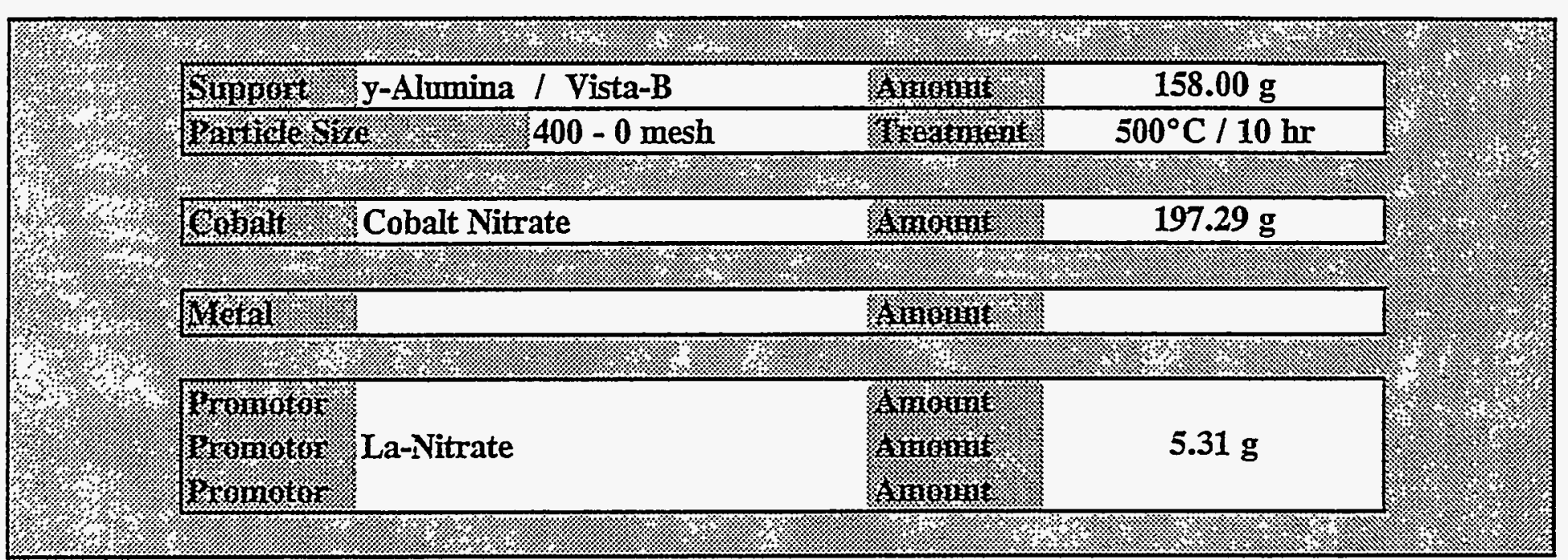

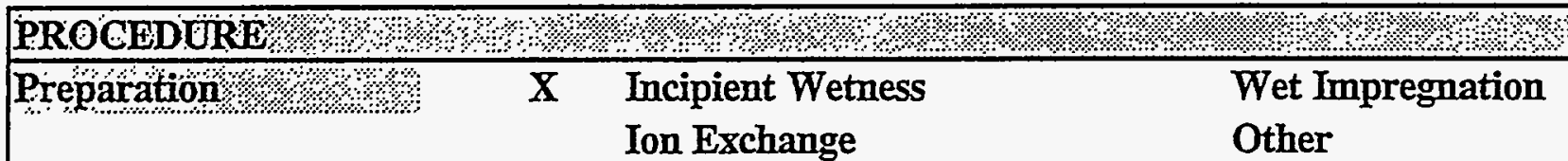

Notes / Incipient wetness: aqueous solution - ca. $1.2 \mathrm{ml} / \mathrm{g}$ support

Dry catalyst precursor in an oven at $115^{\circ} \mathrm{C} / 5 \mathrm{br}$

Calcination 12 Temperature $300^{\circ} \mathrm{C}$ Time $2 \mathrm{hr}$

Notes . $50 \mathrm{~g}$ of the catalyst precursor is not to be calcined !! 
Co.058: $\quad 20 \mathrm{wt} \%$ Co

$1 \mathrm{wt} \% \mathrm{La}_{2} \mathrm{O}_{3}$

$\gamma$-alumina

La-promoted $\mathrm{Al}_{2} \mathrm{O}_{3}$-supported catalyst comparable to Co.055 without Re.

Preparation Procedure:

Calcine the $\gamma$-alumina at $500^{\circ} \mathrm{C}$ for $10 \mathrm{hrs}$. Use Vista B alumina. Presieve to $38-63$ microns (400-250 mesh).

Impregnate the support with an aqueous solution of $\mathrm{Co}$ nitrate $\left[\mathrm{Co}\left(\mathrm{NO}_{3}\right)_{2} \cdot 6 \mathrm{H}_{2} \mathrm{O}\right]$, lanthanum nitrate, and using an appropriate quantity to get incipient wetness (ca. $1.2 \mathrm{ml} / \mathrm{g}$ ) with the desired loading of Co.

Dry the catalyst precursor in an oven for 5 hrs at $115^{\circ} \mathrm{C}$ with moderate stirring.

The dried catalyst is then calcined in air by raising its temperature at a heating rate of ca. $1^{\circ} \mathrm{C} / \mathrm{min}$ to $300^{\circ} \mathrm{C}$ and holding for $2 \mathrm{hrs}$.

Reduction Procedure before Reaction:

Reduce the catalyst in a pure hydrogen flow of $3000 \mathrm{cc} / \mathrm{g} / \mathrm{hr}$ by heating at $1^{\circ} \mathrm{C} / \mathrm{min}$ to $350^{\circ} \mathrm{C}$ and holding for $10 \mathrm{hrs}$. 


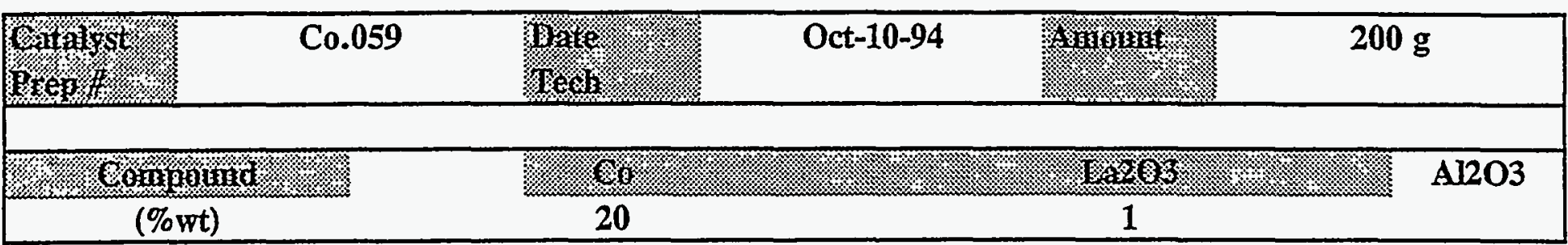

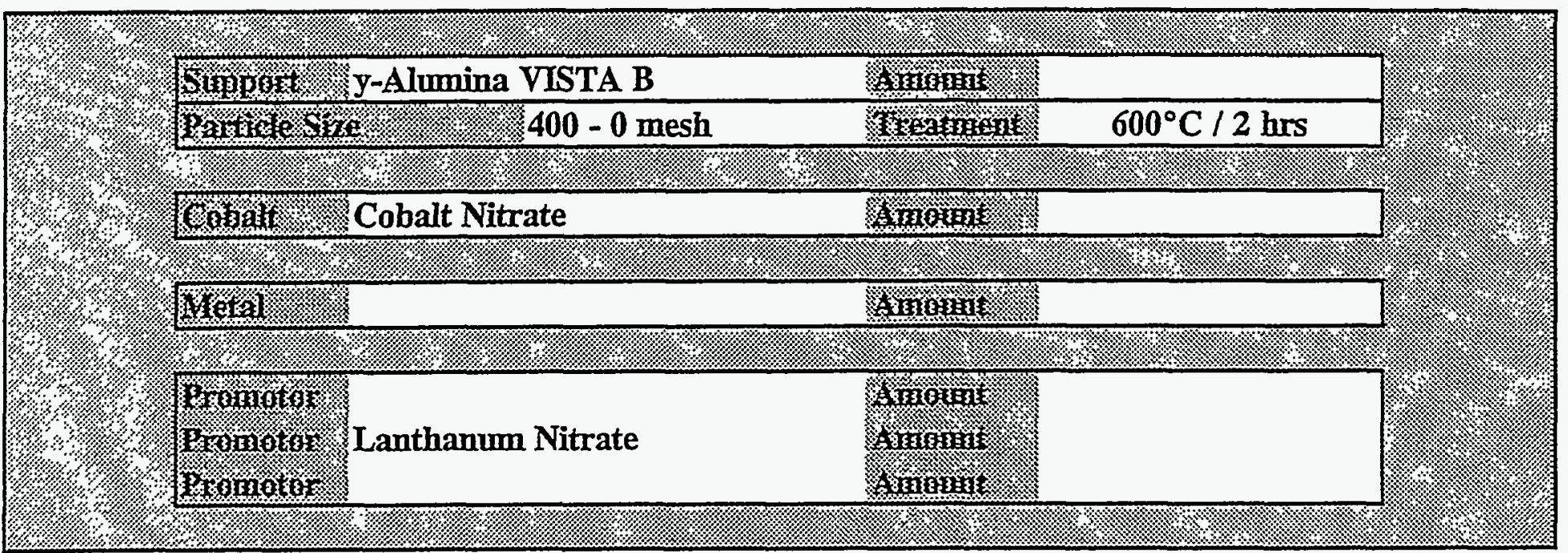

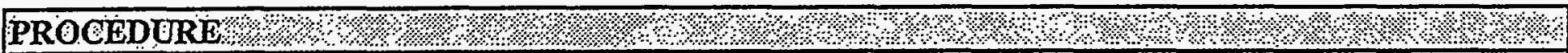
Preparafion

Ion Exchange

Wet Impregnation

Other

Notes / /ncipient wetness: aqueous solution of cobalt nitrate - ca. $1 \mathrm{ml} / \mathrm{g}$

Dry catalyst precursor at $120^{\circ} \mathrm{C} / 16 \mathrm{hrs}$

Incipient wetness: aqueous solution of $\mathrm{La}$

Calcination $2 . \quad \underline{300 \operatorname{deg} C}$ Time $\underline{2 ~ h r}$

Notes : $50 \mathrm{~g}$ of precursor is not to be calcined 
Co.059: $\quad 20 \mathrm{wt} \%$ Co

$1 \mathrm{wt} \% \mathrm{La}_{2} \mathrm{O}_{3}$

$\gamma$-alumina

Similar to Co.015, but without Ru.

\section{Preparation Procedure:}

Calcine the $\gamma$-alumina at $600^{\circ} \mathrm{C}$ for 2 hrs. Use Vista B alumina. Presieve to $>38$ microns (400-0 mesh).

Impregnate the support with an aqueous solution of $\mathrm{Co}$ nitrate $\left[\mathrm{Co}\left(\mathrm{NO}_{3}\right)_{2} \cdot 6 \mathrm{H}_{2} \mathrm{O}\right]$ using an appropriate quantity to get incipient wetness (ca. $1 \mathrm{ml} / \mathrm{g}$ ) with the desired loading of Co.

Dry the catalyst precursor for 16 hrs at $120^{\circ} \mathrm{C}$.

Impregnate the catalyst precursor with an aqueous solution of lanthanum nitrate hexahydrate using an appropriate quantity to get incipient wetness with the desired loading of $\mathrm{La}_{2} \mathrm{O}_{3}$.

Dry the catalyst precursor at $90^{\circ} \mathrm{C}$ with moderate stirring.

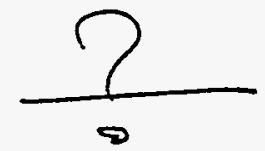

Reduction Procedure before Reaction:

Reduce the catalyst in a pure hydrogen flow of $1000 \mathrm{cc} / \mathrm{g} / \mathrm{hr}$ by heating to $350^{\circ} \mathrm{C}$ and holding overnight (for $16 \mathrm{hrs}$ ). 


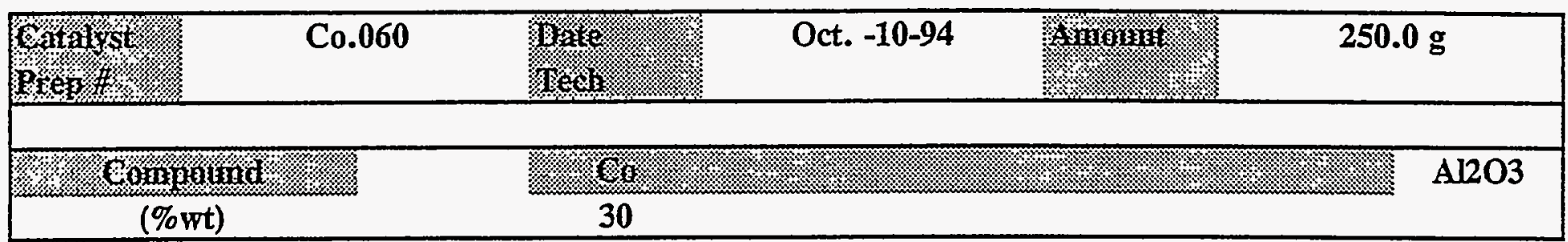

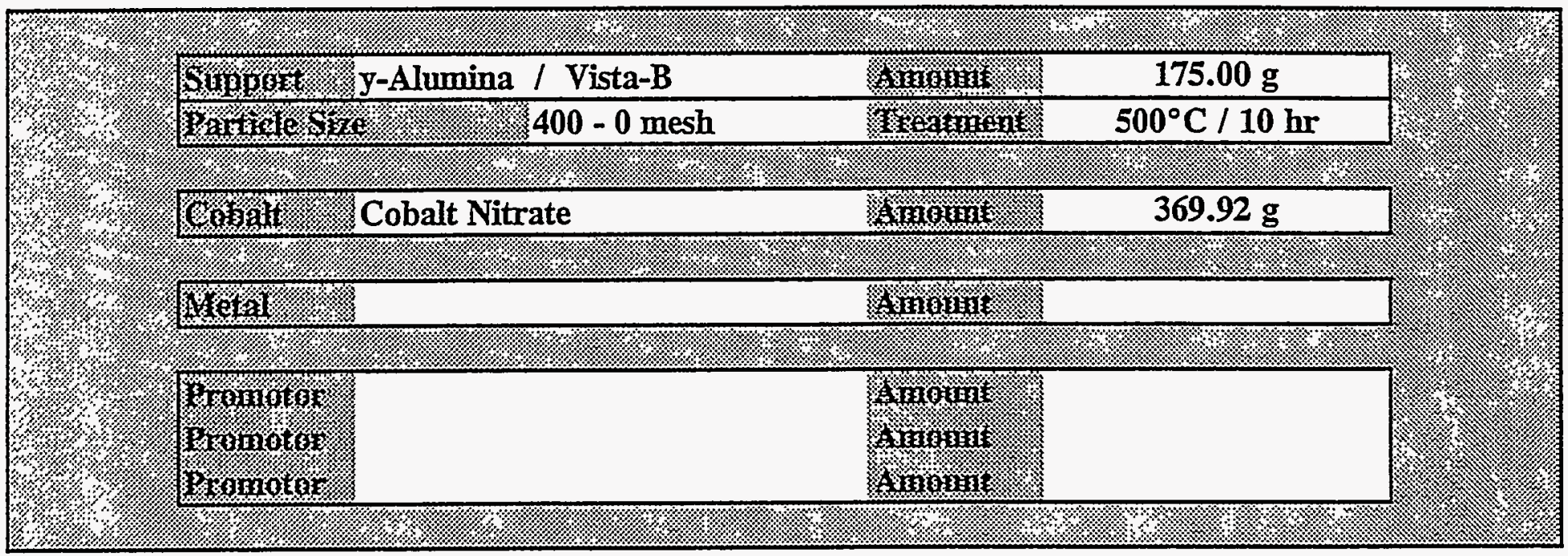

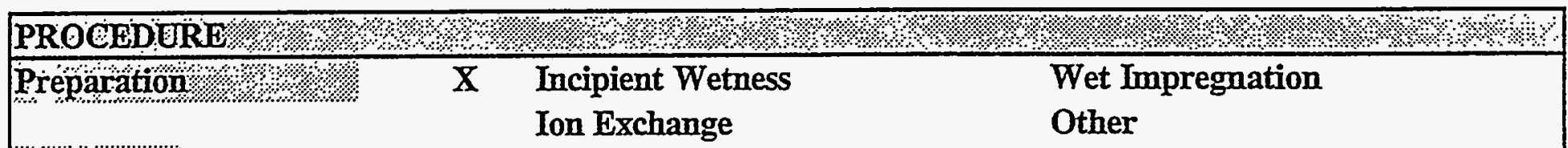

Notes . Incipient wetness: aqueous solution - ca. $1.2 \mathrm{ml} / \mathrm{g}-60 \%$ of loading in first step

Dry catalyst precursor in an oven at $115^{\circ} \mathrm{C} / 12 \mathrm{hrs}$ - calcine $300^{\circ} \mathrm{C} / 2 \mathrm{brs}$

Incipient wetness: aqueous solution - ca. $1.2 \mathrm{ml} / \mathrm{g}$ - remaining $40 \%$ of loading

Repeat drying and calcination

Calcination. Temperature

Time

Notes: 
$30 \mathrm{wt} \% \mathrm{Co}_{0}$

y-alumina

Non-promoted catalyst similar to Co.005, with $30 \%$ Co.

Preparation Procedure:

Calcine the $\gamma$-alumina at $500^{\circ} \mathrm{C}$ for 10 hrs. Use Vista $B$ alumina. Presieve to $>\hat{\jmath} 8$ microns (400-0 mesh).

Impregnate the support in 2 steps with an aqueous solution of Co nitrate $\left[\mathrm{Co}\left(\mathrm{NO}_{3}\right)_{2} \cdot 6 \mathrm{H}_{2} \mathrm{O}\right]$, using an appropriate quantity to get incipient wetmess (ca. 1.2 $\mathrm{ml} / \mathrm{g}$ ) with $60 \%$ of the desired loading of $\mathrm{Co}$ in the first step.

Dry in air at $115^{\circ} \mathrm{C}$ for 12 hours and calcine in air at $300^{\circ} \mathrm{C}$ for 2 hours (heating rate of ca. $1^{\circ} \mathrm{C} / \mathrm{min}$ to $300^{\circ} \mathrm{C}$ ).

In the second step, impregnate the remaining $40 \%$ of the Co. Repeat the drying and calcination procedures.

Reduction Procedure before Reaction:

Reduce the catalyst in a pure hydrogen flow of $3000 \mathrm{cc} / \mathrm{g} / \mathrm{hr}$ by heating at $1^{\circ} \mathrm{C} / \mathrm{min}$ to $350^{\circ} \mathrm{C}$ and holding for $10 \mathrm{hrs}$. 


\begin{tabular}{|c|c|c|c|c|c|}
\hline Caralysi & Co.061 & Mrate & Oct-10-94 & Minnmi & $250.0 \mathrm{~g}$ \\
\hline (T) & & 3 & Rur: & x & $\mathrm{Al203}$ \\
\hline (\% wt) & & 30 & 0.5 & & \\
\hline
\end{tabular}

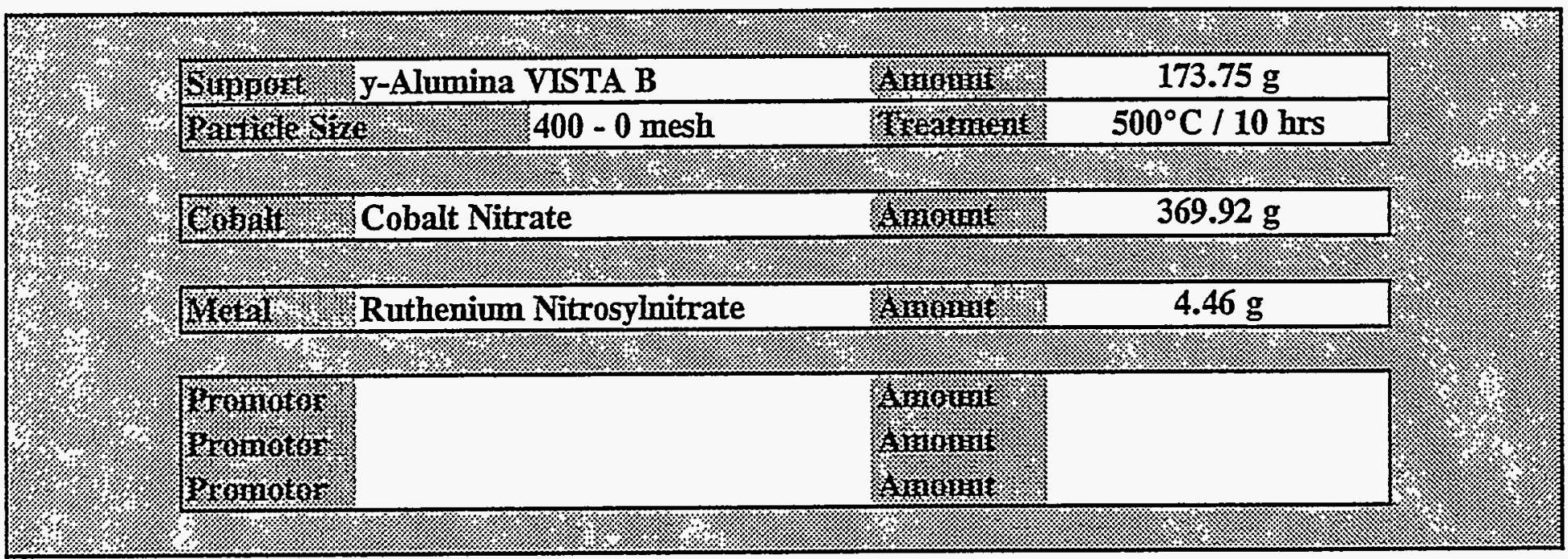

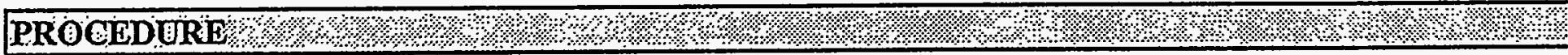
Preparation

$$
\begin{aligned}
& \text { Incipient Wetness } \\
& \text { Ion Exchange }
\end{aligned}
$$

Wet Impregnation

Other

Nótes . Incipient wetness: aqueous solution - ca. $1.2 \mathrm{ml} / \mathrm{g}-60 \%$ of loading in first step

Dry catalyst precursor in an oven at $115^{\circ} \mathrm{C} / 12 \mathrm{hrs}$ - calcine $300^{\circ} \mathrm{C} / 2 \mathrm{hrs}$

Incipient wetness: aqueous solution - ca. $1.2 \mathrm{ml} / \mathrm{g}$ - remaining $40 \%$ of loading

Repeat drying and calcination 
C0.061:

$$
\begin{aligned}
& 30 \text { wt\% Co } \\
& 0.5 \text { wt\% Ru } \\
& \gamma \text {-alumina }
\end{aligned}
$$

Ru1-promoted catalyst similar to Co.053 with $30 \%$ Co.

\section{Preparation Procedure:}

Calcine the $\gamma$-alumina at $500^{\circ} \mathrm{C}$ for $10 \mathrm{hrs}$. Use Vista B alumina. Presieve to $>38$ microns (400-0 mesh).

Impregnate the support in 2 steps with an aqueous solution of Co nitrate $\left[\mathrm{Co}\left(\mathrm{NO}_{3}\right)_{2} \cdot 6 \mathrm{H}_{2} \mathrm{O}\right]$, and $\mathrm{Ru}$ nitrosyl nitrate using an appropriate quantity to get incipient wemess (ca. $1.2 \mathrm{ml} / \mathrm{g}$ ) with $60 \%$ of the desired loading of $\mathrm{Co}$ and $\mathrm{Ru}$ in the first step.

Dry in air at $115^{\circ} \mathrm{C}$ for 12 hours and calcine in air at $300^{\circ} \mathrm{C}$ for 2 hours (heating rate of ca. $1^{\circ} \mathrm{C} / \min$ to $300^{\circ} \mathrm{C}$ ).

In the second step, impregnate the remaining $40 \%$ of the Co and Ru. Repeat the drying and calcination procedures.

Reduction Procedure before Reaction:

Reduce the catalyst in a pure hydrogen flow of $3000 \mathrm{cc} / \mathrm{g} / \mathrm{hr}$ by heating at $1^{\circ} \mathrm{C} / \mathrm{min}$ to $350^{\circ} \mathrm{C}$ and holding for $10 \mathrm{hrs}$. 


\begin{tabular}{|c|c|c|c|c|}
\hline Catris & Med & Oct-10-94 & Ainininit & $250.0 \mathrm{~g}$ \\
\hline ) & \% & man: & 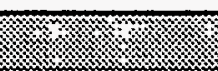 & (m) \\
\hline (\%wt) & 30 & 0.5 & & \\
\hline
\end{tabular}

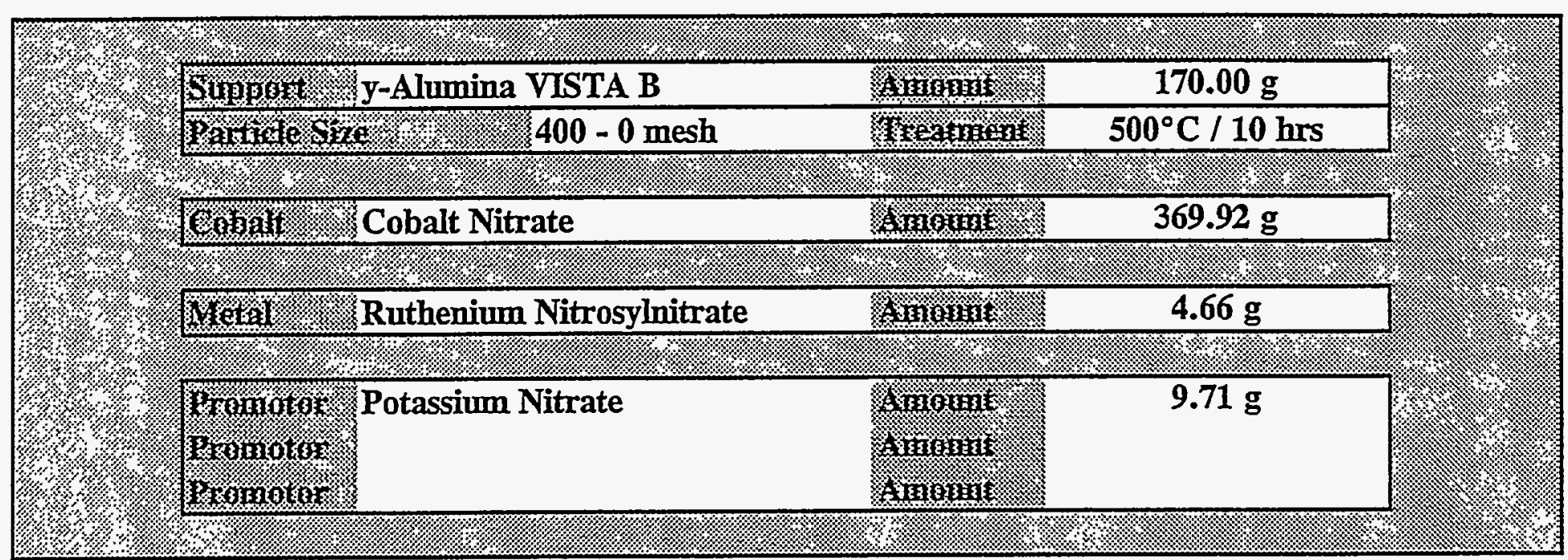

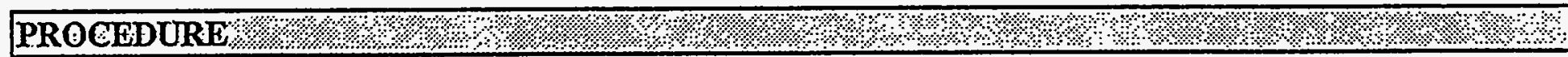
Preparation $\%: \%$ X Incipient Wetness

Ion Exchange Other

Notes Incipient wetness: aqueous solution - ca. $1.2 \mathrm{ml} / \mathrm{g}-60 \%$ of loading in first step Dry catalyst precursor in an oven at $115^{\circ} \mathrm{C} / 12 \mathrm{hrs}$ - calcine $300^{\circ} \mathrm{C} / 2 \mathrm{hrs}$ Incipient wetness: aqueous solution - ca. $1.2 \mathrm{ml} / \mathrm{g}$ - remaining $40 \%$ of loading Repeat drying and calcnation 
C0.062: $\quad 30 \mathrm{wt} \% \mathrm{Co}$

$0.5 \mathrm{wt} \% \mathrm{Ru}$

$1.5 \mathrm{wt} \% \mathrm{~K}$

$\cdot \gamma$-alumina

$\mathrm{Ru} / \mathrm{K}$-promoted catalyst similar to Co.029 with $1.5 \% \mathrm{~K}$

Preparation Procedure:

Calcine the $\gamma$-alumina at $500^{\circ} \mathrm{C}$ for $10 \mathrm{hrs}$. Use Vista $\mathrm{B}$ alumina. Presieve to $>38$ microns (400-0 mesh).

Impregnate the support in 2 steps with an aqueous solution of Co nitrate [Co $\left.\left(\mathrm{NO}_{3}\right)_{2} \cdot 6 \mathrm{H}_{2} \mathrm{O}\right]$, Ru nitrosyl nitrate, and $\mathrm{K}$ nitrate using an appropriate quantity to get incipient wemess (cr. $1.2 \mathrm{ml} / \mathrm{g}$ ) with $60 \%$ of the desired loading of $\mathrm{Co}, \mathrm{Ru}$, and $\mathrm{K}$ in the first step.

Dry in air at $115^{\circ} \mathrm{C}$ for 12 hours and calcine in air at $300^{\circ} \mathrm{C}$ for 2 hours (heating rate of ca $1^{\circ} \mathrm{C} / \min$ to $300^{\circ} \mathrm{C}$ ).

In the second step, impregnate the remaining $40 \%$ of the $\mathrm{Co}, \mathrm{R} u$, and $\mathrm{K}$ Repeat the drying and calcination procedures.

Reduction Procedure before Reaction:

Reduce the catalyst in a pure hydrogen flow of $3000 \mathrm{cc} / \mathrm{g} / \mathrm{hr}$ by heating at $1^{\circ} \mathrm{C} / \mathrm{min}$ to $350^{\circ} \mathrm{C}$ and holding for $10 \mathrm{hrs}$. 
Co.063: $\quad 20 \mathrm{wt} \%$ Co

$8.5 \mathrm{wt} \% \mathrm{La}$

$\mathrm{Al}_{2} \mathrm{O}_{3}$

La-promoted $\mathrm{Al}_{2} \mathrm{O}_{3}$-supported catalyst comparable to $\mathrm{Co} .056$ with $\mathrm{SiO}_{2}$ replaced by $\mathrm{Al}_{2} \mathrm{O}_{3}$ as the support. One-step impregnation of alumina with lanthanum nitrate followed by onestep impregnation with cobalt nitrate solution.

\section{Preparation Procedure:}

Calcine the $\gamma$-alumina at $500^{\circ} \mathrm{C}$ for $10 \mathrm{hrs}$. Use Vista B alumina. Presieve to $>38$ microns (400-0 mesh).

Impregnate the support with an aqueous solution of La nitrate using an appropriate quantity to get incipient wetness with the desired loading of $\mathrm{La}$.

Dry the La-loaded $\mathrm{Al}_{2} \mathrm{O}_{3}$ in an oven for $5 \mathrm{hrs}$ at $115^{\circ} \mathrm{C}$ with moderate stirring.

\# Calcine the dried support in air by raising its temperature at a heating rate of ca. $1^{\circ} \mathrm{C} / \mathrm{min}$ to $300^{\circ} \mathrm{C}$ and holding for $2 \mathrm{hrs}$.

Impregnate the La-loaded alumina with an aqueous solution of Co nitrate $\left[\mathrm{Co}\left(\mathrm{NO}_{3}\right)_{2} \cdot 6 \mathrm{H}_{2} \mathrm{O}\right]$ using an appropriate quantity to get incipient wetness with the desired loading of $\mathrm{Co}$.

\# Dry the catalyst precursor in an oven for $5 \mathrm{hrs}$ at $115^{\circ} \mathrm{C}$ with moderate stirring.

\# Calcine the dried catalyst in air by raising its temperature at a heating rate of ca. $1^{\circ} \mathrm{C} / \mathrm{min}$ to $300^{\circ} \mathrm{C}$ and holding for $2 \mathrm{hrs}$.

\section{Reduction Procedure before Reaction:}

Reduce the catalyst in a pure hydrogen flow of $3000 \mathrm{cc} / \mathrm{g} / \mathrm{hr}$ by heating at $1^{\circ} \mathrm{C} / \mathrm{min}$ to $350^{\circ} \mathrm{C}$ and holding for $10 \mathrm{hrs}$. 


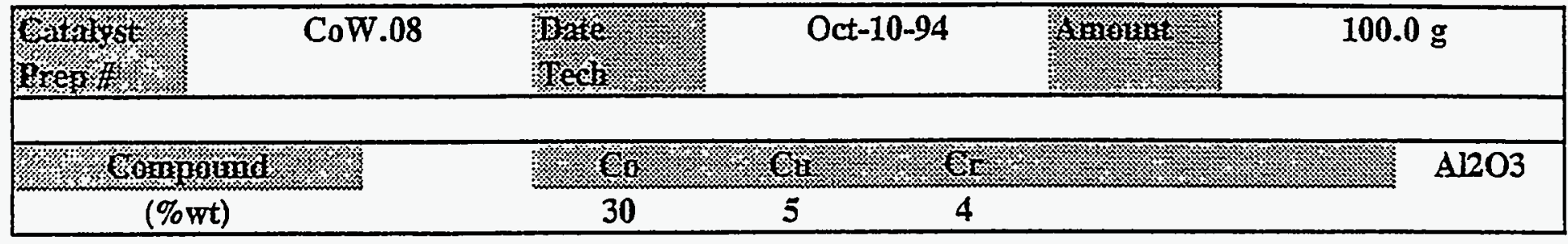

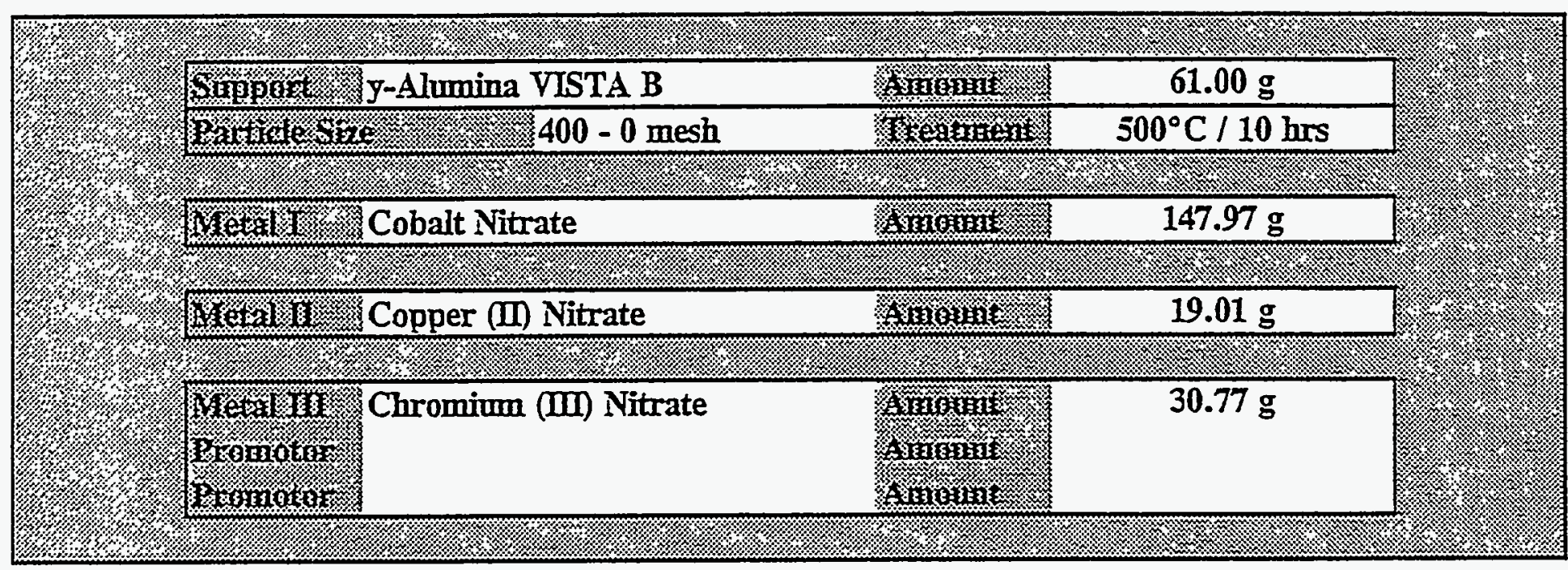

\begin{tabular}{|c|c|c|}
\hline 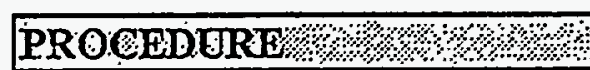 & 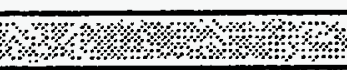 & \%ः \\
\hline Preparation $\%$ \% $\quad \mathrm{X}$ & $\begin{array}{l}\text { Incipient Wetness } \\
\text { Ion Exchange }\end{array}$ & $\begin{array}{l}\text { Wet Impregnation } \\
\text { Other. }\end{array}$ \\
\hline
\end{tabular}

Nötes 2 Incipient wetness: aqueous solution $\mathrm{Cu}+\mathrm{Cr}$, ca. $1.2 \mathrm{ml} / \mathrm{g}$

Dry catalyst precursor in an oven $110^{\circ} \mathrm{C} / 16 \mathrm{hrs} /$ Calcine $750^{\circ} \mathrm{C} 24 \mathrm{hrs}$.

Incipient wetness: aqueous solution $\mathrm{Co}$.

Dry catalyst precursor in an oven $115^{\circ} \mathrm{C} / 5 \mathrm{hrs}$.

Ealcination : $300^{\circ} \mathrm{C}$ Time $10 \mathrm{hrs}$


CoW.08

$$
\begin{aligned}
& 30 \text { wt\% Co } \\
& 5 \text { wt\% Cu } \\
& 4 \text { wt\% Cr } \\
& y \text {-alumina }
\end{aligned}
$$

Cobalt impregnation on calcined $\mathrm{Cu}-\mathrm{Cr} / \gamma$-alumina. Similar to CoW.05, but with $30 \%$ Co.

\section{Preparation Procedure}

Calcine $\gamma$-alumina at $500^{\circ} \mathrm{C}$ for 10 hrs. Use Vista B alumina. Presieve to $>38$ microns (400-0 mesh).

Impregnate the support with an aqueous solution of $\mathrm{Cu}\left(\mathrm{NO}_{3}\right)_{2} \mathrm{xH}_{2} \mathrm{O}$, and $\mathrm{Cr}\left(\mathrm{NO}_{3}\right)_{3} .9 \mathrm{H}_{2} \mathrm{O}$ using appropriate quantity to get incipient wetness (ca. $1.2 \mathrm{ml} / \mathrm{g}$ ) with the desired loading of $\mathrm{Cu}$ and $\mathrm{Cr}$.

Dry the catalyst precursor in an oven for 16 hours at $110^{\circ} \mathrm{C}$.

The dried catalyst is then calcined in air by raising its temperature at a heating rate of c. $1^{\circ} \mathrm{C} / \mathrm{min}$ to $750^{\circ} \mathrm{C}$ and holding for 24 hours.

Impregnate the $\mathrm{Cu}-\mathrm{Cr} / \mathrm{Al}_{2} \mathrm{O}_{3}$ with an aqueous solution of $\mathrm{Co}$ nitrate $\left[\mathrm{Co}\left(\mathrm{NO}_{3}\right)_{2} \cdot \mathrm{H}_{2} \mathrm{O}\right]$ using an appropriate quantity to get incipient wetness with the desired loading of $\mathrm{Co}$.

Dry the catalyst precursor in an oven for 5 hrs at $115^{\circ} \mathrm{C}$ with moderate stirring.

Calcine the dried catalyst in air by raising its temperature at a heating rate of $\mathrm{ca}$. $1^{\circ} \mathrm{C} / \min$ to $300^{\circ} \mathrm{C}$ and holding for $10 \mathrm{hrs}$.

\section{Reduction Procedure before Reaction:}

Heat the catalyst in inert gas to $120^{\circ} \mathrm{C}$ at a rate of $1^{\circ} \mathrm{C} /$ min then start adding hydrogen to give a concentration of $0.5 \%$ at the bed inlet. Raise the catalyst bed temperature to $165^{\circ} \mathrm{C}$ at a rate of $\mathrm{ca} .30^{\circ} \mathrm{C} / \mathrm{hr}$. When the temperature of the bed has reached $160^{\circ} \mathrm{C}$ increase the hydrogen concentration in the carrier gas to $1.0 \%$. As the reduction proceeds and the temperature rise begins to diminish, the inlet temperature may be raised to $200^{\circ} \mathrm{C}$. The inlet hydrogen concentration can then be increased to about $3-5 \%$, provided that the maximum temperature limit of $230^{\circ} \mathrm{C}$ is not exceeded. When the reduction appears to be complete the inlet temperature should be raised to $230^{\circ} \mathrm{C}$ and the inlet hydrogen concentration raised to ca. $20 \%$. 


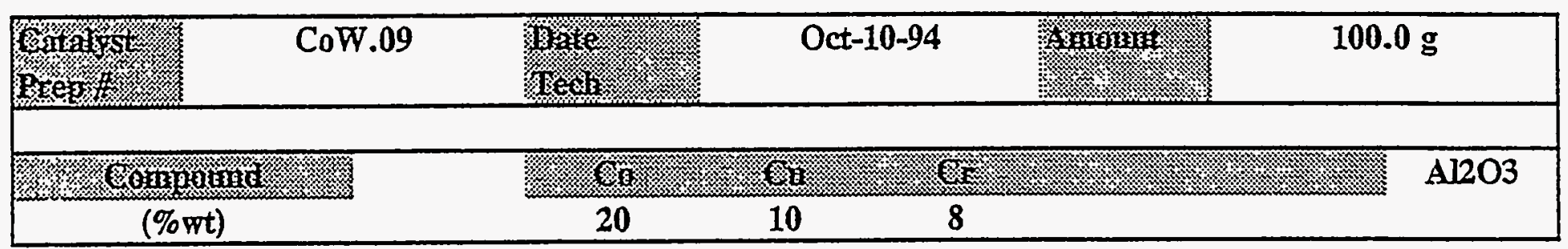

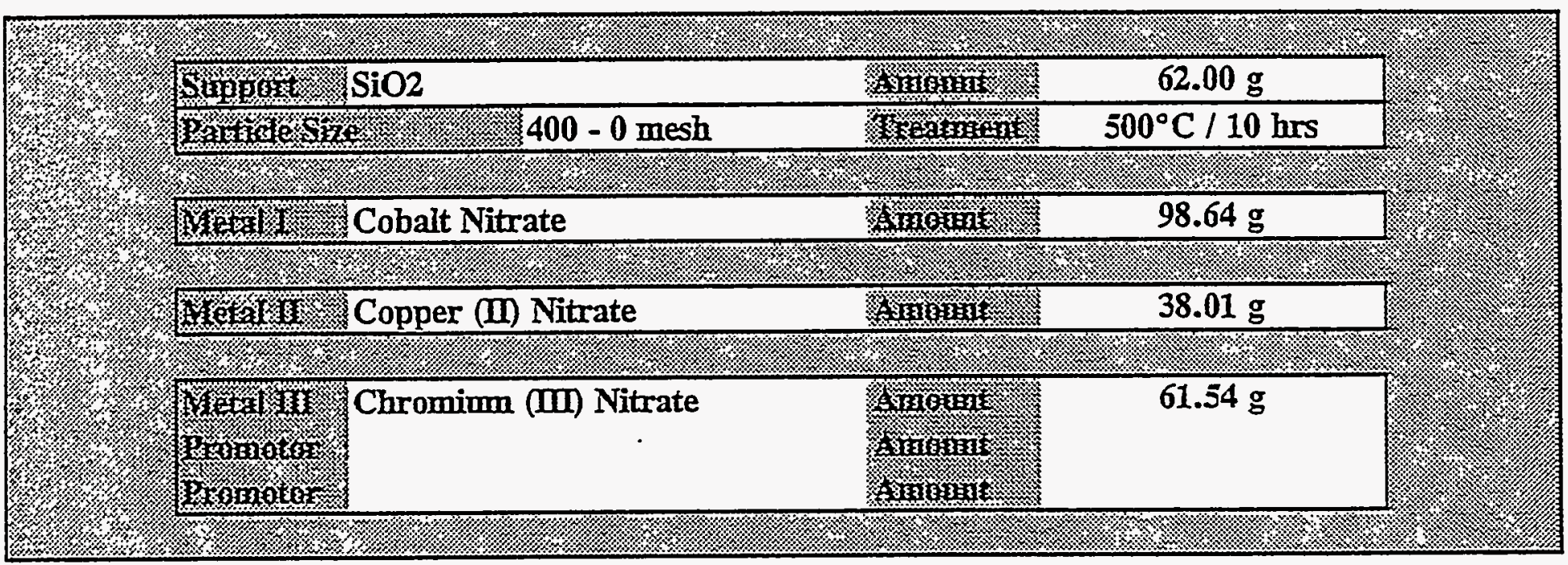

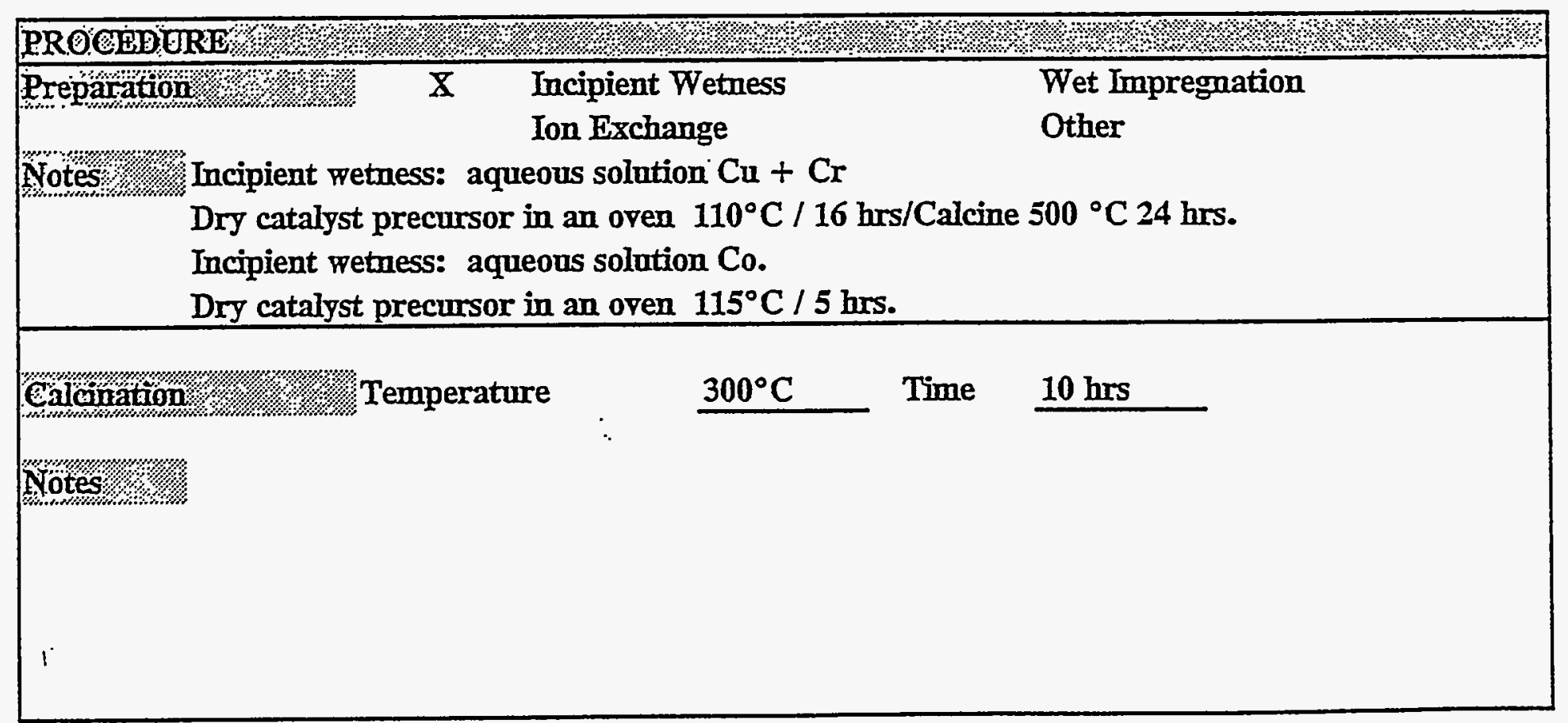


CoW.09
20 wt\% Co
$10 \mathrm{wt} \% \mathrm{Cu}$
8 wt\% $\mathrm{Cr}$
Silica

Cobalt impregnation on calcined $\mathrm{Cu}-\mathrm{Cr} /$ Silica. Similar to $\mathrm{CoW} .06$, but with $10 \% \mathrm{Cu}$ and $8 \%$ Cr.

\section{Preparation Procedure}

Calcine silica at $500^{\circ} \mathrm{C}$ for $10 \mathrm{hrs}$. Use Davisson Grade 952 silica. Presieve to $>38$ microns (400-0 mesh).

Impregnate the support with an aqueous solution of $\mathrm{Cu}\left(\mathrm{NO}_{3}\right)_{2} \mathrm{xH}_{2} \mathrm{O}$, and $\mathrm{Cr}\left(\mathrm{NO}_{3}\right)_{3} \cdot 9 \mathrm{H}_{2} \mathrm{O}$ using appropriate quantity to get incipient wetness (ca. $1.2 \mathrm{ml} / \mathrm{g}$ ) with the desired loading of $\mathrm{Cu}$ and $\mathrm{Cr}$.

Dry the catalyst precursor in an oven for 16 hours at $110^{\circ} \mathrm{C}$.

The dried catalyst precursor is then calcined in air by raising its temperature at a heating rate of $\mathrm{ca} .1^{\circ} \mathrm{C} / \mathrm{min}$ to $500^{\circ} \mathrm{C}$ and holding for 24 hours.

Impregnate the $\mathrm{Cu}-\mathrm{Cr} / \mathrm{SiO}_{2}$ with an aqueous solution of $\mathrm{Co}$ nitrate $\left[\mathrm{Co}\left(\mathrm{NO}_{3}\right)_{2} \cdot 6 \mathrm{H}_{2} \mathrm{O}\right.$ ] using an appropriate quantity to get incipient wemess with the desired loading of $\mathrm{Co}$.

Dry the catalyst precursor in an oven for 5 hrs at $115^{\circ} \mathrm{C}$ with moderate sirring.

Calcine the dried catalyst in air by raising its temperature at a heating rate of $\mathrm{cr}$ $1^{\circ} \mathrm{C} / \min$ to $300^{\circ} \mathrm{C}$ and holding for 10 hrs.

\section{Reduction Procedure before Reaction:}

Heat the catalyst in inert gas to $120^{\circ} \mathrm{C}$ at a rate of $1^{\circ} \mathrm{C} / \mathrm{min}$ then start adding hydrogen to give a concentration of $0.5 \%$ at the bed inlet. Raise the catalyst bed temperature to $165^{\circ} \mathrm{C}$ at a rate of $\mathrm{ca} 30^{\circ} \mathrm{C} / \mathrm{hr}$. When the temperature of the bed has reached $160^{\circ} \mathrm{C}$ increase the hydrogen concentration in the carrier gas to $1.0 \%$. As the reduction proceeds and the temperature rise begins to diminish, the inlet temperature may be raised to $200^{\circ} \mathrm{C}$. The inlet hydrogen concentration can then be increased to about $3-5 \%$, provided that the maximum temperature limit of $230^{\circ} \mathrm{C}$ is not exceeded. When the reduction appears to be complete the inlet temperature should be raised to $230^{\circ} \mathrm{C}$ and the inlet hydrogen concentration raised to ca. $20 \%$. 


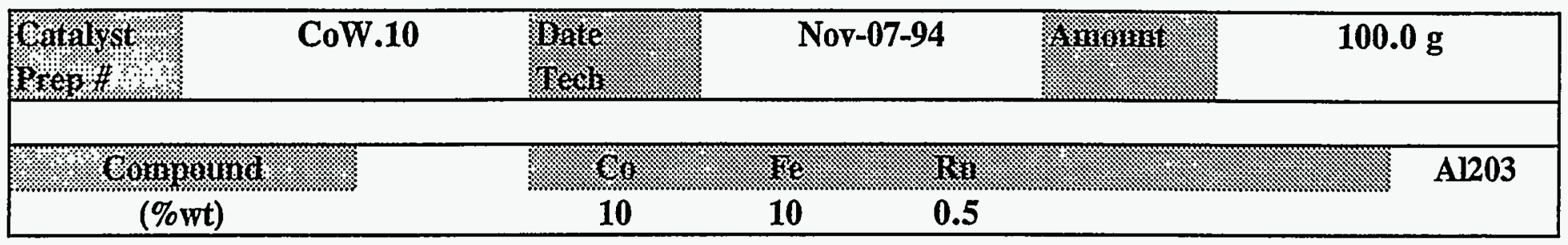

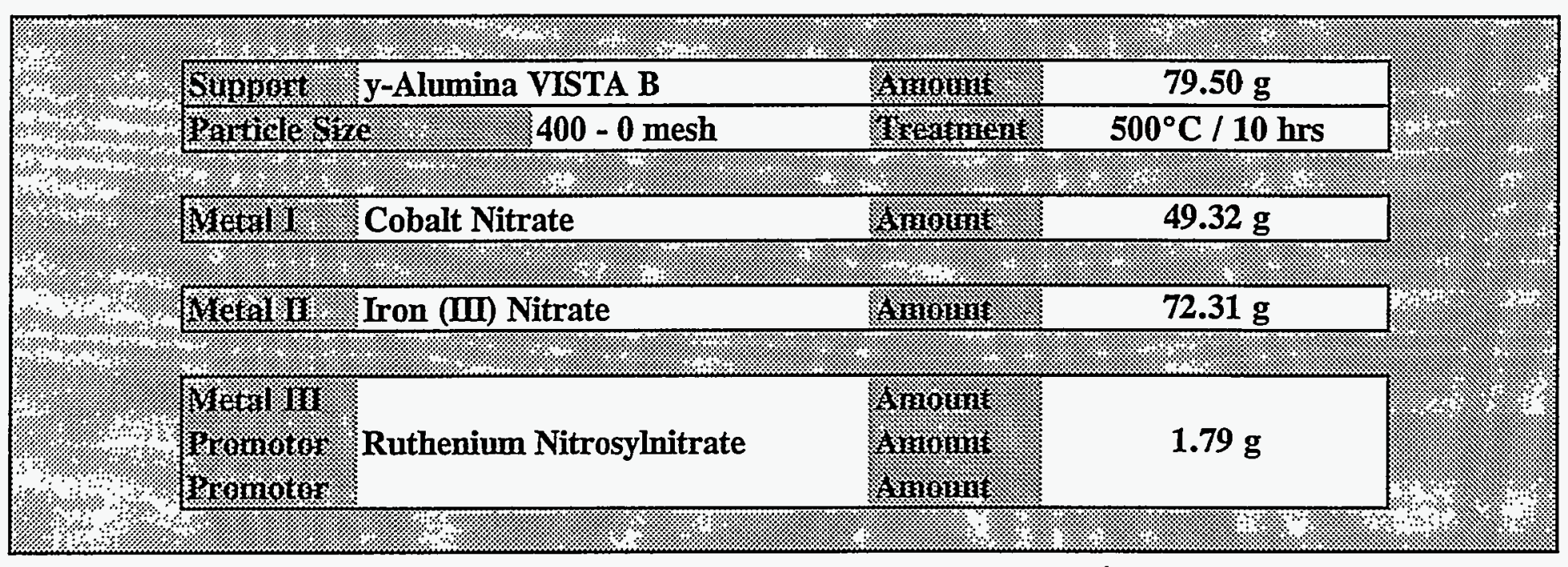

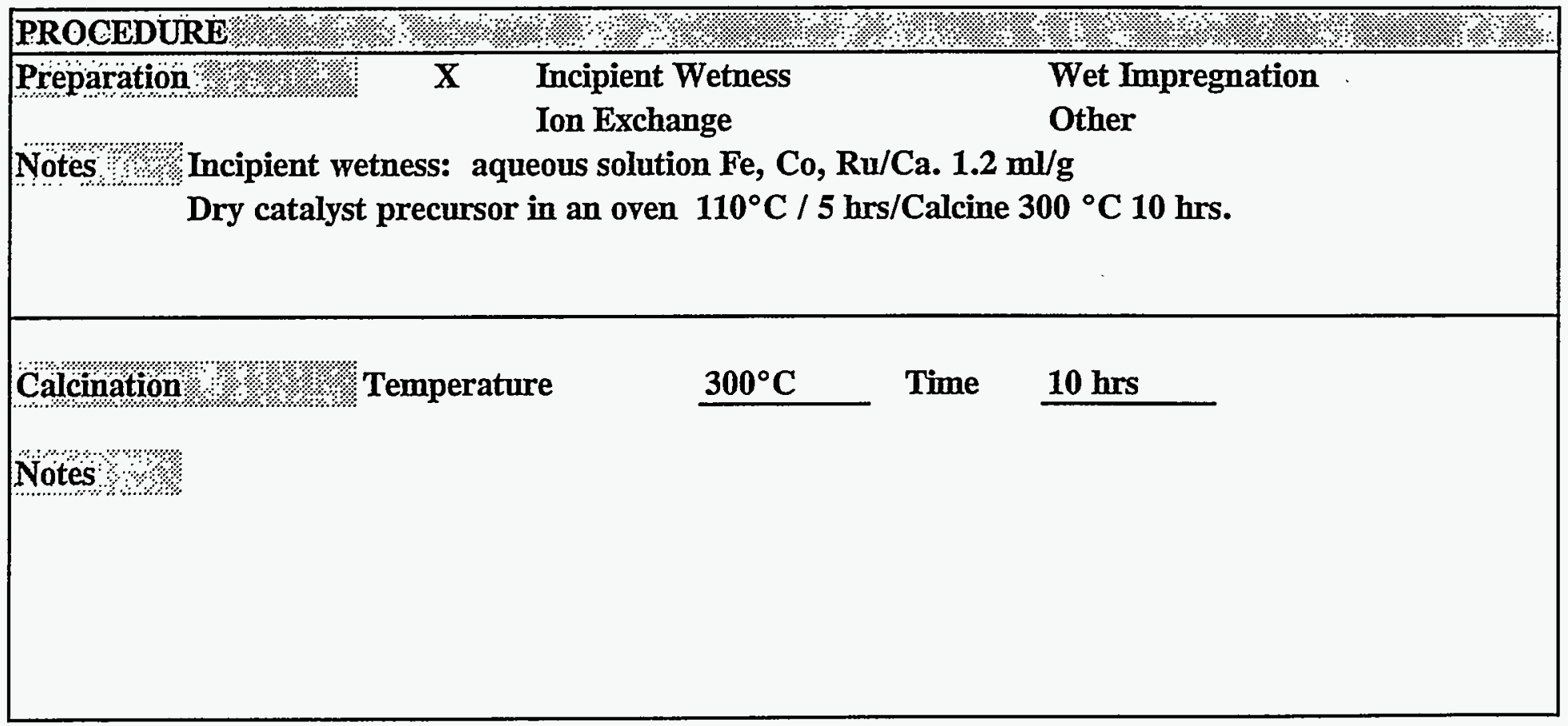


CoW.10 $\quad 10 \mathrm{wt} \% \mathrm{Fe}$

$10 \mathrm{wt} \% \mathrm{Co}$

$0.5 \mathrm{wt} \% \mathrm{Ru}$

$\gamma$-alumina

Ru promoted cobalt/iron impregnated on calcined $\gamma$-alumina

\section{Preparation Procedure}

Calcine $\gamma$-alumina at $500^{\circ} \mathrm{C}$ for $10 \mathrm{hrs}$. Use Vista $\mathrm{B}$ alumina. Presieve to $>38$ microns (400-0 mesh).

Impregnate the support with an aqueous solution of $\mathrm{Fe}$ nitrate $\left[\mathrm{Fe}\left(\mathrm{NO}_{3}\right)_{3} \cdot 9 \mathrm{H}_{2} \mathrm{O}\right]$, Co nitrate $\left[\mathrm{Co}\left(\mathrm{NO}_{3}\right)_{2} \cdot 6 \mathrm{H}_{2} \mathrm{O}\right]$ and $\mathrm{Ru}$ nitrosylnitrate $\left[\mathrm{Ru}(\mathrm{NO})\left(\mathrm{NO}_{3}\right)_{3} \cdot 2 \mathrm{H}_{2} \mathrm{O}\right]$ using appropriate quantities to get incipient wetness (ca. $1.2 \mathrm{ml} / \mathrm{g}$ ) with the desired loading of $\mathrm{Fe}, \mathrm{Co}$, and $\mathrm{Ru}$.

Dry the catalyst precursor in an oven for $5 \mathrm{hrs}$ at $115^{\circ} \mathrm{C}$ with moderate stirring.

Calcine the dried catalyst in air by raising its temperature at a heating rate of ca. $1^{\circ} \mathrm{C} / \min$ to $300^{\circ} \mathrm{C}$ and holding for $10 \mathrm{hrs}$.

\section{Reduction Procedure before Reaction:}

Reduce the catalyst in pure hydrogen flow of $3000 \mathrm{cc} / \mathrm{g} / \mathrm{hr}$ by heating at $1^{\circ} \mathrm{C} / \mathrm{min}$ to $350^{\circ} \mathrm{C}$ and holding for $10 \mathrm{hrs}$. 


\begin{tabular}{|c|c|c|c|c|c|}
\hline Catrost: & CoW.11 & $\frac{189}{16-4}$ & Nov-07-94 & 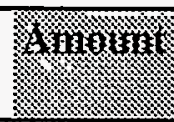 & $100.0 \mathrm{~g}$ \\
\hline 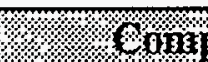 & $\sqrt{3}$ & 80 & 18 & In & Al2O3 \\
\hline$(\%$ & & 10 & 10 & 0.5 & \\
\hline
\end{tabular}

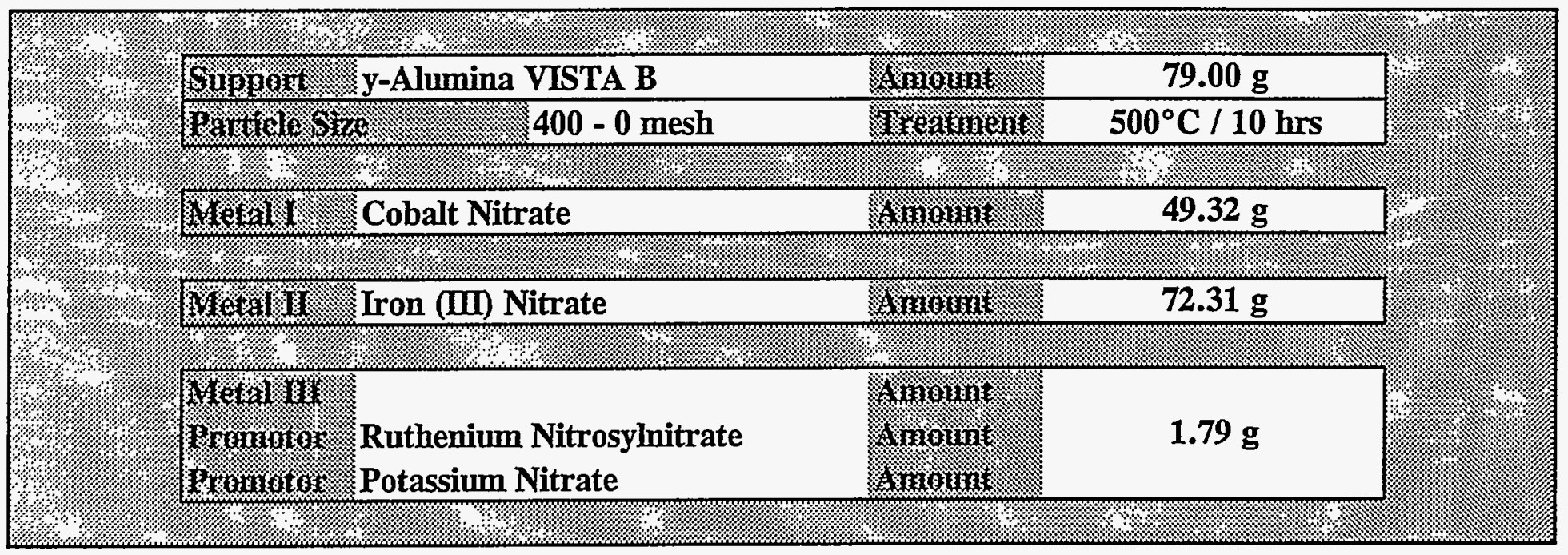

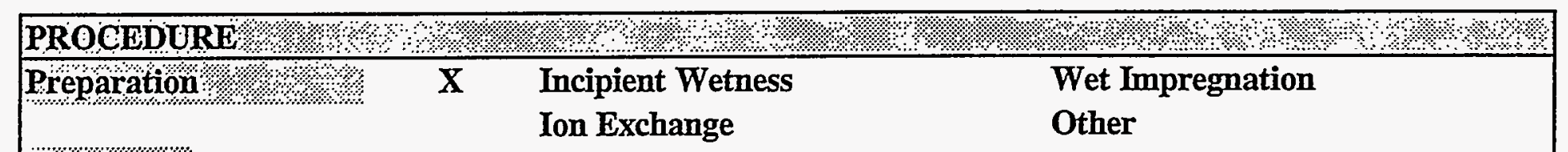

Notes / Incipient wetness: aqueous solution $\mathrm{Fe}, \mathrm{Co}, \mathrm{Ru}, \mathrm{K} / \mathrm{Ca} .1 .2 \mathrm{~g} / \mathrm{ml}$

Dry catalyst precursor in an oven $115^{\circ} \mathrm{C} / 5 \mathrm{hrs} /$ Calcine $300^{\circ} \mathrm{C} 10 \mathrm{hrs}$.

Calcination $1 \%$ Temperature $\quad 300^{\circ} \mathrm{C}$ Time $10 \mathrm{hrs}$


CoW.11 $10 \mathrm{wt} \% \mathrm{Fe}$

$10 \mathrm{wt} \% \mathrm{Co}$

$0.5 \mathrm{wt} \% \mathrm{Ru}$

0.5 wt\% K

$\gamma$-alumina

$\mathrm{Ru}$ and $\mathrm{K}$ promoted cobalt/iron impregnated on calcined $\gamma$-alumina

Preparation Procedure

Calcine $\gamma$-alumina at $500^{\circ} \mathrm{C}$ for $10 \mathrm{hrs}$. Use Vista $\mathrm{B}$ alumina. Presieve to $>38$ microns (400-0 mesh).

Impregnate the support with an aqueous solution of Fe nitrate $\left[\mathrm{Fe}\left(\mathrm{NO}_{3}\right)_{3} \cdot 9 \mathrm{H}_{2} \mathrm{O}\right]$, Co nitrate $\left[\mathrm{Co}\left(\mathrm{NO}_{3}\right)_{2} \cdot 6 \mathrm{H}_{2} \mathrm{O}\right]$, $\mathrm{Ru}$ nitrosylnitrate $\left[\mathrm{Ru}(\mathrm{NO})\left(\mathrm{NO}_{3}\right)_{3} \cdot 2 \mathrm{H}_{2} \mathrm{O}\right]$, and $\mathrm{K}$ nitrate $\left[\mathrm{KNO}_{3}\right]$ using appropriate quantities to get incipient wetness (ca. $1.2 \mathrm{ml} / \mathrm{g}$ ) with the desired loading of $\mathrm{Fe}, \mathrm{Co}, \mathrm{Ru}$, and $\mathrm{K}$.

Dry the catalyst precursor in an oven for 5 hrs at $115^{\circ} \mathrm{C}$ with moderate stirring.

Calcine the dried catalyst in air by raising its temperature at a heating rate of ca. $1^{\circ} \mathrm{C} / \min$ to $300^{\circ} \mathrm{C}$ and holding for $10 \mathrm{hrs}$.

Reduction Procedure before Reaction:

Reduce the catalyst in pure hydrogen flow of $3000 \mathrm{cc} / \mathrm{g} / \mathrm{hr}$ by heating at $1^{\circ} \mathrm{C} / \mathrm{min}$ to $350^{\circ} \mathrm{C}$ and holding for $10 \mathrm{hrs}$. 


\begin{tabular}{|c|c|c|c|c|}
\hline Cataly & Non: & Nov-07-94 & 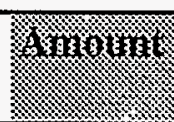 & $100.0 \mathrm{~g}$ \\
\hline 71 Compound & 8 & 72 & & $\overline{\mathrm{SiO} 2}$ \\
\hline (\%wt) & 10 & 10 & & \\
\hline
\end{tabular}

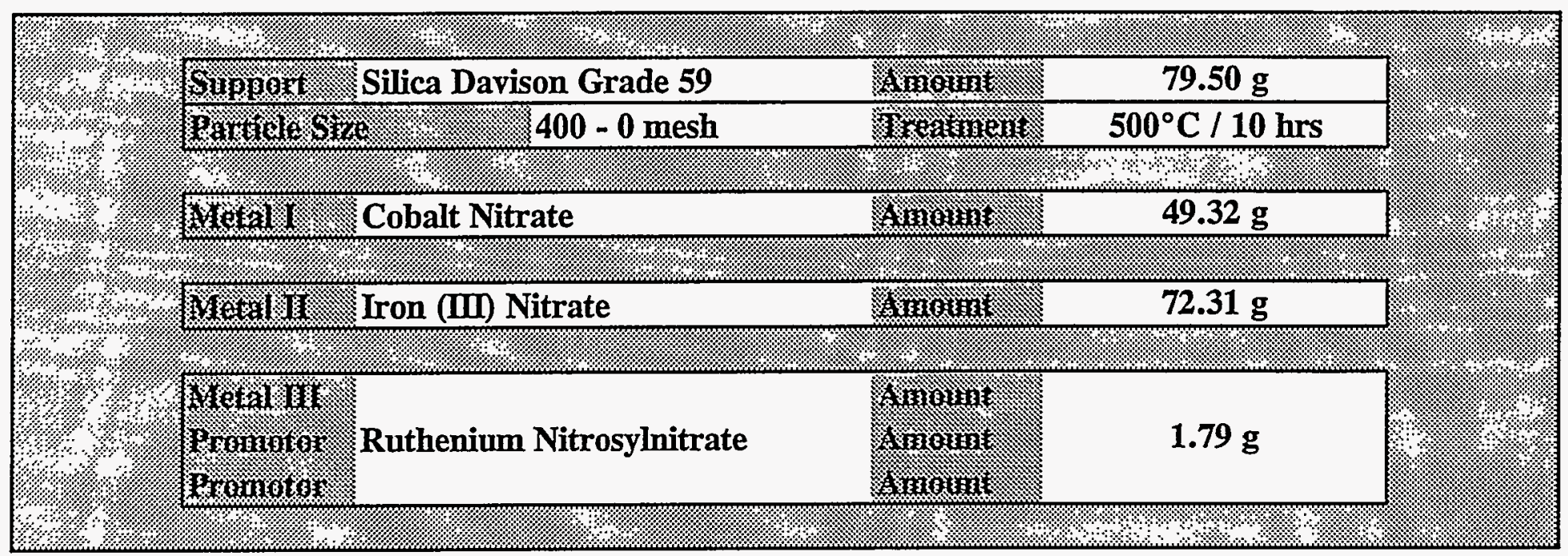

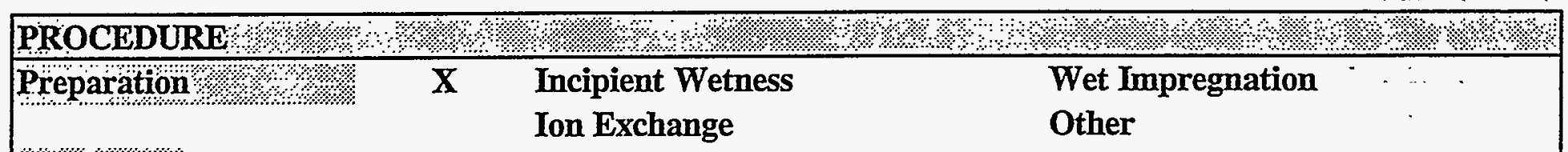
Notes $\%$ Incipient wetness: aqueous solution $\mathrm{Co}, \mathrm{Fe}, \mathrm{Ru}$
Dry catalyst precursor in an oven $115^{\circ} \mathrm{C} / 5 \mathrm{hrs} /$ Calcine $300{ }^{\circ} \mathrm{C} 10 \mathrm{hrs}$.

Calcination 1 . 1 Temperature $\quad \underline{300^{\circ} \mathrm{C}}$ Time $10 \mathrm{hrs}$

\section{Notes}


CoW.12 $10 \mathrm{wt} \% \mathrm{Fe}$

$10 \mathrm{wt} \% \mathrm{Co}$

0.5 wt\% Ru

Silica

$\mathrm{Ru}$ promoted cobalt/iron impregnated on calcined silica

Preparation Procedure

Calcine silica at $500^{\circ} \mathrm{C}$ for $10 \mathrm{hrs}$. Use Davisson Grade 952 silica. Presieve to $>38$ microns (400-0 mesh).

Impregnate the support with an aqueous solution of $\mathrm{Fe}$ nitrate $\left[\mathrm{Fe}\left(\mathrm{NO}_{3}\right)_{3} \cdot 9 \mathrm{H}_{2} \mathrm{O}\right]$, $\mathrm{Co}$ nitrate $\left[\mathrm{Co}\left(\mathrm{NO}_{3}\right)_{2} \cdot 6 \mathrm{H}_{2} \mathrm{O}\right]$ and $\mathrm{Ru}$ nitrosylnitrate $\left[\mathrm{Ru}(\mathrm{NO})\left(\mathrm{NO}_{3}\right)_{3} \cdot 2 \mathrm{H}_{2} \mathrm{O}\right]$ using appropriate quantities to get incipient wetmess with the desired loading of $\mathrm{Fe}, \mathrm{Co}$, and Ru.

Dry the catalyst precursor in an oven for $5 \mathrm{hrs}$ at $115^{\circ} \mathrm{C}$ with moderate stirring.

Calcine the dried catalyst in air by raising its temperature at a heating rate of ca. $1^{\circ} \mathrm{C} / \mathrm{min}$ to $300^{\circ} \mathrm{C}$ and holding for $10 \mathrm{hrs}$.

Reduction Procedure before Reaction:

Reduce the catalyst in pure hydrogen flow of $3000 \mathrm{cc} / \mathrm{g} / \mathrm{hr}$ by heating at $1^{\circ} \mathrm{C} / \mathrm{min}$ to $250^{\circ} \mathrm{C}$ and holding for $10 \mathrm{hrs}$. 
APPENDIX B

Fixed Bed Reaction Data 


\section{Co.004 - Run \#6}

\begin{tabular}{|c|c|c|c|c|}
\hline Co wt\% & NM & wt \% & Promotor wt\% & Support \\
\hline 20 & $\mathbf{R u}$ & 0.43 & $\mathrm{La2O3} 1.00$ & $\mathrm{Al2O3}$ \\
\hline
\end{tabular}

\section{SUMMARY REACTION DATA}

Reaction Conditions:

$$
\begin{array}{ll}
\mathrm{P}=1.0 \mathrm{~atm} & \mathrm{CO} 2(\mathrm{~g} / \mathrm{g} \mathrm{cat} / \mathrm{hr})=0.044 \\
\mathrm{~T}=220^{\circ} \mathrm{C} & \mathrm{CO} 2(\% \text { of } \mathrm{CO})=0.2 \\
\mathrm{H} 2 / \mathrm{CO}=2 & \mathrm{O} / \mathrm{P}=1.60 \\
\text { weight of catalyst }=0.167 \mathrm{~g} & \\
\text { WHSV }=15.371 / \mathrm{hr} & \\
\text { time on stream }=27.5 \mathrm{hrs} &
\end{array}
$$

\begin{tabular}{|l|c|}
\hline \hline $\mathrm{CO}$ conversion (\%) & 6.9 \\
\hline rate (g CH2/g cat/hr) & 0.47 \\
\hline alpha & 0.61 \\
\hline $\mathrm{C} 1$ (wt\%) & 27.8 \\
\hline $\mathrm{C} 2-\mathrm{C} 4(\mathrm{wt} \%)$ & 29.9 \\
\hline $\mathrm{C} 5-\mathrm{C} 12(\mathrm{wt} \%)$ & 40.6 \\
\hline $\mathrm{C} 13+(\mathrm{wt} \%)$ & 1.8 \\
\hline
\end{tabular}

* Calcined in Nitrogen 


\section{Performance of Co.004 \\ Dates: 10/10/94 - 10/11/94 Run \#6}

flow rate $=90.0 \mathrm{cc} / \mathrm{min}$, loading $=0.2 \mathrm{~g}, \mathrm{WHSV}=15.41 / \mathrm{hr}, \mathrm{H} 2 / \mathrm{CO}$ ratio in feed $=2$

\begin{tabular}{|c|c|c|c|c|}
\hline time on stream, hr & 6.5 & 12.5 & 15.5 & 27.5 \\
\hline reaction temperature, ${ }^{\circ} \mathrm{C}$ & 220 & 220 & 220 & 220 \\
\hline pressure, atm & 1.0 & 1.0 & 1.0 & 1.0 \\
\hline flow, $\mathrm{cc} / \mathrm{min}$ & 90.0 & 90.0 & 90.0 & 90.0 \\
\hline \multicolumn{5}{|c|}{ C1 - C15 product distribution, weight \% } \\
\hline $\mathrm{C} 1$ & 25.97 & 28.71 & 26.79 & 27.79 \\
\hline $\mathrm{C} 2$ & 4.64 & 5.12 & 4.74 & 4.88 \\
\hline C3 & 12.26 & 13.28 & 12.38 & 12.61 \\
\hline $\mathrm{C} 4$ & 12.38 & 12.86 & 12.38 & 12.40 \\
\hline C5 & 11.59 & 10.91 & 11.50 & 11.28 \\
\hline C6 & 8.68 & 6.10 & 7.91 & 7.77 \\
\hline $\mathrm{C7}$ & 8.29 & 7.87 & 8.18 & 7.97 \\
\hline $\mathrm{C} 8$ & 5.58 & 5.37 & 5.63 & 5.38 \\
\hline C9 & 3.75 & 3.57 & 3.67 & 3.54 \\
\hline $\mathrm{C} 10$ & 2.46 & 2.42 & 2.53 & 2.41 \\
\hline $\mathrm{C} 11$ & 1.67 & 1.65 & 1.74 & 1.58 \\
\hline $\mathrm{C} 12$ & 1.05 & 1.02 & 1.03 & 0.96 \\
\hline $\mathrm{C} 13$ & 0.70 & 0.62 & 0.68 & 0.67 \\
\hline C14 & 0.57 & 0.35 & 0.54 & 0.51 \\
\hline C15 & 0.40 & 0.14 & 0.30 & 0.27 \\
\hline chain growth probability & 0.64 & 0.58 & 0.62 & 0.61 \\
\hline
\end{tabular}

C1 - C50 estimated total product distribution, weight \%

$\begin{array}{lcccc}\text { C1 } & 25.6 & 29.1 & 26.7 & 27.8 \\ \text { C2 - C4 } & 28.9 & 31.7 & 29.4 & 29.9 \\ \text { C5 - C12 } & 42.8 & 38.2 & 41.8 & 40.6 \\ \text { C13 - C50 } & 2.6 & 0.9 & 2.0 & 1.8\end{array}$

CO conversion, \%

rate, $\mathrm{g} \mathrm{CH} 2 / \mathrm{g} \mathrm{cat} / \mathrm{hr}$

$\mathrm{CO} 2$ formation, $\%$

$\begin{array}{llll}8.4 & 6.1 & 7.2 & 6.9 \\ 0.56 & 0.41 & 0.49 & 0.47 \\ 0.2 & 0.3 & 0.2 & 0.2\end{array}$


Time-on-Stream Plot for Co.004 - Run \#6

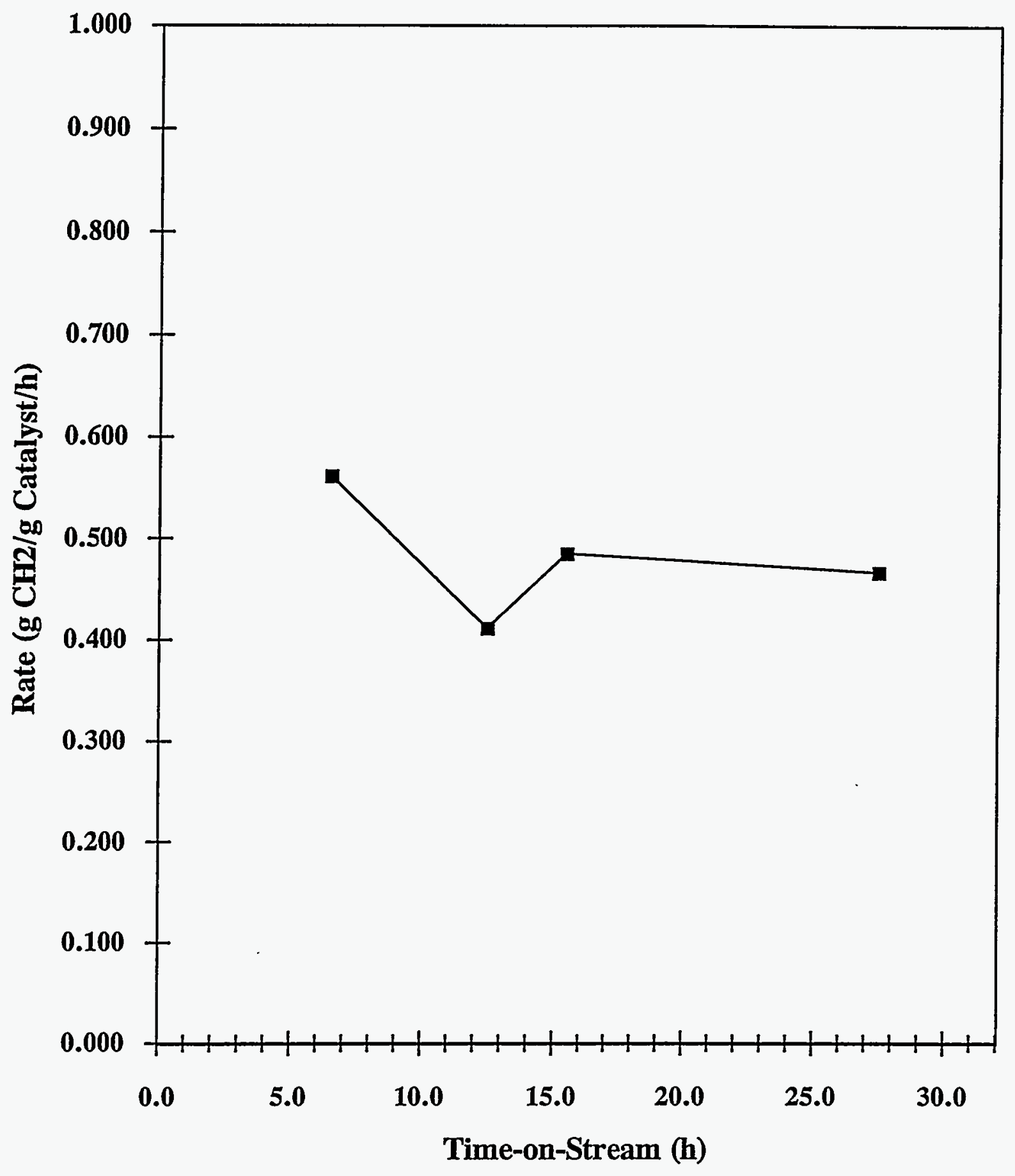


Schulz-Flory Plot for Co.004 - Run \#6

Time on Stream (hrs)

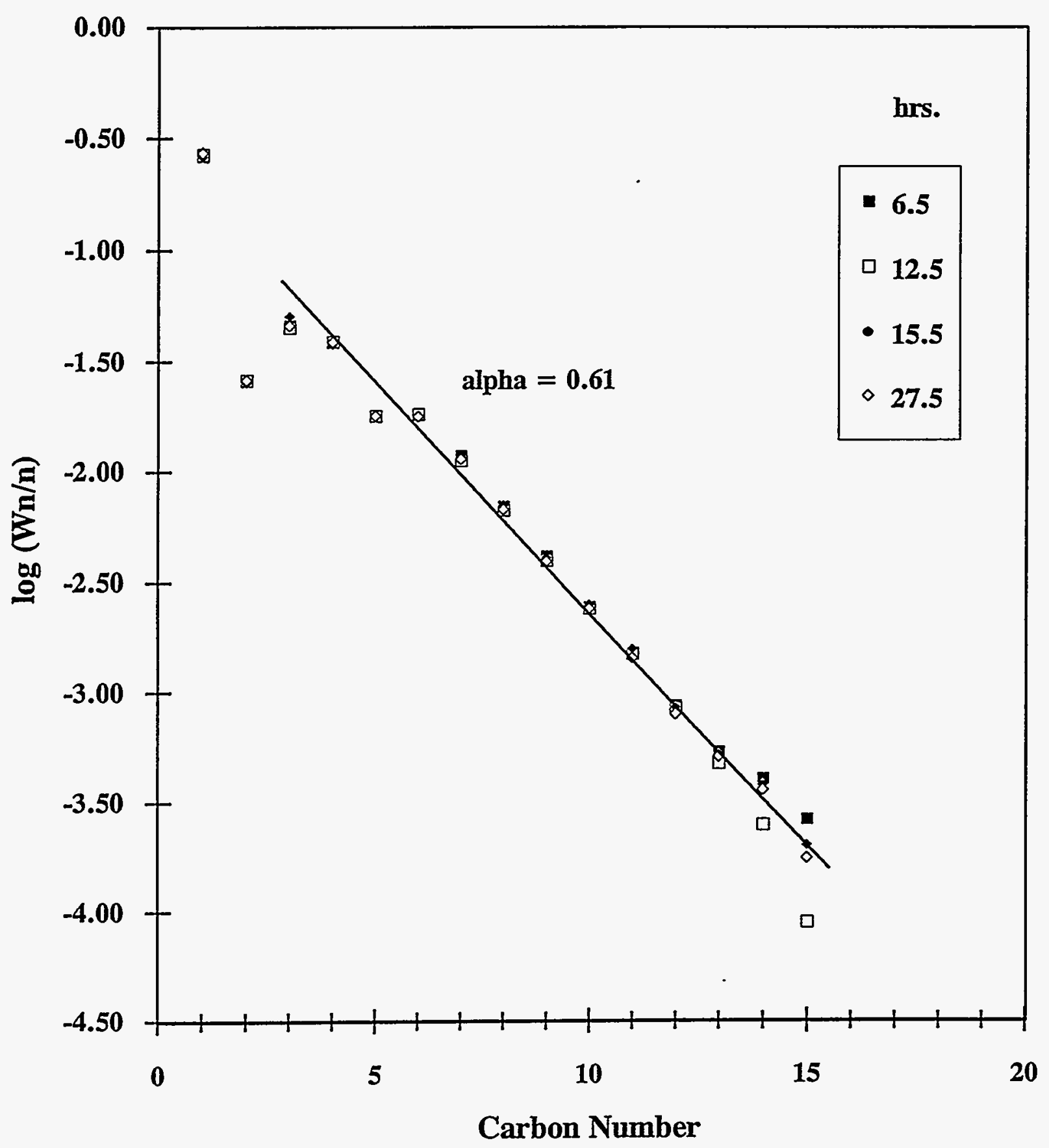




\section{Co.004 - Run \#7}

\begin{tabular}{|c|c|c|c|c|}
\hline Co wt\% & NM & wt \% & Promotor wt\% & Support \\
\hline 20 & $\mathbf{R u}$ & 0.43 & $\mathrm{La2O3} 1.00$ & $\mathrm{Al} 2 \mathrm{O3}$ \\
\hline
\end{tabular}

\section{SUMMARY REACTION DATA*}

Reaction Conditions:

$$
\begin{array}{ll}
\mathrm{P}=1.0 \mathrm{~atm} & \mathrm{CO} 2(\mathrm{~g} / \mathrm{g} \mathrm{cat} / \mathrm{hr})=0.042 \\
\mathrm{~T}=220^{\circ} \mathrm{C} & \mathrm{CO} 2(\% \text { of } \mathrm{CO})=0.2 \\
\mathrm{H} 2 / \mathrm{CO}=2 & \mathrm{O} / \mathrm{P}=3.01 \\
\text { weight of catalyst }=0.194 \mathrm{~g} & \\
\text { WHSV }=13.291 / \mathrm{hr} & \\
\text { time on stream }=27.5 \mathrm{hrs} &
\end{array}
$$

\begin{tabular}{|l|c|}
\hline CO conversion (\%) & 3.9 \\
\hline rate (g CH2/g cat/hr) & 0.23 \\
\hline alpha & 0.61 \\
\hline $\mathrm{C} 1$ (wt\%) & 28.8 \\
\hline $\mathrm{C} 2-\mathrm{C} 4$ (wt\%) & 29.3 \\
\hline $\mathrm{C} 5-\mathrm{C} 12(\mathrm{wt} \%)$ & 40.4 \\
\hline $\mathrm{C} 13+(w \mathrm{t} \%)$ & 1.5 \\
\hline
\end{tabular}

* Catalyst is reduced, calcined and rereduced 


\section{Performance of Co.004 \\ Dates: 10/24/94 - 10/25/94 Run \#7}

flow rate $=90.0 \mathrm{cc} / \mathrm{min}$, loading $=0.2 \mathrm{~g}, \mathrm{WHSV}=13.31 / \mathrm{hr}, \mathrm{H} 2 / \mathrm{CO}$ ratio in feed $=2$

\begin{tabular}{|c|c|c|c|c|c|c|}
\hline time on stream, hr & 0.5 & 3.5 & 9.5 & 21.5 & 24.5 & 27.5 \\
\hline reaction temperature, ${ }^{\circ} \mathrm{C}$ & 220 & 220 & 220 & 220 & 220 & 220 \\
\hline pressure, atm & 1.0 & 1.0 & 1.0 & 1.0 & 1.0 & 1.0 \\
\hline flow, cc/min & 90.0 & 90.0 & 90.0 & 90.0 & 90.0 & 90.0 \\
\hline \multicolumn{7}{|c|}{ C1 - C15 product distribution, weight \% } \\
\hline $\mathrm{C} 1$ & 21.71 & 25.28 & 28.12 & 29.22 & 28.49 & 28.39 \\
\hline $\mathrm{C} 2$ & 13.50 & 4.64 & 5.17 & 5.33 & 5.22 & 5.16 \\
\hline C3 & 10.56 & 11.58 & 12.46 & 12.54 & 12.24 & 12.02 \\
\hline $\mathrm{C} 4$ & 10.83 & 11.65 & 12.25 & 12.09 & 11.88 & 11.67 \\
\hline C5 & 10.39 & 11.16 & 11.33 & 11.28 & 11.31 & 11.20 \\
\hline C6 & 7.57 & 8.62 & 7.60 & 6.99 & 8.20 & 8.30 \\
\hline C7 & 7.78 & 8.32 & 7.07 & 7.05 & 7.05 & 7.06 \\
\hline $\mathrm{C} 8$ & 5.54 & 5.88 & 5.13 & 5.04 & 5.02 & 5.04 \\
\hline $\mathrm{C} 9$ & 3.93 & 4.18 & 3.70 & 3.59 & 3.59 & 3.64 \\
\hline C10 & 2.82 & 3.01 & 2.66 & 2.58 & 2.60 & 2.65 \\
\hline C11 & 2.02 & 2.04 & 1.78 & 1.72 & 1.75 & 1.77 \\
\hline $\mathrm{C} 12$ & 1.47 & 1.49 & 1.22 & 1.15 & 1.19 & 1.31 \\
\hline C13 & 1.03 & 1.13 & 0.81 & 0.63 & 0.79 & 0.94 \\
\hline C14 & 0.46 & 0.69 & 0.38 & 0.34 & 0.37 & 0.62 \\
\hline C15 & 0.39 & 0.33 & 0.32 & 0.43 & 0.30 & 0.22 \\
\hline alpha chain growth probability & 0.64 & 0.63 & 0.63 & 0.65 & 0.62 & 0.61 \\
\hline \multicolumn{7}{|c|}{ C1 - C50 estimated total product distribution, weight \% } \\
\hline $\mathrm{C} 1$ & 21.7 & 25.5 & 28.1 & 28.7 & 28.5 & 28.8 \\
\hline $\mathrm{C} 2-\mathrm{C} 4$ & 34.8 & 28.1 & 29.8 & 29.5 & 29.3 & 29.3 \\
\hline C5 - C12 & 40.9 & 44.2 & 40.0 & 39.0 & 40.2 & 40.4 \\
\hline $\mathrm{C} 13-\mathrm{C} 50$ & 2.6 & 2.2 & 2.2 & 2.8 & 2.0 & 1.5 \\
\hline CO conversion, $\%$ & 5.6 & 4.9 & 3.7 & 2.8 & 3.6 & 3.9 \\
\hline rate, g CH2/g cat/hr & 0.33 & 0.28 & 0.21 & 0.16 & 0.21 & 0.23 \\
\hline $\mathrm{CO} 2$ formation, $\%$ & 0.3 & 0.3 & 0.4 & 0.4 & 0.3 & 0.2 \\
\hline
\end{tabular}


Time-on-Stream Plot for Co.004 - Run \#7

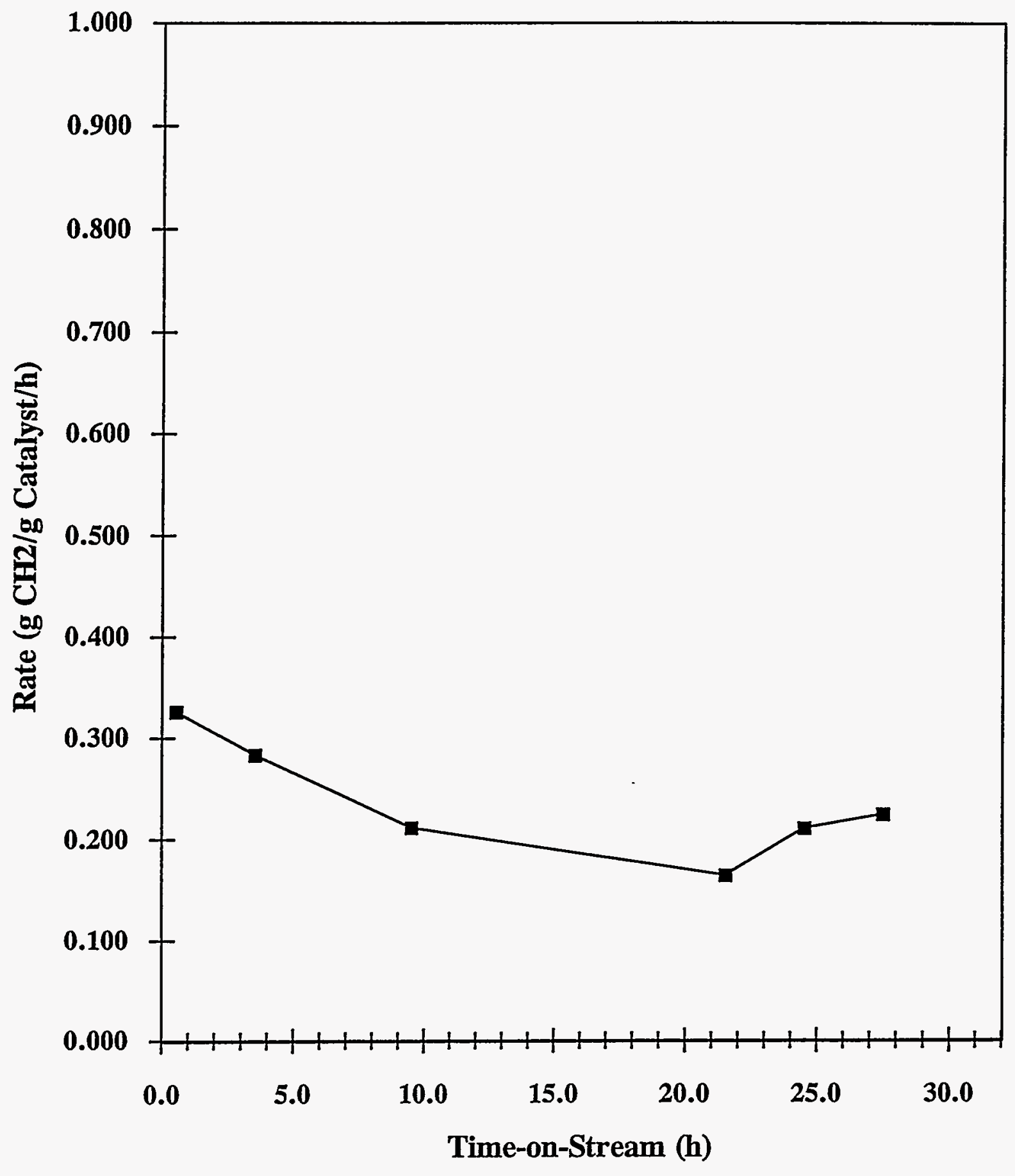




\section{Schulz-Flory Plot for Co.004 - Run \#7 \\ Time on Stream (hrs)}

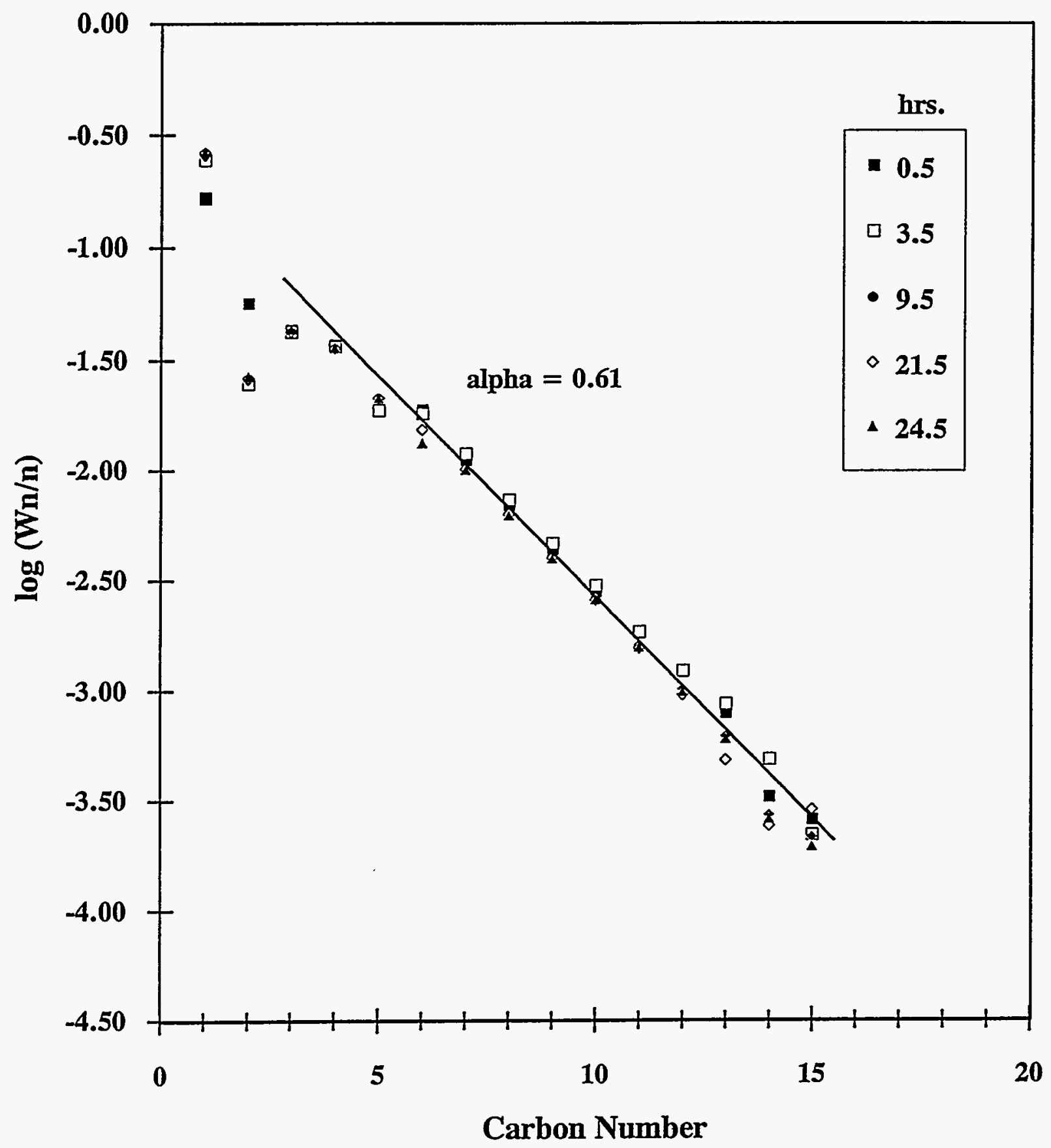




\section{Co.004 - Run \#8}

\begin{tabular}{|c|c|c|c|}
\hline Co wt\% & NM wt \% & Promotor wt\% & Support \\
\hline 20 & Ru $\quad 0.43$ & $\mathrm{La} 2 \mathrm{O3} 1.00$ & Al203 \\
\hline
\end{tabular}

\section{SUMMARY REACTION DATA*}

Reaction Conditions:

$$
\begin{array}{ll}
\mathrm{P}=1.0 \mathrm{~atm} & \mathrm{CO} 2(\mathrm{~g} / \mathrm{g} \mathrm{cat} / \mathrm{hr})=0.028 \\
\mathrm{~T}=220^{\circ} \mathrm{C} & \mathrm{CO} 2(\% \text { of CO})=0.1 \\
\mathrm{H} 2 / \mathrm{CO}=2 & \mathrm{O} / \mathrm{P}=2.13 \\
\text { weight of catalyst }=0.164 \mathrm{~g} & \\
\text { WHSV }=15.661 / \mathrm{hr}
\end{array}
$$

WHSV $=15.661 / \mathrm{hr}$

time on stream $=24.5 \mathrm{hrs}$

\begin{tabular}{|l|c|}
\hline \hline CO conversion (\%) & 5.8 \\
\hline rate (g CH2/g cat/hr) & 0.40 \\
\hline alpha & 0.66 \\
\hline $\mathrm{C} 1$ (wt\%) & 27.2 \\
\hline $\mathrm{C} 2-\mathrm{C} 4(\mathrm{wt} \%)$ & 27.4 \\
\hline $\mathrm{C} 5-\mathrm{C} 12(\mathrm{wt} \%)$ & 41.6 \\
\hline $\mathrm{C} 13+(\mathrm{wt} \%)$ & 3.8 \\
\hline
\end{tabular}

* Catalyst calcined in helium 


\section{Performance of Co.004}

\section{Dates: 11/11/94 - 11/12/94 Run \#8}

flow rate $=90.0 \mathrm{cc} / \mathrm{min}$, loading $=0.2 \mathrm{~g}, \mathrm{WHSV}=15.7 \mathrm{1} / \mathrm{hr}, \mathrm{H} 2 / \mathrm{CO}$ ratio in feed $=2$

\begin{tabular}{|c|c|c|c|c|c|c|}
\hline time on stream, hr & 0.5 & 3.5 & 6.5 & 9.5 & 12.5 & 15.5 \\
\hline reaction temperature, ${ }^{\circ} \mathrm{C}$ & 220 & 220 & 220 & 220 & 220 & 220 \\
\hline pressure, atm & 1.0 & 1.0 & 1.0 & 1.0 & 1.0 & 1.0 \\
\hline flow, cc/min & 90.0 & 90.0 & 90.0 & 90.0 & 90.0 & 90.0 \\
\hline \multicolumn{7}{|c|}{ C1 - C15 product distribution, weight $\%$} \\
\hline $\mathrm{C} 1$ & 24.37 & 25.97 & 26.59 & 27.18 & 27.53 & 27.59 \\
\hline $\mathrm{C} 2$ & 4.41 & 4.63 & 4.69 & 4.76 & 4.82 & 4.82 \\
\hline $\mathrm{C} 3$ & 11.68 & 11.88 & 11.81 & 11.86 & 11.91 & 11.89 \\
\hline $\mathrm{C} 4$ & 11.92 & 12.16 & 11.77 & 11.75 & 11.73 & 11.69 \\
\hline $\mathrm{C} 5$ & 11.41 & 11.31 & 11.02 & 10.95 & 11.01 & 10.99 \\
\hline C6 & 8.99 & 7.89 & 8.99 & 8.79 & 8.52 & 8.60 \\
\hline $\mathrm{C7}$ & 8.37 & 8.16 & 7.84 & 7.78 & 7.70 & 7.68 \\
\hline $\mathrm{C} 8$ & 5.82 & 5.70 & 5.48 & 5.42 & 5.37 & 5.37 \\
\hline C9 & 4.12 & 3.97 & 3.83 & 3.79 & 3.75 & 3.75 \\
\hline C10 & 2.92 & 2.79 & 2.71 & 2.69 & 2.64 & 2.66 \\
\hline C11 & 2.10 & 1.96 & 1.89 & 1.81 & 1.82 & 1.72 \\
\hline C12 & 1.41 & 1.33 & 1.29 & 1.18 & 1.25 & 1.24 \\
\hline $\mathrm{C} 13$ & 0.92 & 0.82 & 0.82 & 0.79 & 0.76 & 0.80 \\
\hline C14 & 0.79 & 0.77 & 0.65 & 0.70 & 0.64 & 0.65 \\
\hline C15 & 0.76 & 0.67 & 0.61 & 0.58 & 0.56 & 0.57 \\
\hline alpha chain growth probability & 0.68 & 0.67 & 0.66 & 0.66 & 0.66 & 0.66 \\
\hline \multicolumn{7}{|c|}{ C1 - C50 estimated total product distribution, weight \% } \\
\hline $\mathrm{C} 1$ & 23.5 & 25.2 & 25.9 & 26.5 & 26.9 & 26.9 \\
\hline $\mathrm{C} 2-\mathrm{C} 4$ & 27.0 & 27.8 & 27.5 & 27.6 & 27.8 & 27.7 \\
\hline $\mathrm{C} 5-\mathrm{C} 12$ & 44.6 & 42.7 & 42.7 & 42.1 & 41.7 & 41.7 \\
\hline $\mathrm{C} 13-\mathrm{C} 50$ & 4.9 & 4.4 & 4.0 & 3.8 & 3.7 & 3.7 \\
\hline CO conversion, \% & 8.1 & 7.3 & 7.1 & 6.8 & 6.5 & 6.3 \\
\hline rate, $\mathrm{g} \mathrm{CH} 2 / \mathrm{g} \mathrm{cat} / \mathrm{hr}$ & 0.56 & 0.50 & 0.49 & 0.47 & 0.44 & 0.43 \\
\hline $\mathrm{CO} 2$ formation, $\%$ & 0.2 & 0.2 & 0.1 & 0.2 & 0.1 & 0.1 \\
\hline
\end{tabular}




\section{Performance of Co.004}

\section{Dates: 11/11/94 - 11/12/94 Run \#8}

flow rate $=90.0 \mathrm{cc} / \mathrm{min}$, loading $=0.2 \mathrm{~g}, \mathrm{WHSV}=15.7 \mathrm{1} / \mathrm{hr}, \mathrm{H} 2 / \mathrm{CO}$ ratio in feed $=2$

\begin{tabular}{|c|c|c|c|}
\hline time on stream, hr & 18.5 & 21.5 & 24.5 \\
\hline reaction temperature, ${ }^{\circ} \mathrm{C}$ & 220 & 220 & 220 \\
\hline pressure, atm & 1.0 & 1.0 & 1.0 \\
\hline flow, $\mathrm{cc} / \mathrm{min}$ & 90.0 & 90.0 & 90.0 \\
\hline \multicolumn{4}{|c|}{$\mathrm{C} 1$ - C15 product distribution, weight \% } \\
\hline $\mathrm{Cl}$ & 27.62 & 27.80 & 27.97 \\
\hline $\mathrm{C} 2$ & 4.82 & 4.84 & 4.86 \\
\hline C3 & 11.82 & 11.87 & 11.86 \\
\hline $\mathrm{C} 4$ & 11.63 & 11.58 & 11.49 \\
\hline C5 & 10.99 & 11.00 & 10.97 \\
\hline C6 & 8.70 & 8.78 & 8.75 \\
\hline $\mathrm{C7}$ & 7.67 & 7.68 & 7.58 \\
\hline $\mathrm{C} 8$ & 5.39 & 5.33 & 5.33 \\
\hline $\mathrm{Cg}$ & 3.75 & 3.72 & 3.72 \\
\hline $\mathrm{C} 10$ & 2.66 & 2.62 & 2.62 \\
\hline $\mathrm{C} 11$ & 1.72 & 1.72 & 1.73 \\
\hline $\mathrm{C} 12$ & 1.21 & 1.09 & 1.12 \\
\hline $\mathrm{C} 13$ & 0.77 & 0.75 & 0.76 \\
\hline $\mathrm{C} 14$ & 0.67 & 0.66 & 0.66 \\
\hline $\mathrm{C} 15$ & 0.59 & 0.56 & 0.58 \\
\hline alpha chain growth probability & 0.66 & 0.66 & 0.66 \\
\hline \multicolumn{4}{|c|}{ C1 - C50 estimated total product distribution, weight $\%$} \\
\hline C1 & 26.9 & 27.0 & 27.2 \\
\hline $\mathrm{C} 2-\mathrm{C} 4$ & 27.5 & 27.5 & 27.4 \\
\hline C5 - C12 & 41.8 & 41.7 & 41.6 \\
\hline $\mathrm{C} 13-\mathrm{C} 50$ & 3.8 & 3.7 & 3.8 \\
\hline CO conversion, $\%$ & 6.1 & 6.0 & 5.8 \\
\hline rate, g CH $2 / g$ cat $/ \mathrm{hr}$ & 0.42 & 0.41 & 0.40 \\
\hline $\mathrm{CO} 2$ formation, \% & 0.1 & 0.2 & 0.1 \\
\hline
\end{tabular}


Time-on-Stream Plot for Co.004 - Run \#8

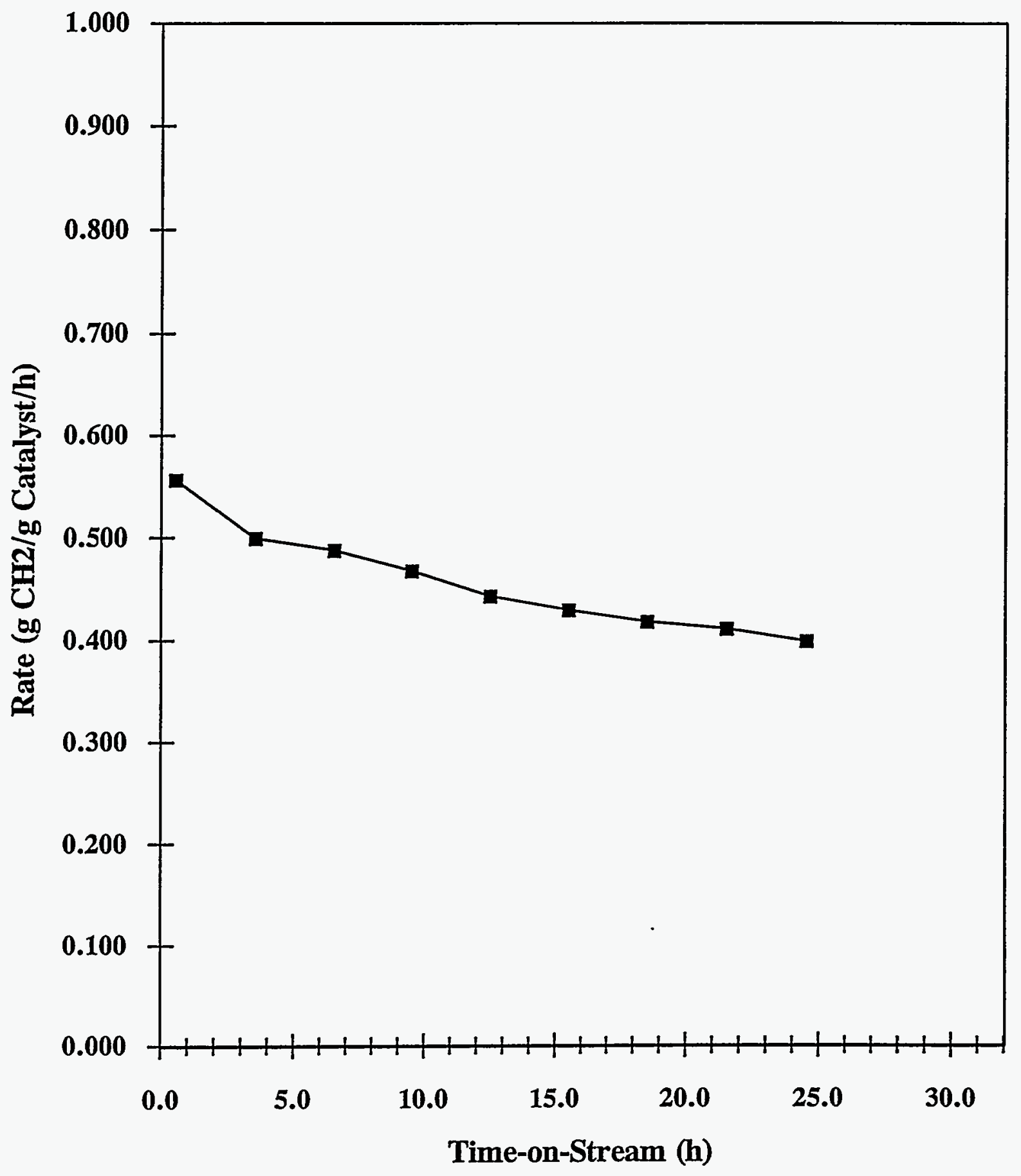


Schulz-Flory Plot for Co.004 - Run \#8

Time on Stream (hrs)

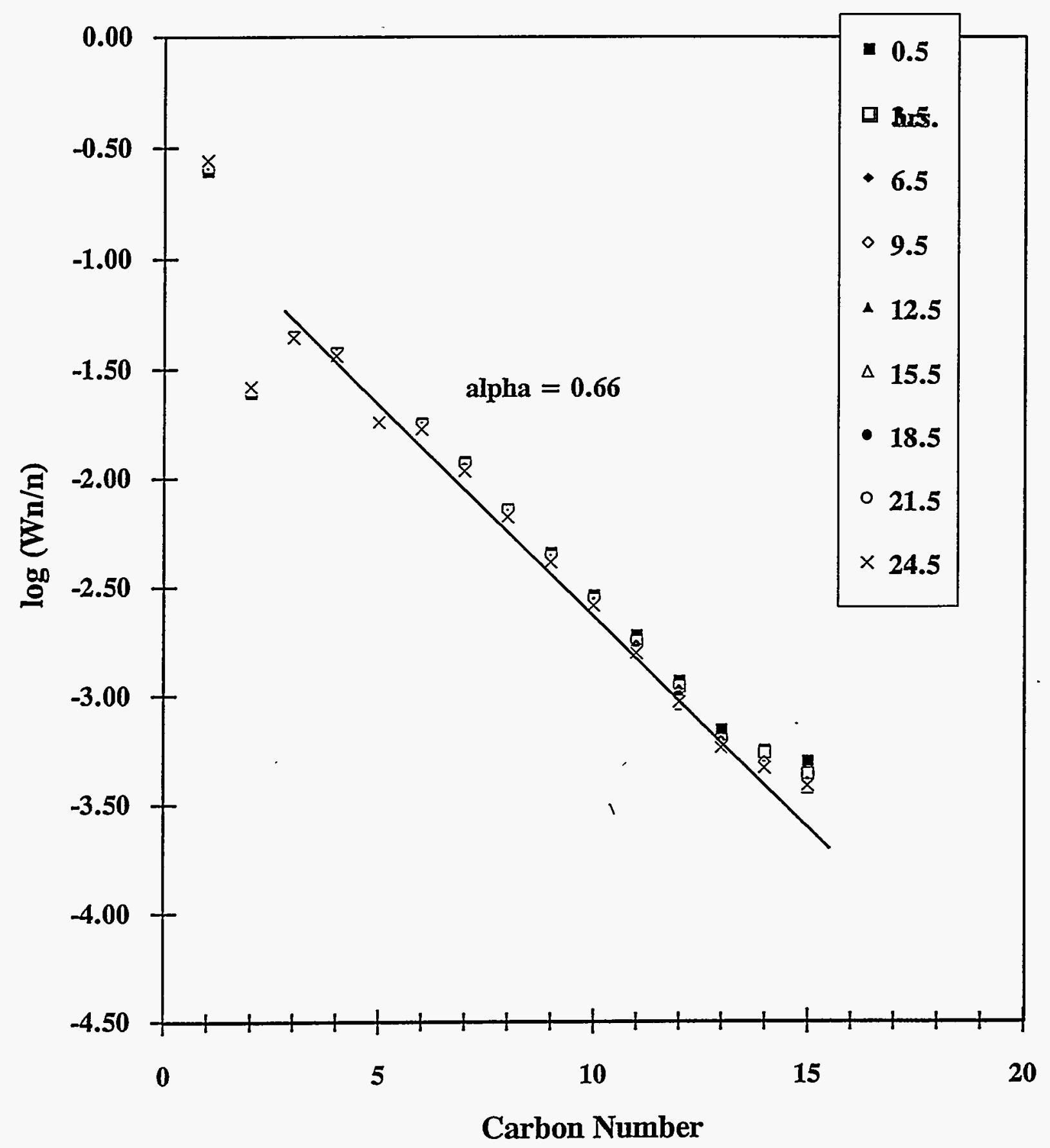

B13 


\section{Co.015 - Run \#3}

\begin{tabular}{|c|c|c|c|}
\hline Co wt\% & $\mathrm{NM}$ wt \% & Promotor wt\% & Support \\
\hline 20 & $\begin{array}{ll}\mathrm{Ru} & \mathbf{0 . 4 3}\end{array}$ & $\mathrm{La2O3} 1.00$ & $\mathrm{Al} 2 \mathrm{O} 3$ \\
\hline
\end{tabular}

\section{SUMMARY REACTION DATA}

Reaction Conditions:

$\mathrm{P}=1.0 \mathrm{~atm}$

$\mathrm{T}=220^{\circ} \mathrm{C}$

$\mathrm{H} 2 / \mathrm{CO}=2$

weight of catalyst $=0.200 \mathrm{~g}$

WHSV $=12.841 / \mathrm{hr}$

time on stream $=24.5 \mathrm{hrs}$
$\mathrm{CO} 2(\mathrm{~g} / \mathrm{g} \mathrm{cat} / \mathrm{hr})=0.031$

$\mathrm{CO} 2(\%$ of $\mathrm{CO})=0.2$

$\mathrm{O} / \mathrm{P}=1.62$

\begin{tabular}{||l|c|}
\hline CO conversion (\%) & 6.7 \\
\hline rate (g CH2/g cat/hr) & 0.38 \\
\hline alpha & 0.63 \\
\hline C1 (wt\%) & 28.2 \\
\hline C2 - C4 (wt\%) & 29.2 \\
\hline C5 - C12 (wt\%) & 40.3 \\
\hline C13 + (wt\%) & 2.3 \\
\hline
\end{tabular}

* Catalyst reduced without any calcination 


\section{Performance of Co.015}

Dates: 10/05/94 - 10/06/94 Run \#3

flow rate $=90.0 \mathrm{cc} / \mathrm{min}$, loading $=0.2 \mathrm{~g}, \mathrm{WHSV}=12.81 / \mathrm{hr}, \mathrm{H} 2 / \mathrm{CO}$ ratio in feed $=2$

\begin{tabular}{|c|c|c|c|c|c|c|}
\hline time on stream, hr & 0.5 & 3.5 & 6.5 & 9.5 & 12.5 & 21.5 \\
\hline reaction temperature, ${ }^{\circ} \mathrm{C}$ & 220 & 220 & 220 & 220 & 220 & 220 \\
\hline pressure, atm & 1.0 & 1.0 & 1.0 & 1.0 & 1.0 & 1.0 \\
\hline flow, cc/min & 90.0 & 90.0 & 90.0 & 90.0 & 90.0 & 90.0 \\
\hline \multicolumn{7}{|c|}{ C1 - C15 product distribution, weight $\%$} \\
\hline $\mathrm{C} 1$ & 28.23 & 28.03 & 30.00 & 28.81 & 26.13 & 27.79 \\
\hline $\mathrm{C} 2$ & 5.16 & 5.06 & 5.41 & 5.16 & 4.67 & 4.96 \\
\hline C3 & 13.62 & 12.86 & 13.62 & 12.90 & 11.59 & 12.18 \\
\hline $\mathrm{C} 4$ & 13.25 & 12.67 & 13.31 & 12.82 & 11.54 & 12.13 \\
\hline C5 & 11.26 & 11.41 & 11.64 & 11.94 & 10.69 & 11.46 \\
\hline C6 & 6.69 & 8.13 & 7.15 & 8.88 & 7.95 & 8.60 \\
\hline $\mathrm{C7}$ & 7.77 & 7.79 & 6.77 & 6.92 & 10.10 & 7.94 \\
\hline $\mathrm{C} 8$ & 5.08 & 5.13 & 4.45 & 4.63 & 6.38 & 5.35 \\
\hline $\mathrm{Cg}$ & 3.34 & 3.35 & 2.89 & 3.00 & 3.99 & 3.51 \\
\hline $\mathrm{C} 10$ & 2.07 & 2.09 & 1.83 & 1.91 & 2.65 & 2.30 \\
\hline $\mathrm{C} 11$ & 1.46 & 1.41 & 1.20 & 1.27 & 1.71 & 1.57 \\
\hline $\mathrm{C} 12$ & 0.87 & 0.78 & 0.73 & 0.68 & 1.10 & 0.86 \\
\hline C13 & 0.64 & 0.56 & 0.43 & 0.44 & 0.63 & 0.57 \\
\hline C14 & 0.38 & 0.42 & 0.34 & 0.35 & 0.48 & 0.45 \\
\hline $\mathrm{C} 15$ & 0.18 & 0.31 & 0.24 & 0.28 & 0.38 & 0.35 \\
\hline alpha chain growth probability & 0.59 & 0.62 & 0.61 & 0.61 & 0.63 & 0.63 \\
\hline \multicolumn{7}{|c|}{ C1 - C50 estimated total product distribution, weight \% } \\
\hline $\mathrm{C} 1$ & 28.4 & 27.7 & 29.7 & 28.5 & 25.8 & 27.4 \\
\hline $\mathrm{C} 2-\mathrm{C} 4$ & 32.2 & 30.2 & 32.1 & 30.5 & 27.5 & 28.9 \\
\hline $\mathrm{C} 5-\mathrm{C} 12$ & 38.2 & 40.0 & 36.6 & 39.2 & 44.2 & 41.4 \\
\hline C13 - C50 & 1.2 & 2.1 & 1.6 & 1.8 & 2.5 & 2.3 \\
\hline CO conversion, \% & 6.5 & 8.0 & 6.7 & 7.0 & 7.6 & 6.6 \\
\hline rate, $\mathrm{g} \mathrm{CH} 2 / \mathrm{g} \mathrm{cat} / \mathrm{hr}$ & 0.37 & 0.45 & 0.37 & 0.39 & 0.42 & 0.37 \\
\hline $\mathrm{CO} 2$ formation, $\%$ & 0.3 & 0.2 & 0.2 & 0.1 & 0.1 & 0.1 \\
\hline
\end{tabular}




\section{Performance of Co.015}

Dates: 10/05/94 - 10/06/94 Run \#3

\begin{tabular}{lcc} 
flow rate $=90.0$ cc/min, loading $=0.2 \mathrm{~g}$, WHSV $=12.81 / \mathrm{hr}$, H2/CO ratio in feed $=2$ \\
\hline time on stream, hr & 24.5 & 27.5 \\
reaction temperature, ${ }^{\circ} \mathrm{C}$ & 220 & 220 \\
pressure, atm & 1.0 & 1.0 \\
flow, cc/min & 90.0 & 90.0 \\
\hline & & \\
C1 - C15 product distribution, weight \% & & \\
& & \\
C1 & 28.59 & 27.93 \\
& & \\
C2 & 5.07 & 4.97 \\
C3 & 12.34 & 12.11 \\
C4 & 12.17 & 12.03 \\
& & \\
C5 & 11.36 & 11.34 \\
C6 & 7.92 & 8.55 \\
C7 & 7.81 & 7.93 \\
C8 & 5.30 & 5.37 \\
C9 & 3.45 & 3.52 \\
C10 & 2.25 & 2.36 \\
C11 & 1.52 & 1.41 \\
C12 & 0.89 & 1.08 \\
C13 & 0.55 & 0.61 \\
C14 & 0.43 & 0.43 \\
C15 & 0.35 & 0.36 \\
& & \\
alpha chain growth probability & 0.63 & 0.63 \\
& & \\
\hline
\end{tabular}

C1 - C50 estimated total product distribution, weight \%

C1

$\mathrm{C} 2-\mathrm{C} 4$

C5 - $\mathrm{C} 12$

C13 - C50
$28.2 \quad 27.6$

$29.2 \quad 28.7$

$40.3 \quad 41.3$

$2.3 \quad 2.4$
Co conversion, $\%$ rate, $\mathrm{g} \mathrm{CH} 2 / \mathrm{g} \mathrm{cat} / \mathrm{hr}$

$\mathrm{CO} 2$ formation, $\%$

$\begin{array}{ll}6.7 & 6.4 \\ 0.38 & 0.36 \\ 0.2 & 0.1\end{array}$

$6.7 \quad 6.4$

$0.2 \quad 0.1$ 
Time-on-Stream Plot for Co.015 - Run \#3

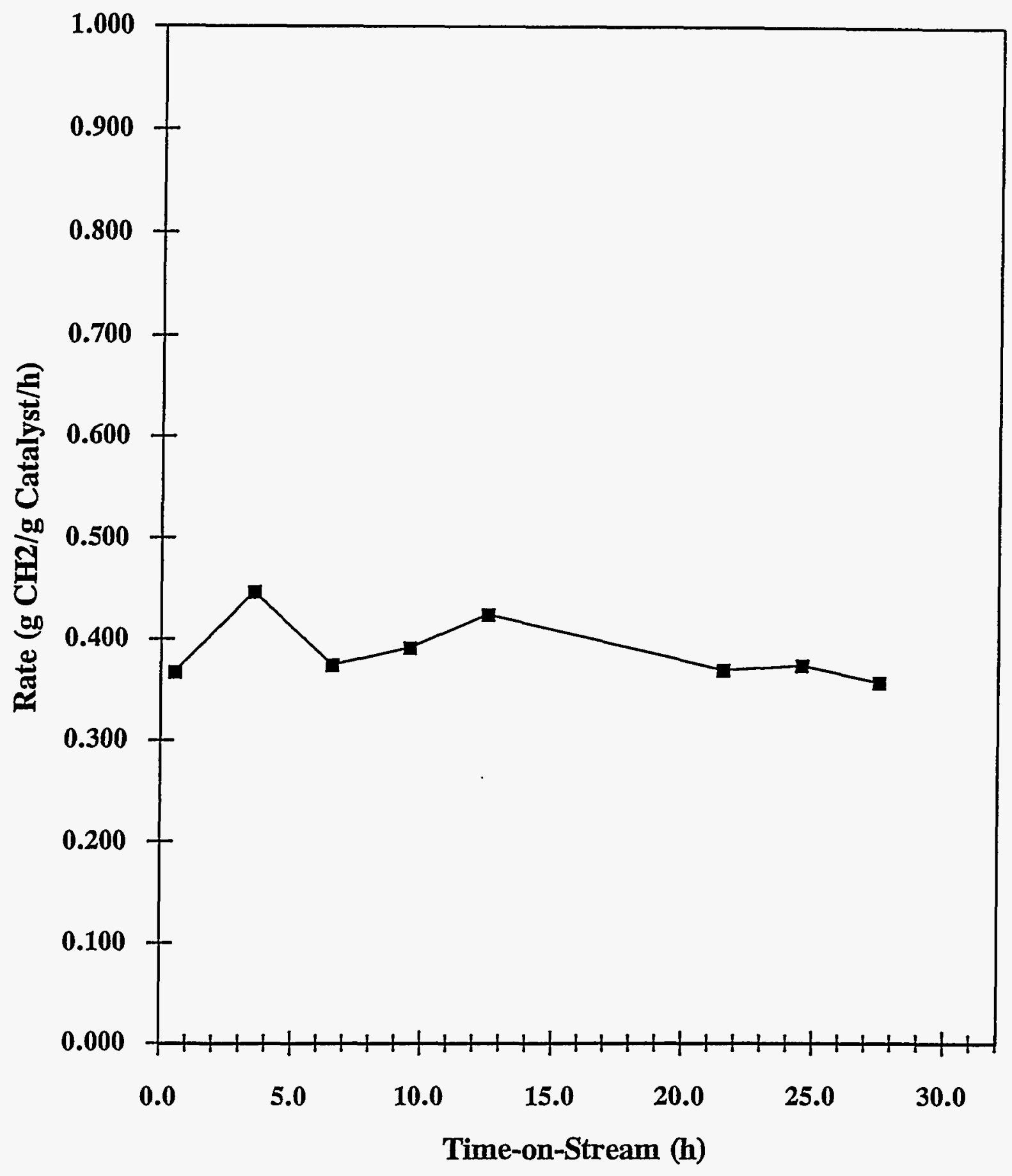


Schulz-Flory Plot for Co.015 - Run \#3

Time on Stream (hrs)

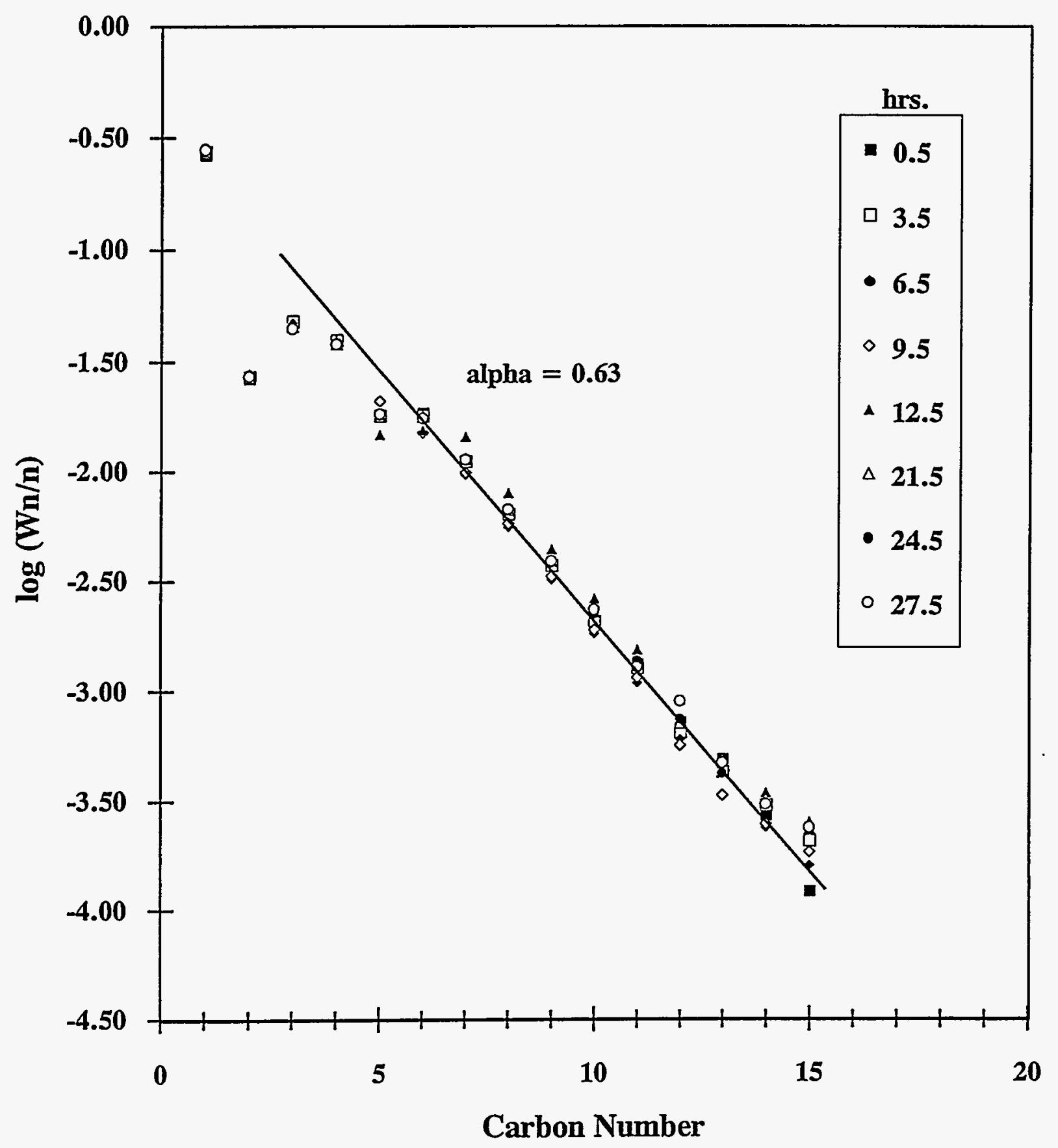




\section{Co.017 - Run \#3}

\begin{tabular}{|c|c|c|c|}
\hline Co wt\% & NM wt \% & Promotor wt\% & Support \\
\hline 20 & Ru $\quad 0.50$ & $\mathrm{La} 2 \mathrm{O} 31.00$ & $\mathrm{Al2O}$ \\
\hline
\end{tabular}

\section{SUMMARY REACTION DATA}

Reaction Conditions:

$$
\begin{array}{ll}
\mathrm{P}=1.0 \mathrm{~atm} & \mathrm{CO} 2(\mathrm{~g} / \mathrm{g} \mathrm{cat} / \mathrm{hr})=0.030 \\
\mathrm{~T}=220^{\circ} \mathrm{C} & \mathrm{CO} 2(\% \text { of } \mathrm{CO})=0.2 \\
\mathrm{H} 2 / \mathrm{CO}=2 & \mathrm{O} / \mathrm{P}=2.41 \\
\text { weight of catalyst }=0.201 \mathrm{~g} & \\
\text { WHSV }=12.811 / \mathrm{hr} & \\
\text { time on stream }=9.5 \mathrm{hrs} &
\end{array}
$$

\begin{tabular}{|l|c|}
\hline CO conversion (\%) & 4.9 \\
\hline rate (g CH2/g cat/hr) & 0.27 \\
\hline alpha & 0.67 \\
\hline $\mathrm{C} 1$ (wt\%) & 23.0 \\
\hline $\mathrm{C} 2-\mathrm{C} 4(\mathrm{wt} \%)$ & 26.1 \\
\hline $\mathrm{C} 5-\mathrm{C} 12(\mathrm{wt} \%)$ & 46.9 \\
\hline $\mathrm{C} 13+(w \mathrm{t} \%)$ & 4.0 \\
\hline
\end{tabular}

* Reaction data for only $10 \mathrm{hrs}$. on stream because of T serge after $10 \mathrm{hrs}$ 


\section{Performance of Co.017}

Dates: 10/03/94 - 10/04/94 Run \#3

flow rate $=90.0 \mathrm{cc} / \mathrm{min}$, loading $=0.2 \mathrm{~g}, \mathrm{WHSV}=12.81 / \mathrm{hr}, \mathrm{H} 2 / \mathrm{CO}$ ratio in feed $=2$

\begin{tabular}{|c|c|c|c|c|}
\hline time on stream, $\mathrm{hr}$ & 0.5 & 3.5 & 6.5 & 9.5 \\
\hline reaction temperature, ${ }^{\circ} \mathrm{C}$ & 220 & 220 & 220 & 220 \\
\hline pressure, atm & 1.0 & 1.0 & 1.0 & 1.0 \\
\hline flow, cc/min & 90.0 & 90.0 & 90.0 & 90.0 \\
\hline \multicolumn{5}{|c|}{$\mathrm{C} 1-\mathrm{C} 15$ product distribution, weight $\%$} \\
\hline $\mathrm{Cl}$ & 21.38 & 22.13 & 20.46 & 23.34 \\
\hline $\mathrm{C} 2$ & 4.07 & 4.14 & 11.99 & 4.39 \\
\hline $\mathrm{C} 3$ & 10.97 & 10.56 & 9.43 & 10.65 \\
\hline $\mathrm{C} 4$ & 11.92 & 11.48 & 10.33 & 11.52 \\
\hline C5 & 12.09 & 11.73 & 10.62 & 11.89 \\
\hline C6 & 9.71 & 9.57 & 8.85 & 9.38 \\
\hline C7 & 9.24 & 9.20 & 8.50 & 8.51 \\
\hline $\mathrm{C} 8$ & 6.57 & 6.70 & 6.19 & 6.26 \\
\hline C9 & 4.56 & 4.69 & 4.42 & 4.54 \\
\hline $\mathrm{C} 10$ & 3.10 & 3.21 & 3.14 & 3.29 \\
\hline $\mathrm{C} 11$ & 2.25 & 2.32 & 2.21 & 2.29 \\
\hline $\mathrm{C} 12$ & 1.52 & 1.57 & 1.41 & 1.50 \\
\hline C13 & 1.22 & 1.08 & 0.99 & 1.05 \\
\hline C14 & 0.83 & 0.93 & 0.85 & 0.81 \\
\hline $\mathrm{C} 15$ & 0.56 & 0.69 & 0.61 & 0.60 \\
\hline alpha chain growth probability & 0.66 & 0.68 & 0.67 & 0.67 \\
\hline \multicolumn{5}{|c|}{ C1 - C50 estimated total product distribution, weight \% } \\
\hline $\mathrm{C} 1$ & 21.2 & 21.6 & 20.1 & 23.0 \\
\hline $\mathrm{C} 2-\mathrm{C} 4$ & 26.7 & 25.6 & 31.1 & 26.1 \\
\hline $\mathrm{C} 5-\mathrm{C} 12$ & 48.4 & 48.1 & 44.8 & 46.9 \\
\hline $\mathrm{C} 13-\mathrm{C} 50$ & 3.8 & 4.7 & 4.0 & 4.0 \\
\hline CO conversion, $\%$ & 7.0 & 6.5 & 6.5 & 4.9 \\
\hline rate, $\mathrm{g} \mathrm{CH} 2 / \mathrm{g} \mathrm{cat} / \mathrm{hr}$ & 0.39 & 0.36 & 0.36 & 0.27 \\
\hline $\mathrm{CO} 2$ formation, $\%$ & 0.1 & 0.2 & 0.1 & 0.2 \\
\hline
\end{tabular}


Time-on-Stream Plot for Co.017 - Run \#3

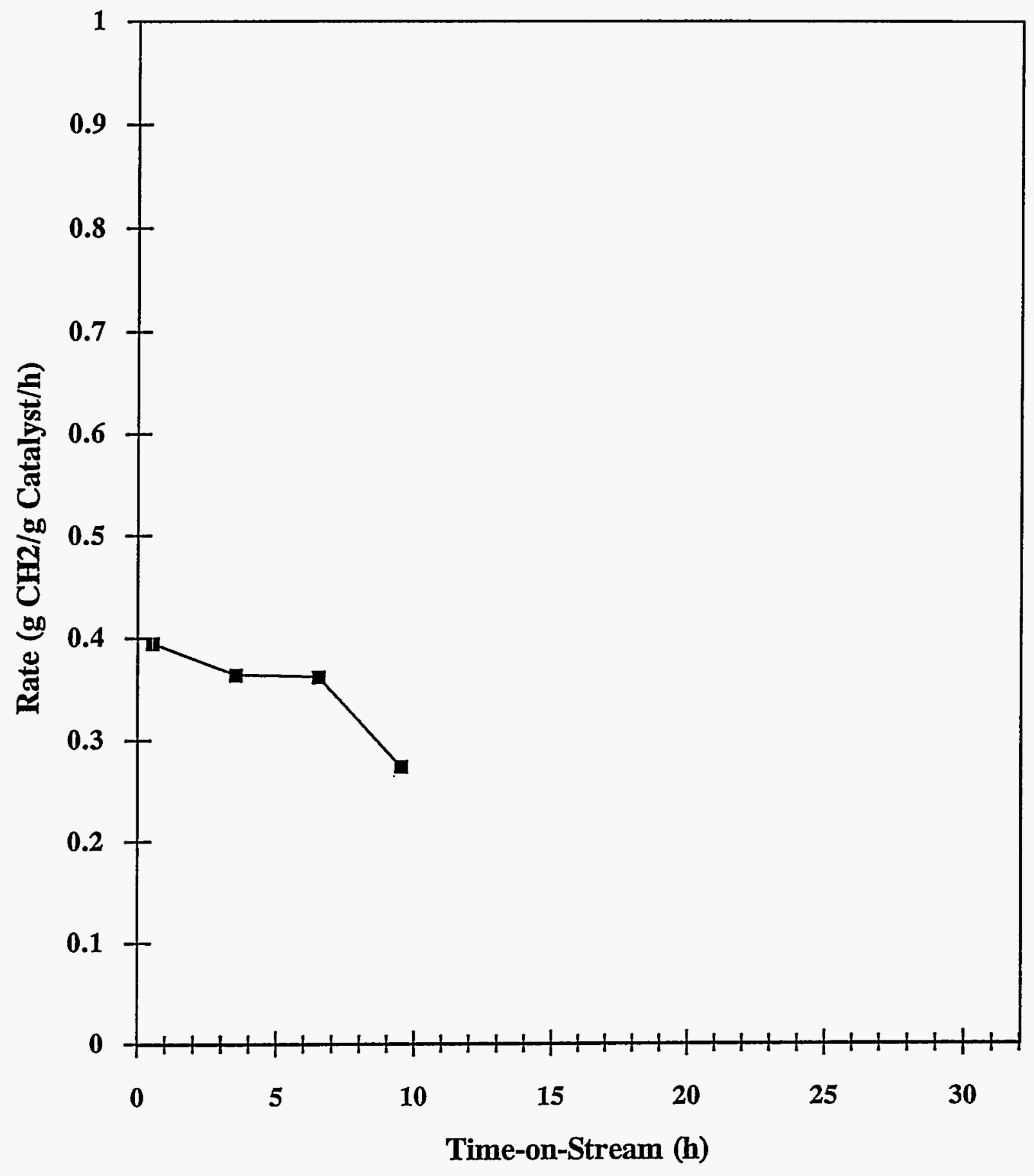


Schulz-Flory Plot for Co.017 - Run \#3

Time on Stream (hrs)

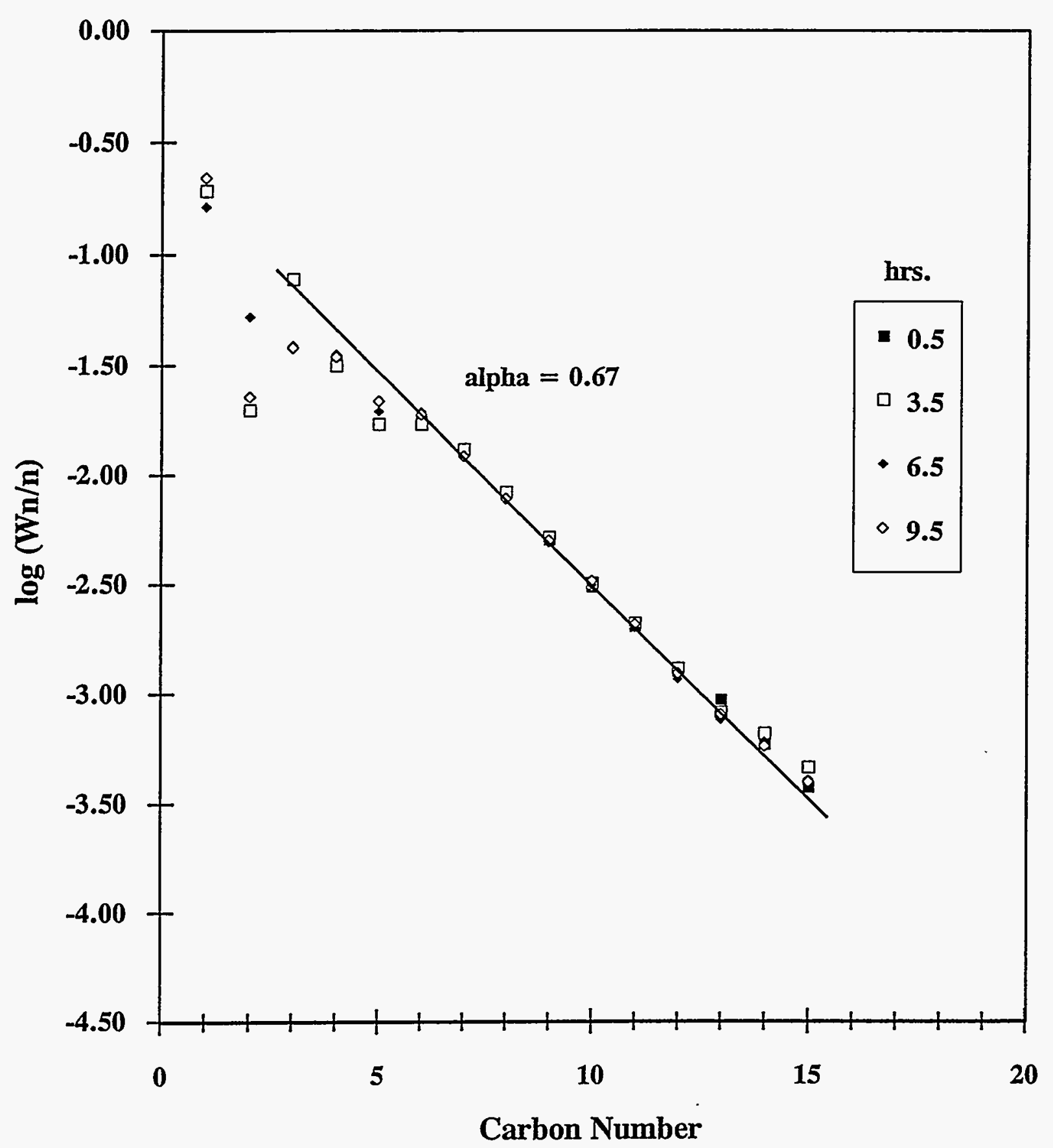




\section{Co.044 - Run \#1}

\begin{tabular}{|c||cc|c|c||c||}
\hline Co wt\% & NM wt \% & \multicolumn{3}{|c|}{ Promotor wt\% } & Support \\
\hline 20 & & & $\mathrm{Zr}$ & 15.00 & $\mathrm{SiO} 2$ \\
\hline
\end{tabular}

\section{SUMMARY REACTION DATA}

Reaction Conditions:

$$
\begin{array}{ll}
\mathrm{P}=1.0 \mathrm{~atm} & \mathrm{CO} 2(\mathrm{~g} / \mathrm{g} \mathrm{cat} / \mathrm{hr})=0.020 \\
\mathrm{~T}=220^{\circ} \mathrm{C} & \mathrm{CO} 2(\% \text { of } \mathrm{CO})=0.1 \\
\mathrm{H} 2 / \mathrm{CO}=2 & \mathrm{O} / \mathrm{P}=5.16 \\
\text { weight of catalyst }=0.198 \mathrm{~g} & \\
\text { WHSV }=13.011 / \mathrm{hr} & \\
\text { time on stream }=24.5 \mathrm{hrs} &
\end{array}
$$

\begin{tabular}{|l|c|}
\hline CO conversion (\%) & 3.1 \\
\hline rate (g CH2/g cat/hr) & 0.18 \\
\hline alpha & 0.73 \\
\hline C1 (wt\%) & 22.7 \\
\hline C2 - C4 (wt\%) & 23.6 \\
\hline C5 - C12 (wt\%) & 44.3 \\
\hline C13 + (wt\%) & 9.5 \\
\hline
\end{tabular}




\section{Performance of $\mathbf{C o . 0 4 4}$ \\ Dates: 11/30/94 - 12/01/94 Run \#1}

flow rate $=90.0 \mathrm{cc} / \mathrm{min}$, loading $=0.2 \mathrm{~g}, \mathrm{WHSV}=13.01 / \mathrm{hr}, \mathrm{H} 2 / \mathrm{CO}$ ratio in feed $=2$

\begin{tabular}{|c|c|c|c|c|c|c|}
\hline time on stream, hr & 0.5 & 3.5 & 6.5 & 9.5 & 12.5 & 15.5 \\
\hline reaction temperature, ${ }^{\circ} \mathrm{C}$ & 220 & 220 & 220 & 220 & 220 & 220 \\
\hline pressure, atm & 1.0 & 1.0 & 1.0 & 1.0 & 1.0 & 1.0 \\
\hline flow, cc/min & 90.0 & 90.0 & 90.0 & 90.0 & 90.0 & 90.0 \\
\hline \multicolumn{7}{|c|}{ C1 - C15 product distribution, weight \% } \\
\hline $\mathrm{C} 1$ & 20.65 & 23.18 & 21.94 & 22.79 & 23.46 & 24.04 \\
\hline $\mathrm{C} 2$ & 4.60 & 5.06 & 4.74 & 4.85 & 4.94 & 5.04 \\
\hline C3 & 11.14 & 11.28 & 10.10 & 10.00 & 10.04 & 10.12 \\
\hline $\mathrm{C} 4$ & 11.79 & 11.90 & 10.47 & 10.50 & 10.40 & 10.43 \\
\hline C5 & 12.03 & 12.34 & 11.05 & 11.04 & 10.97 & 10.98 \\
\hline C6 & 8.55 & 10.26 & 9.92 & 9.85 & 9.49 & 8.58 \\
\hline $\mathrm{C7}$ & 8.59 & 0.03 & 8.22 & 8.02 & 7.90 & 7.95 \\
\hline $\mathrm{C} 8$ & 6.37 & 6.71 & 6.16 & 6.02 & 6.02 & 6.05 \\
\hline C9 & 4.82 & 5.08 & 4.61 & 4.54 & 4.54 & 4.57 \\
\hline $\mathrm{C} 10$ & 3.62 & 3.84 & 3.60 & 3.53 & 3.57 & 3.54 \\
\hline $\mathrm{C} 11$ & 2.66 & 2.82 & 2.56 & 2.56 & 2.58 & 2.57 \\
\hline $\mathrm{C} 12$ & 1.84 & 2.11 & 1.89 & 2.05 & 1.95 & 1.88 \\
\hline $\mathrm{C} 13$ & 1.29 & 1.76 & 1.60 & 1.48 & 1.51 & 1.55 \\
\hline $\mathrm{C} 14$ & 1.10 & 1.75 & 1.56 & 1.43 & 1.35 & 1.40 \\
\hline $\mathrm{C} 15$ & 0.95 & 1.88 & 1.59 & 1.32 & 1.26 & 1.30 \\
\hline alpha chain growth probability & 0.69 & 0.74 & 0.73 & 0.72 & 0.71 & 0.72 \\
\hline \multicolumn{7}{|c|}{ C1 - C50 estimated total product distribution, weight $\%$} \\
\hline $\mathrm{C} 1$ & 19.8 & 20.7 & 20.0 & 21.3 & 22.0 & 22.5 \\
\hline $\mathrm{C} 2-\mathrm{C} 4$ & 26.5 & 25.2 & 23.1 & 23.7 & 23.8 & 23.9 \\
\hline C5 - C12 & 47.4 & 41.7 & 46.4 & 46.2 & 46.0 & 44.9 \\
\hline $\mathrm{C} 13-\mathrm{C} 50$ & 6.3 & 12.3 & 10.5 & 8.8 & 8.3 & 8.7 \\
\hline CO conversion, \% & 4.7 & 4.1 & 4.0 & 3.7 & 3.6 & 3.4 \\
\hline rate, $\mathrm{g} \mathrm{CH} 2 / \mathrm{g}$ cat $/ \mathrm{hr}$ & 0.27 & 0.23 & 0.23 & 0.21 & 0.20 & 0.19 \\
\hline $\mathrm{CO} 2$ formation, $\%$ & 0.2 & 0.2 & 0.1 & 0.1 & 0.1 & 0.1 \\
\hline
\end{tabular}




\section{Performance of Co.044}

\section{Dates: 11/30/94 - 12/01/94 Run \#1}

\begin{tabular}{|c|c|c|c|}
\hline time on stream, $\mathrm{hr}$ & 18.5 & 21.5 & 24.5 \\
\hline reaction temperature, ${ }^{\circ} \mathrm{C}$ & 220 & 220 & 220 \\
\hline pressure, atm & 1.0 & 1.0 & 1.0 \\
\hline flow, cc/min & 90.0 & 90.0 & 90.0 \\
\hline \multicolumn{4}{|c|}{ C1 - C15 product distribution, weight \% } \\
\hline $\mathrm{C} 1$ & 24.24 & 24.37 & 24.51 \\
\hline $\mathrm{C} 2$ & 5.10 & 5.14 & 5.18 \\
\hline C3 & 10.18 & 10.16 & 10.15 \\
\hline $\mathrm{C} 4$ & 10.34 & 10.33 & 10.17 \\
\hline C5 & 10.82 & 10.85 & 10.81 \\
\hline C6 & 8.41 & 8.34 & 8.56 \\
\hline C7 & 8.04 & 8.01 & 7.96 \\
\hline $\mathrm{C} 8$ & 6.08 & 6.04 & 5.95 \\
\hline $\mathrm{C} 9$ & 4.55 & 4.56 & 4.49 \\
\hline $\mathrm{C} 10$ & 3.56 & 3.53 & 3.47 \\
\hline $\mathrm{C} 11$ & 2.54 & 2.51 & 2.47 \\
\hline $\mathrm{C} 12$ & 1.89 & 1.89 & 1.85 \\
\hline $\mathrm{C} 13$ & 1.48 & 1.50 & 1.50 \\
\hline $\mathrm{C} 14$ & 1.38 & 1.46 & 1.50 \\
\hline $\mathrm{C} 15$ & 1.38 & 1.33 & 1.42 \\
\hline alpha chain growth probability & 0.72 & 0.72 & 0.73 \\
\hline
\end{tabular}

C1 - C50 estimated total product distribution, weight \%

\begin{tabular}{lccc}
$\mathrm{C} 1$ & 22.5 & 22.7 & 22.7 \\
$\mathrm{C} 2-\mathrm{C} 4$ & 23.8 & 23.9 & 23.6 \\
$\mathrm{C} 5-\mathrm{C} 12$ & 44.5 & 44.5 & 44.3 \\
$\mathrm{C} 13-\mathrm{C} 50$ & 9.2 & 8.9 & 9.5 \\
& & & \\
\hline CO conversion, \% & 3.3 & 3.2 & 3.1 \\
rate, $\mathrm{g} \mathrm{CH} 2 / \mathrm{g} \mathrm{cat} / \mathrm{hr}$ & 0.19 & 0.18 & 0.18 \\
$\mathrm{CO}$ formation, \% & 0.1 & 0.1 & 0.1 \\
\hline
\end{tabular}


Time-on-Stream Plot for Co.044 - Run \#1

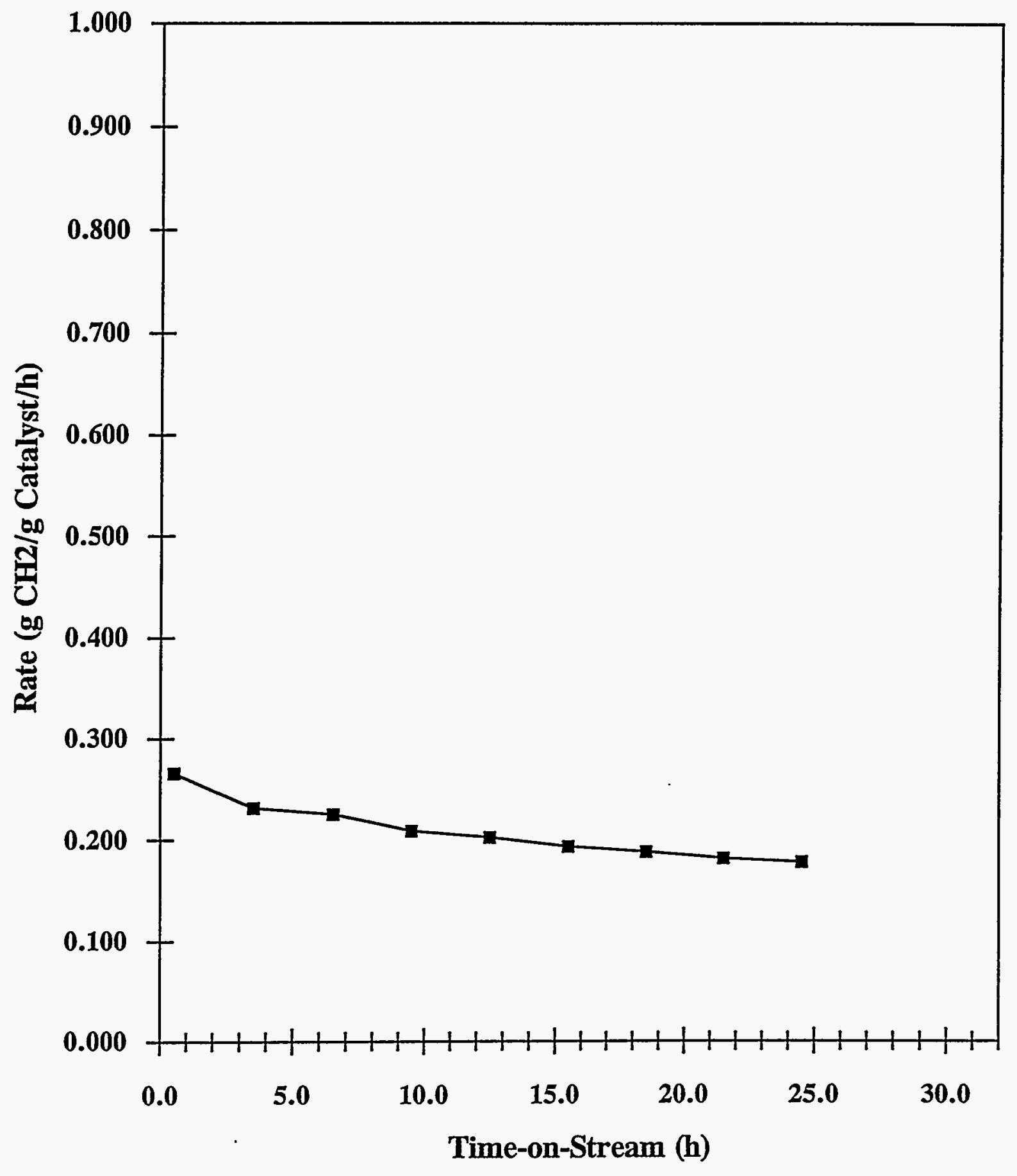


Schulz-Flory Plot for Co.044 - Run \#1

Time on Stream (hrs)

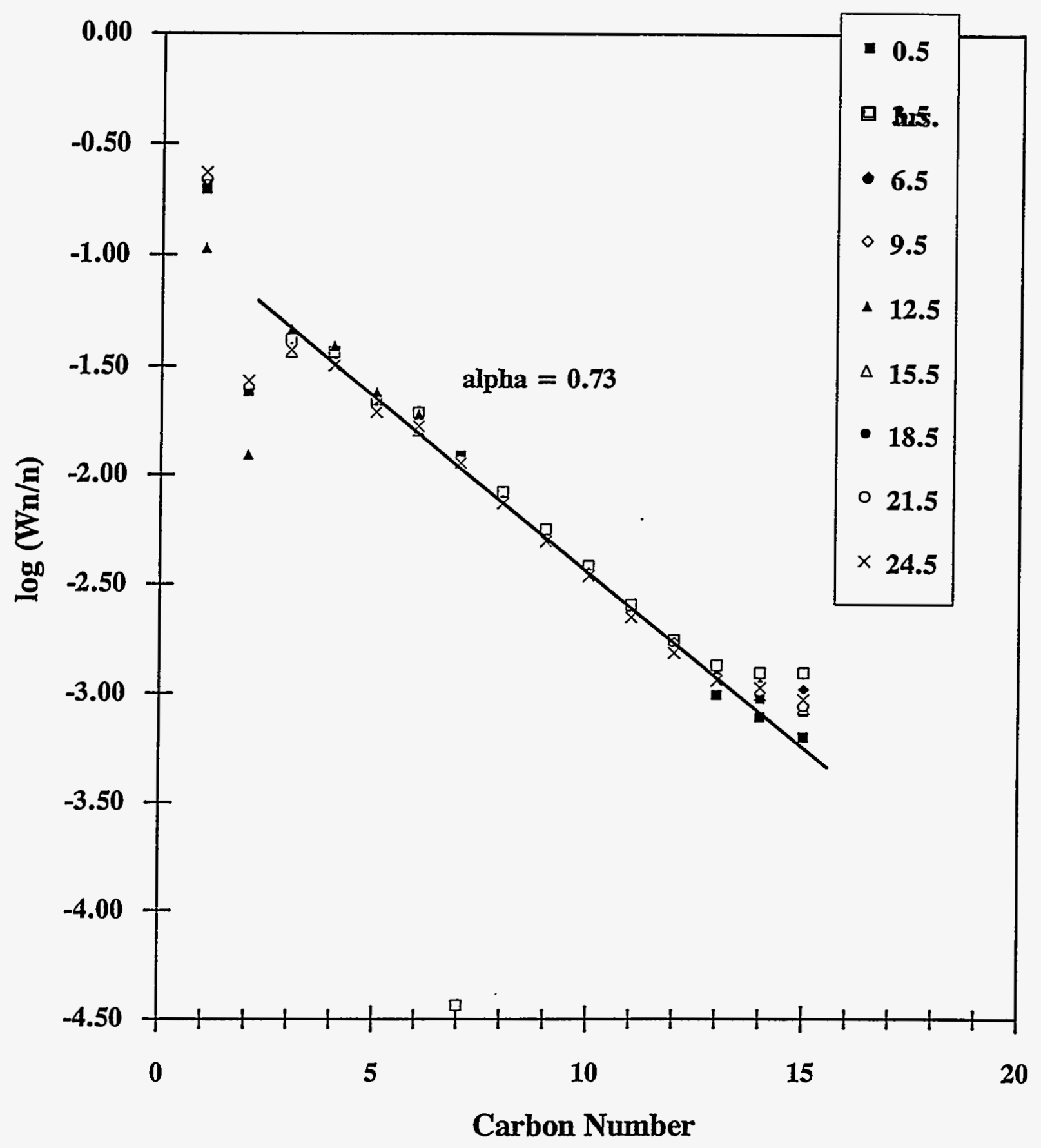




\section{Co.045 - Run \#1}

\begin{tabular}{|c|c|c|c|c|}
\hline Co wt\% & NM wt \% & Promotor w & & Support \\
\hline 20 & & & 4.00 & $\mathrm{SiO} 2$ \\
\hline
\end{tabular}

\section{SUMMARY REACTION DATA}

Reaction Conditions:

$$
\begin{array}{ll}
\mathrm{P}=1.0 \mathrm{~atm} & \mathrm{CO} 2(\mathrm{~g} / \mathrm{g} \mathrm{cat} / \mathrm{hr})=0.022 \\
\mathrm{~T}=220^{\circ} \mathrm{C} & \mathrm{CO} 2(\% \text { of CO})=0.1 \\
\mathrm{H} 2 / \mathrm{CO}=2 & \mathrm{O} / \mathrm{P}=6.89 \\
\text { weight of catalyst }=0.185 \mathrm{~g} & \\
\text { WHSV }=13.911 / \mathrm{hr} & \\
\text { time on stream }=21.5 \mathrm{hrs} &
\end{array}
$$

\begin{tabular}{||l|c||}
\hline \hline $\mathrm{CO}$ conversion (\%) & 2.6 \\
\hline rate (g CH2/g cat/hr) & 0.16 \\
\hline alpha & 0.73 \\
\hline $\mathrm{C} 1$ (wt\%) & 22.2 \\
\hline $\mathrm{C} 2-\mathrm{C} 4(\mathrm{wt} \%)$ & 22.8 \\
\hline $\mathrm{C} 5-\mathrm{C} 12(\mathrm{wt} \%)$ & 44.8 \\
\hline $\mathrm{C} 13+(\mathrm{wt} \%)$ & 10.2 \\
\hline
\end{tabular}




\title{
Performance of Co.045
}

\author{
Dates: 12/03/94 - 12/04/94 Run \#1
}

flow rate $=90.0 \mathrm{cc} / \mathrm{min}$, loading $=0.2 \mathrm{~g}, \mathrm{WHSV}=13.91 / \mathrm{hr}, \mathrm{H} 2 / \mathrm{CO}$ ratio in feed $=2$

\begin{tabular}{|c|c|c|c|c|c|c|}
\hline time on stream, $\mathbf{h r}$ & 0.5 & 3.5 & 6.5 & 9.5 & 12.0 & 15.5 \\
\hline reaction temperature, ${ }^{\circ} \mathrm{C}$ & 220 & 220 & 220 & 220 & 220 & 220 \\
\hline pressure, atm & 1.0 & 1.0 & 1.0 & 1.0 & 1.0 & 1.0 \\
\hline flow, cc/min & 90.0 & 90.0 & 90.0 & 90.0 & 90.0 & 90.0 \\
\hline \multicolumn{7}{|c|}{ C1 - C15 product distribution, weight \% } \\
\hline $\mathrm{C} 1$ & 19.46 & 21.01 & 22.19 & 22.81 & 24.13 & 23.54 \\
\hline $\mathrm{C} 2$ & 4.40 & 4.68 & 4.85 & 4.98 & 5.25 & 5.13 \\
\hline C3 & 9.90 & 9.46 & 9.50 & 9.53 & 13.96 & 9.55 \\
\hline $\mathrm{C} 4$ & 10.80 & 9.91 & 9.80 & 9.87 & 2.79 & 9.80 \\
\hline $\mathrm{C5}$ & 11.69 & 10.86 & 10.73 & 10.58 & 10.97 & 10.64 \\
\hline $\mathrm{C} 6$ & 9.90 & 9.36 & 8.60 & 8.75 & 9.24 & 8.89 \\
\hline $\mathrm{C7}$ & 8.91 & 8.59 & 8.77 & 8.46 & 8.42 & 8.10 \\
\hline $\mathrm{C} 8$ & 6.77 & 6.52 & 6.44 & 6.44 & 6.48 & 6.25 \\
\hline $\mathrm{C} 9$ & 5.23 & 5.00 & 5.04 & 4.95 & 4.95 & 4.82 \\
\hline C10 & 3.99 & 3.85 & 3.92 & 3.86 & 3.91 & 3.80 \\
\hline C11 & 2.91 & 2.82 & 2.80 & 2.80 & 2.85 & 2.75 \\
\hline C12 & 2.21 & 2.23 & 2.19 & 2.14 & 2.14 & 2.04 \\
\hline $\mathrm{C} 13$ & 1.51 & 1.81 & 1.84 & 1.67 & 1.71 & 1.64 \\
\hline C14 & 1.28 & 1.82 & 1.68 & 1.60 & 1.60 & 1.58 \\
\hline C15 & 1.04 & 2.07 & 1.64 & 1.58 & 1.58 & 1.48 \\
\hline alpha chain growth probability & 0.70 & 0.75 & 0.74 & 0.74 & 0.74 & 0.73 \\
\hline \multicolumn{7}{|c|}{ C1 - C50 estimated total product distribution, weight \% } \\
\hline $\mathrm{C} 1$ & 18.7 & 18.5 & 20.3 & 20.9 & 22.2 & 21.8 \\
\hline $\mathrm{C} 2-\mathrm{C} 4$ & 24.2 & 21.2 & 22.1 & 22.4 & 20.2 & 22.6 \\
\hline $\mathrm{C} 5-\mathrm{C} 12$ & 50.1 & 46.5 & 46.5 & 46.0 & 46.9 & 45.6 \\
\hline $\mathrm{C} 13-\mathrm{C} 50$ & 7.0 & 13.9 & 11.1 & 10.7 & 10.7 & 10.0 \\
\hline CO conversion, \% & 3.8 & 3.6 & 3.2 & 3.0 & 2.7 & 2.7 \\
\hline rate, $\mathrm{g} \mathrm{CH} 2 / \mathrm{g} \mathrm{cat} / \mathrm{hr}$ & 0.23 & 0.22 & 0.19 & 0.18 & 0.17 & 0.17 \\
\hline $\mathrm{CO} 2$ formation, $\%$ & 0.1 & 0.1 & 0.1 & 0.1 & 0.1 & 0.1 \\
\hline
\end{tabular}




\section{Performance of Co.045}

Dates: 12/03/94 - 12/04/94 Run \#1

\begin{tabular}{lcc} 
flow rate $=90.0 \mathrm{cc} / \mathrm{min}$, loading $=0.2 \mathrm{~g}$, WHSV $=13.9$ & $1 / \mathrm{hr}, \mathrm{H} 2 / \mathrm{CO}$ ratio in feed $=2$ \\
\hline & 18.5 & 21.5 \\
time on stream, hr & 220 & 220 \\
reaction temperature, ${ }^{\circ} \mathrm{C}$ & 1.0 & 1.0 \\
pressure, atm & 90.0 & 90.0 \\
flow, cc/min & & \\
\hline & & \\
C1 - C15 product distribution, weight \% & & \\
& & \\
C1 & 23.70 & 24.15 \\
& & \\
C2 & 5.16 & 5.26 \\
C3 & 9.61 & 9.73 \\
C4 & 9.73 & 9.81 \\
& & \\
C5 & 10.52 & 10.40 \\
C6 & 8.74 & 8.78 \\
C7 & 7.98 & 8.08 \\
C8 & 6.14 & 6.18 \\
C9 & 4.69 & 4.64 \\
C10 & 3.72 & 3.66 \\
C11 & 2.79 & 2.70 \\
C12 & 2.57 & 2.03 \\
C13 & 1.65 & 1.56 \\
C14 & 1.51 & 1.50 \\
C15 & 1.50 & 1.52 \\
& & \\
alpha chain growth probability & 0.72 & 0.73 \\
\end{tabular}

C1 - C50 estimated total product distribution, weight \%

$\begin{array}{lcc}\text { C1 } 122.0 & 22.2 \\ \text { C2 - C4 } & 22.7 & 22.8 \\ \text { C5 - C12 } & 45.4 & 44.8 \\ \text { C13 - C50 } & 9.9 & 10.2\end{array}$

$\begin{array}{lll}\mathrm{CO} \text { conversion, } \% & 2.7 & 2.6 \\ \mathrm{rate}, \mathrm{g} \mathrm{CH} 2 / \mathrm{g} \mathrm{cat} / \mathrm{hr} & 0.16 & 0.16 \\ \mathrm{CO} 2 \text { formation, \% } & 0.1 & 0.1\end{array}$


Time-on-Stream Plot for Co.045 - Run \#1

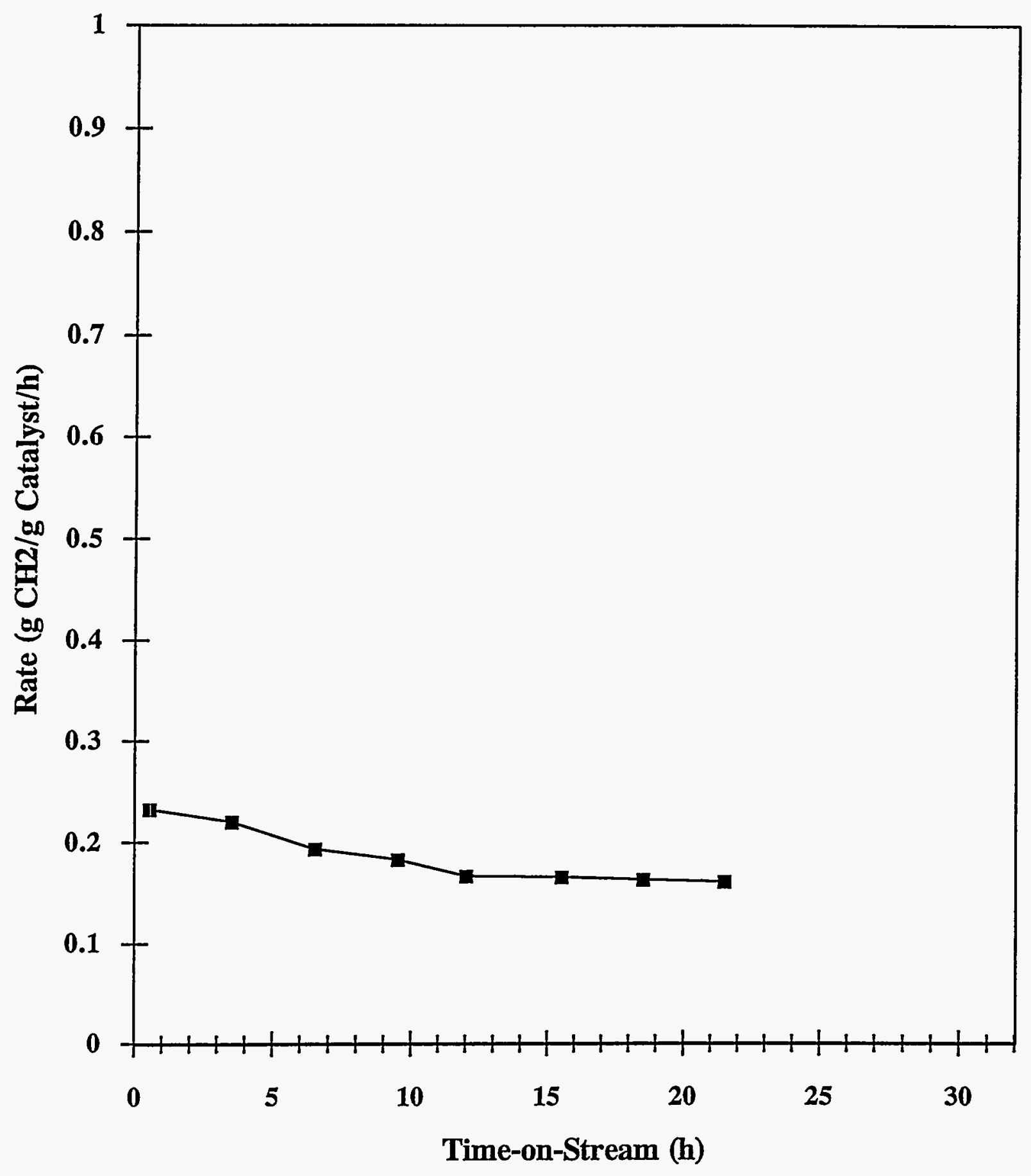


Schulz-Flory Plot for Co.045 - Run \#1

Time on Stream (hrs)

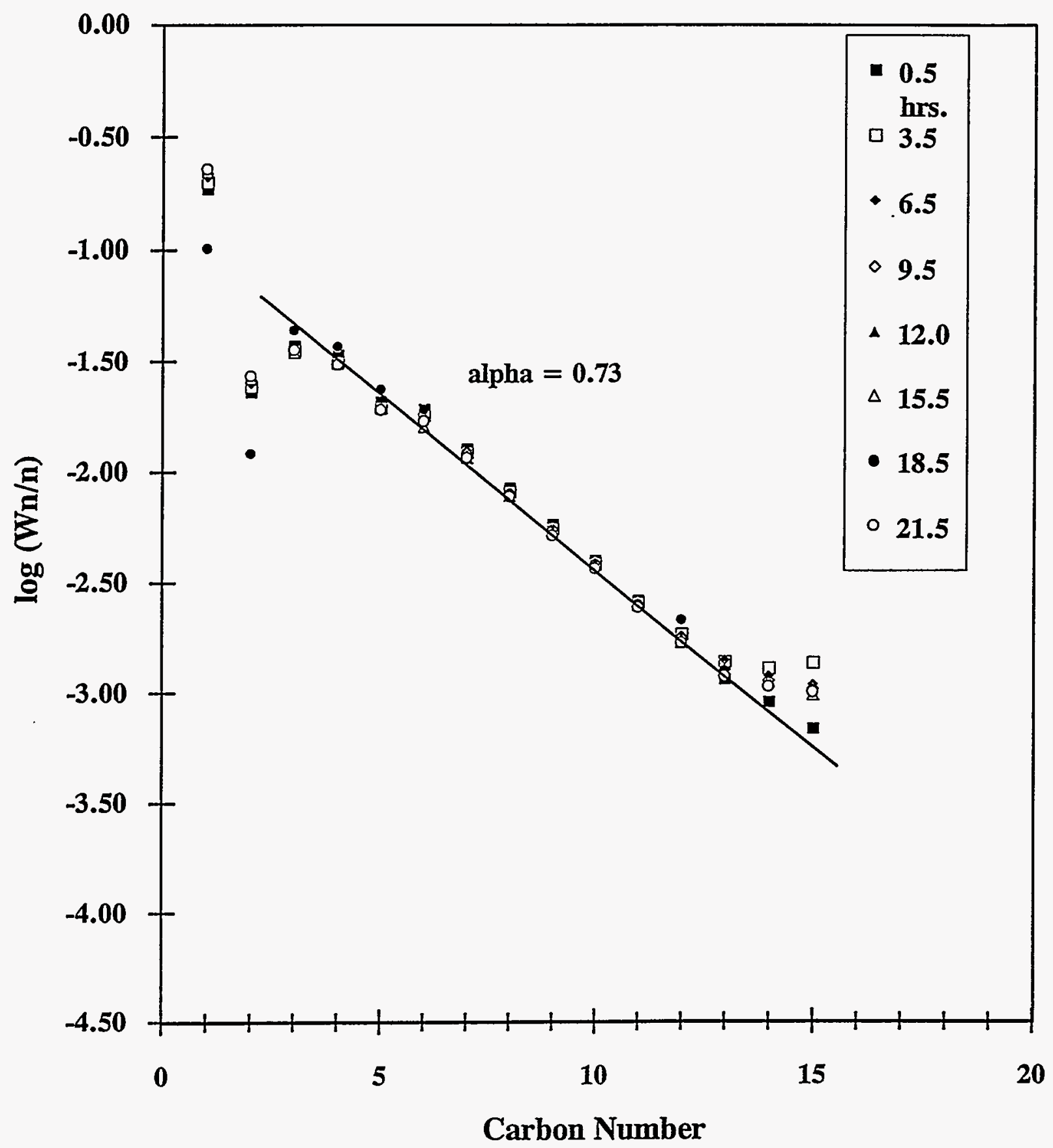




\section{Co.053 - Run \#4}

\begin{tabular}{||c||cc||c|c||c|}
\hline \hline Co wt\% & NM & wt \% & \multicolumn{2}{|c||}{ Promotor wt\% } & Support \\
\hline 20 & Ru & 0.50 & & & Al2O3 \\
\hline
\end{tabular}

\section{SUMMARY REACTION DATA*}

Reaction Conditions:

$$
\begin{array}{ll}
\mathrm{P}=1.0 \mathrm{~atm} & \mathrm{CO} 2(\mathrm{~g} / \mathrm{g} \mathrm{cat} / \mathrm{hr})=0.030 \\
\mathrm{~T}=220^{\circ} \mathrm{C} & \mathrm{CO} 2(\% \text { of } \mathrm{CO})=0.1 \\
\mathrm{H} 2 / \mathrm{CO}=2 & \mathrm{O} / \mathrm{P}=1.41 \\
\text { weight of catalyst }=0.160 \mathrm{~g} & \\
\text { WHSV }=16.071 / \mathrm{hr} & \\
\text { time on stream }=18.5 \mathrm{hrs} &
\end{array}
$$

\begin{tabular}{||l|l|}
\hline CO conversion (\%) & 6.1 \\
\hline rate (g CH2/g cat/hr) & 0.43 \\
\hline alpha & 0.65 \\
\hline C1 (wt\%) & 28.3 \\
\hline C2 - C4 (wt\%) & 29.0 \\
\hline C5 - C12 (wt\%) & 39.6 \\
\hline C13 + (wt\%) & 3.1 \\
\hline
\end{tabular}

* Catalyst reduced without calcination 


\section{Performance of Co.053}

Dates: 10/12/94 - 10/13/94 Run \#4

flow rate $=90.0 \mathrm{cc} / \mathrm{min}$, loading $=0.2 \mathrm{~g}, \mathrm{WHSV}=16.1 \mathrm{1} / \mathrm{hr}, \mathrm{H} 2 / \mathrm{CO}$ ratio in feed $=2$

\begin{tabular}{|c|c|c|c|c|c|c|}
\hline time on stream, $\mathrm{hr}$ & 3.5 & 9.5 & 12.5 & 15.5 & 18.5 & 24.5 \\
\hline reaction temperature, ${ }^{\circ} \mathrm{C}$ & 220 & 220 & 220 & 220 & 220 & 220 \\
\hline pressure, atm & 1.0 & 1.0 & 1.0 & 1.0 & 1.0 & 1.0 \\
\hline flow, cc/min & 90.0 & 90.0 & 90.0 & 90.0 & 90.0 & 90.0 \\
\hline \multicolumn{7}{|c|}{ C1 - C15 product distribution, weight \% } \\
\hline $\mathrm{C} 1$ & 29.00 & 29.18 & 27.03 & 28.75 & 28.98 & 29.41 \\
\hline $\mathrm{C} 2$ & 5.16 & 5.17 & 12.96 & 5.08 & 5.11 & 5.21 \\
\hline C3 & 12.81 & 12.69 & 11.64 & 12.44 & 12.50 & 12.74 \\
\hline $\mathrm{C} 4$ & 12.41 & 12.29 & 11.24 & 12.13 & 12.14 & 12.27 \\
\hline $\mathrm{C5}$ & 11.08 & 10.94 & 10.05 & 11.07 & 11.00 & 10.98 \\
\hline C6 & 7.66 & 7.24 & 6.80 & 7.85 & 7.85 & 7.27 \\
\hline C7 & 7.57 & 7.60 & 6.80 & 7.63 & 7.65 & 7.54 \\
\hline C8 & 4.95 & 5.08 & 4.62 & 5.08 & 5.09 & 5.01 \\
\hline $\mathrm{CP}$ & 3.28 & 3.37 & 3.10 & 3.42 & 3.36 & 3.42 \\
\hline $\mathrm{C} 10$ & 2.13 & 2.28 & 2.06 & 2.25 & 2.25 & 2.26 \\
\hline $\mathrm{C} 11$ & 1.41 & 1.51 & 1.34 & 1.48 & 1.55 & 1.51 \\
\hline $\mathrm{C} 12$ & 0.90 & 1.01 & 0.84 & 1.09 & 0.85 & 0.98 \\
\hline $\mathrm{C} 13$ & 0.71 & 0.67 & 0.61 & 0.68 & 0.65 & 0.64 \\
\hline C14 & 0.58 & 0.56 & 0.52 & 0.58 & 0.56 & 0.52 \\
\hline C15 & 0.34 & 0.41 & 0.40 & 0.47 & 0.47 & 0.24 \\
\hline alpha chain growth probability & 0.63 & 0.64 & 0.64 & 0.65 & 0.65 & 0.61 \\
\hline \multicolumn{7}{|c|}{ C1 - C50 estimated total product distribution, weight \% } \\
\hline $\mathrm{C} 1$ & 28.7 & 28.7 & 26.5 & 28.1 & 28.3 & 29.4 \\
\hline $\mathrm{C} 2-\mathrm{C} 4$ & 30.1 & 29.7 & 35.1 & 29.0 & 29.0 & 30.3 \\
\hline C5 - C12 & 39.0 & 38.9 & 35.7 & 39.8 & 39.6 & 38.7 \\
\hline $\mathrm{C} 13-\mathrm{C} 50$ & 2.2 & 2.7 & 2.6 & 3.1 & 3.1 & 1.6 \\
\hline CO conversion, $\%$ & 6.8 & 6.2 & 6.8 & 6.1 & 6.1 & 5.2 \\
\hline rate, g CH2/g cat $/ \mathrm{hr}$ & 0.48 & 0.43 & 0.48 & 0.43 & 0.43 & 0.37 \\
\hline $\mathrm{CO} 2$ formation, $\%$ & 0.2 & 0.2 & 0.2 & 0.1 & 0.1 & 0.2 \\
\hline
\end{tabular}




\section{Performance of Co.053}

\section{Dates: 10/12/94 - 10/13/94 Run \#4}

flow rate $=90.0 \mathrm{cc} / \mathrm{min}$, loading $=0.2 \mathrm{~g}, \mathrm{WHSV}=16.1 \mathrm{1} / \mathrm{hr}, \mathrm{H} 2 / \mathrm{CO}$ ratio in feed $=2$

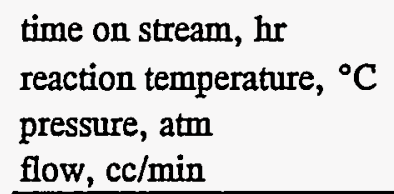


Time-on-Stream Plot for Co.053 - Run \#4

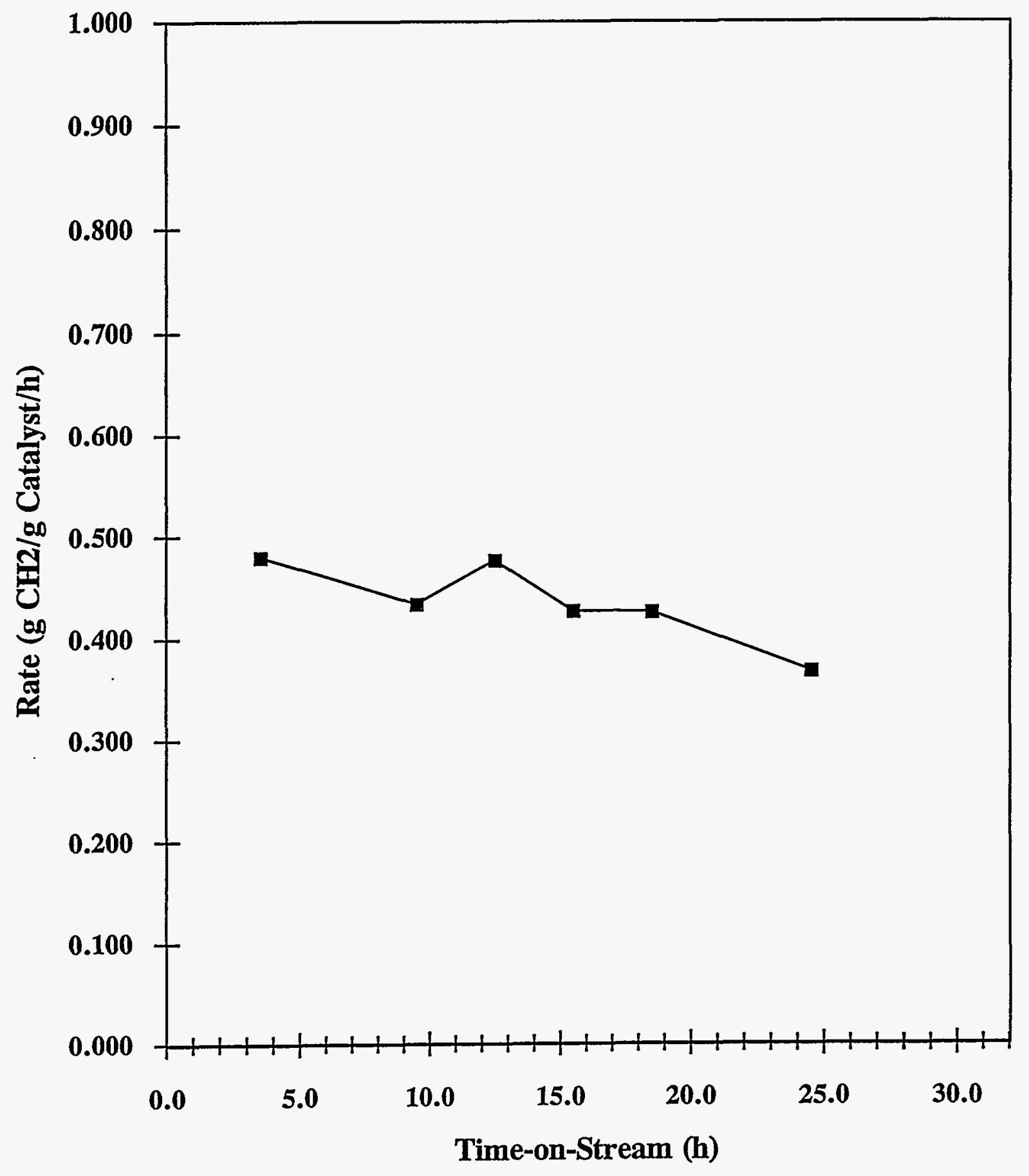


Schulz-Flory Plot for Co.053 - Run \#4

Time on Stream (hrs)

hrs.

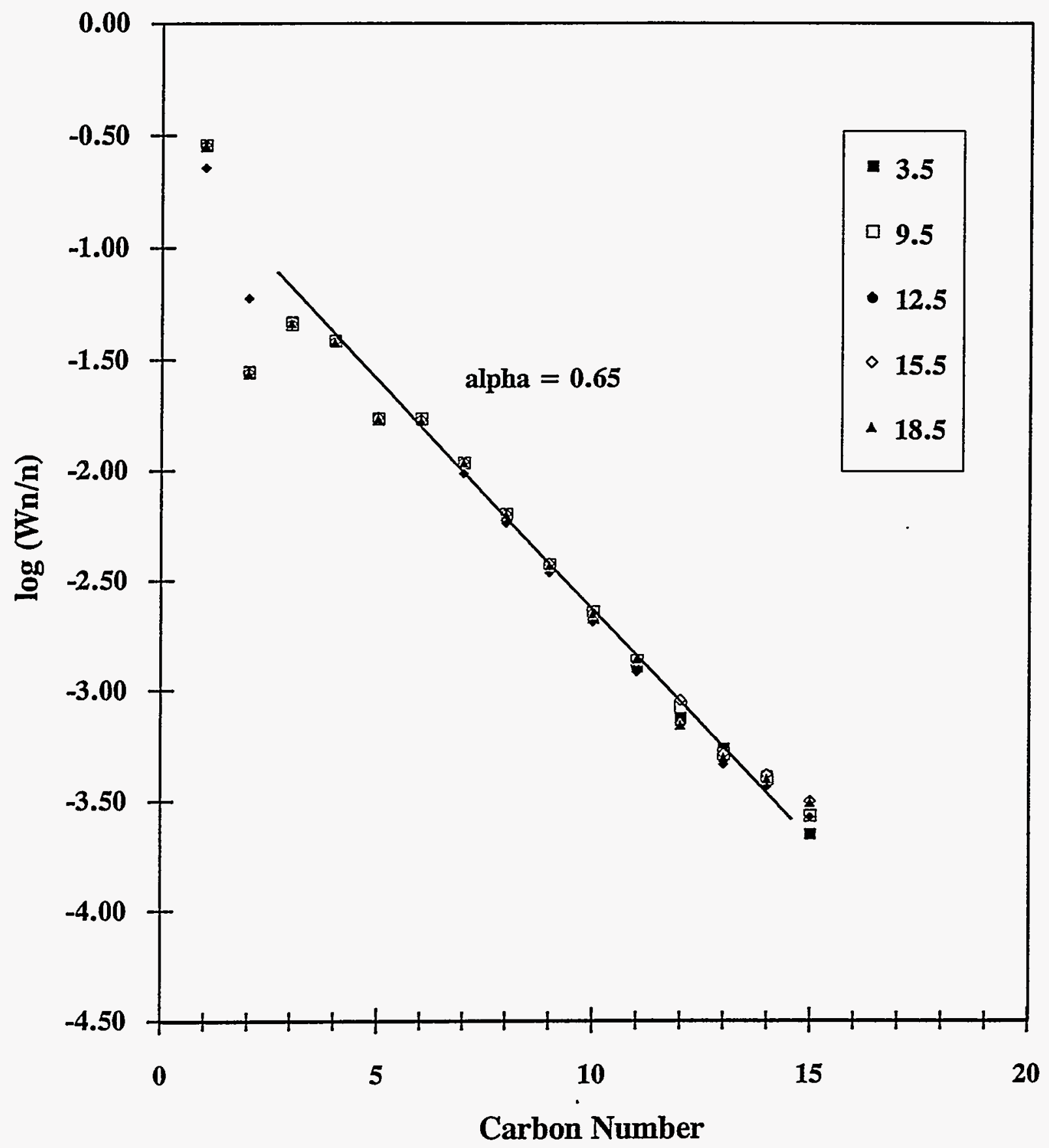




\section{Co.053 - Run \#5}

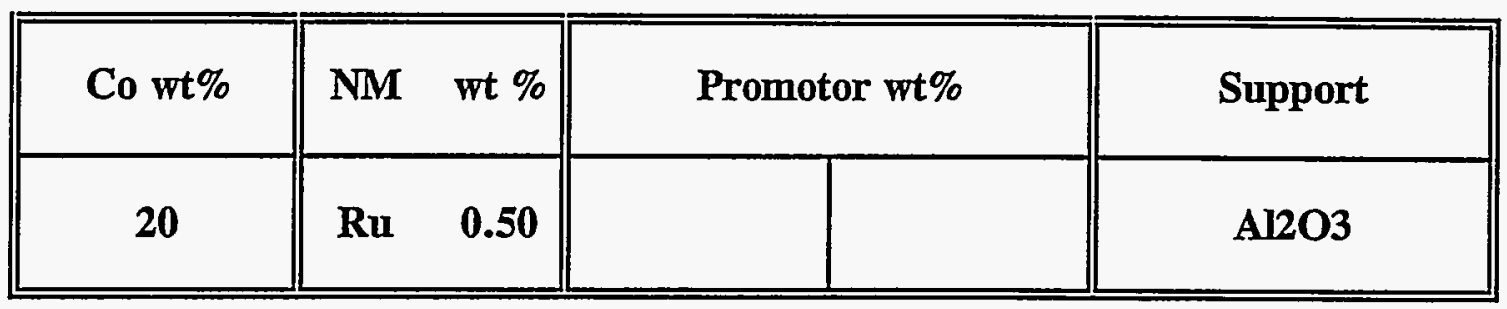

\section{SUMMARY REACTION DATA*}

Reaction Conditions:

$$
\begin{array}{ll}
\mathrm{P}=1.0 \mathrm{~atm} & \mathrm{CO} 2(\mathrm{~g} / \mathrm{g} \mathrm{cat} / \mathrm{hr})=0.034 \\
\mathrm{~T}=220^{\circ} \mathrm{C} & \mathrm{CO} 2(\% \text { of } \mathrm{CO})=0.2 \\
\mathrm{H} 2 / \mathrm{CO}=2 & \mathrm{O} / \mathrm{P}=1.49 \\
\text { weight of catalyst }=0.175 \mathrm{~g} & \\
\text { WHSV }=14.691 / \mathrm{hr} & \\
\text { time on stream }=27.5 \mathrm{hrs} &
\end{array}
$$

\begin{tabular}{|l|c|}
\hline \hline CO conversion (\%) & 5.6 \\
\hline rate (g CH2/g cat/hr) & 0.36 \\
\hline alpha & 0.64 \\
\hline $\mathrm{C} 1$ (wt\%) & 27.9 \\
\hline $\mathrm{C} 2-\mathrm{C} 4(\mathrm{wt} \%)$ & 29.5 \\
\hline $\mathrm{C} 5-\mathrm{C} 12(\mathrm{wt} \%)$ & 40.0 \\
\hline $\mathrm{C} 13+(\mathrm{wt} \%)$ & 2.6 \\
\hline
\end{tabular}

* Catalyst is reduced, calcined and rereduced 


\section{Performance of Co.053}

Dates: 10/17/94 - 10/18/94 Run \#5

flow rate $=90.0 \mathrm{cc} / \mathrm{min}$, loading $=0.2 \mathrm{~g}, \mathrm{WHSV}=14.71 / \mathrm{hr}, \mathrm{H} 2 / \mathrm{CO}$ ratio in feed $=2$

\begin{tabular}{|c|c|c|c|c|c|c|}
\hline time on stream, hr & 0.5 & 9.5 & 12.5 & 15.5 & 24.5 & 27.5 \\
\hline reaction temperature, ${ }^{\circ} \mathrm{C}$ & 220 & 220 & 220 & 220 & 220 & 220 \\
\hline pressure, atm & 1.0 & 1.0 & 1.0 & 1.0 & 1.0 & 1.0 \\
\hline flow, cc/min & 90.0 & 90.0 & 90.0 & 90.0 & 90.0 & 90.0 \\
\hline \multicolumn{7}{|c|}{$\mathrm{Cl}-\mathrm{C} 15$ product distribution, weight \% } \\
\hline $\mathrm{Cl}$ & 26.42 & 28.47 & 28.57 & 28.76 & 28.17 & 28.48 \\
\hline $\mathrm{C} 2$ & 4.77 & 5.07 & 5.07 & 5.07 & 4.96 & 5.01 \\
\hline C3 & 12.73 & 13.04 & 12.99 & 12.91 & 12.66 & 12.70 \\
\hline C4 & 12.71 & 12.70 & 12.65 & 12.52 & 12.36 & 12.34 \\
\hline C5 & 11.66 & 11.30 & 11.26 & 11.22 & 11.38 & 11.24 \\
\hline C6 & 8.43 & 7.52 & 7.41 & 7.54 & 8.21 & 8.05 \\
\hline C7 & 8.17 & 7.88 & 7.84 & 7.75 & 7.93 & 7.83 \\
\hline $\mathrm{C} 8$ & 5.27 & 5.06 & 5.17 & 5.09 & 5.16 & 5.06 \\
\hline C9 & 3.47 & 3.26 & 3.20 & 3.33 & 3.32 & 3.33 \\
\hline $\mathrm{C} 10$ & 2.10 & 2.06 & 2.09 & 2.06 & 2.17 & 2.19 \\
\hline C11 & 1.55 & 1.35 & 1.34 & 1.33 & 1.26 & 1.30 \\
\hline $\mathrm{C} 12$ & 1.08 & 0.85 & 0.84 & 0.82 & 0.82 & 0.87 \\
\hline $\mathrm{C} 13$ & 0.87 & 0.68 & 0.68 & 0.66 & 0.66 & 0.64 \\
\hline C14 & 0.59 & 0.51 & 0.55 & 0.55 & 0.56 & 0.56 \\
\hline C15 & 0.19 & 0.24 & 0.34 & 0.39 & 0.37 & 0.40 \\
\hline alpha chain growth probability & 0.59 & 0.61 & 0.62 & 0.63 & 0.63 & 0.64 \\
\hline \multicolumn{7}{|c|}{ C1 - C50 estimated total product distribution, weight \% } \\
\hline $\mathrm{C} 1$ & 26.7 & 28.4 & 28.2 & 28.2 & 27.7 & 27.9 \\
\hline $\mathrm{C} 2-\mathrm{C} 4$ & 30.5 & 30.8 & 30.3 & 29.9 & 29.5 & 29.5 \\
\hline $\mathrm{C} 5-\mathrm{C} 12$ & 41.4 & 39.2 & 39.2 & 39.3 & 40.4 & 40.0 \\
\hline $\mathrm{C} 13-\mathrm{C} 50$ & 1.3 & 1.6 & 2.2 & 2.6 & 2.5 & 2.6 \\
\hline CO conversion, \% & 6.7 & 5.7 & 5.6 & 5.8 & 5.5 & 5.6 \\
\hline rate, g CH $2 / g$ cat $/ \mathrm{hr}$ & 0.43 & 0.37 & 0.36 & 0.37 & 0.36 & 0.36 \\
\hline $\mathrm{CO} 2$ formation, $\%$ & 0.2 & 0.2 & 0.2 & 0.2 & 0.1 & 0.2 \\
\hline
\end{tabular}


Time-on-Stream Plot for Co.053 - Run \#5

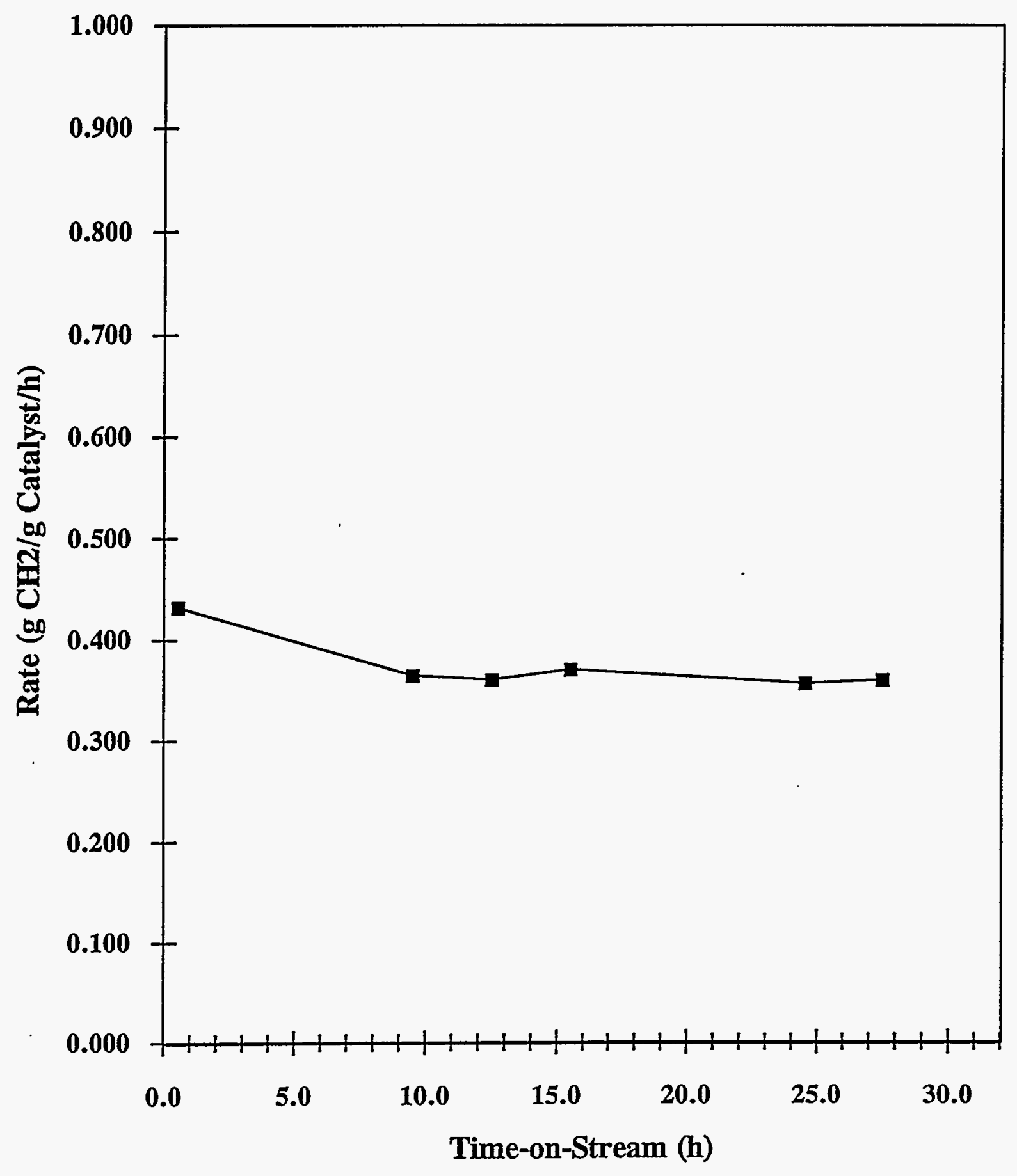


Schulz-Flory Plot for Co.053 - Run \#5

Time on Stream (hrs)

brs.

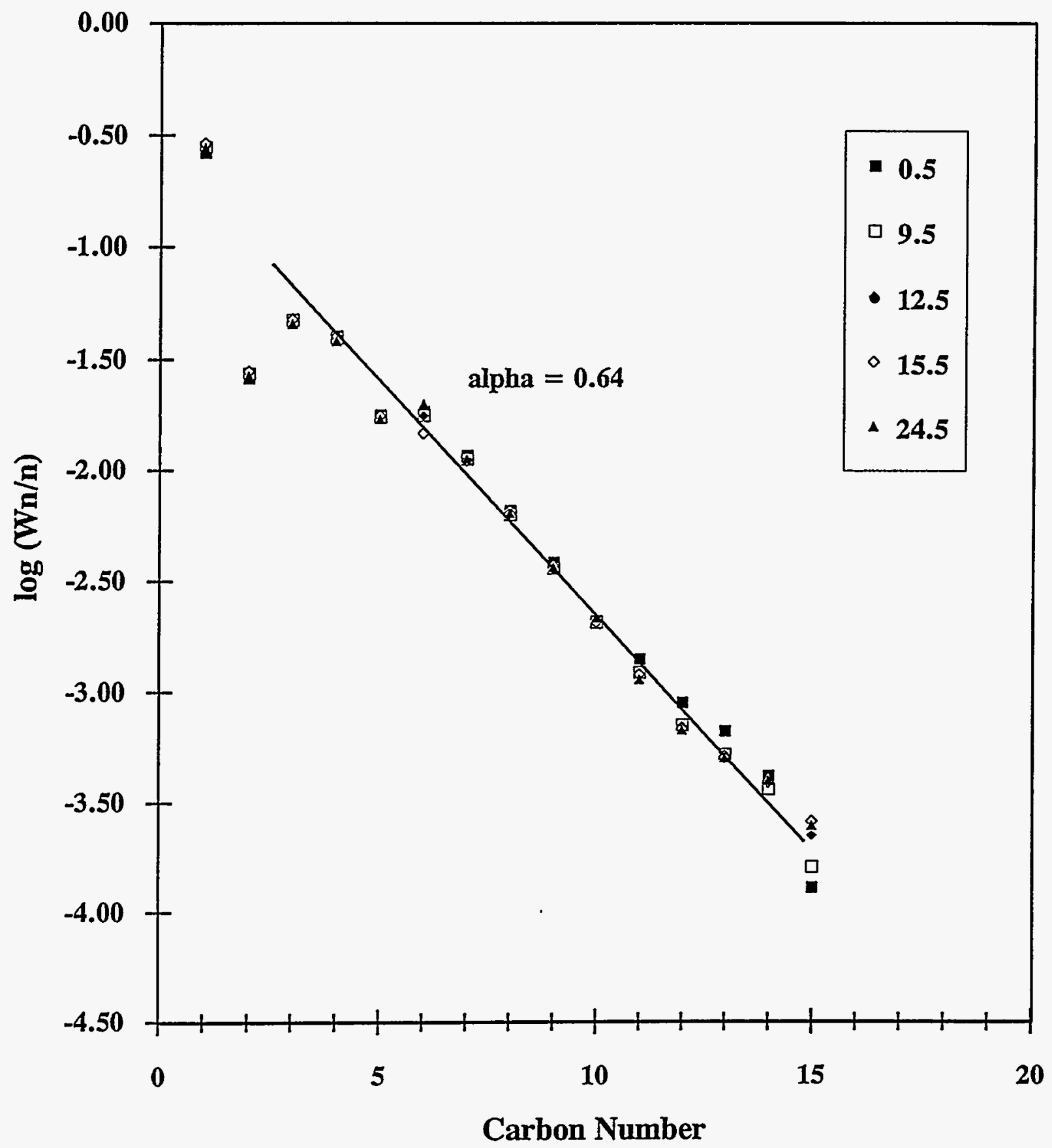




\section{Co.053 - Run \#6}

\begin{tabular}{|c||cc||c|c|c|}
\hline \hline Co wt\% & NM & wt \% & \multicolumn{2}{|c||}{ Promotor wt\% } & Support \\
\hline 20 & Ru & 0.50 & & & Al2O3 \\
\hline
\end{tabular}

\section{SUMMARY REACTION DATA}

Reaction Conditions:

$$
\begin{array}{ll}
\mathrm{P}=1.0 \mathrm{~atm} & \mathrm{CO} 2(\mathrm{~g} / \mathrm{g} \mathrm{cat} / \mathrm{hr})=0.030 \\
\mathrm{~T}=220^{\circ} \mathrm{C} & \mathrm{CO} 2(\% \text { of CO})=0.1 \\
\mathrm{H} 2 / \mathrm{CO}=2 & \mathrm{O} / \mathrm{P}=1.25 \\
\text { weight of catalyst }=0.191 \mathrm{~g} & \\
\text { WHSV }=13.501 / \mathrm{hr} & \\
\text { time on stream }=24.5 \mathrm{hrs} &
\end{array}
$$

\begin{tabular}{||l|c|}
\hline \hline CO conversion (\%) & 8.2 \\
\hline rate (g CH2/g cat/hr) & 0.49 \\
\hline alpha & 0.64 \\
\hline C1 (wt\%) & 28.9 \\
\hline C2 - C4 (wt\%) & 28.1 \\
\hline C5 - C12 (wt\%) & 40.0 \\
\hline C13 + (wt\%) & 3.0 \\
\hline
\end{tabular}




\section{Performance of Co.053}

\section{Dates: 11/03/94 - 11/04/94 Run \#6}

flow rate $=90.0 \mathrm{cc} / \mathrm{min}$, loading $=0.2 \mathrm{~g}, \mathrm{WHSV}=13.51 / \mathrm{br}, \mathrm{H} 2 / \mathrm{CO}$ ratio in feed $=2$

\begin{tabular}{|c|c|c|c|c|c|c|}
\hline time on stream, hr & 0.5 & 3.5 & 6.5 & 9.5 & 12.5 & 15.5 \\
\hline reaction temperature, ${ }^{\circ} \mathrm{C}$ & 220 & 220 & 220 & 220 & 220 & 220 \\
\hline pressure, atm & 1.0 & 1.0 & 1.0 & 1.0 & 1.0 & 1.0 \\
\hline flow, cc/min & 90.0 & 90.0 & 90.0 & 90.0 & 90.0 & 90.0 \\
\hline \multicolumn{7}{|c|}{ C1 - C15 product distribution, weight \% } \\
\hline $\mathrm{C} 1$ & 31.34 & 31.17 & 32.00 & 31.40 & 31.63 & 31.31 \\
\hline $\mathrm{C} 2$ & 5.30 & 5.20 & 5.28 & 5.17 & 5.17 & 5.13 \\
\hline C3 & 12.76 & 12.61 & 12.70 & 12.58 & 12.55 & 12.57 \\
\hline $\mathrm{C4}$ & 12.68 & 12.66 & 12.60 & 12.43 & 12.31 & 12.39 \\
\hline C5 & 11.34 & 11.24 & 11.14 & 11.19 & 11.07 & 11.15 \\
\hline C6 & 8.30 & 8.31 & 7.63 & 8.60 & 8.37 & 8.55 \\
\hline $\mathrm{C7}$ & 6.38 & 6.40 & 6.49 & 6.51 & 6.51 & 6.51 \\
\hline $\mathrm{C} 8$ & 4.14 & 4.17 & 4.26 & 4.28 & 4.34 & 4.41 \\
\hline C9 & 2.70 & 2.69 & 2.77 & 2.82 & 2.86 & 2.86 \\
\hline C10 & 1.70 & 1.72 & 1.79 & 1.75 & 1.80 & 1.80 \\
\hline C11 & 1.20 & 1.23 & 1.22 & 1.20 & 1.23 & 1.27 \\
\hline $\mathrm{C} 12$ & 0.74 & 0.72 & 0.76 & 0.78 & 0.81 & 0.77 \\
\hline $\mathrm{C} 13$ & 0.62 & 0.60 & 0.51 & 0.51 & 0.52 & 0.48 \\
\hline $\mathrm{Cl} 4$ & 0.45 & 1.16 & 0.43 & 0.42 & 0.44 & 0.42 \\
\hline $\mathrm{C} 15$ & 0.35 & 0.11 & 0.42 & 0.37 & 0.38 & 0.37 \\
\hline alpha chain growth probability & 0.63 & 0.56 & 0.64 & 0.63 & 0.63 & 0.63 \\
\hline \multicolumn{7}{|c|}{ C1 - C50 estimated total product distribution, weight \% } \\
\hline $\mathrm{C} 1$ & 30.8 & 31.8 & 31.2 & 30.7 & 31.0 & 30.7 \\
\hline $\mathrm{C} 2-\mathrm{C} 4$ & 30.2 & 31.0 & 29.8 & 29.6 & 29.4 & 29.5 \\
\hline $\mathrm{C} 5-\mathrm{C} 12$ & 36.7 & 36.4 & 36.3 & 37.3 & 37.1 & 37.4 \\
\hline $\mathrm{C} 13-\mathrm{C} 50$ & 2.3 & 0.7 & 2.8 & 2.4 & 2.5 & 2.4 \\
\hline CO conversion, \% & 10.4 & 9.1 & 9.2 & 8.7 & 8.8 & 8.4 \\
\hline rate, g CH $2 / g$ cat $/ \mathrm{hr}$ & 0.62 & 0.54 & 0.54 & 0.51 & 0.52 & 0.49 \\
\hline $\mathrm{CO} 2$ formation, $\%$ & 0.3 & 0.2 & 0.2 & 0.2 & 0.1 & 0.2 \\
\hline
\end{tabular}




\section{Performance of Co.053}

Dates: 11/03/94 - 11/04/94 Run \#6

flow rate $=90.0 \mathrm{cc} / \mathrm{min}$, loading $=0.2 \mathrm{~g}, \mathrm{WHSV}=13.51 / \mathrm{hr}, \mathrm{H} 2 / \mathrm{CO}$ ratio in feed $=2$

\begin{tabular}{|c|c|c|c|}
\hline time on stream, $\mathrm{hr}$ & 18.5 & 21.5 & 24.5 \\
\hline reaction temperature, ${ }^{\circ} \mathrm{C}$ & 220 & 220 & 220 \\
\hline pressure, atm & 1.0 & 1.0 & 1.0 \\
\hline flow, cc/min & 90.0 & 90.0 & 90.0 \\
\hline \multicolumn{4}{|c|}{ C1 - C15 product distribution, weight \% } \\
\hline $\mathrm{C} 1$ & 31.24 & 30.77 & 29.63 \\
\hline $\mathrm{C} 2$ & 5.11 & 5.05 & 4.86 \\
\hline C3 & 12.52 & 12.51 & 12.09 \\
\hline $\mathrm{C} 4$ & 12.30 & 10.77 & 11.84 \\
\hline $\mathrm{C5}$ & 11.17 & 11.22 & 10.90 \\
\hline $\mathrm{C} 6$ & 8.55 & 6.67 & 8.49 \\
\hline $\mathrm{C7}$ & 6.63 & 7.90 & 7.62 \\
\hline $\mathrm{C} 8$ & 4.41 & 5.26 & 5.11 \\
\hline C9 & 2.90 & 3.45 & 3.37 \\
\hline $\mathrm{C} 10$ & 1.83 & 2.24 & 2.16 \\
\hline $\mathrm{C} 11$ & 1.22 & 1.52 & 1.46 \\
\hline $\mathrm{C} 12$ & 0.79 & 1.02 & 0.90 \\
\hline $\mathrm{C} 13$ & 0.51 & 0.61 & 0.59 \\
\hline $\mathrm{C} 14$ & 0.44 & 0.54 & 0.51 \\
\hline $\mathrm{C} 15$ & 0.37 & 0.46 & 0.45 \\
\hline alpha chain growth probability & 0.63 & 0.65 & 0.64 \\
\hline
\end{tabular}

C1 - C50 estimated total product distribution, weight \%

C1

C2 - C4

C5 - C12

C13 - C50 $\begin{array}{lll}30.6 & 30.1 & 28.9\end{array}$

$29.3 \quad 27.7 \quad 28.1$

$\begin{array}{lll}37.6 & 39.2 & 40.0\end{array}$

$\begin{array}{lll}2.4 & 3.0 & 3.0\end{array}$
CO conversion, $\%$ rate, $\mathrm{g} \mathrm{CH} 2 / \mathrm{g} \mathrm{cat} / \mathrm{hr}$

$\mathrm{CO} 2$ formation, \% $\begin{array}{lll}8.3 & 8.1 & 8.2\end{array}$

$\begin{array}{lll}0.49 & 0.48 & 0.49\end{array}$

$\begin{array}{lll}0.2 & 0.1 & 0.1\end{array}$ 
Time-on-Stream Plot for Co.053 - Run \#6

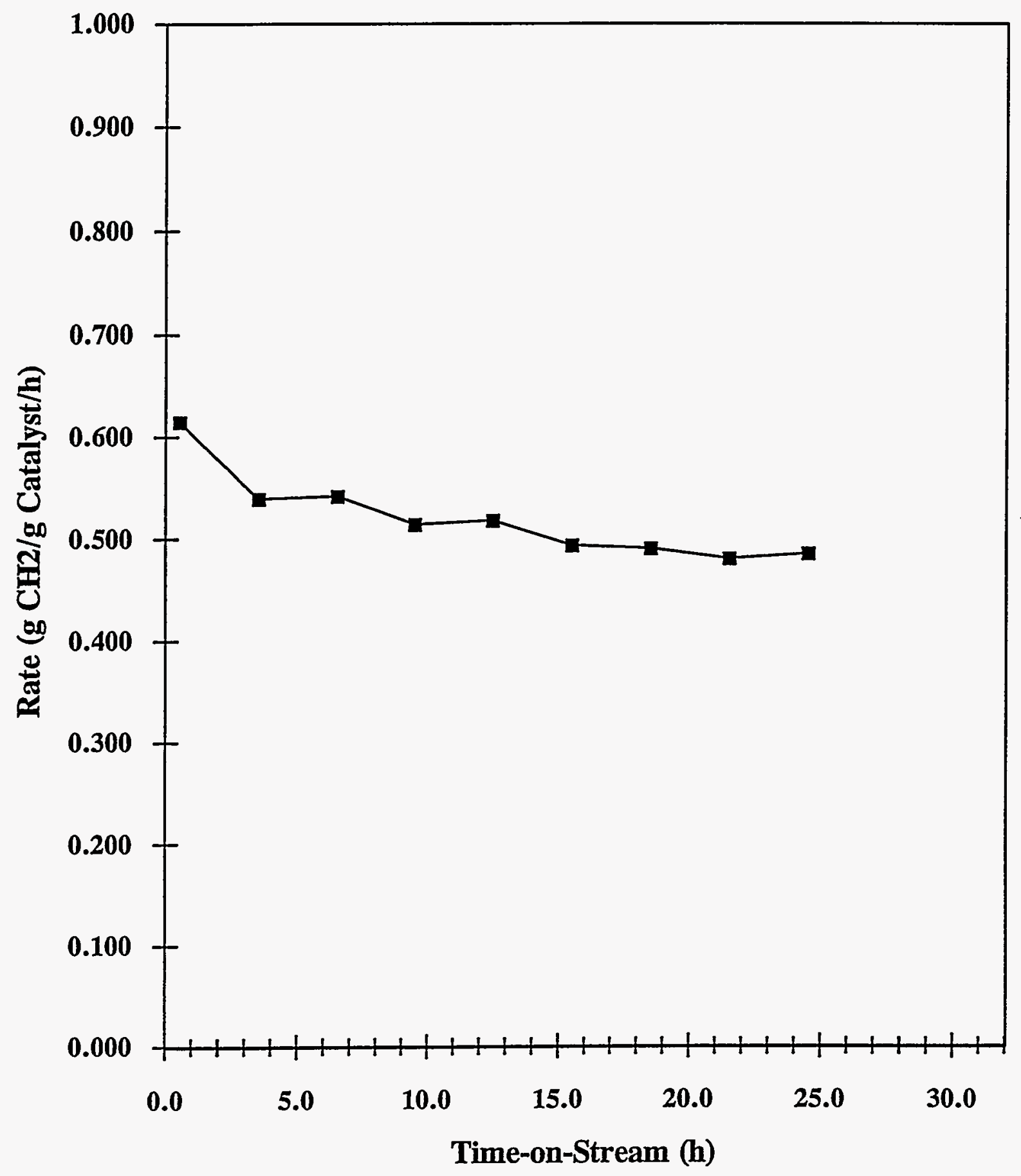


Schulz-Flory Plot for Co.053 - Run \#6

Time on Stream (hrs)

hrs.

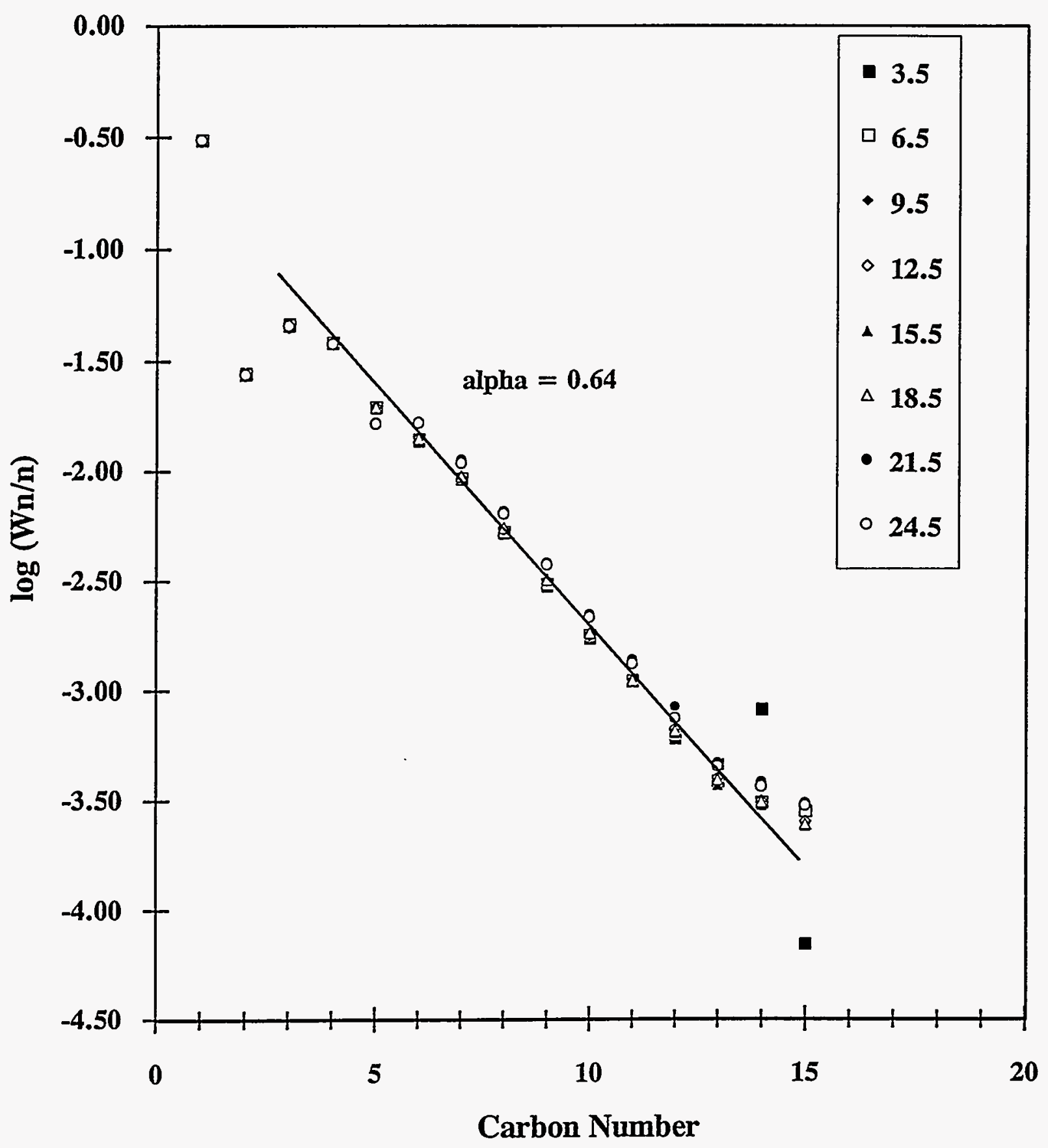




\section{Co.055 - Run \#4}

\begin{tabular}{|c|c|c|c|c|}
\hline Co wt\% & NM & wt \% & Promotor wt\% & Support \\
\hline 20 & $\mathbf{R e}$ & 1.00 & $\mathrm{La2O3} 1.00$ & $\mathrm{Al} 2 \mathrm{O} 3$ \\
\hline
\end{tabular}

\section{SUMMARY REACTION DATA*}

Reaction Conditions:

$$
\begin{array}{ll}
\mathrm{P}=1.0 \mathrm{~atm} & \mathrm{CO} 2(\mathrm{~g} / \mathrm{g} \mathrm{cat} / \mathrm{hr})=0.047 \\
\mathrm{~T}=220^{\circ} \mathrm{C} & \mathrm{CO} 2(\% \text { of } \mathrm{CO})=0.2 \\
\mathrm{H} 2 / \mathrm{CO}=2 & \mathrm{O} / \mathrm{P}=1.94 \\
\text { weight of catalyst }=0.174 \mathrm{~g} & \\
\text { WHSV }=14.801 / \mathrm{hr} & \\
\text { time on stream }=21.5 \mathrm{hrs} &
\end{array}
$$

\begin{tabular}{||l|c|}
\hline CO conversion (\%) & 5.9 \\
\hline rate (g CH2/g cat/hr) & 0.38 \\
\hline alpha & 0.60 \\
\hline $\mathrm{C} 1(\mathrm{wt} \%)$ & 28.3 \\
\hline $\mathrm{C} 2-\mathrm{C} 4(\mathrm{wt} \%)$ & 29.2 \\
\hline $\mathrm{C} 5-\mathrm{C} 12(\mathrm{wt} \%)$ & 41.0 \\
\hline $\mathrm{C} 13+(\mathrm{wt} \%)$ & 1.4 \\
\hline
\end{tabular}

* Catalyst calcined without calcination 


\title{
Performance of Co.055
}

\author{
Dates: 10/20/94 - 10/21/94 Run \#4
}

flow rate $=90.0 \mathrm{cc} / \mathrm{min}$, loading $=0.2 \mathrm{~g}, \mathrm{WHSV}=14.81 / \mathrm{hr}, \mathrm{H} 2 / \mathrm{CO}$ ratio in feed $=2$

\begin{tabular}{|c|c|c|c|c|c|}
\hline time on stream, hr & 3.5 & 6.5 & 9.5 & 12.5 & 21.5 \\
\hline reaction temperature, ${ }^{\circ} \mathrm{C}$ & 220 & 220 & 220 & 220 & 220 \\
\hline pressure, atm & 1.0 & 1.0 & 1.0 & 1.0 & 1.0 \\
\hline flow, cc/min & 90.0 & 90.0 & 90.0 & 90.0 & 90.0 \\
\hline \multicolumn{6}{|c|}{$\mathrm{C} 1-\mathrm{C} 15$ product distribution, weight $\%$} \\
\hline $\mathrm{Cl}$ & 28.31 & 27.51 & 27.23 & 28.09 & 27.97 \\
\hline $\mathrm{C} 2$ & 4.94 & 4.80 & 4.71 & 4.85 & 4.84 \\
\hline $\mathrm{C} 3$ & 12.93 & 12.41 & 12.04 & 12.29 & 12.22 \\
\hline $\mathrm{C} 4$ & 12.51 & 12.12 & 11.80 & 11.91 & 11.79 \\
\hline C5 & 10.77 & 10.90 & 10.92 & 10.80 & 10.92 \\
\hline $\mathrm{C} 6$ & 6.35 & 7.10 & 7.47 & 7.02 & 7.45 \\
\hline C7 & 8.07 & 8.19 & 8.19 & 8.03 & 8.02 \\
\hline $\mathrm{C} 8$ & 5.56 & 5.75 & 5.77 & 5.64 & 5.64 \\
\hline $\mathrm{Cg}$ & 3.78 & 3.97 & 4.01 & 3.89 & 3.90 \\
\hline $\mathrm{C} 10$ & 2.48 & 2.64 & 2.74 & 2.66 & 2.65 \\
\hline $\mathrm{C} 11$ & 1.82 & 1.86 & 1.94 & 1.89 & 1.77 \\
\hline $\mathrm{C} 12$ & 1.12 & 1.35 & 1.29 & 1.18 & 1.28 \\
\hline $\mathrm{C} 13$ & 0.76 & 0.82 & 0.87 & 0.83 & 0.82 \\
\hline $\mathrm{C} 14$ & 0.33 & 0.43 & 0.68 & 0.64 & 0.51 \\
\hline $\mathrm{C} 15$ & 0.28 & 0.15 & 0.34 & 0.31 & 0.20 \\
\hline alpha chain growth probability & 0.62 & 0.58 & 0.63 & 0.62 & 0.60 \\
\hline \multicolumn{6}{|c|}{ C1 - C50 estimated total product distribution, weight \% } \\
\hline $\mathrm{C} 1$ & 28.3 & 28.1 & 27.3 & 28.2 & 28.3 \\
\hline $\mathrm{C} 2-\mathrm{C}_{4}$ & 30.4 & 30.0 & 28.6 & 29.1 & 29.2 \\
\hline $\mathrm{C} 5-\mathrm{C} 12$ & 39.4 & 40.9 & 41.8 & 40.6 & 41.0 \\
\hline $\mathrm{C} 13-\mathrm{C} 50$ & 1.9 & 1.0 & 2.3 & 2.0 & 1.4 \\
\hline CO conversion, $\%$ & 5.4 & 6.6 & 7.2 & 6.8 & 5.9 \\
\hline rate, $\mathrm{g} \mathrm{CH} 2 / \mathrm{g} \mathrm{cat} / \mathrm{hr}$ & 0.35 & 0.42 & 0.47 & 0.44 & 0.38 \\
\hline $\mathrm{CO} 2$ formation, $\%$ & 0.3 & 0.2 & 0.2 & 0.2 & 0.2 \\
\hline
\end{tabular}


Time-on-Stream Plot for Co.055 - Run \#4

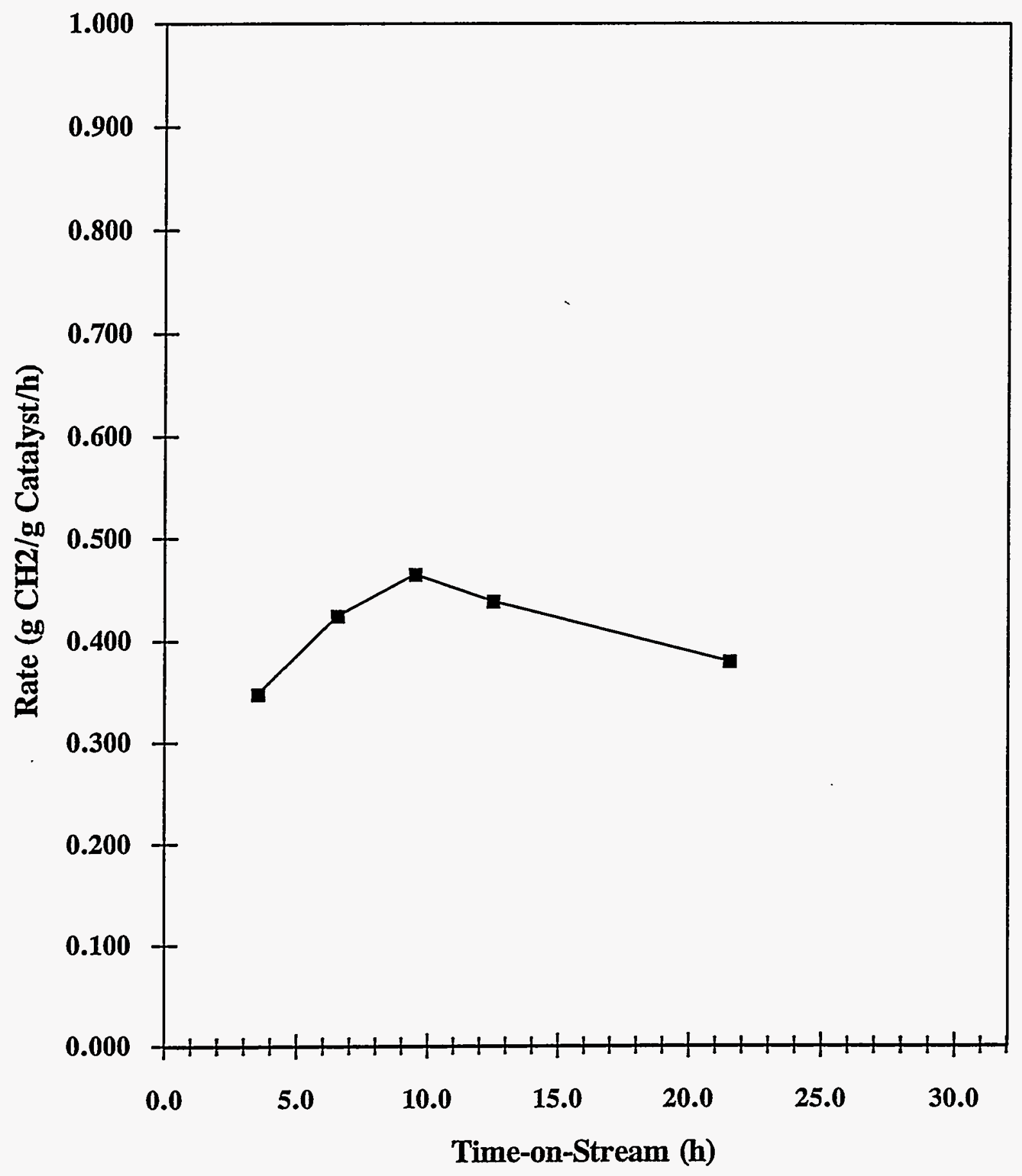




\section{Schulz-Flory Plot for Co.055 - Run \#4 \\ Time on Stream (hrs)}

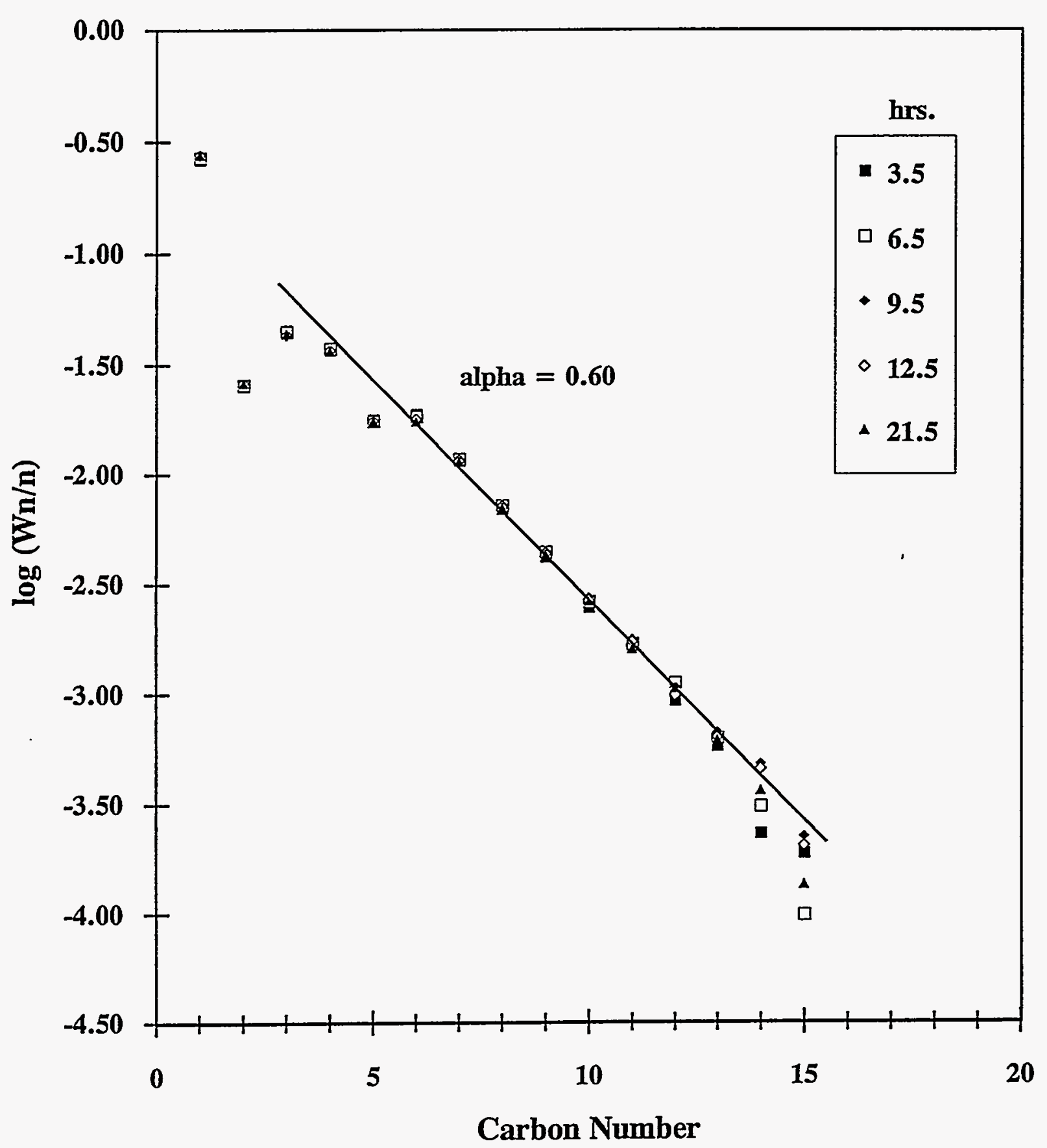




\section{Co.055 - Run \#5}

\begin{tabular}{|c|c|c|c|}
\hline Co wt\% & NM wt \% & Promotor wt\% & Support \\
\hline 20 & $\operatorname{Re} \quad 1.00$ & La2O3 1.00 & $\mathrm{Al2O3}$ \\
\hline
\end{tabular}

\section{SUMMARY REACTION DATA*}

Reaction Conditions:

$$
\begin{array}{ll}
\mathrm{P}=1.0 \mathrm{~atm} & \mathrm{CO} 2(\mathrm{~g} / \mathrm{g} \mathrm{cat} / \mathrm{hr})=0.030 \\
\mathrm{~T}=220^{\circ} \mathrm{C} & \mathrm{CO} 2(\% \text { of } \mathrm{CO})=0.1 \\
\mathrm{H} 2 / \mathrm{CO}=2 & \mathrm{O} / \mathrm{P}=1.83 \\
\text { weight of catalyst }=0.180 \mathrm{~g} & \\
\text { WHSV }=14.281 / \mathrm{hr} & \\
\text { time on stream }=24.5 \mathrm{hrs} &
\end{array}
$$

\begin{tabular}{||l|c||}
\hline CO conversion (\%) & 6.6 \\
\hline rate (g CH2/g cat/hr) & 0.41 \\
\hline alpha & 0.59 \\
\hline C1 (wt\%) & 29.6 \\
\hline C2 - C4 (wt\%) & 28.1 \\
\hline C5 - C12 (wt\%) & 41.1 \\
\hline C13 + (wt\%) & 1.2 \\
\hline
\end{tabular}

* Catalyst is directly reduced without calcination 


\section{Performance of Co.055}

\section{Dates: 11/07/94 - 11/08/94 Run \#5}

flow rate $=90.0 \mathrm{cc} / \mathrm{min}$, loading $=0.2 \mathrm{~g}, \mathrm{WHSV}=14.31 / \mathrm{hr}, \mathrm{H} 2 / \mathrm{CO}$ ratio in feed $=2$

\begin{tabular}{|c|c|c|c|c|c|c|}
\hline time on stream, hr & 0.5 & 3.5 & 6.5 & 9.5 & 12.5 & 15.5 \\
\hline reaction temperature, ${ }^{\circ} \mathrm{C}$ & 220 & 220 & 220 & 220 & 220 & 220 \\
\hline pressure, atm & 1.0 & 1.0 & 1.0 & 1.0 & 1.0 & 1.0 \\
\hline flow, cc/min & 90.0 & 90.0 & 90.0 & 90.0 & 90.0 & 90.0 \\
\hline \multicolumn{7}{|c|}{ C1 - C15 product distribution, weight \% } \\
\hline $\mathrm{C} 1$ & 27.20 & 28.68 & 28.15 & 28.62 & 28.72 & 28.88 \\
\hline $\mathrm{C} 2$ & 4.67 & 4.80 & 4.68 & 4.73 & 4.74 & 4.76 \\
\hline C3 & 11.75 & 11.75 & 11.42 & 11.44 & 11.45 & 11.48 \\
\hline $\mathrm{C} 4$ & 11.87 & 11.74 & 11.31 & 11.31 & 11.27 & 11.39 \\
\hline C5 & 11.18 & 10.77 & 10.56 & 10.50 & 10.52 & 10.53 \\
\hline C6 & 8.68 & 8.44 & 8.71 & 8.57 & 8.57 & 8.56 \\
\hline C7 & 8.28 & 7.91 & 7.76 & 7.71 & 7.66 & 7.61 \\
\hline C8 & 5.73 & 5.51 & 5.50 & 5.39 & 5.37 & 5.36 \\
\hline C9 & 3.94 & 3.78 & 3.82 & 3.80 & 3.80 & 3.81 \\
\hline $\mathrm{C} 10$ & 2.55 & 2.47 & 2.64 & 2.67 & 2.66 & 2.69 \\
\hline C11 & 2.12 & 1.84 & 1.86 & 1.83 & 1.85 & 1.86 \\
\hline $\mathrm{C} 12$ & 0.96 & 1.13 & 1.19 & 1.21 & 1.20 & 1.24 \\
\hline $\mathrm{C} 13$ & 0.69 & 0.71 & 0.84 & 0.78 & 0.77 & 0.73 \\
\hline C14 & 0.29 & 0.36 & 0.85 & 0.73 & 0.74 & 0.57 \\
\hline C15 & 0.10 & 0.12 & 0.70 & 0.70 & 0.65 & 0.54 \\
\hline alpha chain growth probability & 0.56 & 0.57 & 0.67 & 0.67 & 0.67 & 0.66 \\
\hline \multicolumn{7}{|c|}{ C1 - C50 estimated total product distribution, weight \% } \\
\hline $\mathrm{C} 1$ & 27.9 & 29.3 & 27.1 & 27.6 & 27.8 & 28.2 \\
\hline $\mathrm{C} 2-\mathrm{C} 4$ & 29.0 & 28.9 & 26.4 & 26.5 & 26.6 & 27.0 \\
\hline $\mathrm{C} 5-\mathrm{C} 12$ & 42.5 & 41.0 & 41.8 & 41.4 & 41.3 & 41.2 \\
\hline $\mathrm{C} 13-\mathrm{C} 50$ & 0.7 & 0.8 & 4.6 & 4.6 & 4.3 & 3.5 \\
\hline CO conversion, \% & 9.3 & 8.7 & 8.5 & 8.2 & 7.8 & 7.5 \\
\hline rate, $\mathrm{g} \mathrm{CH} 2 / \mathrm{g} \mathrm{cat} / \mathrm{hr}$ & 0.58 & 0.54 & 0.53 & 0.51 & 0.49 & 0.47 \\
\hline $\mathrm{CO} 2$ formation, $\%$ & 0.2 & 0.2 & 0.2 & 0.2 & 0.2 & 0.2 \\
\hline
\end{tabular}




\section{Performance of $\mathbf{C o . 0 5 5}$ \\ Dates: 11/07/94 - 11/08/94 Run \#5}

flow rate $=90.0 \mathrm{cc} / \mathrm{min}$, loading $=0.2 \mathrm{~g}, \mathrm{WHSV}=14.31 / \mathrm{hr}, \mathrm{H} 2 / \mathrm{CO}$ ratio in feed $=2$

\begin{tabular}{|c|c|c|c|}
\hline time on stream, hr & 18.5 & 21.5 & 24.5 \\
\hline reaction temperature, ${ }^{\circ} \mathrm{C}$ & 220 & 220 & 220 \\
\hline pressure, atm & 1.0 & 1.0 & 1.0 \\
\hline flow, $\mathrm{cc} / \mathrm{min}$ & 90.0 & 90.0 & 90.0 \\
\hline \multicolumn{4}{|c|}{ C1 - C15 product distribution, weight \% } \\
\hline $\mathrm{Cl}$ & 29.21 & 28.78 & 29.10 \\
\hline $\mathrm{C} 2$ & 4.81 & 4.75 & 4.80 \\
\hline C3 & 11.56 & 11.46 & 11.55 \\
\hline $\mathrm{C} 4$ & 11.09 & 11.15 & 11.20 \\
\hline C5 & 10.47 & 10.53 & 10.59 \\
\hline C6 & 8.44 & 8.56 & 8.60 \\
\hline C7 & 7.59 & 7.63 & 7.67 \\
\hline $\mathrm{C} 8$ & 5.34 & 5.36 & 5.39 \\
\hline C9 & 3.76 & 3.78 & 3.79 \\
\hline C10 & 2.66 & 2.70 & 2.66 \\
\hline $\mathrm{C} 11$ & 1.90 & 1.92 & 2.12 \\
\hline $\mathrm{C} 12$ & 1.24 & 1.21 & 1.35 \\
\hline $\mathrm{C} 13$ & 0.74 & 0.78 & 0.63 \\
\hline $\mathrm{C} 14$ & 0.64 & 0.71 & 0.37 \\
\hline $\mathrm{C} 15$ & 0.55 & 0.69 & 0.18 \\
\hline alpha chain growth probability & 0.66 & 0.67 & 0.59 \\
\hline \multicolumn{4}{|c|}{ C1 - C50 estimated total product distribution, weight $\%$} \\
\hline $\mathrm{Cl}$ & 28.6 & 27.8 & 29.6 \\
\hline $\mathrm{C} 2-\mathrm{C} 4$ & 26.9 & 26.4 & 28.1 \\
\hline C5 - C12 & 41.0 & 41.3 & 41.1 \\
\hline $\mathrm{C} 13-\mathrm{C} 50$ & 3.6 & 4.5 & 1.2 \\
\hline CO conversion, \% & 7.4 & 7.2 & 6.6 \\
\hline rate, $\mathrm{g} \mathrm{CH} 2 / \mathrm{g}$ cat $/ \mathrm{hr}$ & 0.46 & 0.45 & 0.41 \\
\hline $\mathrm{CO} 2$ formation, \% & 0.2 & 0.1 & 0.1 \\
\hline
\end{tabular}


Time-on-Stream Plot for Co.055 - Run \#5

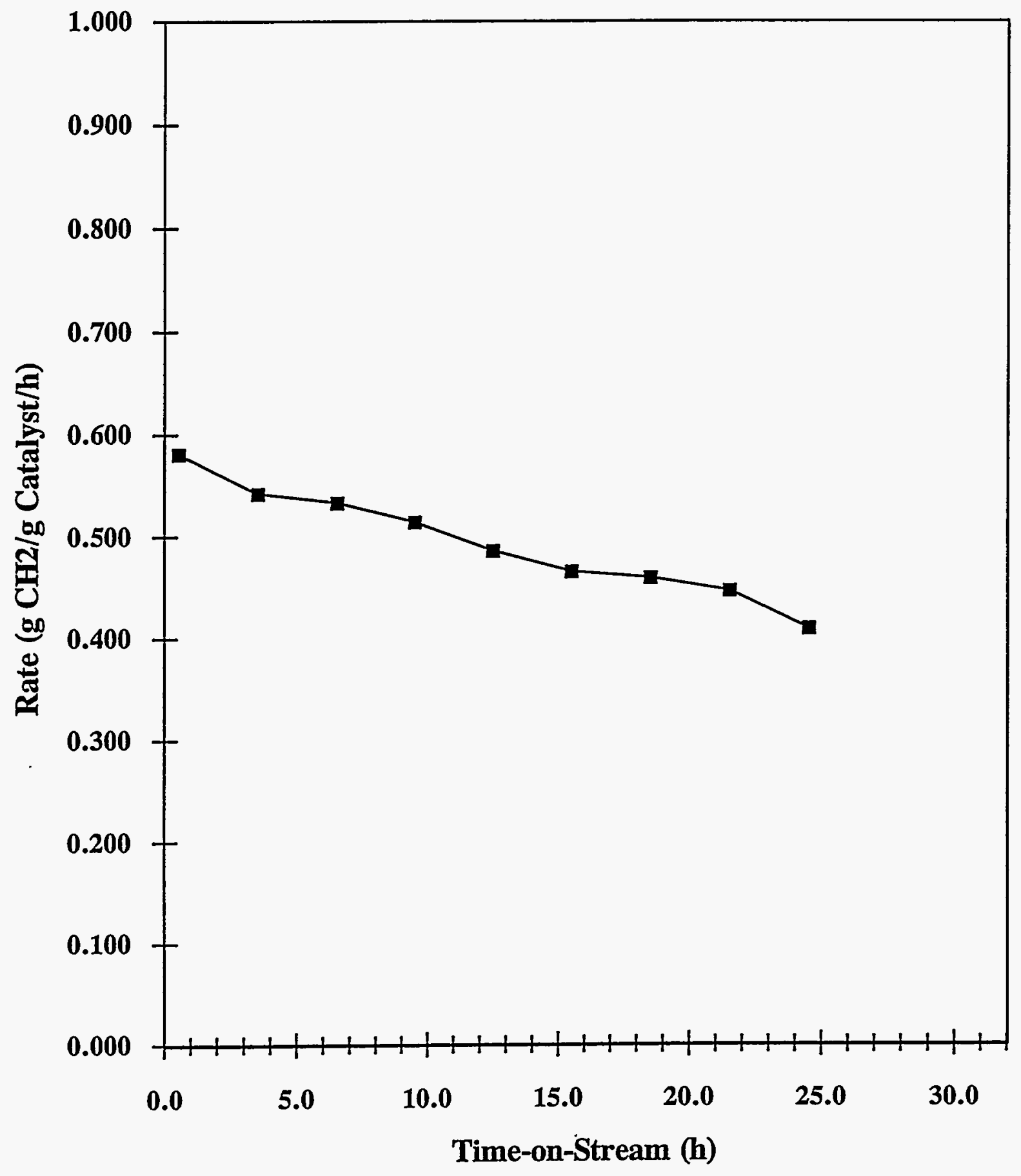


Schulz-Flory Plot for Co.055 - Run \#5

Time on Stream (hrs)

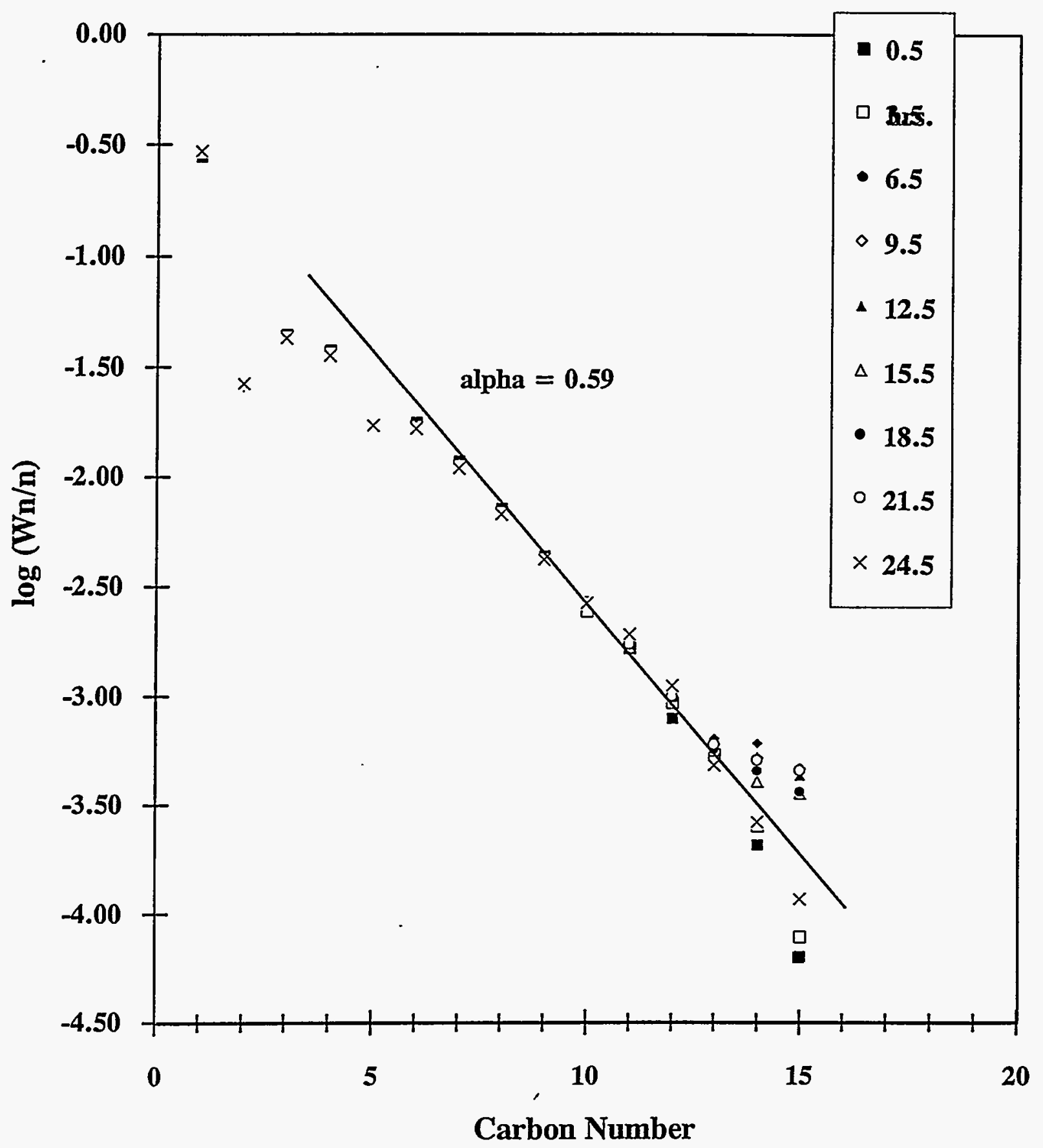




\section{Co.055 - Run \#6}

\begin{tabular}{|c|c|c|c|}
\hline Co wt\% & NM wt \% & Promotor wt\% & Support \\
\hline 20 & $\operatorname{Re} \quad 1.00$ & $\mathrm{La} 2 \mathrm{O3} \quad 1.00$ & $\mathrm{Al2O3}$ \\
\hline
\end{tabular}

\section{SUMMARY REACTION DATA*}

Reaction Conditions:

$$
\begin{array}{ll}
\mathrm{P}=1.0 \mathrm{~atm} & \mathrm{CO} 2(\mathrm{~g} / \mathrm{g} \text { cat } / \mathrm{hr})=0.023 \\
\mathrm{~T}=220^{\circ} \mathrm{C} & \mathrm{CO} 2(\% \text { of } \mathrm{CO})=0.1 \\
\mathrm{H} 2 / \mathrm{CO}=2 & \mathrm{O} / \mathrm{P}=1.21 \\
\text { weight of catalyst }=0.180 \mathrm{~g} & \\
\text { WHSV }=14.311 / \mathrm{hr} & \\
\text { time on stream }=27.5 \mathrm{hrs} &
\end{array}
$$

\begin{tabular}{||l|c|}
\hline \hline CO conversion (\%) & 7.5 \\
\hline rate (g CH2/g cat/hr) & 0.47 \\
\hline alpha & 0.63 \\
\hline $\mathrm{C} 1$ (wt\%) & 30.9 \\
\hline $\mathrm{C} 2-\mathrm{C} 4(\mathrm{wt} \%)$ & 29.1 \\
\hline $\mathrm{C} 5-\mathrm{C} 12(\mathrm{wt} \%)$ & 37.6 \\
\hline $\mathrm{C} 13+(\mathrm{wt} \%)$ & 2.4 \\
\hline
\end{tabular}

* Catalyst is reduced, calcined and rereduced before loading 


\section{Performance of Co.055}

Dates: 11/14/94 - 11/15/94 Run \#6

flow rate $=90.0 \mathrm{cc} / \mathrm{min}$, loading $=0.2 \mathrm{~g}, \mathrm{WHSV}=14.31 / \mathrm{hr}, \mathrm{H} 2 / \mathrm{CO}$ ratio in feed $=2$

\begin{tabular}{lc} 
time on stream, $\mathrm{hr}$ & 27.5 \\
reaction temperature, ${ }^{\circ} \mathrm{C}$ & 220 \\
pressure, atm & 1.0 \\
flow, cc/min & 90.0 \\
\hline
\end{tabular}

C1 - C15 product distribution, weight \%

C1

31.53

C2

4.98

C3

12.71

$\mathrm{C} 4$

12.09

C5

10.66

C6

7.91

C7

7.28

C8

4.68

C9

3.05

C10

1.94

C11

1.25

$\mathrm{C} 12$

0.67

C13

0.45

C14

0.40

C15

0.37

alpha chain growth probability

0.63

C1 - C50 estimated total product distribution, weight \%

C1

C2 - C4

30.9

C5 - C12

29.1

C13 - C50

37.6

2.4

CO conversion, \%

7.5

rate, $\mathrm{g} \mathrm{CH} 2 / \mathrm{g} \mathrm{cat} / \mathrm{hr}$

0.47

$\mathrm{CO} 2$ formation, $\%$

0.1 


\section{Performance of Co.055}

Dates: 11/14/94 - 11/15/94 Run \#6

flow rate $=90.0 \mathrm{cc} / \mathrm{min}$, loading $=0.2 \mathrm{~g}, \mathrm{WHSV}=14.31 / \mathrm{hr}, \mathrm{H} 2 / \mathrm{CO}$ ratio in feed $=2$

\begin{tabular}{|c|c|c|c|c|c|c|}
\hline time on stream, hr & 0.5 & 3.5 & 6.5 & 9.5 & 21.5 & 24.5 \\
\hline reaction temperature, ${ }^{\circ} \mathrm{C}$ & 220 & 220 & 220 & 220 & 220 & 220 \\
\hline pressure, atm & 1.0 & 1.0 & 1.0 & 1.0 & 1.0 & 1.0 \\
\hline flow, cc/min & 90.0 & 90.0 & 90.0 & 90.0 & 90.0 & 90.0 \\
\hline \multicolumn{7}{|c|}{ C1 - C15 product distribution, weight \% } \\
\hline $\mathrm{Cl}$ & 31.93 & 32.27 & 31.84 & 31.85 & 30.96 & 31.10 \\
\hline $\mathrm{C} 2$ & 5.23 & 5.21 & 5.13 & 5.11 & 4.93 & 4.94 \\
\hline $\mathrm{C} 3$ & 13.13 & 13.04 & 12.98 & 12.95 & 12.59 & 12.66 \\
\hline $\mathrm{C} 4$ & 12.73 & 12.68 & 12.60 & 12.59 & 12.01 & 12.10 \\
\hline $\mathrm{C5}$ & 11.25 & 11.12 & 11.12 & 11.08 & 10.84 & 10.77 \\
\hline C6 & 8.12 & 8.08 & 8.46 & 8.38 & 7.88 & 7.98 \\
\hline $\mathrm{C7}$ & 6.40 & 6.28 & 6.34 & 6.38 & 7.44 & 7.37 \\
\hline $\mathrm{C} 8$ & 4.18 & 4.09 & 4.14 & 4.18 & 4.76 & 4.74 \\
\hline C9 & 2.74 & 2.64 & 2.64 & 2.68 & 3.14 & 3.14 \\
\hline $\mathrm{C} 10$ & 1.64 & 1.71 & 1.84 & 1.84 & 2.18 & 1.97 \\
\hline C11 & 1.20 & 1.23 & 1.16 & 1.14 & 1.23 & 1.31 \\
\hline $\mathrm{C} 12$ & 0.73 & 0.92 & 0.64 & 0.73 & 0.82 & 0.71 \\
\hline C13 & 0.41 & 0.42 & 0.41 & 0.42 & 0.47 & 0.46 \\
\hline C14 & 0.23 & 0.24 & 0.35 & 0.33 & 0.38 & 0.39 \\
\hline C15 & 0.08 & 0.08 & 0.35 & 0.34 & 0.36 & 0.37 \\
\hline alpha chain growth probability & 0.55 & 0.55 & 0.63 & 0.63 & 0.63 & 0.63 \\
\hline \multicolumn{7}{|c|}{$\mathrm{C} 1$ - C50 estimated total product distribution, weight \% } \\
\hline $\mathrm{Cl}$ & 32.3 & 32.7 & 31.1 & 31.2 & 30.4 & 30.5 \\
\hline $\mathrm{C} 2-\mathrm{C} 4$ & 31.4 & 31.3 & 30.0 & 30.0 & 29.0 & 29.1 \\
\hline $\mathrm{C} 5-\mathrm{C} 12$ & 35.7 & 35.4 & 36.5 & 36.5 & 38.3 & 38.0 \\
\hline $\mathrm{C} 13-\mathrm{C} 50$ & 0.6 & 0.6 & 2.3 & 2.2 & 2.3 & 2.4 \\
\hline CO conversion, $\%$ & 9.1 & 8.3 & 7.9 & 7.5 & 7.3 & 7.3 \\
\hline rate, g CH2/g cat $/ \mathrm{hr}$ & 0.57 & 0.52 & 0.49 & 0.47 & 0.46 & 0.46 \\
\hline $\mathrm{CO} 2$ formation, \% & 0.2 & 0.2 & 0.2 & 0.2 & 0.2 & 0.1 \\
\hline
\end{tabular}


Time-on-Stream Plot for Co.055 - Run \#6

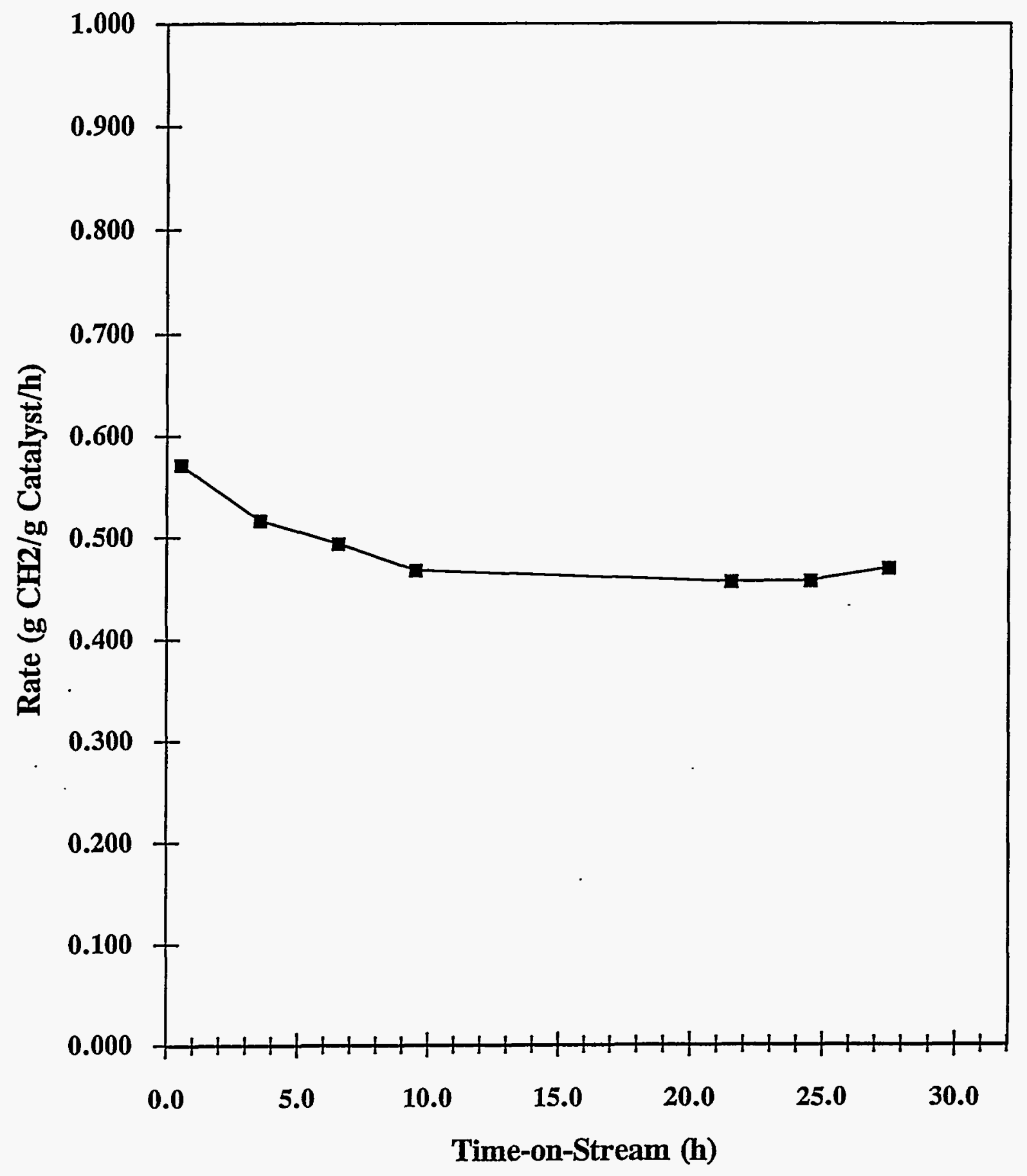


Schulz-Flory Plot for Co.055 - Run \#6

Time on Stream (hrs)

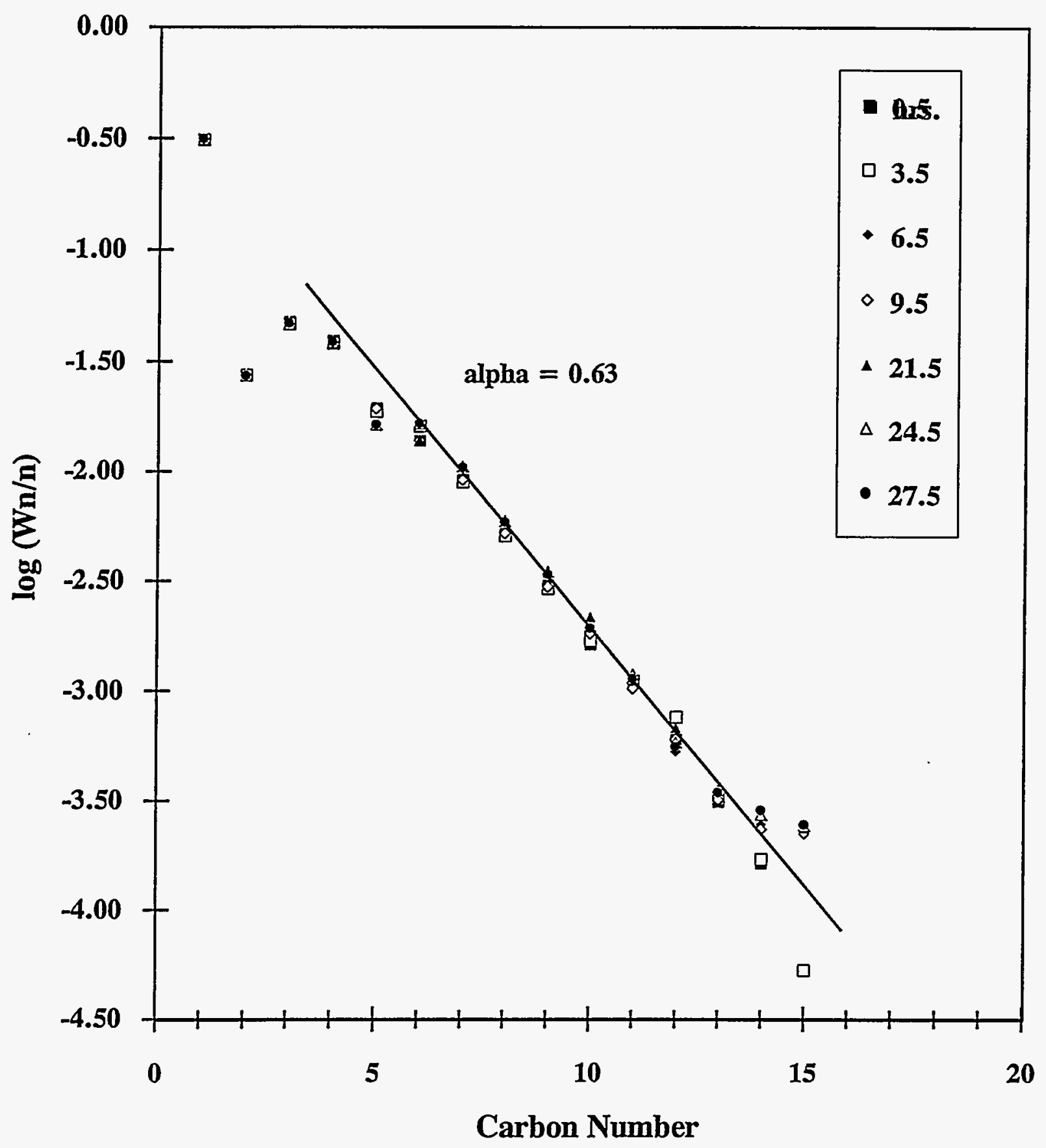




\section{Co.055 - Run \#7}

\begin{tabular}{|c|c|c|c|c|}
\hline Co wt\% & NM & wt \% & Promotor wt\% & Support \\
\hline 20 & $\mathbf{R e}$ & 1.00 & $\mathrm{La2O3} 1.00$ & $\mathrm{Al2O3}$ \\
\hline
\end{tabular}

\section{SUMMARY REACTION DATA*}

Reaction Conditions:

$$
\begin{array}{ll}
\mathrm{P}=1.0 \mathrm{~atm} & \mathrm{CO} 2(\mathrm{~g} / \mathrm{g} \mathrm{cat} / \mathrm{hr})=0.031 \\
\mathrm{~T}=220^{\circ} \mathrm{C} & \mathrm{CO} 2(\% \text { of CO})=0.1 \\
\mathrm{H} 2 / \mathrm{CO}=2 & \mathrm{O} / \mathrm{P}=1.11 \\
\text { weight of catalyst }=0.180 \mathrm{~g} & \\
\text { WHSV }=14.291 / \mathrm{hr} & \\
\text { time on stream }=24.5 \mathrm{hrs} &
\end{array}
$$

\begin{tabular}{||l|c|}
\hline CO conversion (\%) & 7.5 \\
\hline rate (g CH2/g cat/hr) & 0.47 \\
\hline alpha & 0.60 \\
\hline C1 (wt\%) & 33.5 \\
\hline $\mathrm{C} 2-\mathrm{C} 4(w t \%)$ & 31.1 \\
\hline $\mathrm{C} 5-\mathrm{C} 12(\mathrm{wt} \%)$ & 34.0 \\
\hline $\mathrm{C} 13+(\mathrm{wt} \%)$ & 1.4 \\
\hline
\end{tabular}

* Catalyst calcined in Nitrogen 


\section{Performance of Co.055 \\ Dates: 11/16/94 - 11/17/94 Run \#7}

flow rate $=90.0 \mathrm{cc} / \mathrm{min}$, loading $=0.2 \mathrm{~g}, \mathrm{WHSV}=14.31 / \mathrm{hr}, \mathrm{H} 2 / \mathrm{CO}$ ratio in feed $=2$

\begin{tabular}{|c|c|c|c|c|c|c|}
\hline time on stream, hr & 0.5 & 3.5 & 6.5 & 9.5 & 12.5 & 15.5 \\
\hline reaction temperature, ${ }^{\circ} \mathrm{C}$ & 220 & 220 & 220 & 220 & 220 & 220 \\
\hline pressure, atm & 1.0 & 1.0 & 1.0 & 1.0 & 1.0 & 1.0 \\
\hline flow, $\mathrm{cc} / \mathrm{min}$ & 90.0 & 90.0 & 90.0 & 90.0 & 90.0 & 90.0 \\
\hline \multicolumn{7}{|c|}{$\mathrm{C} 1$ - C15 product distribution, weight $\%$} \\
\hline $\mathrm{C} 1$ & 33.97 & 34.37 & 33.97 & 33.64 & 34.01 & 34.40 \\
\hline $\mathrm{C} 2$ & 5.47 & 5.48 & 5.37 & 5.30 & 5.34 & 5.37 \\
\hline C3 & 13.37 & 13.57 & 13.30 & 13.21 & 13.34 & 13.38 \\
\hline $\mathrm{C} 4$ & 12.84 & 13.11 & 12.71 & 12.67 & 12.59 & 12.67 \\
\hline $\mathrm{C} 5$ & 11.07 & 11.19 & 10.93 & 10.84 & 10.89 & 10.87 \\
\hline C6 & 7.71 & 7.91 & 7.92 & 7.88 & 7.86 & 7.78 \\
\hline C7 & 6.01 & 6.00 & 5.93 & 6.24 & 5.98 & 5.92 \\
\hline $\mathrm{C} 8$ & 3.78 & 3.76 & 3.72 & 3.97 & 3.75 & 3.74 \\
\hline $\mathrm{Cg}$ & 2.36 & 2.34 & 2.32 & 2.44 & 2.30 & 2.32 \\
\hline $\mathrm{C} 10$ & 1.37 & 0.02 & 1.60 & 1.66 & 1.72 & 1.38 \\
\hline $\mathrm{C} 11$ & 0.98 & 0.96 & 0.90 & 0.92 & 1.00 & 0.92 \\
\hline $\mathrm{C} 12$ & 0.48 & 0.47 & 0.57 & 0.46 & 0.46 & 0.46 \\
\hline $\mathrm{C} 13$ & 0.32 & 0.30 & 0.30 & 0.30 & 0.30 & 0.31 \\
\hline C14 & 0.18 & 0.26 & 0.25 & 0.24 & 0.21 & 0.24 \\
\hline $\mathrm{C} 15$ & 0.08 & 0.25 & 0.23 & 0.23 & 0.26 & 0.24 \\
\hline alpha chain growth probability & 0.55 & 0.61 & 0.60 & 0.61 & 0.61 & 0.60 \\
\hline \multicolumn{7}{|c|}{ C1 - C50 estimated total product distribution, weight \% } \\
\hline $\mathrm{C} 1$ & 34.1 & 33.8 & 33.5 & 33.1 & 33.4 & 33.9 \\
\hline $\mathrm{C} 2-\mathrm{C} 4$ & 31.8 & 31.6 & 30.9 & 30.7 & 30.7 & 30.9 \\
\hline $\mathrm{C} 5-\mathrm{C} 12$ & 33.5 & 32.9 & 34.0 & 34.6 & 34.2 & 33.6 \\
\hline $\mathrm{C} 13-\mathrm{C} 50$ & 0.6 & 1.6 & 1.5 & 1.5 & 1.7 & 1.6 \\
\hline CO conversion, \% & 9.8 & 8.8 & 8.5 & 8.3 & 8.0 & 7.9 \\
\hline rate, g CH2/g cat/hr & 0.61 & 0.55 & 0.53 & 0.52 & 0.50 & 0.49 \\
\hline $\mathrm{CO} 2$ formation, \% & 0.2 & 0.2 & 0.2 & 0.2 & 0.1 & 0.1 \\
\hline
\end{tabular}




\section{Performance of Co.055}

Dates: 11/16/94 - 11/17/94 Run \#7

\begin{tabular}{|c|c|c|c|}
\hline time on stream, hr & 18.5 & 21.5 & 24.5 \\
\hline reaction temperature, ${ }^{\circ} \mathrm{C}$ & 220 & 220 & 220 \\
\hline pressure, atm & 1.0 & 1.0 & 1.0 \\
\hline flow, cc/min & 90.0 & 90.0 & 90.0 \\
\hline \multicolumn{4}{|c|}{ C1 - C15 product distribution, weight \% } \\
\hline $\mathrm{C} 1$ & 34.16 & 33.77 & 33.94 \\
\hline $\mathrm{C} 2$ & 5.34 & 5.29 & 5.30 \\
\hline $\mathrm{C} 3$ & 13.44 & 13.44 & 13.50 \\
\hline $\mathrm{C} 4$ & 12.71 & 12.72 & 12.73 \\
\hline $\mathrm{C5}$ & 10.98 & 11.05 & 11.02 \\
\hline C6 & 7.81 & 7.96 & 7.95 \\
\hline C7 & 5.99 & 6.04 & 6.03 \\
\hline $\mathrm{C} 8$ & 3.76 & 3.75 & 3.79 \\
\hline $\mathrm{Cg}$ & 2.29 & 2.32 & 2.29 \\
\hline $\mathrm{C} 10$ & 1.37 & 1.58 & 1.39 \\
\hline $\mathrm{C} 11$ & 0.91 & 0.81 & 0.91 \\
\hline $\mathrm{C} 12$ & 0.52 & 0.53 & 0.46 \\
\hline $\mathrm{C} 13$ & 0.28 & 0.27 & 0.27 \\
\hline C14 & 0.23 & 0.23 & 0.23 \\
\hline $\mathrm{C} 15$ & 0.21 & 0.23 & 0.20 \\
\hline alpha chain growth probability & 0.60 & 0.60 & 0.60 \\
\hline
\end{tabular}

C1 - C50 estimated total product distribution, weight \%

$\begin{array}{lccc}\mathrm{C} 1 & 33.7 & 33.2 & 33.5 \\ \mathrm{C} 2-\mathrm{C} 4 & 31.1 & 31.0 & 31.1 \\ \mathrm{C} 5-\mathrm{C} 12 & 33.8 & 34.3 & 34.0 \\ \mathrm{C} 13-\mathrm{C} 50 & 1.4 & 1.5 & 1.4\end{array}$

CO conversion, \% rate, $\mathrm{g} \mathrm{CH} 2 / \mathrm{g} \mathrm{cat} / \mathrm{hr}$

$\begin{array}{lll}7.7 & 7.6 & 7.5\end{array}$

$\begin{array}{lll}0.48 & 0.47 & 0.47\end{array}$

$\mathrm{CO} 2$ formation, \%

$\begin{array}{lll}0.2 & 0.2 & 0.1\end{array}$


Time-on-Stream Plot for Co.055 - Run \#7

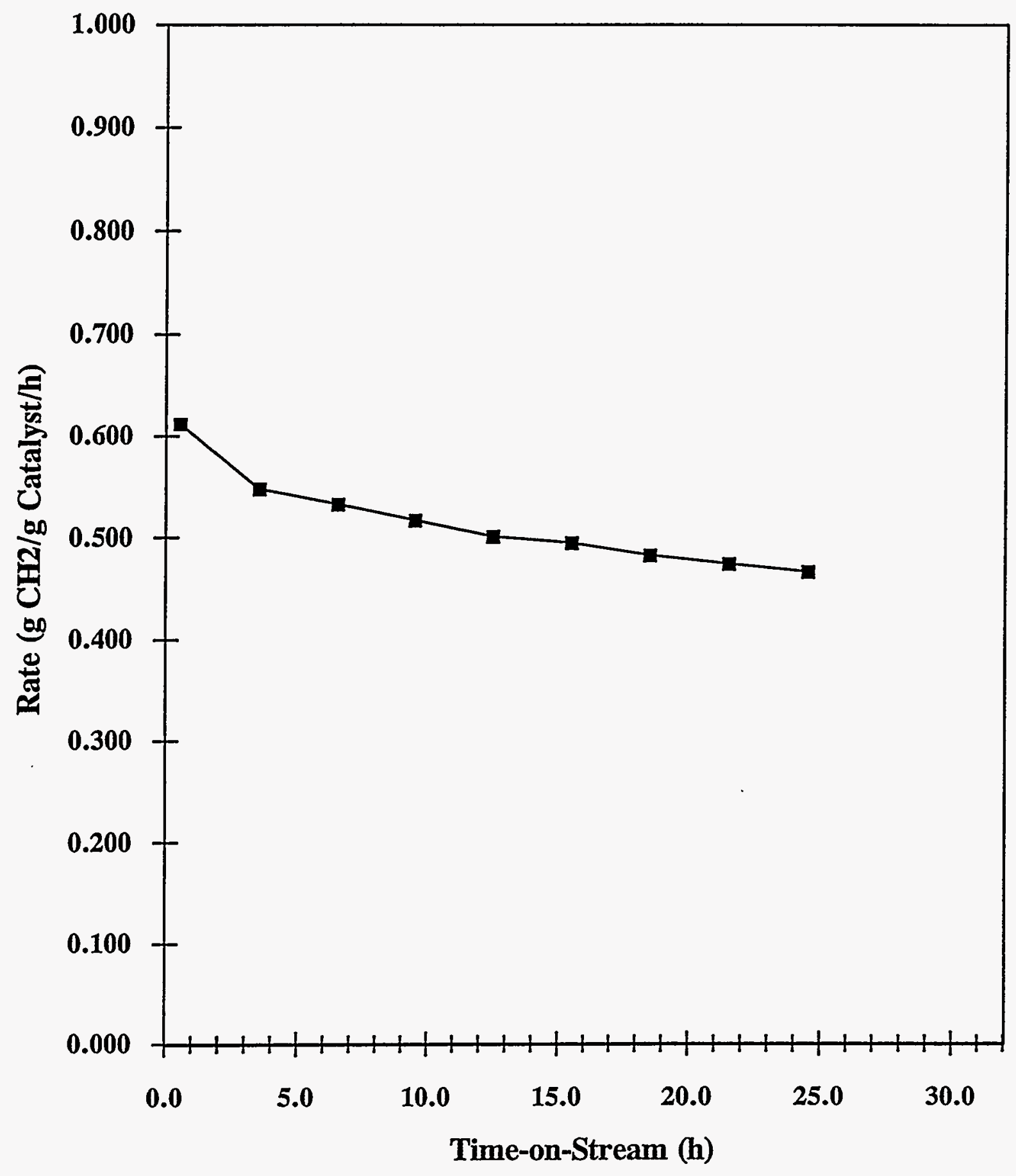




\section{Schulz-Flory Plot for Co.055 - Run \#7 \\ Time on Stream (hrs)}

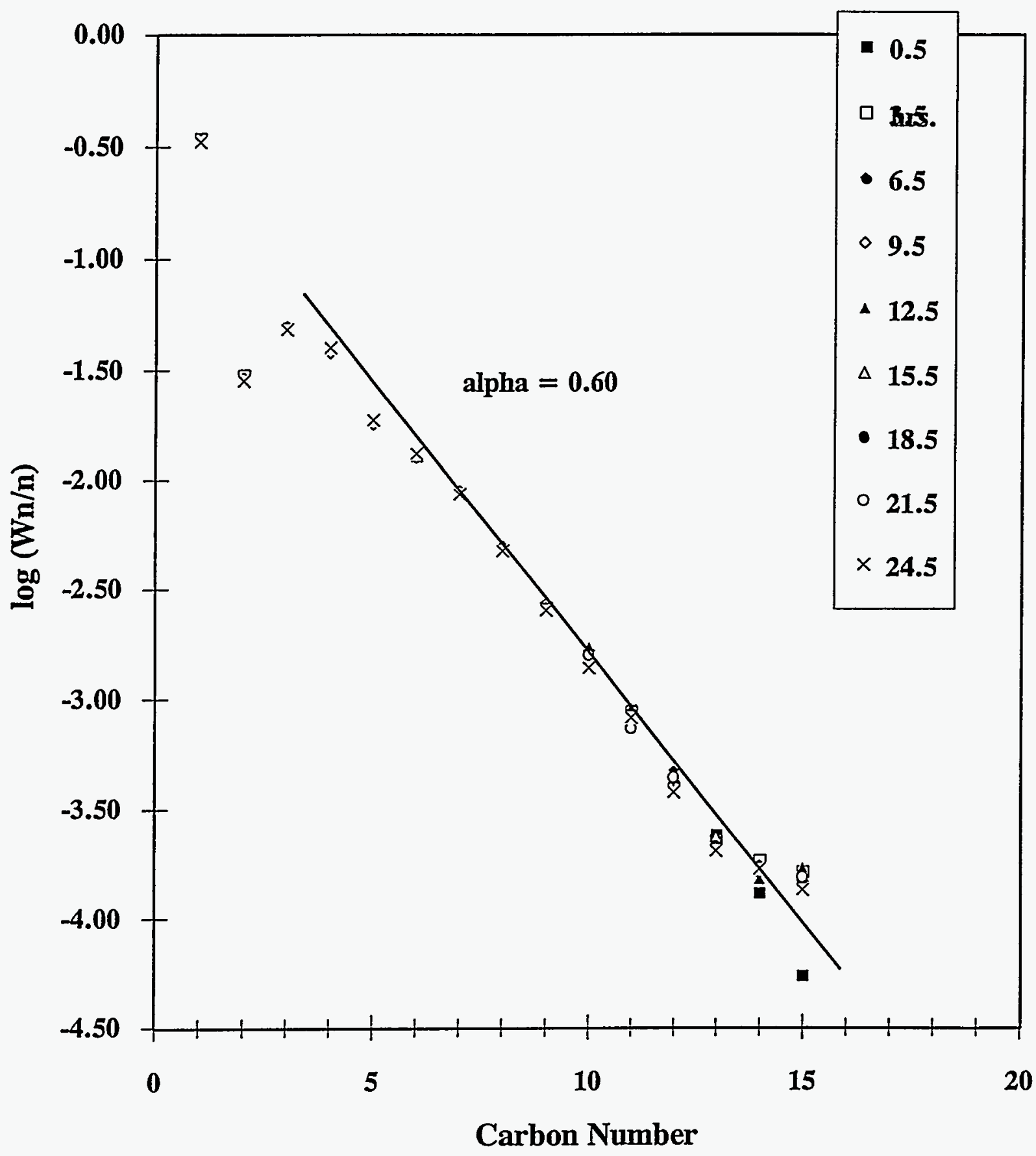




\section{Co.056 - Run \#1}

\begin{tabular}{||c||cc||c|c||c|}
\hline \hline Co wt\% & NM wt \% & \multicolumn{3}{|c||}{ Promotor wt\% } & Support \\
\hline 20 & & La & 8.50 & & SiO2 \\
\hline
\end{tabular}

\section{SUMMARY REACTION DATA}

Reaction Conditions:

$$
\begin{array}{ll}
\mathrm{P}=1.0 \mathrm{~atm} & \mathrm{CO} 2(\mathrm{~g} / \mathrm{g} \mathrm{cat} / \mathrm{hr})=0.029 \\
\mathrm{~T}=220^{\circ} \mathrm{C} & \mathrm{CO} 2(\% \text { of CO})=0.1 \\
\mathrm{H} 2 / \mathrm{CO}=2 & \mathrm{O} / \mathrm{P}=8.22 \\
\text { weight of catalyst }=0.200 \mathrm{~g} & \\
\text { WHSV }=12.881 / \mathrm{hr} & \\
\text { time on stream }=27.5 \mathrm{hrs} &
\end{array}
$$

\begin{tabular}{||l|c|}
\hline \hline CO conversion (\%) & 2.4 \\
\hline rate (g CH2/g cat/hr) & 0.14 \\
\hline alpha & 0.69 \\
\hline C1 (wt\%) & 23.9 \\
\hline C2 - C4 (wt\%) & 27.2 \\
\hline C5 - C12 (wt\%) & 43.3 \\
\hline C13 $+(w t \%)$ & 5.6 \\
\hline
\end{tabular}




\section{Performance of Co.056 \\ Dates: 10/07/94 - 10/08/94 Run \#1}

flow rate $=90.0 \mathrm{cc} / \mathrm{min}$, loading $=0.2 \mathrm{~g}, \mathrm{WHSV}=12.91 / \mathrm{hr}, \mathrm{H} 2 / \mathrm{CO}$ ratio in feed $=2$

\begin{tabular}{lcccccc}
\hline & & & & & & \\
time on stream, hr & 0.5 & 3.5 & 6.5 & 12.5 & 15.5 & 18.5 \\
reaction temperature, ${ }^{\circ} \mathrm{C}$ & 220 & 220 & 220 & 220 & 220 & 220 \\
pressure, atm & 1.0 & 1.0 & 1.0 & 1.0 & 1.0 & 1.0 \\
flow, cc/min & 90.0 & 90.0 & 90.0 & 90.0 & 90.0 & 90.0 \\
\hline
\end{tabular}

C1 - C15 product distribution, weight \%

\begin{tabular}{|c|c|c|c|c|c|c|}
\hline $\mathrm{Cl}$ & 18.31 & 22.20 & 21.64 & 23.61 & 24.59 & 23.75 \\
\hline $\mathrm{C} 2$ & 4.51 & 5.60 & 5.39 & 5.83 & 6.05 & 5.86 \\
\hline C3 & 9.98 & 11.03 & 10.33 & 10.88 & 11.22 & 10.80 \\
\hline $\mathrm{C} 4$ & 10.91 & 11.28 & 10.63 & 10.92 & 11.16 & 10.77 \\
\hline $\mathrm{C} 5$ & 11.98 & 11.41 & 11.38 & 11.19 & 10.57 & 11.27 \\
\hline C6 & 10.24 & 6.59 & 9.02 & 7.99 & 7.99 & 8.79 \\
\hline $\mathrm{C7}$ & 8.97 & 8.50 & 8.43 & 8.08 & 7.85 & 8.00 \\
\hline $\mathrm{C} 8$ & 6.81 & 6.51 & 6.41 & 6.11 & 5.88 & 5.98 \\
\hline $\mathrm{C} 9$ & 5.16 & 4.93 & 4.81 & 4.43 & 4.36 & 4.39 \\
\hline $\mathrm{C} 10$ & 3.94 & 3.79 & 3.78 & 3.56 & 3.33 & 3.31 \\
\hline $\mathrm{C} 11$ & 2.83 & 2.71 & 2.57 & 2.38 & 2.24 & 2.27 \\
\hline $\mathrm{C} 12$ & 2.05 & 1.92 & 1.98 & 1.72 & 1.69 & 1.70 \\
\hline $\mathrm{C} 13$ & 1.66 & 1.54 & 1.39 & 1.33 & 1.24 & 1.19 \\
\hline $\mathrm{C} 14$ & 1.40 & 1.24 & 1.20 & 1.07 & 0.98 & 1.02 \\
\hline C15 & 1.25 & 0.75 & 1.04 & 0.91 & 0.84 & 0.89 \\
\hline alpha chain growth probability & 0.72 & 0.69 & 0.71 & 0.70 & 0.69 & 0.70 \\
\hline
\end{tabular}

C1 - C50 estimated total product distribution, weight \%

\begin{tabular}{lcccccc}
$\mathrm{C} 1$ & 17.3 & 21.9 & 20.7 & 22.7 & 23.8 & 22.8 \\
$\mathrm{C} 2-\mathrm{C} 4$ & 24.0 & 27.5 & 25.2 & 26.6 & 27.5 & 26.4 \\
$\mathrm{C} 5-\mathrm{C} 12$ & 50.3 & 45.5 & 47.1 & 44.6 & 43.2 & 44.9 \\
$\mathrm{C} 13-\mathrm{C} 50$ & 8.4 & 5.1 & 7.0 & 6.0 & 5.6 & 5.9 \\
& & & & & & \\
\hline CO conversion, \% & 4.7 & 2.4 & 3.1 & 2.7 & 2.6 & 2.6 \\
rate, g CH2/g cat/hr & 0.26 & 0.13 & 0.17 & 0.15 & 0.15 & 0.15 \\
$\mathrm{CO}$ formation, \% & 0.2 & 0.3 & 0.2 & 0.2 & 0.2 & 0.2 \\
& & & & & & \\
\hline
\end{tabular}




\section{Performance of $\mathbf{C o . 0 5 6}$ \\ Dates: 10/07/94 - 10/08/94 Run \#1}

flow rate $=90.0 \mathrm{cc} / \mathrm{min}$, loading $=0.2 \mathrm{~g}$, WHSV $=12.9 \mathrm{1} / \mathrm{hr}, \mathrm{H} 2 / \mathrm{CO}$ ratio in feed $=2$

\begin{tabular}{|c|c|c|c|}
\hline time on stream, hr & 21.5 & 24.5 & 27.5 \\
\hline reaction temperature, ${ }^{\circ} \mathrm{C}$ & 220 & 220 & 220 \\
\hline pressure, atm & 1.0 & 1.0 & 1.0 \\
\hline flow, cc/min & 90.0 & 90.0 & 90.0 \\
\hline \multicolumn{4}{|c|}{$\mathrm{C} 1-\mathrm{C} 15$ product distribution, weight $\%$} \\
\hline $\mathrm{C} 1$ & 23.95 & 25.31 & 24.84 \\
\hline $\mathrm{C} 2$ & 5.94 & 6.25 & 6.15 \\
\hline C3 & 10.90 & 11.44 & 11.16 \\
\hline $\mathrm{C} 4$ & 10.83 & 11.26 & 11.00 \\
\hline $\mathrm{C} 5$ & 11.30 & 10.82 & 11.24 \\
\hline $\mathrm{C} 6$ & 9.03 & 8.00 & 7.95 \\
\hline $\mathrm{C7}$ & 7.87 & 7.60 & 7.88 \\
\hline $\mathrm{C} 8$ & 5.86 & 5.60 & 5.79 \\
\hline $\mathrm{C} 9$ & 4.24 & 4.05 & 4.18 \\
\hline $\mathrm{C} 10$ & 3.22 & 3.10 & 3.18 \\
\hline C11 & 2.15 & 2.08 & 2.16 \\
\hline $\mathrm{C} 12$ & 1.61 & 1.53 & 1.55 \\
\hline $\mathrm{C} 13$ & 1.21 & 1.16 & 1.11 \\
\hline C14 & 0.99 & 0.95 & 0.97 \\
\hline $\mathrm{C} 15$ & 0.90 & 0.85 & 0.85 \\
\hline alpha chain growth probability & 0.70 & 0.69 & 0.69 \\
\hline \multicolumn{4}{|c|}{ C1 - C50 estimated total product distribution, weight $\%$} \\
\hline $\mathrm{Cl}$ & 23.0 & 24.3 & 23.9 \\
\hline $\mathrm{C} 2-\mathrm{C} 4$ & 26.5 & 27.8 & 27.2 \\
\hline $\mathrm{C} 5-\mathrm{C} 12$ & 44.6 & 42.2 & 43.3 \\
\hline $\mathrm{C} 13-\mathrm{C} 50$ & 6.0 & 5.6 & 5.6 \\
\hline CO conversion, \% & 2.6 & 2.5 & 2.4 \\
\hline rate, g CH2/g cat $/ \mathrm{hr}$ & 0.15 & 0.14 & 0.14 \\
\hline $\mathrm{CO} 2$ formation, \% & 0.2 & 0.2 & 0.1 \\
\hline
\end{tabular}


Time-on-Stream Plot for Co.056 - Run \#1

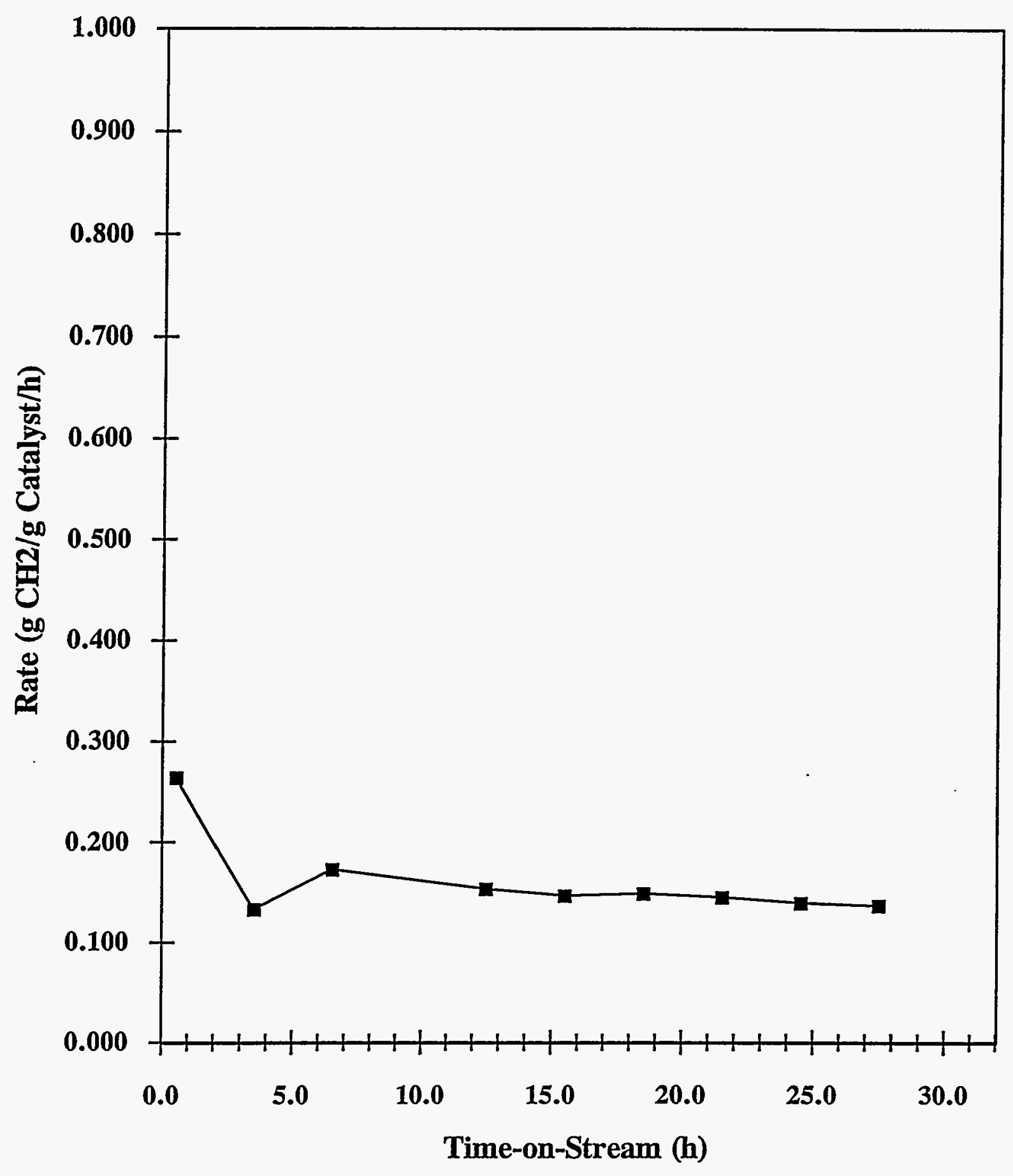


Schulz-Flory Plot for Co.056 - Run \#1

Time on Stream (hrs)

hrs.

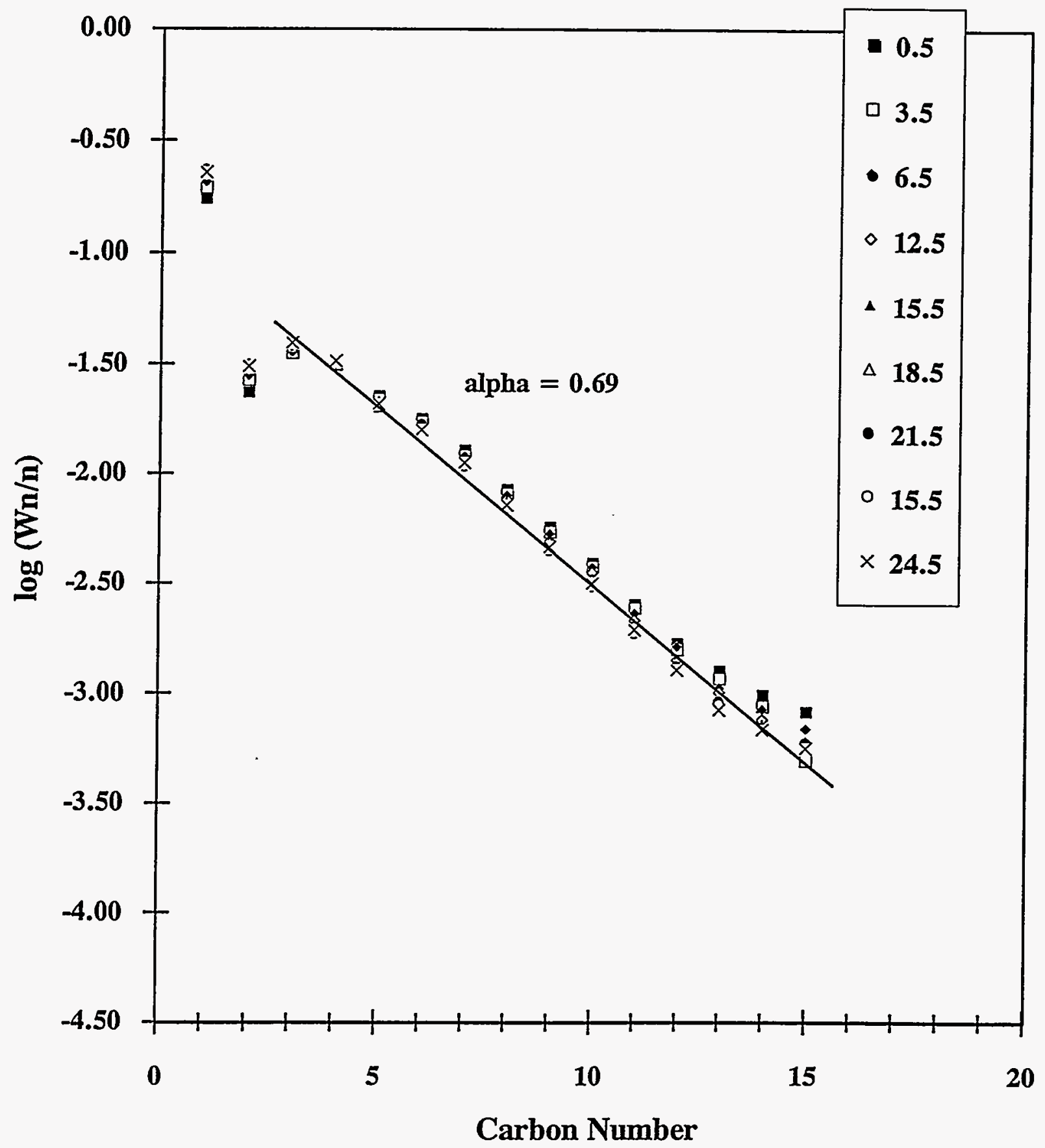




\section{Co.056 - Run \#2}

\begin{tabular}{||c||cc||c|c|c|}
\hline \hline Co wt\% & NM wt \% & \multicolumn{3}{|c|}{ Promotor wt\% } & Support \\
\hline 20 & & La & 8.50 & & SiO2 \\
\hline
\end{tabular}

\section{SUMMARY REACTION DATA*}

Reaction Conditions:

$$
\begin{array}{ll}
\mathrm{P}=1.0 \mathrm{~atm} & \mathrm{CO} 2(\mathrm{~g} / \mathrm{g} \mathrm{cat} / \mathrm{hr})=0.033 \\
\mathrm{~T}=220^{\circ} \mathrm{C} & \mathrm{CO} 2(\% \text { of CO})=0.2 \\
\mathrm{H} 2 / \mathrm{CO}=2 & \mathrm{O} / \mathrm{P}=9.44 \\
\text { weight of catalyst }=0.184 \mathrm{~g} & \\
\text { WHSV }=13.941 / \mathrm{hr} & \\
\text { time on stream }=21.5 \mathrm{hrs} &
\end{array}
$$

\begin{tabular}{|l|c|}
\hline CO conversion (\%) & 2.2 \\
\hline rate (g CH2/g cat/hr) & 0.14 \\
\hline alpha & 0.68 \\
\hline C1 (wt\%) & 23.4 \\
\hline C2 - C4 (wt\%) & 27.3 \\
\hline C5 - C12 (wt\%) & 44.7 \\
\hline C13 + (wt\%) & 4.7 \\
\hline
\end{tabular}

* Catalyst is directly reduced without calcination 


\title{
Performance of Co.056
}

\author{
Dates: 11/09/94 - 11/10/94 Run \#2
}

flow rate $=90.0 \mathrm{cc} / \mathrm{min}$, loading $=0.2 \mathrm{~g}, \mathrm{WHSV}=13.91 / \mathrm{hr}, \mathrm{H} 2 / \mathrm{CO}$ ratio in feed $=2$

\begin{tabular}{|c|c|c|c|c|c|c|}
\hline time on stream, hr & 0.5 & 3.5 & 6.5 & 9.5 & 12.5 & 15.5 \\
\hline reaction temperature, ${ }^{\circ} \mathrm{C}$ & 220 & 220 & 220 & 220 & 220 & 220 \\
\hline pressure, atm & 1.0 & 1.0 & 1.0 & 1.0 & 1.0 & 1.0 \\
\hline flow, cc/min & 90.0 & 90.0 & 90.0 & 90.0 & 90.0 & 90.0 \\
\hline \multicolumn{7}{|c|}{ C1 - C15 product distribution, weight \% } \\
\hline $\mathrm{C} 1$ & 18.82 & 20.53 & 21.03 & 21.86 & 23.29 & 22.98 \\
\hline $\mathrm{C} 2$ & 4.76 & 5.20 & 5.29 & 5.48 & 5.85 & 5.76 \\
\hline C3 & 10.26 & 10.24 & 10.12 & 10.30 & 10.86 & 10.66 \\
\hline $\mathrm{C} 4$ & 11.10 & 10.36 & 10.37 & 10.42 & 11.00 & 10.69 \\
\hline C5 & 12.24 & 11.51 & 11.08 & 11.12 & 11.62 & 11.18 \\
\hline C6 & 10.01 & 8.14 & 9.33 & 9.25 & 8.71 & 8.30 \\
\hline C7 & 9.48 & 8.85 & 8.43 & 8.39 & 6.64 & 8.18 \\
\hline $\mathrm{C} 8$ & 7.26 & 6.78 & 6.42 & 6.36 & 1.80 & 6.16 \\
\hline C9 & 5.48 & 5.10 & 4.82 & 4.81 & 4.70 & 4.55 \\
\hline $\mathrm{C} 10$ & 3.85 & 3.59 & 3.74 & 3.68 & 4.84 & 3.75 \\
\hline $\mathrm{C} 11$ & 2.97 & 3.24 & 2.58 & 2.51 & 3.45 & 2.39 \\
\hline C12 & 1.95 & 2.14 & 1.94 & 1.97 & 2.59 & 1.70 \\
\hline $\mathrm{C} 13$ & 1.15 & 1.84 & 1.74 & 1.38 & 1.87 & 1.28 \\
\hline C14 & 0.50 & 1.63 & 1.65 & 1.28 & 1.55 & 1.21 \\
\hline C15 & 0.17 & 0.86 & 1.46 & 1.20 & 1.21 & 1.21 \\
\hline alpha chain growth probability & 0.59 & 0.70 & 0.73 & 0.72 & 0.71 & 0.72 \\
\hline \multicolumn{7}{|c|}{ C1 - C50 estimated total product distribution, weight $\%$} \\
\hline C1 & 19.6 & 20.3 & 19.5 & 20.6 & 22.4 & 21.5 \\
\hline $\mathrm{C} 2-\mathrm{C} 4$ & 27.2 & 25.5 & 23.9 & 24.7 & 26.6 & 25.4 \\
\hline $\mathrm{C} 5-\mathrm{C} 12$ & 52.0 & 48.2 & 46.8 & 46.7 & 42.7 & 45.1 \\
\hline $\mathrm{C} 13-\mathrm{C} 50$ & 1.2 & 5.9 & 9.8 & 8.0 & 8.3 & 8.1 \\
\hline CO conversion, \% & 3.3 & 2.9 & 2.9 & 2.6 & 2.4 & 2.5 \\
\hline rate, $\mathrm{g} \mathrm{CH} 2 / \mathrm{g} \mathrm{cat} / \mathrm{hr}$ & 0.20 & 0.18 & 0.17 & 0.16 & 0.14 & 0.15 \\
\hline $\mathrm{CO} 2$ formation, \% & 0.2 & 0.1 & 0.1 & 0.1 & 0.1 & 0.1 \\
\hline
\end{tabular}




\section{Performance of Co.056}

\section{Dates: 11/09/94 - 11/10/94 Run \#2}

flow rate $=90.0 \mathrm{cc} / \mathrm{min}$, loading $=0.2 \mathrm{~g}, \mathrm{WHSV}=13.9 \mathrm{1} / \mathrm{hr}, \mathrm{H} 2 / \mathrm{CO}$ ratio in feed $=2$

\begin{tabular}{lcc}
\hline time on stream, hr & 18.5 & 21.5 \\
reaction temperature, ${ }^{\circ} \mathrm{C}$ & 220 & 220 \\
pressure, atm & 1.0 & 1.0 \\
flow, cc/min & 90.0 & 90.0 \\
\hline C1 - C15 product distribution, weight \% & & \\
& & \\
C1 & & \\
& 23.48 & 23.87 \\
C2 & & \\
C3 & 5.90 & 6.00 \\
C4 & 10.88 & 10.97 \\
& 10.70 & 10.89 \\
C5 & & \\
C6 & 11.26 & 11.37 \\
C7 & 8.15 & 8.49 \\
C8 & 8.19 & 8.15 \\
C9 & 6.04 & 6.00 \\
C10 & 4.47 & 4.34 \\
C11 & 3.37 & 3.08 \\
C12 & 2.26 & 2.42 \\
C13 & 1.77 & 1.66 \\
C14 & 1.33 & 1.10 \\
C15 & 1.22 & 0.96 \\
& 0.98 & 0.70 \\
alpha & chain growth probability & \\
& 0.70 & 0.68 \\
\hline
\end{tabular}

C1 - C50 estimated total product distribution, weight \%

$\begin{array}{lcc}\text { C1 } & 22.5 & 23.4 \\ \text { C2 - C4 } & 26.3 & 27.3 \\ \text { C5 - C12 } & 44.7 & 44.7 \\ \text { C13 - C50 } & 6.5 & 4.7\end{array}$

CO conversion, $\%$

rate, $\mathrm{g} \mathrm{CH} 2 / \mathrm{g} \mathrm{cat} / \mathrm{hr}$

$\mathrm{CO} 2$ formation, \% $\begin{array}{ll}2.3 & 2.2\end{array}$

$0.14 \quad 0.14$

$0.1 \quad 0.2$ 
Time-on-Stream Plot for Co.056 - Run \#2

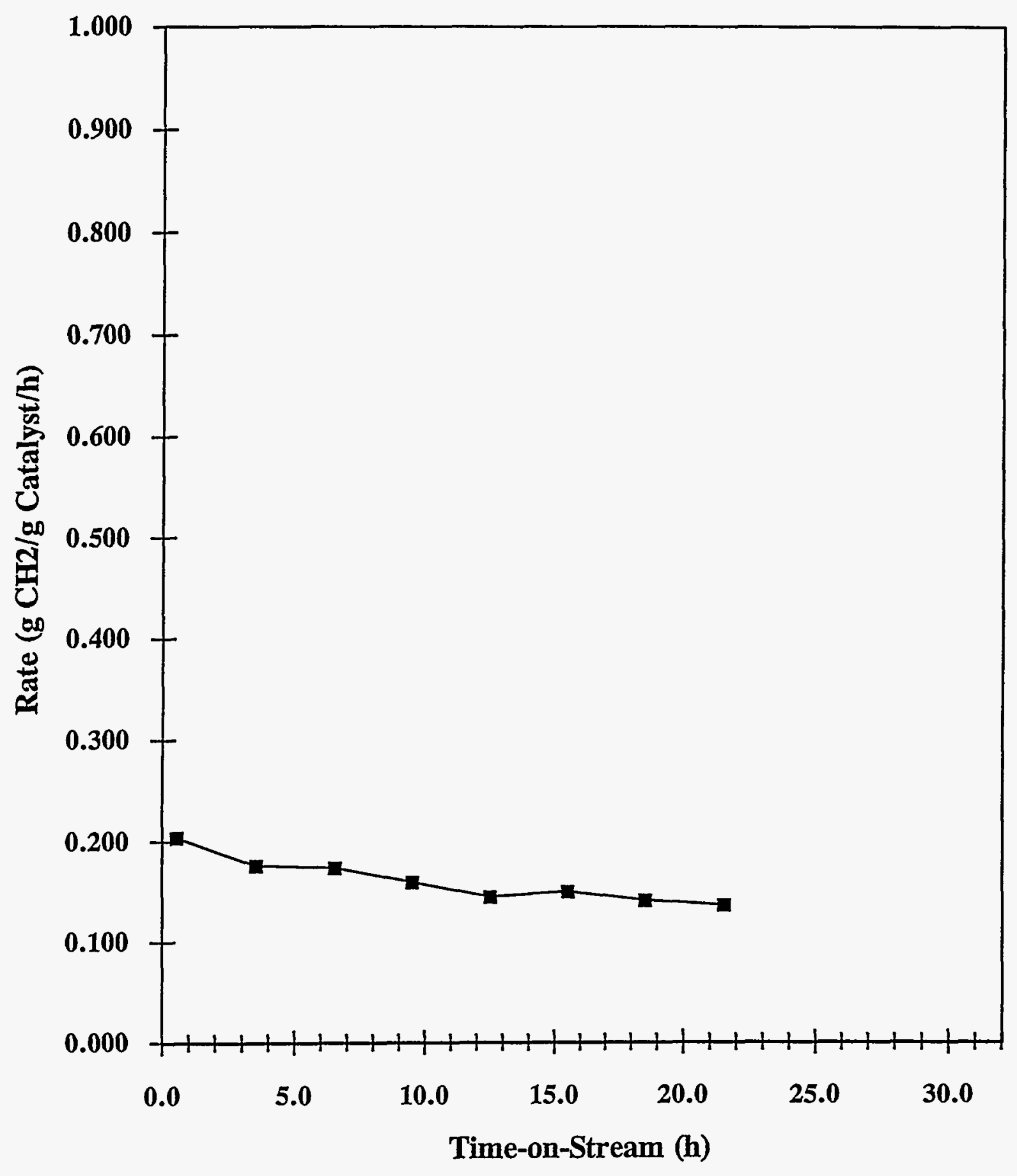


Schulz-Flory Plot for Co.056 - Run \#2

Time on Stream (hrs)

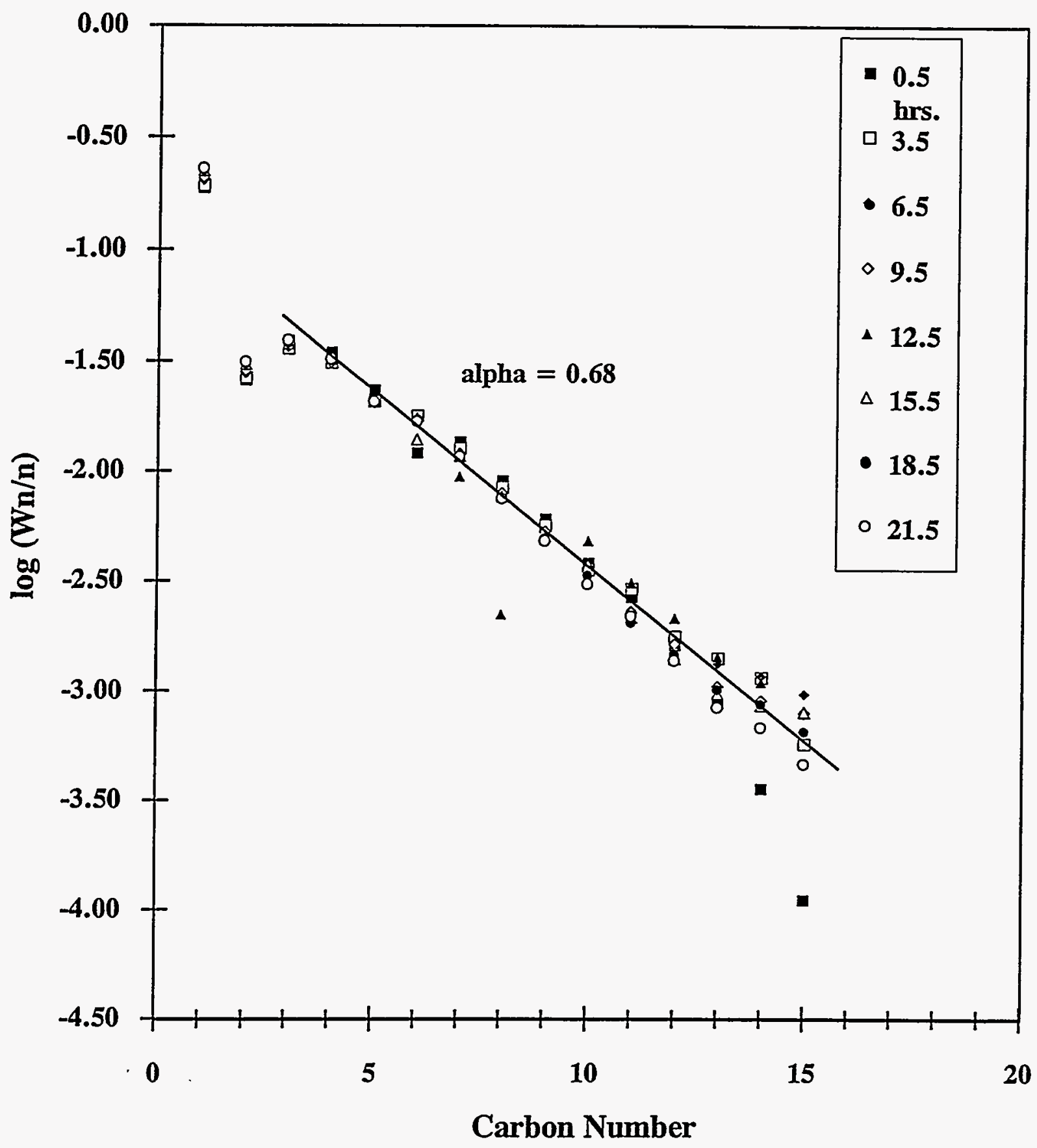




\section{Co.057 - Run \#1}

\begin{tabular}{|c|c|c|c|c|}
\hline Co wt\% & NM & wt \% & Promotor wt\% & Support \\
\hline 20 & $\mathbf{R e}$ & 1.00 & $\mathrm{La2O3} 1.00$ & $\mathrm{SiO2}$ \\
\hline
\end{tabular}

\section{SUMMARY REACTION DATA}

Reaction Conditions:

$$
\begin{array}{ll}
\mathrm{P}=1.0 \mathrm{~atm} & \mathrm{CO} 2(\mathrm{~g} / \mathrm{g} \mathrm{cat} / \mathrm{hr})=0.029 \\
\mathrm{~T}=220^{\circ} \mathrm{C} & \mathrm{CO} 2(\% \text { of } \mathrm{CO})=0.1 \\
\mathrm{H} 2 / \mathrm{CO}=2 & \mathrm{O} / \mathrm{P}=4.65 \\
\text { weight of catalyst }=0.183 \mathrm{~g} & \\
\text { WHSV }=14.031 / \mathrm{hr} & \\
\text { time on stream }=18.5 \mathrm{hrs} &
\end{array}
$$

\begin{tabular}{|l|c|}
\hline CO conversion (\%) & 2.8 \\
\hline rate (g CH2/g cat/hr) & 0.17 \\
\hline alpha & 0.73 \\
\hline $\mathrm{C} 1$ (wt\%) & 23.4 \\
\hline $\mathrm{C} 2-\mathrm{C} 4(\mathrm{wt} \%)$ & 23.2 \\
\hline $\mathrm{C} 5-\mathrm{C} 12(\mathrm{wt} \%)$ & 42.5 \\
\hline $\mathrm{C} 13+(\mathrm{wt} \%)$ & 10.9 \\
\hline
\end{tabular}


Performance of $\mathbf{C o . 0 5 7}$

Dates: 11/27/94 - 11/28/94 Run \#1

flow rate $=90.0 \mathrm{cc} / \mathrm{min}$, loading $=0.2 \mathrm{~g}, \mathrm{WHSV}=14.01 / \mathrm{hr}, \mathrm{H} 2 / \mathrm{CO}$ ratio in feed $=2$

\begin{tabular}{|c|c|c|c|c|c|c|}
\hline time on stream, hr & 0.5 & 3.5 & 6.5 & 9.5 & 12.5 & 15.5 \\
\hline reaction temperature, ${ }^{\circ} \mathrm{C}$ & 220 & 220 & 220 & 220 & 220 & 220 \\
\hline pressure, atm & 1.0 & 1.0 & 1.0 & 1.0 & 1.0 & 1.0 \\
\hline flow, cc/min & 90.0 & 90.0 & 90.0 & 90.0 & 90.0 & 90.0 \\
\hline \multicolumn{7}{|c|}{ C1 - C15 product distribution, weight \% } \\
\hline $\mathrm{C} 1$ & 22.84 & 24.29 & 25.01 & 25.25 & 25.82 & 25.87 \\
\hline $\mathrm{C} 2$ & 4.79 & 4.98 & 5.08 & 5.14 & 5.25 & 5.31 \\
\hline C3 & 10.28 & 10.02 & 9.94 & 9.91 & 10.02 & 10.07 \\
\hline C4 & 11.06 & 10.48 & 10.34 & 10.26 & 10.33 & 10.33 \\
\hline C5 & 11.78 & 11.00 & 10.81 & 10.76 & 10.83 & 10.79 \\
\hline C6 & 9.90 & 9.31 & 9.39 & 9.47 & 8.49 & 8.67 \\
\hline C7 & 8.33 & 7.83 & 7.72 & 7.73 & 7.75 & 7.72 \\
\hline C8 & 6.34 & 5.87 & 5.78 & 5.71 & 5.71 & 5.69 \\
\hline C9 & 4.80 & 4.42 & 4.38 & 4.29 & 4.31 & 4.26 \\
\hline $\mathrm{C} 10$ & 3.61 & 3.39 & 3.31 & 3.27 & 3.35 & 3.19 \\
\hline C11 & 2.47 & 2.39 & 2.30 & 2.34 & 2.26 & 2.21 \\
\hline $\mathrm{C} 12$ & 1.70 & 1.75 & 1.76 & 1.72 & 1.71 & 1.66 \\
\hline $\mathrm{C} 13$ & 1.10 & 1.41 & 1.40 & 1.34 & 1.33 & 1.32 \\
\hline $\mathrm{C} 14$ & 0.74 & 1.49 & 1.43 & 1.38 & 1.33 & 1.37 \\
\hline C15 & 0.26 & 1.37 & 1.36 & 1.43 & 1.49 & 1.54 \\
\hline alpha chain growth probability & 0.62 & 0.72 & 0.72 & 0.73 & 0.73 & 0.73 \\
\hline \multicolumn{7}{|c|}{ C1 - C50 estimated total product distribution, weight $\%$} \\
\hline $\mathrm{C} 1$ & 23.4 & 22.5 & 23.1 & 23.2 & 23.5 & 23.5 \\
\hline $\mathrm{C} 2-\mathrm{C} 4$ & 26.7 & 23.6 & 23.5 & 23.2 & 23.3 & 23.3 \\
\hline $\mathrm{C} 5-\mathrm{C} 12$ & 48.1 & 44.8 & 44.4 & 44.1 & 43.2 & 43.0 \\
\hline $\mathrm{C} 13-\mathrm{C} 50$ & 1.8 & 9.1 & 9.0 & 9.5 & 9.9 & 10.2 \\
\hline $\mathrm{CO}$ conversion, $\%$ & 3.5 & 3.4 & 3.2 & 3.0 & 2.9 & 2.8 \\
\hline rate, $\mathrm{g} \mathrm{CH} 2 / \mathrm{g} \mathrm{cat} / \mathrm{hr}$ & 0.21 & 0.21 & 0.20 & 0.19 & 0.18 & 0.17 \\
\hline $\mathrm{CO} 2$ formation, \% & 0.2 & 0.2 & 0.2 & 0.2 & 0.2 & 0.2 \\
\hline
\end{tabular}




\section{Performance of Co.057}

\section{Dates: 11/27/94 - 11/28/94 Run \#1}

\begin{tabular}{lcc} 
flow rate $=90.0 \mathrm{cc} / \mathrm{min}$, loading $=0.2 \mathrm{~g}$, WHSV $=14.0$ & $1 / \mathrm{hr}, \mathrm{H} 2 / \mathrm{CO}$ ratio in feed $=2$ \\
\hline & 18.5 & 21.5 \\
time on stream, hr & 220 & 220 \\
reaction temperature, ${ }^{\circ} \mathrm{C}$ & 1.0 & 1.0 \\
pressure, atm & 90.0 & 90.0 \\
flow, cc/min & & \\
\hline & & \\
C1 - C15 product distribution, weight \% & & \\
& & \\
C1 & 26.13 & 22.25 \\
& & \\
C2 & 5.41 & 4.62 \\
C3 & 10.22 & 8.64 \\
C4 & 10.28 & 8.73 \\
& & \\
C5 & 10.85 & 13.01 \\
C6 & 8.54 & 7.37 \\
C7 & 7.59 & 9.25 \\
C8 & 5.66 & 6.91 \\
C9 & 4.15 & 5.10 \\
C10 & 3.12 & 4.03 \\
C11 & 2.13 & 2.67 \\
C12 & 1.59 & 2.01 \\
C13 & 1.29 & 1.61 \\
C14 & 1.39 & 1.62 \\
C15 & 1.66 & 2.19 \\
& & \\
alpha & 0.73 & 0.75 \\
& & \\
\hline
\end{tabular}

C1 - C50 estimated total product distribution, weight \%

$\begin{array}{lll}\text { C1 } & 23.4 & 19.2 \\ \text { C2 - C4 } & 23.2 & 19.0 \\ \text { C5 - C12 } & 42.5 & 47.3 \\ \text { C13 - C50 } & 10.9 & 14.5\end{array}$

CO conversion, \%

rate, $\mathrm{g} \mathrm{CH} 2 / \mathrm{g} \mathrm{cat} / \mathrm{hr}$

$\mathrm{CO} 2$ formation, \%
$2.8 \quad 3.3$

$0.17 \quad 0.20$

$0.1 \quad 0.2$ 
Time-on-Stream Plot for Co.057 - Run \#1

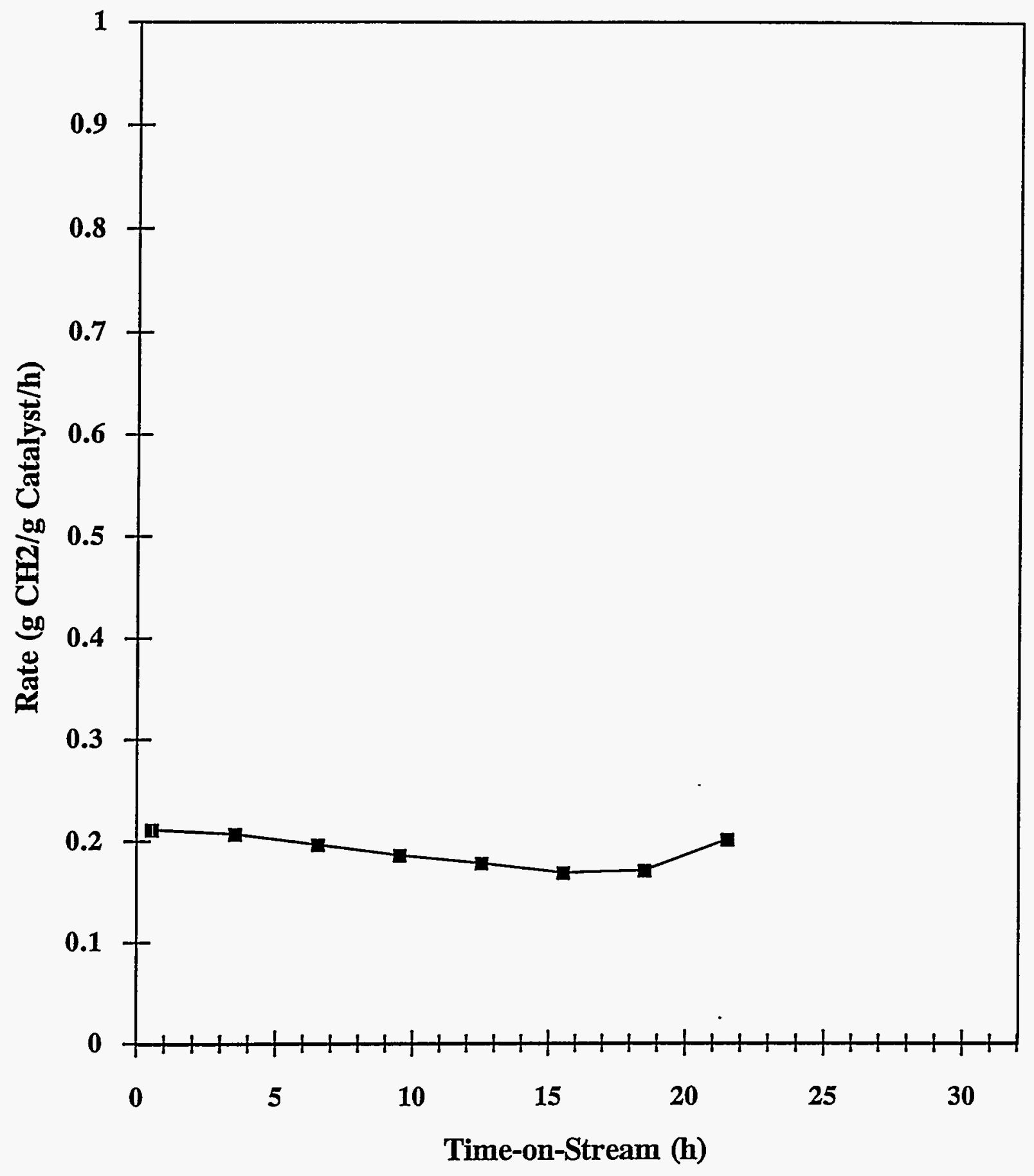


Schulz-Flory Plot for Co.057 - Run \#1

Time on Stream (hrs)

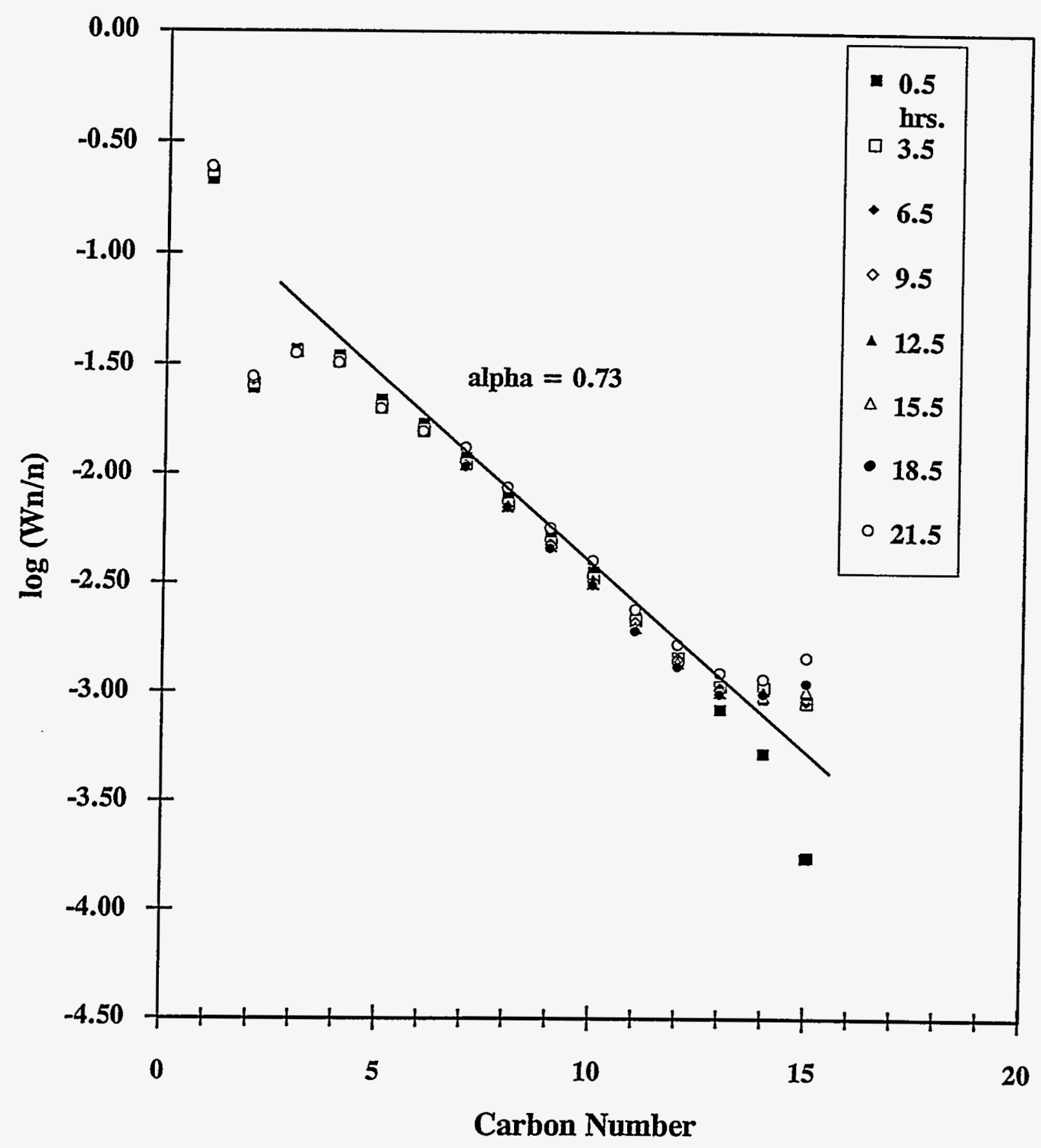




\section{Co.060 - Run \#1}

\begin{tabular}{|c|c|c|c|}
\hline Co wt\% & NM wt \% & Promotor wt\% & Support \\
\hline 30 & & & $\mathrm{Al2O3}$ \\
\hline
\end{tabular}

\section{SUMMARY REACTION DATA}

Reaction Conditions:

$$
\begin{array}{ll}
\mathrm{P}=1.0 \mathrm{~atm} & \mathrm{CO} 2(\mathrm{~g} / \mathrm{g} \mathrm{cat} / \mathrm{hr})=0.023 \\
\mathrm{~T}=220^{\circ} \mathrm{C} & \mathrm{CO} 2(\% \text { of } \mathrm{CO})=0.1 \\
\mathrm{H} 2 / \mathrm{CO}=2 & \mathrm{O} / \mathrm{P}=3.82 \\
\text { weight of catalyst }=0.176 \mathrm{~g} & \\
\text { WHSV }=14.591 / \mathrm{hr} & \\
\text { time on stream }=24.5 \mathrm{hrs} &
\end{array}
$$

\begin{tabular}{|l|c|}
\hline \hline CO conversion (\%) & 3.8 \\
\hline rate (g CH2/g cat/hr) & 0.24 \\
\hline alpha & 0.69 \\
\hline $\mathrm{C} 1$ (wt\%) & 25.0 \\
\hline $\mathrm{C} 2-\mathrm{C} 4(w \mathrm{t} \%)$ & 26.2 \\
\hline $\mathrm{C} 5-\mathrm{C} 12(\mathrm{wt} \%)$ & 43.0 \\
\hline $\mathrm{C} 13+(w \mathrm{t} \%)$ & 5.8 \\
\hline
\end{tabular}




\section{Performance of $\mathbf{C o . 0 6 0}$ \\ Dates: 11/21/94 - 11/22/94 Run\#1}

flow rate $=90.0 \mathrm{cc} / \mathrm{min}$, loading $=0.2 \mathrm{~g}, \mathrm{WHSV}=14.61 / \mathrm{hr}, \mathrm{H} 2 / \mathrm{CO}$ ratio in feed $=2$

\begin{tabular}{|c|c|c|c|c|c|c|}
\hline time on stream, $\mathrm{hr}$ & 0.5 & 3.5 & 6.5 & 9.5 & 12.5 & 15.5 \\
\hline reaction temperature, ${ }^{\circ} \mathrm{C}$ & 220 & 220 & 220 & 220 & 220 & 220 \\
\hline pressure, atm & 1.0 & 1.0 & 1.0 & 1.0 & 1.0 & 1.0 \\
\hline flow, $\mathrm{cc} / \mathrm{min}$ & 90.0 & 90.0 & 90.0 & 90.0 & 90.0 & 90.0 \\
\hline \multicolumn{7}{|c|}{ C1 - C15 product distribution, weight \% } \\
\hline $\mathrm{Cl}$ & 22.80 & 23.87 & 24.74 & 25.26 & 24.94 & 25.45 \\
\hline $\mathrm{C} 2$ & 4.44 & 4.55 & 4.65 & 4.73 & 4.66 & 4.72 \\
\hline $\mathrm{C} 3$ & 11.31 & 11.25 & 11.36 & 11.38 & 11.12 & 11.22 \\
\hline $\mathrm{C} 4$ & 11.70 & 11.48 & 11.49 & 11.34 & 11.20 & 11.25 \\
\hline C5 & 11.56 & 11.38 & 11.29 & 11.42 & 11.15 & 11.17 \\
\hline C6 & 9.19 & 9.02 & 8.77 & 9.22 & 8.98 & 9.00 \\
\hline $\mathrm{C} 7$ & 7.89 & 7.78 & 7.66 & 7.24 & 7.55 & 7.45 \\
\hline C8 & 5.83 & 5.71 & 5.57 & 5.33 & 5.55 & 5.44 \\
\hline $\mathrm{Cg}$ & 4.40 & 4.25 & 4.13 & 4.02 & 4.16 & 4.03 \\
\hline $\mathrm{C} 10$ & 3.38 & 3.18 & 3.11 & 3.03 & 3.12 & 3.04 \\
\hline C11 & 2.51 & 2.33 & 2.23 & 2.21 & 2.53 & 2.22 \\
\hline $\mathrm{C} 12$ & 1.92 & 1.80 & 1.64 & 1.62 & 1.63 & 1.70 \\
\hline $\mathrm{C} 13$ & 1.33 & 1.33 & 1.28 & 1.21 & 1.31 & 1.21 \\
\hline C14 & 0.98 & 1.11 & 1.14 & 1.05 & 1.15 & 1.12 \\
\hline $\mathrm{C} 15$ & 0.76 & 0.96 & 0.94 & 0.94 & 0.97 & 0.97 \\
\hline alpha chain growth probability & 0.68 & 0.69 & 0.69 & 0.69 & 0.70 & 0.70 \\
\hline \multicolumn{7}{|c|}{$\mathrm{C} 1$ - C50 estimated total product distribution, weight $\%$} \\
\hline $\mathrm{Cl}$ & 22.3 & 22.9 & 23.7 & 24.1 & 23.9 & 24.3 \\
\hline $\mathrm{C} 2-\mathrm{C} 4$ & 26.9 & 26.1 & 26.3 & 26.2 & 25.8 & 26.0 \\
\hline $\mathrm{C} 5-\mathrm{C} 12$ & 45.8 & 44.7 & 43.8 & 43.5 & 43.9 & 43.3 \\
\hline $\mathrm{C} 13-\mathrm{C} 50$ & 5.1 & 6.3 & 6.2 & 6.2 & 6.4 & 6.4 \\
\hline CO conversion, $\%$ & 4.5 & 4.4 & 4.3 & 4.1 & 4.1 & 4.0 \\
\hline rate, $\mathrm{g} \mathrm{CH} 2 / \mathrm{g} \mathrm{cat} / \mathrm{hr}$ & 0.29 & 0.28 & 0.27 & 0.26 & 0.26 & 0.26 \\
\hline $\mathrm{CO} 2$ formation, $\%$ & 0.1 & 0.1 & 0.1 & 0.1 & 0.1 & 0.1 \\
\hline
\end{tabular}




\section{Performance of Co.060}

\section{Dates: 11/21/94 - 11/22/94 Run \#1}

flow rate $=90.0 \mathrm{cc} / \mathrm{min}$, loading $=0.2 \mathrm{~g}, \mathrm{WHSV}=14.61 / \mathrm{hr}, \mathrm{H} 2 / \mathrm{CO}$ ratio in feed $=2$

\begin{tabular}{|c|c|c|c|}
\hline time on stream, hr & 18.5 & 21.5 & 24.5 \\
\hline reaction temperature, ${ }^{\circ} \mathrm{C}$ & 220 & 220 & 220 \\
\hline pressure, atm & 1.0 & 1.0 & 1.0 \\
\hline flow, cc/min & 90.0 & 90.0 & 90.0 \\
\hline \multicolumn{4}{|c|}{ C1 - C15 product distribution, weight \% } \\
\hline $\mathrm{Cl}$ & 25.75 & 25.84 & 26.03 \\
\hline $\mathrm{C} 2$ & 4.76 & 4.77 & 4.80 \\
\hline C3 & 11.26 & 11.23 & 11.26 \\
\hline $\mathrm{C} 4$ & 11.20 & 11.19 & 11.16 \\
\hline C5 & 11.09 & 11.12 & 11.06 \\
\hline C6 & 8.89 & 8.91 & 8.93 \\
\hline C7 & 7.47 & 7.50 & 7.40 \\
\hline $\mathrm{C} 8$ & 5.45 & 5.49 & 5.40 \\
\hline C9 & 4.05 & 4.02 & 3.94 \\
\hline $\mathrm{C} 10$ & 3.06 & 3.06 & 3.01 \\
\hline $\mathrm{C} 11$ & 2.20 & 2.15 & 2.47 \\
\hline $\mathrm{C} 12$ & 1.55 & 1.53 & 1.54 \\
\hline C13 & 1.22 & 1.20 & 1.13 \\
\hline $\mathrm{C} 14$ & 1.08 & 1.05 & 0.99 \\
\hline C15 & 0.96 & 0.94 & 0.87 \\
\hline alpha chain growth probability & 0.70 & 0.69 & 0.69 \\
\hline \multicolumn{4}{|c|}{$\mathrm{C} 1$ - C50 estimated total product distribution, weight \% } \\
\hline $\mathrm{C} 1$ & 24.5 & 24.6 & 25.0 \\
\hline $\mathrm{C} 2-\mathrm{C} 4$ & 25.9 & 25.9 & 26.2 \\
\hline $\mathrm{C} 5-\mathrm{C} 12$ & 43.2 & 43.2 & 43.0 \\
\hline $\mathrm{C} 13-\mathrm{C} 50$ & 6.3 & 6.2 & 5.8 \\
\hline $\mathrm{CO}$ conversion, $\%$ & 4.0 & 3.9 & 3.8 \\
\hline rate, $\mathrm{g} \mathrm{CH} 2 / \mathrm{g}$ cat $/ \mathrm{hr}$ & 0.25 & 0.25 & 0.24 \\
\hline $\mathrm{CO} 2$ formation, $\%$ & 0.1 & 0.1 & 0.1 \\
\hline
\end{tabular}


Time-on-Stream Plot for Co.060 - Run \#1

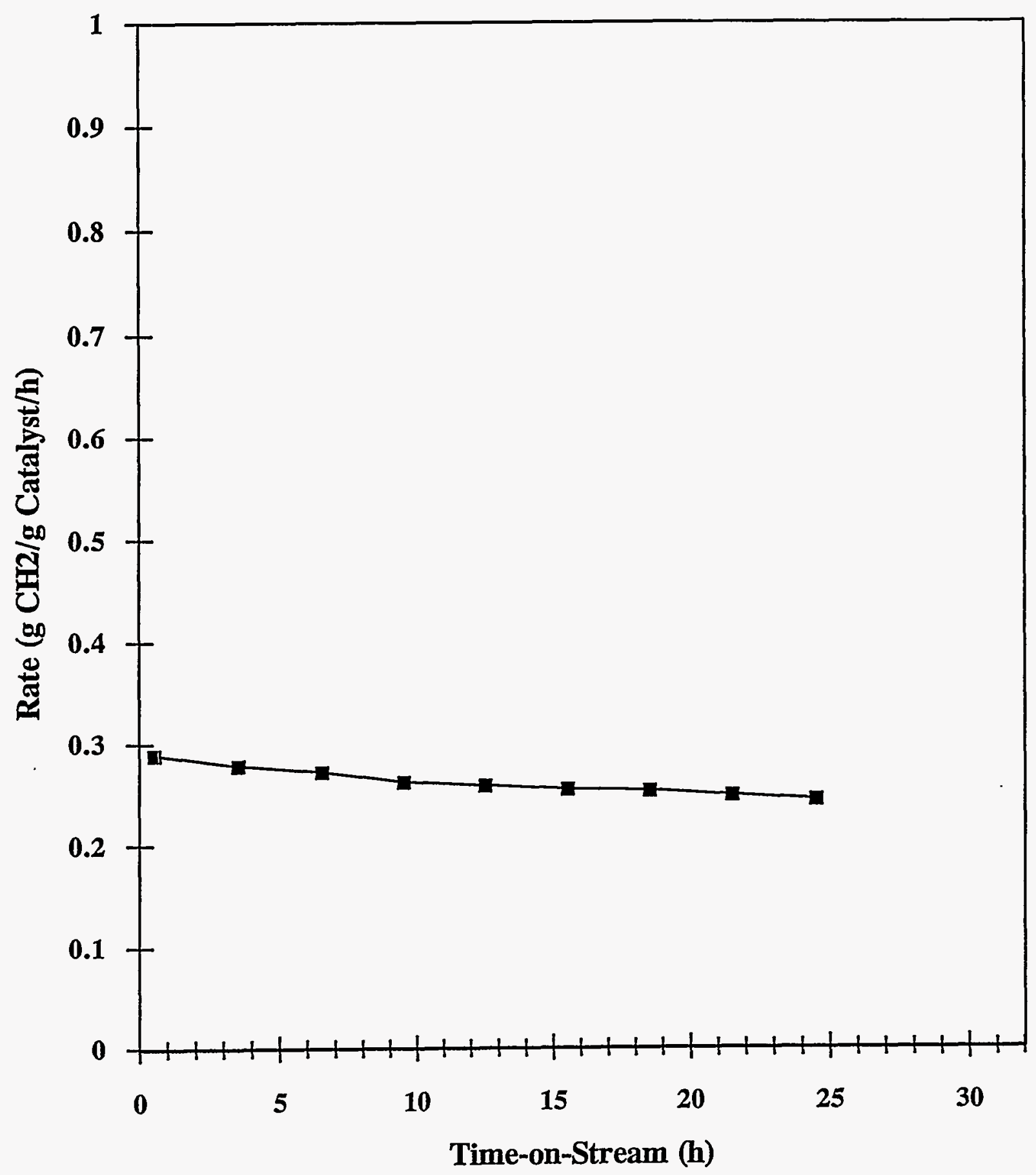


Schulz-Flory Plot for Co.060 - Run \#1

Time on Stream (hrs)

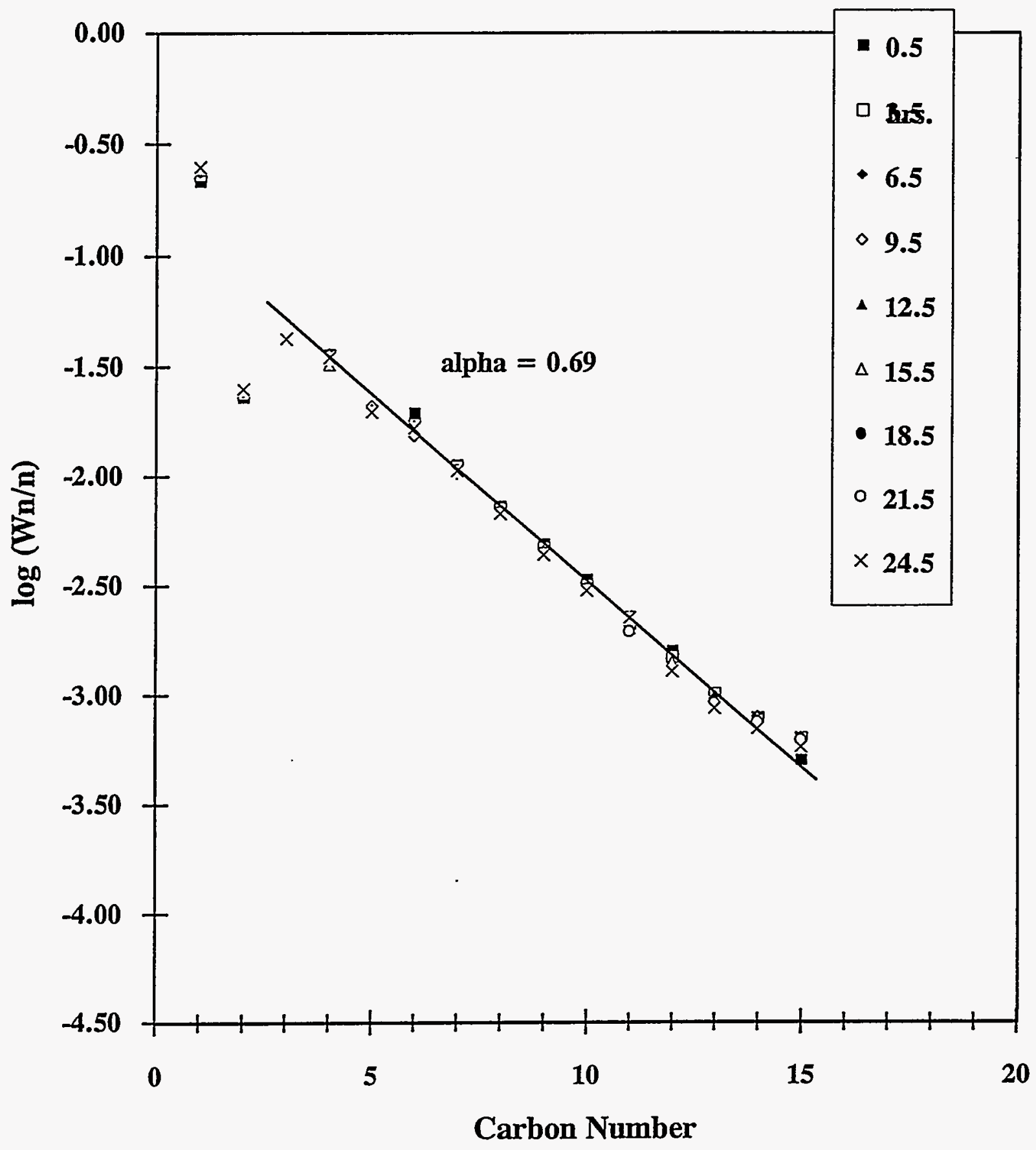




\section{Cal.10 - Run \#1}

\begin{tabular}{|c|c|c|c|c|}
\hline Co wt\% & NM & wt \% & Promo & Support \\
\hline 20 & $\mathbf{R u}$ & 0.50 & K $\quad 0.30$ & $\mathrm{Al} 2 \mathrm{O3}$ \\
\hline
\end{tabular}

\section{SUMMARY REACTION DATA}

Reaction Conditions:

$$
\begin{array}{ll}
\mathrm{P}=1.0 \mathrm{~atm} & \mathrm{CO} 2(\mathrm{~g} / \mathrm{g} \mathrm{cat} / \mathrm{hr})=0.045 \\
\mathrm{~T}=220^{\circ} \mathrm{C} & \mathrm{CO} 2(\% \text { of } \mathrm{CO})=0.2 \\
\mathrm{H} 2 / \mathrm{CO}=2 & \mathrm{O} / \mathrm{P}=1.75 \\
\text { weight of catalyst }=0.198 \mathrm{~g} & \\
\text { WHSV }=12.991 / \mathrm{hr} & \\
\text { time on stream }=24.5 \mathrm{hrs} &
\end{array}
$$

\begin{tabular}{||l|c|}
\hline CO conversion (\%) & 3.5 \\
\hline rate (g CH2/g cat/hr) & 0.20 \\
\hline alpha & 0.74 \\
\hline $\mathrm{C} 1$ (wt\%) & 23.5 \\
\hline $\mathrm{C} 2-\mathrm{C} 4$ (wt\%) & 19.9 \\
\hline $\mathrm{C} 5-\mathrm{C} 12(\mathrm{wt} \%)$ & 46.0 \\
\hline $\mathrm{C} 13+(w t \%)$ & 10.6 \\
\hline
\end{tabular}




\section{Performance of Cal.10 \\ Dates: 11/18/94 - 11/19/94 Run \#1}

flow rate $=90.0 \mathrm{cc} / \mathrm{min}$, loading $=0.2 \mathrm{~g}, \mathrm{WHSV}=13.01 / \mathrm{hr}, \mathrm{H} 2 / \mathrm{CO}$ ratio in feed $=2$

\begin{tabular}{|c|c|c|c|c|c|c|}
\hline time on stream, hr & 0.5 & 3.5 & 6.5 & 9.5 & 12.5 & 15.5 \\
\hline reaction temperature, ${ }^{\circ} \mathrm{C}$ & 220 & 220 & 220 & 220 & 220 & 220 \\
\hline pressure, atm & 1.0 & 1.0 & 1.0 & 1.0 & 1.0 & 1.0 \\
\hline flow, cc/min & 90.0 & 90.0 & 90.0 & 90.0 & 90.0 & 90.0 \\
\hline \multicolumn{7}{|c|}{ C1 - C15 product distribution, weight \% } \\
\hline $\mathrm{Cl}$ & 22.57 & 24.99 & 25.69 & 25.46 & 24.87 & 24.25 \\
\hline $\mathrm{C} 2$ & 4.04 & 4.30 & 4.35 & 4.33 & 4.26 & 4.20 \\
\hline C3 & 8.21 & 8.19 & 8.20 & 8.21 & 8.19 & 8.10 \\
\hline $\mathrm{C} 4$ & 9.30 & 8.92 & 8.67 & 8.75 & 8.78 & 8.62 \\
\hline C5 & 10.14 & 9.26 & 9.01 & 9.10 & 9.14 & 9.11 \\
\hline C6 & 9.01 & 7.65 & 8.23 & 8.20 & 8.37 & 8.31 \\
\hline C7 & 9.16 & 8.23 & 7.89 & 8.01 & 8.12 & 8.20 \\
\hline $\mathrm{C} 8$ & 7.48 & 6.66 & 6.34 & 6.51 & 6.62 & 6.73 \\
\hline $\mathrm{Cg}$ & 6.04 & 5.22 & 5.14 & 5.25 & 5.34 & 5.52 \\
\hline C10 & 4.98 & 4.78 & 4.27 & 4.36 & 4.48 & 4.59 \\
\hline C11 & 3.85 & 3.55 & 3.32 & 3.51 & 3.58 & 3.73 \\
\hline C12 & 2.78 & 2.86 & 2.81 & 2.85 & 2.76 & 2.92 \\
\hline C13 & 1.61 & 2.51 & 2.26 & 2.13 & 2.11 & 2.25 \\
\hline C14 & 0.63 & 2.25 & 2.01 & 1.77 & 1.78 & 1.87 \\
\hline C15 & 0.21 & 0.63 & 1.83 & 1.57 & 1.60 & 1.61 \\
\hline alpha chain growth probability & 0.61 & 0.68 & 0.75 & 0.74 & 0.74 & 0.74 \\
\hline \multicolumn{7}{|c|}{ C1 - C50 estimated total product distribution, weight $\%$} \\
\hline $\mathrm{C} 1$ & 24.0 & 26.0 & 23.5 & 23.8 & 23.2 & 22.7 \\
\hline $\mathrm{C} 2-\mathrm{C} 4$ & 22.9 & 22.2 & 19.4 & 19.9 & 19.8 & 19.6 \\
\hline $\mathrm{C} 5-\mathrm{C} 12$ & 51.6 & 47.3 & 44.4 & 45.3 & 45.8 & 46.3 \\
\hline $\mathrm{C} 13-\mathrm{C50}$ & 1.5 & 4.5 & 12.7 & 11.0 & 11.1 & 11.3 \\
\hline CO conversion, \% & 3.6 & 3.8 & 4.3 & 3.9 & 3.8 & 3.7 \\
\hline rate, g CH2/g cat $/ \mathrm{hr}$ & 0.21 & 0.21 & 0.24 & 0.22 & 0.22 & 0.21 \\
\hline $\mathrm{CO} 2$ formation, $\%$ & 0.3 & 0.5 & 0.3 & 0.2 & 0.2 & 0.2 \\
\hline
\end{tabular}




\section{Performance of Cal.10}

\section{Dates: 11/18/94 - 11/19/94 Run \#1}

flow rate $=90.0 \mathrm{cc} / \mathrm{min}$, loading $=0.2 \mathrm{~g}, \mathrm{WHSV}=13.0 \mathrm{1} / \mathrm{hr}, \mathrm{H} 2 / \mathrm{CO}$ ratio in feed $=2$

\begin{tabular}{lccc}
\hline & 18.5 & 21.5 & 24.5 \\
time on stream, $\mathrm{hr}$ & 220 & 220 & 220 \\
reaction temperature, ${ }^{\circ} \mathrm{C}$ & 1.0 & 1.0 & 1.0 \\
pressure, atm & 90.0 & 90.0 & 90.0 \\
\hline
\end{tabular}

C1 - C15 product distribution, weight \%

C1

C2

C3

$\mathrm{C} 4$

C5

C6

C7

C8

C9

C10

C11

C12

C13

C14

C15

alpha chain growth probability

\section{$23.56 \quad 23.37 \quad 24.84$}

$\begin{array}{lll}4.14 & 4.13 \quad 4.29\end{array}$

$\begin{array}{lll}8.03 & 8.02 & 8.24\end{array}$

$\begin{array}{lll}8.58 & 8.55 \quad 8.57\end{array}$

$\begin{array}{lll}9.12 & 9.14 & 9.10\end{array}$

$\begin{array}{lll}8.33 & 8.47 & 8.33\end{array}$

$\begin{array}{lll}8.28 & 8.33 \quad 8.10\end{array}$

$\begin{array}{lll}6.81 & 6.79 & 6.61\end{array}$

$\begin{array}{lll}5.61 & 5.68 & 5.39\end{array}$

$\begin{array}{lll}4.71 & 4.47 \quad 4.53\end{array}$

$\begin{array}{lll}3.75 & 4.01 & 3.60\end{array}$

$\begin{array}{lll}3.05 & 2.94 & 2.85\end{array}$

$\begin{array}{lll}2.45 & 2.39 & 2.22\end{array}$

$\begin{array}{lll}2.03 & 2.01 & 1.81\end{array}$

$\begin{array}{lll}1.55 & 1.68 & 1.51\end{array}$

$\begin{array}{lll}0.74 & 0.75 & 0.74\end{array}$

$\mathrm{C} 1$ - C50 estimated total product distribution, weight \%
C1
$\mathrm{C} 2-\mathrm{C} 4$
$22.3 \quad 21.9 \quad 23.5$
$\mathrm{C} 5-\mathrm{C} 12$
$\begin{array}{lll}19.7 & 19.4 & 19.9\end{array}$
C13 - C50
$\begin{array}{lll}47.0 & 46.8 & 46.0\end{array}$
$\begin{array}{lll}11.0 & 11.9 & 10.6\end{array}$

CO conversion, \%

rate, $\mathrm{g} \mathrm{CH} 2 / \mathrm{g} \mathrm{cat} / \mathrm{hr}$

$\mathrm{CO} 2$ formation, \% $\begin{array}{lll}3.4 & 3.4 & 3.5\end{array}$

$\begin{array}{lll}0.20 & 0.19 & 0.20\end{array}$

$\begin{array}{lll}0.2 & 0.2 & 0.2\end{array}$ 
Time-on-Stream Plot for Cal.10 - Run \#1

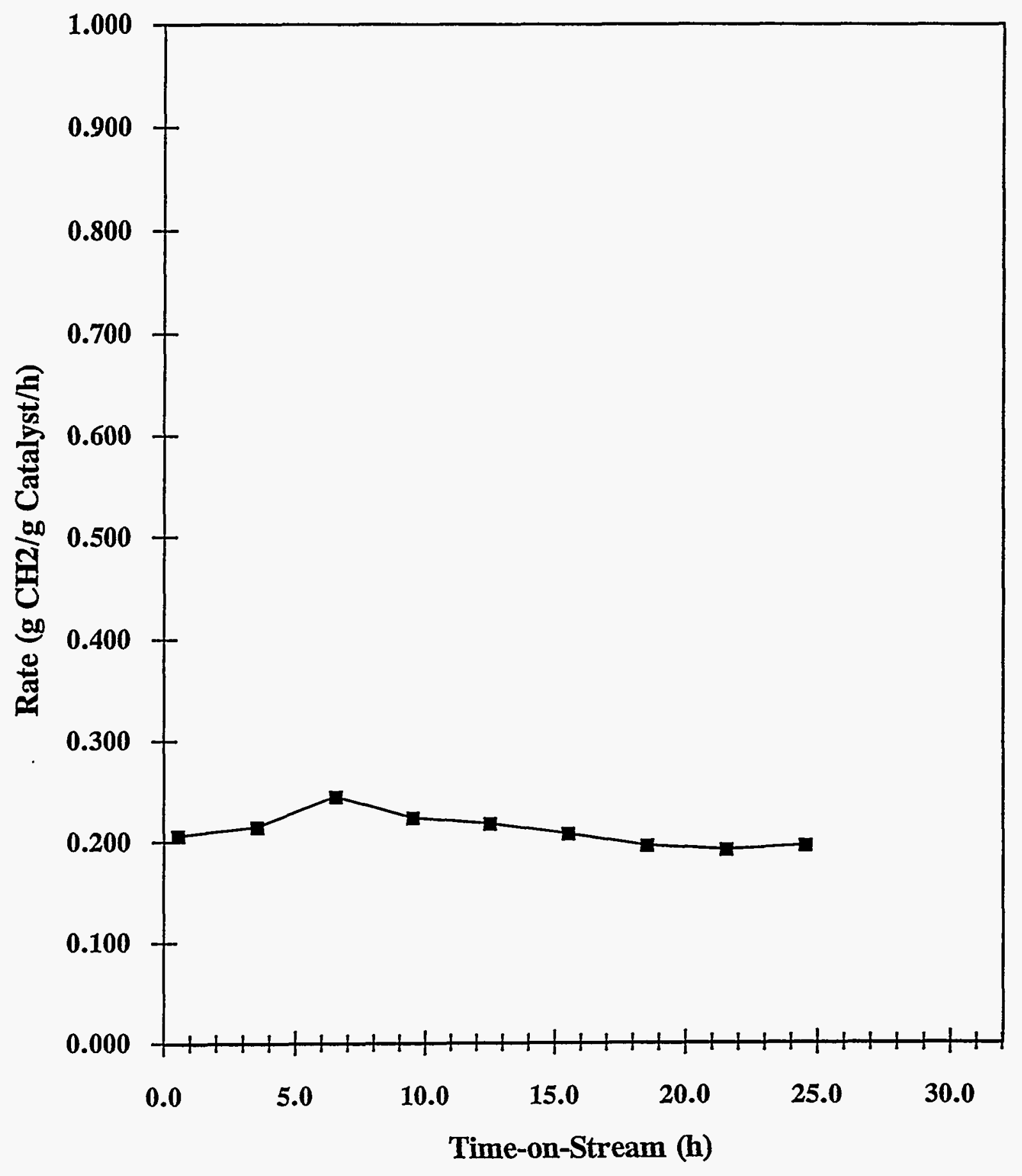


Schulz-Flory Plot for Cal.10 - Run \#1

Time on Stream (hrs)

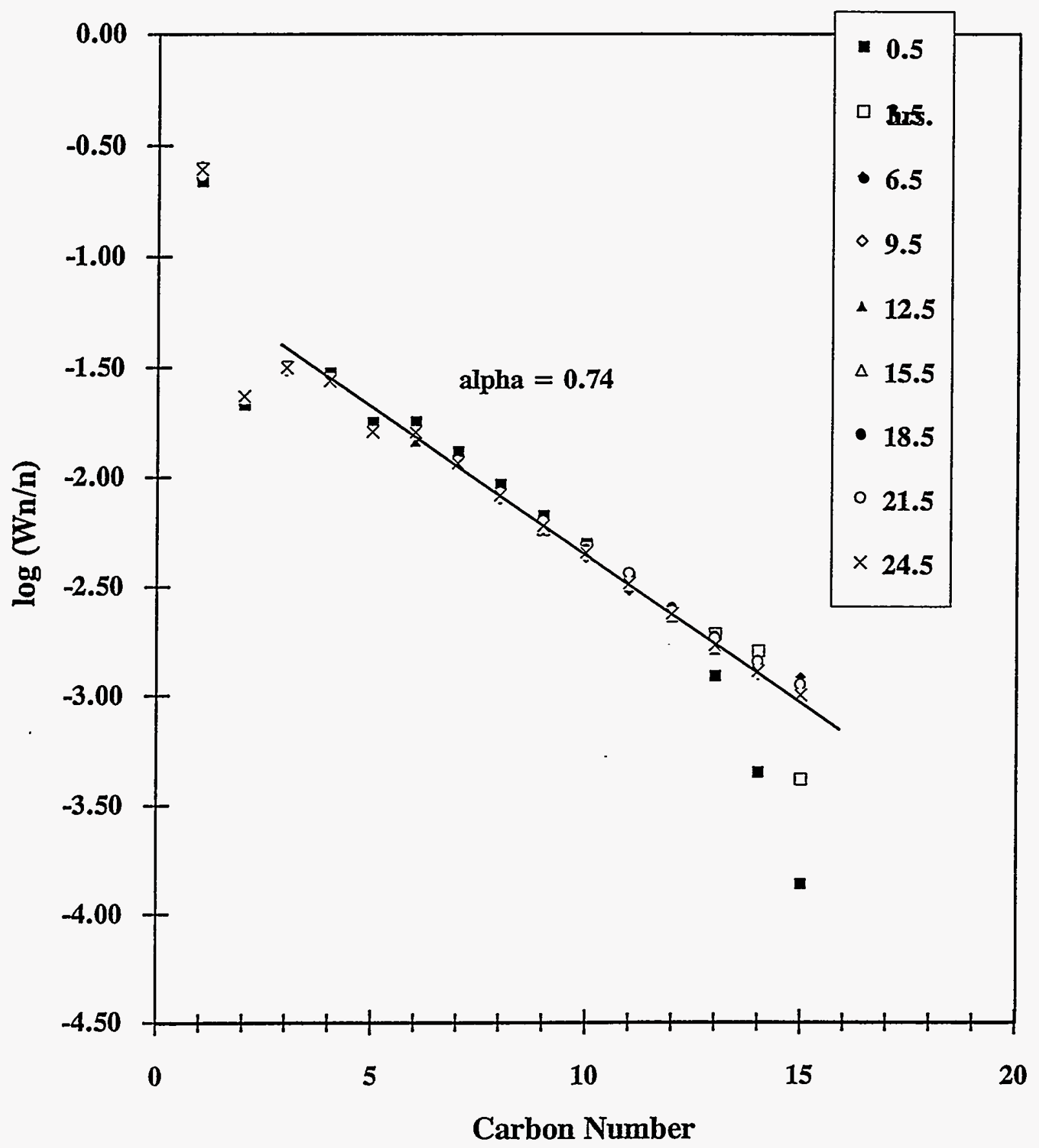




\section{APPENDIX C}

Chronolgy of Runs in Slurry Bubble Column Reactors 


\section{APPENDIX C}

\section{CHRONOLOGY OF RUNS MADE IN THE SLURRY BUBBLE COLUMN REACTORS DURING THE FOURTH QUARTER OF 1994}

\section{Task 2.0 - Catalyst Testing}

\section{Subtask 2.2 -- "Slurry Bubble Column Testing"}

Run No. 22 in the M4-SBCR was started on October 3rd with a blend of two catalysts: 15.5 gm of Catalyst No. WGS.09 and 15.0 gm of Catalyst No. Co.005. The first catalyst is a water-gas shift catalyst and the second is a methane reduction catalyst. The $\mathrm{CO}$ conversion with this blend at startup conditions was considerably higher than observed with Catalyst No. Co.005 in Run 15 in M3SBCR, $35.6 \%$ vs. $27.1 \%$. Also, the $\mathrm{CO}_{2}$ selectivity was much higher, $6.3 \%$ vs. $0.8 \%$. Some watergas shift activity has occurred with this catalyst blend as demonstrated by the higher $\mathrm{CO}_{2}$ yield and increase in $\mathrm{CO}$ conversion. Several run periods were made in which the $\mathrm{H}_{2} / \mathrm{CO}$ ratio was lowered to $1 / 1$ and $0.7 / 1$ at $240^{\circ}, 260^{\circ}$, and $280^{\circ} \mathrm{C}$ reaction temperatures. The $\mathrm{CO}$ conversion decreased as the $\mathrm{H}_{2} / \mathrm{CO}$ ratio was decreased and the $\mathrm{CO}$ conversion increased when the reaction temperature was raised. Water-gas shift activity was observed at all run conditions.

Run No. 30 in the M3-SBCR was started on October 10th with a charge of $29.6 \mathrm{gm}$ of Catalyst No. Co.053-2. The purpose of this run was to run at conditions that would yield the highest CO conversion attainable in the SBCR. The CO conversion at startup conditions was $41.8 \%$, the same as observed previously in Run 29 with the same catalyst charge. The $\mathrm{CO}$ conversion increased to $46.9 \%$ at $260^{\circ} \mathrm{C}$ reaction temperature. The $\mathrm{CO}$ conversion, however, dropped to $41.4 \%$ when the reaction temperature was increased to $280^{\circ} \mathrm{C}$. The reaction temperature was increased to $300^{\circ} \mathrm{C}$, $310^{\circ} \mathrm{C}$, and finally to $320^{\circ} \mathrm{C}$, but the $\mathrm{CO}$ conversion did not exceed $40 \%$ at any of these temperatures. The run was shut down after 279 hours after the gas preheater plugged when the heater temperature reached $550^{\circ} \mathrm{C}$. It is speculated that the catalyst activity began to decrease above $260^{\circ} \mathrm{C}$ because the reaction temperature may have been increased too rapidly. This run will be repeated using the M4SBCR.

Run No. 23 in the M4-SBCR was started on October 17th with a charge of 29.1 gm of Catalyst No. CAL.09. This was a catalyst that was supplied by Calsicat that was hydrogen reduced and coated with Soya. The catalyst was mixed with synfluid, heated, and charged to the SBCR under a nitrogen blanket. The $\mathrm{CO}$ conversion at the initial startup conditions was only $17.4 \%$. The run was terminated after two run periods because the catalyst activity was only $60 \%$ of the expected activity.

Run No. 24 in the M4-SBCR was started on October 24th with a charge of $28.5 \mathrm{gm}$ of Catalyst No. Co.053-3. The purpose of this run was to attain the highest $\mathrm{CO}$ conversion possible. The CO conversion at startup conditions was $50.4 \%$, which is the highest conversion observed to date. The M4-SBCR appears to yield a little higher catalyst activity than M3-SBCR for some unknown reason. The $\mathrm{CO}$ conversion rose to $53.5 \%$ at $250^{\circ} \mathrm{C}$ reaction temperature (Period 2) and 
to $55.3 \%$ at $260^{\circ} \mathrm{C}$ (Period 3). In Period No. 4, the $\mathrm{N}_{2}$ feed rate was decreased from $60 \%$ of the total to $40 \%$ with a corresponding increase in the $\mathrm{H}_{2}$ and $\mathrm{CO}$ feed rates. The $\mathrm{CO}$ conversion dropped to $52.8 \%$, but the production rate increased from 1.36 to $2.13 \mathrm{gm} \mathrm{C}_{1}^{+} / \mathrm{gm}$ catalyst $/ \mathrm{hr}$. The total gas feed rate was then lowered from 900 standard liter per hour (SLH) to 700 SLH for Period 5. The CO conversion rose to $58.9 \%$. Increasing the total feed gas rate to $1080 \mathrm{SLH}\left(50 \% \mathrm{~N}_{2}\right)$ lowered the CO conversion to $42.6 \%$ for Period 6 . At this time the gas feed rates were returned to startup rates for an activity check. The $\mathrm{CO}$ conversion averaged $51.5 \%$ compared to $55.3 \%$ obtained in Period 3 made at the same run conditions. This was considered to be a normal loss of catalyst activity.

For Period 8, the total feed gas rate was reduced from 900 SLH to 632 SLH with about $32 \%$ $\mathrm{N}_{2}$ in the feed. The $\mathrm{CO}$ conversion rose to $58.1 \%$. Lowering the total feed gas rate to $540 \mathrm{SLH}$ $\left(20 \% \mathrm{~N}_{2}\right.$ ) for Period 9 yielded a CO conversion of $60.0 \%$. It was decided to raise the reactor temperature to $270^{\circ} \mathrm{C}$ to further increase the $\mathrm{CO}$ conversion. The reaction temperature; however, increased rapidly and could not be controlled because the SBCR has no provision for heat removal. The reactor stabilized at $275^{\circ} \mathrm{C}$ for about 5 hours with a CO conversion of $72 \%$. The reactor temperature then rose to $292^{\circ} \mathrm{C}$ before dropping to $255^{\circ} \mathrm{C}$ by the next morning. The reactor was stabilized at $260^{\circ} \mathrm{C}$ for an activity check. The CO conversion was only $49 \%$, so it was decided to terminate the run. A chilled water cooling coil will be installed in the feed gas line before attempting high $\mathrm{CO}$ conversion runs. This run demonstrated that higher $\mathrm{CO}$ conversion levels can be obtained by reducing the $\mathrm{N}_{2}$ feed composition and total gas feed rate. Also, a method to remove the heat of reaction must be found for the SBCR before attempting to reach higher $\mathrm{CO}$ conversion levels.

Run No. 31 in the M3-SBCR was started on October 31st with a charge of $15.0 \mathrm{gm}$ of Catalyst No. Co.055. This catalyst contained $20 \mathrm{wt} \%$ Co plus $1.0 \mathrm{wt} \%$ Re and $1.0 \mathrm{wt} \% \mathrm{La}_{2} \mathrm{O}_{3}$ promoters on an alumina catalyst support. The $\mathrm{CO}$ conversion at startup conditions was good, $30.0 \%$, with a high production rate, $1.46 \mathrm{gm} \mathrm{C}_{1}^{+} / \mathrm{gm}$ catalyst/hr, but the $\mathrm{CH}_{4}$ selectivity, however, was quite high, $23.7 \%$. The catalyst is similar to Co.001, the original startup benchmark catalyst used in Run 2 in the M3-SBCR, but without $\mathrm{K}$ added.

Run No. 25 in the M4-SBCR was started on November 8th with a charge of $15.0 \mathrm{gm}$ of Catalyst No. Co.056. This catalyst contained $20 \mathrm{wt} \%$ Co plus $8.5 \% \mathrm{La}_{2} \mathrm{O}_{3}$ on silica catalyst support. The $\mathrm{CO}$ conversion at startup conditions was only $11.4 \%$ and $4.5 \%$ at $220^{\circ} \mathrm{C}$ reaction temperature. The run was terminated after 63 hours on stream due to the low catalyst activity.

Run No. 32 in the M3-SBCR was started on November 14th with a charge of $16.4 \mathrm{gm}$ of Catalyst No. Co. 044 . This catalyst contained $20 \mathrm{wt} \%$ Co plus $15 \% \mathrm{Zr}$ promoter on silica support. The purpose of this run was to determine the effect of adding a larger amount of $\mathrm{Zr}$ on catalyst activity. The greater amount of $Z r$ yielded a $21 \% \mathrm{CO}$ conversion compared to $25 \% \mathrm{CO}$ conversion with an $8.5 \% \mathrm{Zr}$ on a cobalt-silica catalyst and $23.2 \% \mathrm{CO}$ conversion with a $0.7 \% \mathrm{Zr}$ promoter on a cobalt-silica catalyst.

Run No. 26 in the M4-SBCR was started on November 17th with a charge of $15.6 \mathrm{gm}$ of Catalyst No. CAL.10. This catalyst which was received from Calsicat contained $20 \mathrm{wt} \%$ Co with $0.3 \% \mathrm{~K}$ and $0.5 \% \mathrm{Ru}$ on Condea alumina. The Condea alumina is similar to Vista B alumina which has demonstrated good attrition resistance. The CO conversion at startup conditions was only $21.9 \%$ 
versus $26.5 \%$ obtained with Catalysts CAL. 04 and CAL. 05 which were made with Vista B alumina support. It was decided not to follow the usual schedule of run conditions, but to investigate the effect of lowering the total syngas and nitrogen flows into the reactor on catalyst activity. The reaction was held at $240^{\circ} \mathrm{C}, 450$ psi pressure, and $2 / 1 \mathrm{H}_{2} / \mathrm{CO}$ feed ratio with lower total gas flow rates. Lowering the total gas rate from $900 \mathrm{SLH}\left(60 \% \mathrm{~N}_{2}\right)$ to $540 \mathrm{SLH}\left(20 \% \mathrm{~N}_{2}\right)$ did not affect the $\mathrm{CO}$ conversion, but increased the hydrocarbon production rate about $30 \%$. Lowering the $\mathrm{N}_{2}$ feed concentration to $12 \%$, and then to $6 \%$ decreased rather than increased the CO conversion. This run was terminated because the catalyst activity was too low. It was decided to install a smaller $\mathrm{N}_{2}$ mass flow meter to improve the accuracy at lower $\mathrm{N}_{2}$ feed rates. Since the $\mathrm{CO}$ and $\mathrm{H}_{2}$ conversions are calculated from both the mass flow meter rates and from G.C. analyses of the feed and product gases using $\mathrm{N}_{2}$ as an internal standard, it is necessary to get accurate low $\mathrm{N}_{2}$ flow rates.

Run No. 33 in the M3-SBCR was started on November 28th with a charge of $31.4 \mathrm{gm}$ of Catalyst No. Co.053-4. This was another attempt to obtain the highest $\mathrm{CO}$ conversion possible. A chilled water precooler coil was installed on the feed gas line to help heat removal during high conversion levels. The $\mathrm{CO}$ conversion at startup conditions was only $41.4 \%$, but was the same as Runs 29 and 30 in M3-SBCR. The M4-SBCR gave higher CO conversion, 50.4\%, for some unknown reason. In Period No. 2, the three gas feed rates were decreased proportionally to $60 \%$ of startup ( $540 \mathrm{SLH}$ ) and the reaction pressure was lowered to $300 \mathrm{psi}$. The CO conversion dropped to $35.2 \%$ and the $\mathrm{C}_{1}^{+}$production rate decreased $50 \%$. Lowering the total gas rate to $400 \mathrm{SLF}$ raised the $\mathrm{CO}$ conversion to $38.7 \%$. The $\mathrm{CO}$ conversion rose to $39.6 \%$ at $250^{\circ} \mathrm{C}$ reaction temperature, and dropped to $37.7 \%$ at $260^{\circ} \mathrm{C}$ temperature. Raising the pressure back to 450 psi only increased the $\mathrm{CO}$ conversion to $45.0 \%$. For Period 7, the $\mathrm{N}_{2}$ composition was lowered from 60 to $40 \%$. The CO conversion stayed the same, $45.7 \%$. Lowering the $\mathrm{N}_{2}$ composition to $25 \%$ increased the $\mathrm{CO}$ conversion to $49.2 \%$. The run conditions were changed to startup conditions. The $\mathrm{CO}$ conversion dropped to $16.1 \%$ vs. $41.4 \%$ in Period 1 . There was considerable loss of catalyst activity over the duration of this run, probably when the reaction temperature was raised to $260^{\circ} \mathrm{C}$.

Run No. 27 in the M4-SBCR was started on December 5th with a charge of $14.6 \mathrm{gm}$ of Catalyst No. Co.050. This catalyst contained $20 \%$ Co plus $0.5 \% \mathrm{Ru}, 8.5 \% \mathrm{Zr}$, and $0.1 \% \mathrm{~K}$ promoters on silica support. The $\mathrm{CO}$ conversion at startup conditions was $20.4 \%$, which is the same as obtained with Catalyst No. Co. 048 that contained $0.3 \% \mathrm{~K}$. The methane selectivities were also similar, approximately $10 \%$. The optimum amount of $\mathrm{K}$ to add for methane reduction without significantly lowering catalyst activity is somewhere between 0.1 and $0.3 \mathrm{wt} \%$.

Run No. 34 in the M3-SBCR was started on December 12th with a charge of $15.6 \mathrm{gm}$ of Catalyst No. Co. 060 . This catalyst contained $30 \mathrm{wt} \%$ Co on alumina support with no promoters. The CO conversion was $31.4 \%$, which is in the range that was expected without any metals or promoters added. The methane selectivity was approximately $14 \%$, which was also in the expected range.

During the shutdown period over the Christmas holiday, a number of routine maintenance checks were made. A smaller $\mathrm{N}_{2}$ mass flow meter was installed and calibrated on M4-SBCR. All other gas flow meters had calibration rechecks made and were found to be in good order. All necessary repairs were made on both SBCR reactor systems. 
APPENDIX D

Slurry Bubble Column Reactor Data 


\section{SUMMARY OF MB SBCR RUM RESULTS}

a) Unless otherwise noted, total flow is ca.15 L/min. STP, or $3 \mathrm{~cm} / \mathrm{sec}$ linear gas flow.

b) Conversion is cocal co conversion over the period ( $\pi$ ).

e) Conversion and selectivicies are calculated using 12 as an internal

standard in the GC analysis of the offgas.

d)Prod. race: Rate for producsion of total hydrocaricons, Cl+ $(\mathrm{kg} / \mathrm{kg} \mathrm{cat.,hr).}$

e)Alpha: Based on GC analysis of offgas and liquid prociuc:.

Run Cat. Cat. Per Time Time Temp Pres H2: Synil Conv.Prod. Selec:ivities Alpha Alpha Olefin/Paraf Olefins Coments

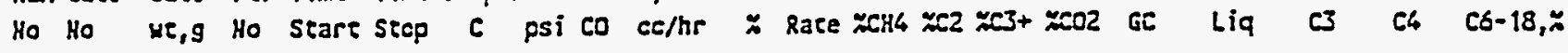

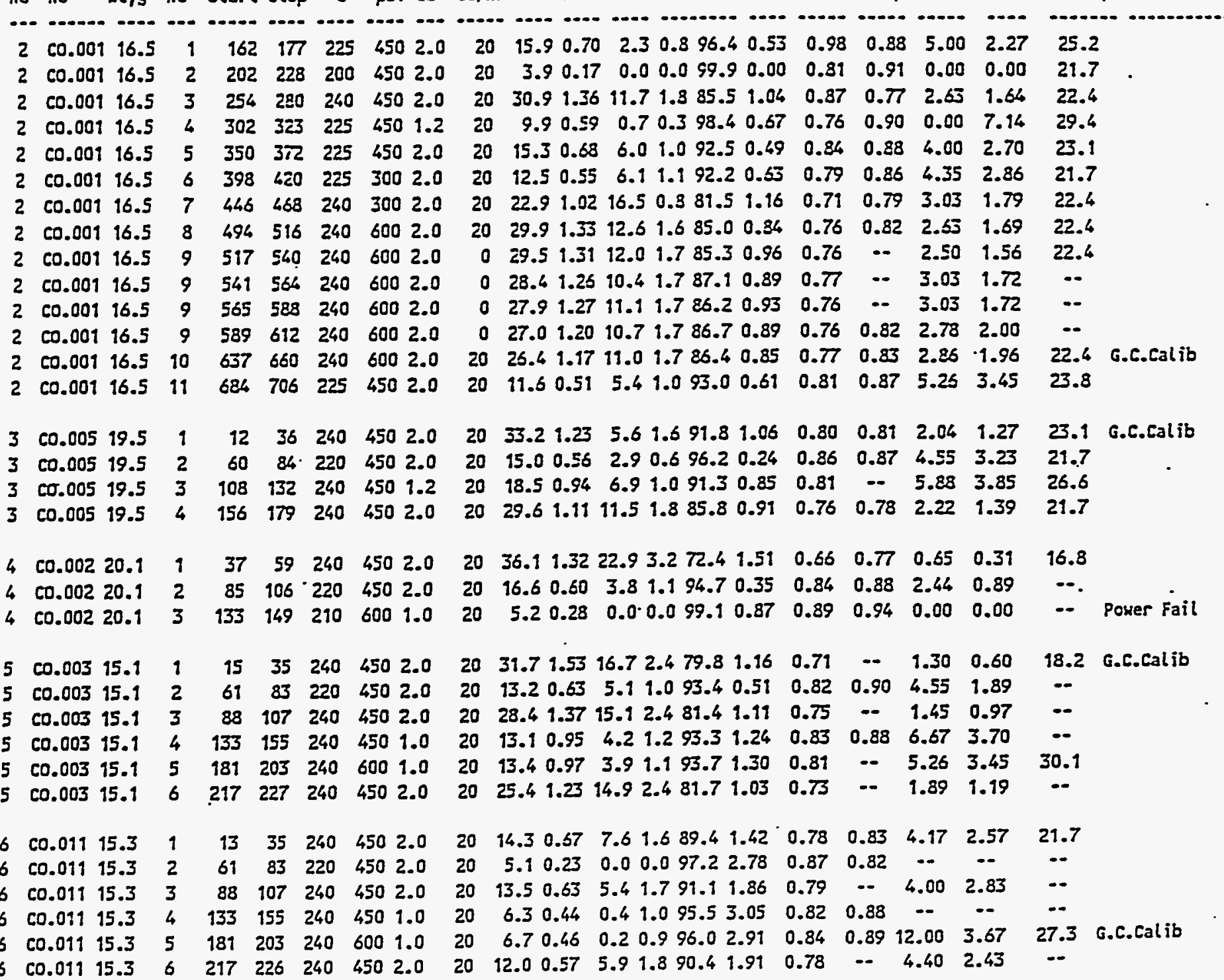


Table I

(Continued)

SUMMARY OF M3 SBCR RUN RESULTS

a) Unless otherwise noted, total flow is ca.15 L/min. STP, or $3 \mathrm{~cm} / \mathrm{sec}$ linear gas flow.

b) Conversion is total co conversion over the period (\%).

c) Conversion and selectivities are calculated using $N 2$ as an internal

standard in the GC analysis of the offgas.

d)Prod. race: Rate for production of total hydrocarbons, Cl+ (kg/kg cat., hr).

e)Alpha: Based on GC analysis of offgas and liquid product.

Run Cat. Cat. Per Time Time Temp Pres H2: Synfl Conv.Prod. Selectivities Alpha Alpha Olefin/Paraf Olefins Comments Ho Ho we,g Ho stare stop C psi CO cc/hr \% Rate zCH4 XC2 жc3+ zeO2 GC Liq C3 C4 C6-18,\%

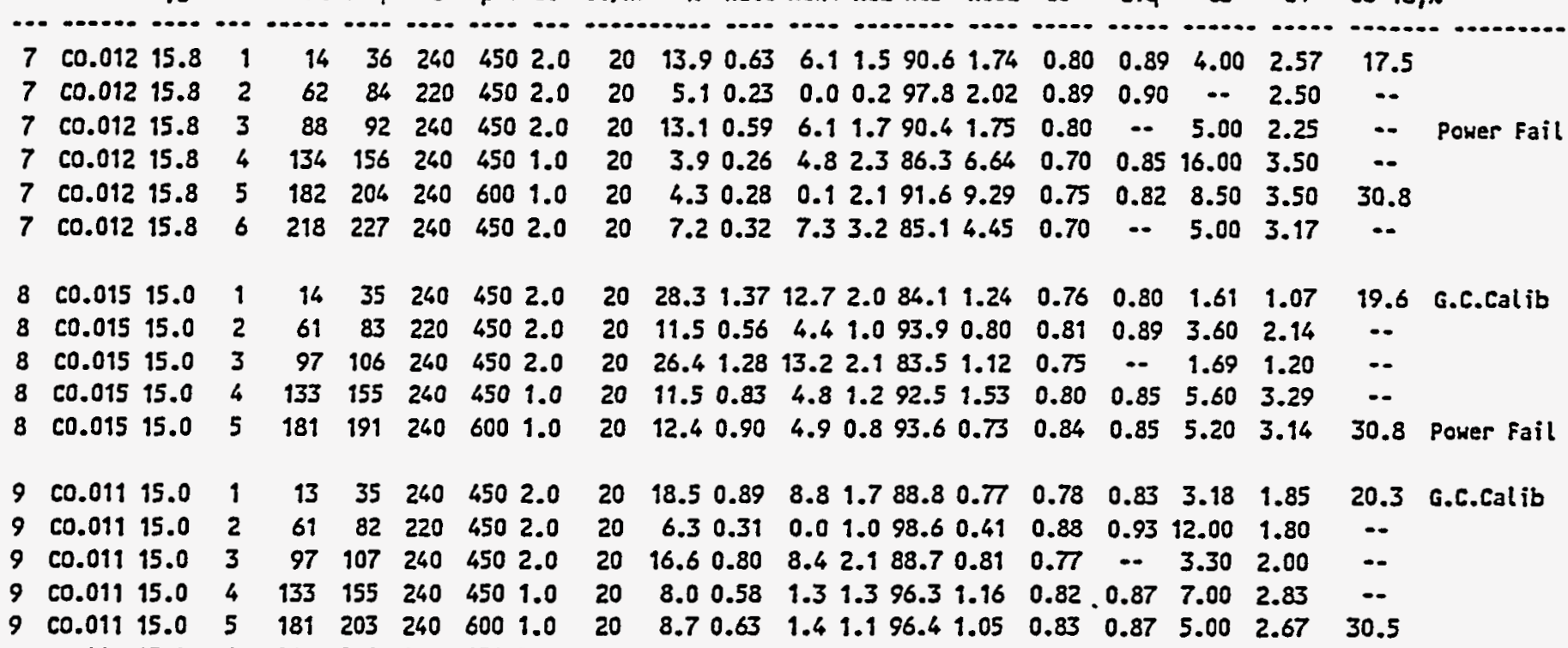

$9 \quad 0.01115 .0 \quad 6 \quad 228 \quad 229 \quad 240 \quad 4502.0$

$\begin{array}{lllllllllll}20 & 14.7 & 0.71 & 7.9 & 2.2 & 89.1 & 0.85 & 0.78 & \ldots & 3.78 & 1.67\end{array}$

-- Power Fail

$\begin{array}{rrrrrrrrr}11 & c 0.017 & 15.0 & 1 & 9 & 31 & 240 & 450 & 2.0 \\ 11 & c 0.017 & 15.0 & 2 & 55 & 79 & 220 & 450 & 2.0 \\ 11 & c 0.017 & 15.0 & 3 & 81 & 103 & 240 & 450 & 2.0 \\ 11 & c 0.017 & 15.0 & 4 & 129 & 151 & 240 & 450 & 1.0 \\ 11 & c 0.017 & 15.0 & 5 & 177 & 199 & 240 & 600 & 1.0 \\ 11 & c 0.017 & 15.0 & 6 & 213 & 221 & 240 & 450 & 2.0 \\ 12 & c 0.018 & 15.6 & 1 & 13 & 35 & 240 & 450 & 2.0 \\ 12 & c 0.018 & 15.6 & 2 & 61 & 80 & 220 & 450 & 2.0 \\ 12 & c 0.018 & 15.6 & 3 & 97 & 107 & 240 & 450 & 2.0 \\ 12 & c 0.018 & 15.6 & 4 & 133 & 155 & 240 & 450 & 1.0 \\ 2 & c 0.018 & 15.6 & 5 & 181 & 203 & 240 & 600 & 1.0 \\ 2 & 00.018 & 15.6 & 6 & 217 & 226 & 240 & 450 & 2.0\end{array}$

$\begin{array}{lllllllllllll}20 & 27.2 & 1.32 & 11.2 & 1.8 & 85.9 & 1.14 & 0.77 & 0.80 & 2.09 & 1.20 & 30.8\end{array}$

$\begin{array}{llllllllllll}20 & 11.4 & 0.55 & 5.0 & 0.0 & 94.8 & 0.22 & 0.88 & .0 .90 & 5.00 & 3.20\end{array}$

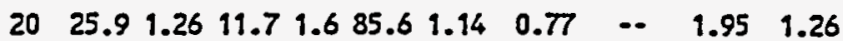

$\begin{array}{lllllllllll}20 & 11.6 & 0.84 & 5.0 & 0.0 & 93.8 & 1.19 & 0.82 & 0.86 & 25.0 & 2.88\end{array}$

$\begin{array}{lllllllllll}20 & 11.6 & 0.84 & 4.2 & 0.0 & 94.7 & 1.17 & 0.86 & 0.86 & 24.0 & 1.63\end{array}$

$\begin{array}{llllllllllll}20 & 24.3 & 1.20 & 12.1 & 1.4 & 86.4 & 0.08 & 0.78 & \ldots & 2.47 & 1.64\end{array}$

30.8

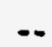

-.

21.0 G.C.Calib

$20 \quad 33.8 \quad 1.56 \quad 9.72 .0 \quad 86.6 \quad 1.68 \quad 0.75 \quad 0.85 \quad 1.81 \quad 1.13 \quad 21.0 \quad$ G.C.Calib

$\begin{array}{llllllllllllll}20 & 12.6 & 0.59 & 2.8 & 0.0 & 97.1 & 0.08 & 0.90 & 0.88 & \ldots & \ldots & \ldots\end{array}$

$\begin{array}{llllllllllll}20 & 27.9 & 1.30 & 9.4 & 1.9 & 87.7 & 1.03 & 0.75 & \ldots & 2.65 & 2.17 & \ldots\end{array}$

$\begin{array}{lllllllllllll}20 & 12.4 & 0.86 & 3.3 & 0.0 & 95.0 & 1.67 & 0.80 & 0.86 & \ldots & \ldots & \ldots\end{array}$

$\begin{array}{llllllllllll}20 & 13.0 & 0.90 & 3.2 & 0.0 & 94.9 & 1.86 & 0.80 & 0.85 & \ldots & \ldots & 38.5\end{array}$

$2 \quad 0.01815 .6$

$\begin{array}{llllllllllll}20 & 26.4 & 1.22 & 10.1 & 1.4 & 86.8 & 1.74 & 0.73 & \ldots & 2.89 & 2.05 & \ldots\end{array}$ 
Table I

(Continued)

\section{SUMMARY OF HZ SBCR RUM RESULTS}

a) Unless otherwise noted, total $\mathrm{flow}$ is $\mathrm{ca} .15 \mathrm{~L} / \mathrm{min}$. $5 \mathrm{TP}$, or $3 \mathrm{~cm} / \mathrm{sec}$ linear gas floh.

b) Conversion is total co conversion over the period $(\%)$.

c) Conversion and selectivities are calculated using $H 2$ as an internal

standard in the GC analysis of the offgas.

d)Prod. rate: Rate for production of total hydrocarbons, Cl+ $(\mathrm{kg} / \mathrm{kg} \mathrm{cat.,} \mathrm{hr).}$

e)Alpha: Based on GC analysis of offgas and liquid product.

Run Cat. Cat. Per Time Time Temp Pres $42:$ Synfl Conv.Prod. Selectivities Alpha Alpha Olefin/Paraf olefins Comments

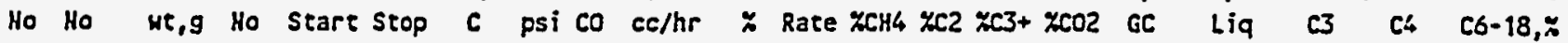

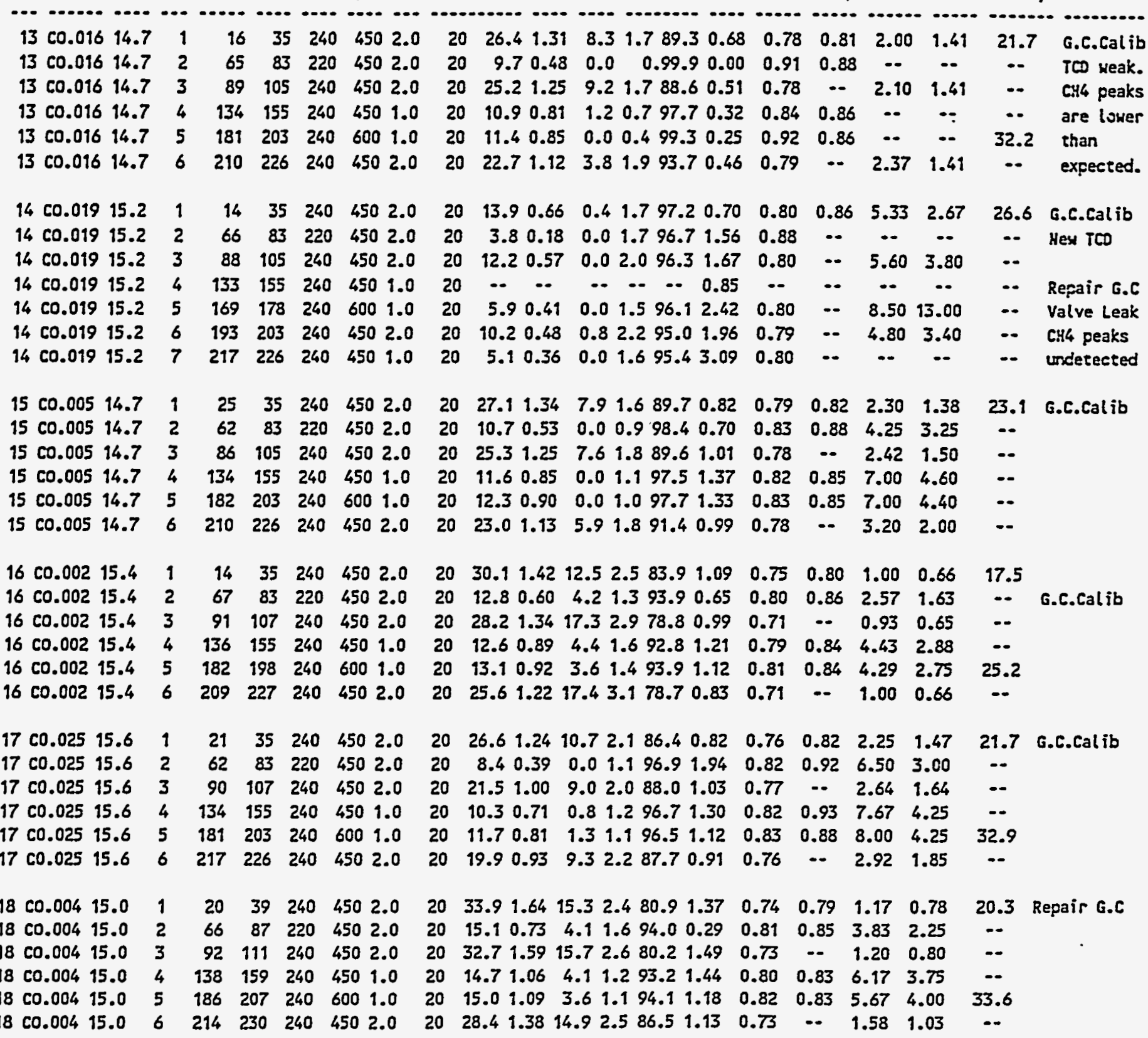




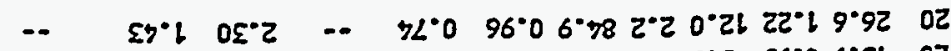

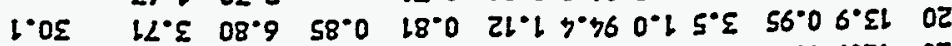

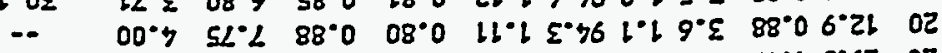

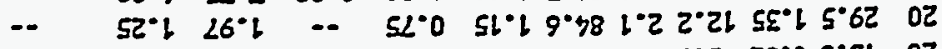

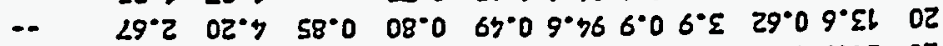

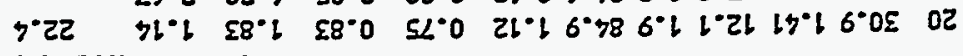

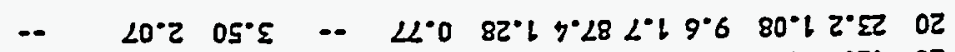

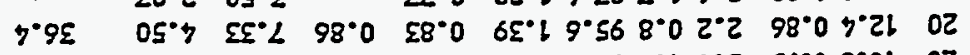

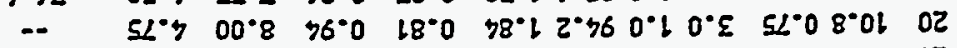

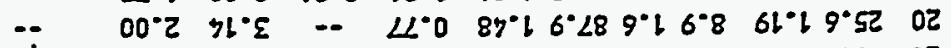

$\therefore \quad 29^{\circ} 700^{\circ} \Sigma \quad 68^{\circ} 0 \quad 92^{\circ} 0 \quad 20^{\circ} 15^{\circ} 962^{\circ} \mathrm{L} 5^{\circ} \mathrm{L} \quad 75^{\circ} 07^{\circ} \mathrm{L} \quad 02$

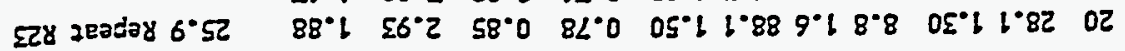

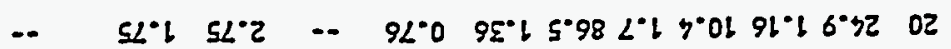

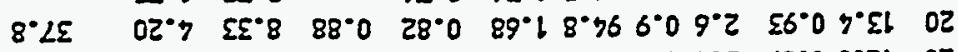

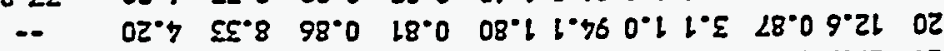

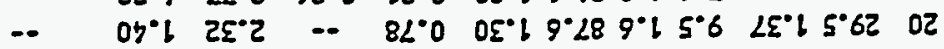

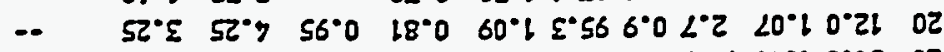

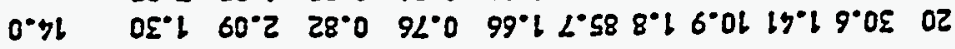

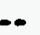

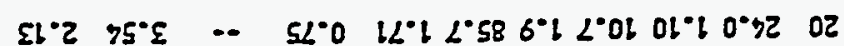
$09^{\circ} \varepsilon \quad 00^{\circ} \mathrm{L} \quad 28^{\circ} 0 \quad 28^{\circ} 0 \quad 10^{\circ} 29^{\circ} \geqslant 68^{\circ} 09^{\circ} \mathrm{L} \quad 18^{\circ} 08^{\circ} \mathrm{Lb} \quad 02$

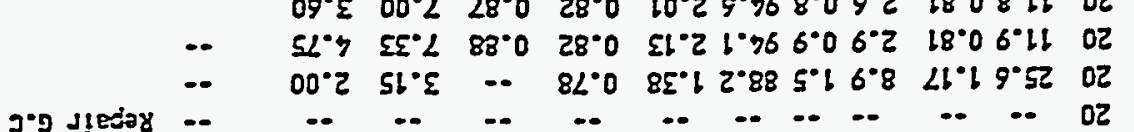

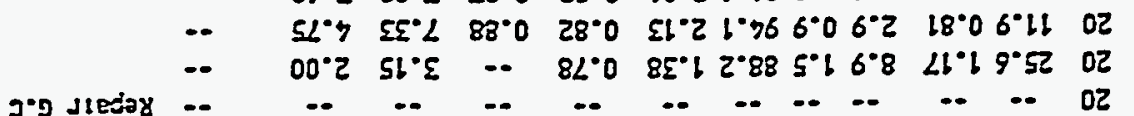

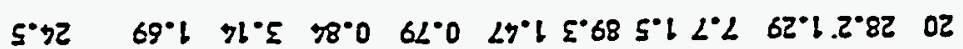

unop anes d!

$\cdots$

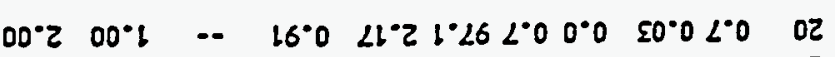

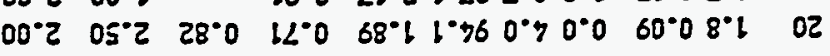

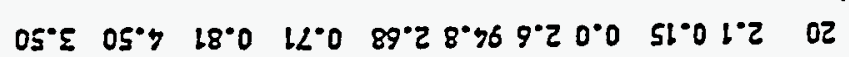

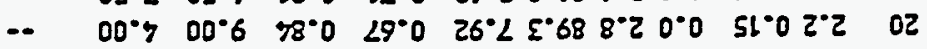

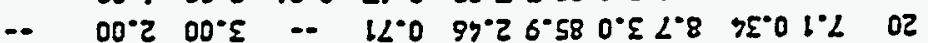

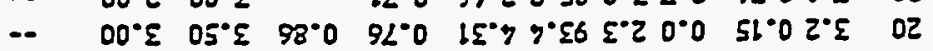

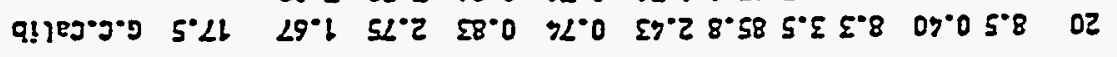

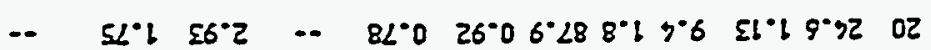

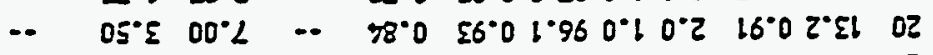

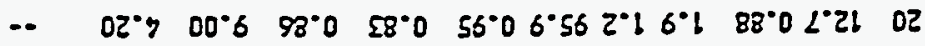

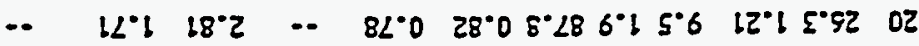

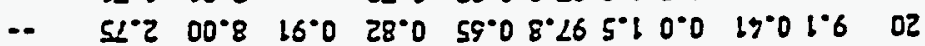

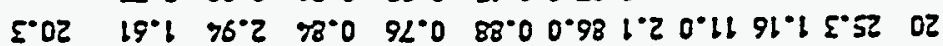

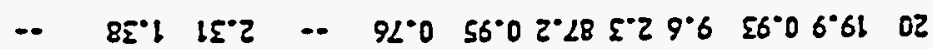

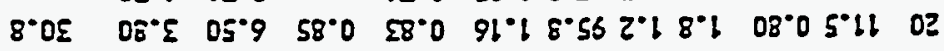

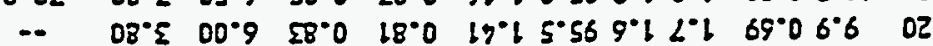

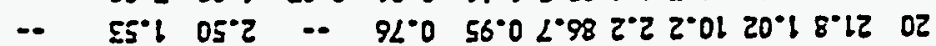

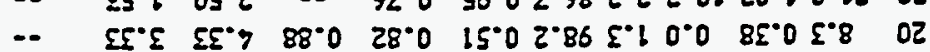

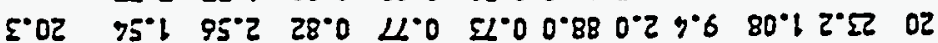

0.2 057 052 052 122 9 0.1009 032 $202 \quad 681.5$ $0 \div 1$ O5Y 052 65l Lbl 0.2057 072 $46 \mathrm{E} \quad \varepsilon$ 0.2 057 $022 \quad 28 \quad 29$ 0.2 057 052 6E 61

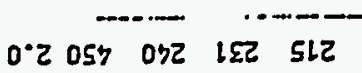
0.1009 002 202 68L $0 \% 1$ OSH OSर 651 ह\$ 0.2 05y 042 65L 26 0.2 05y 022 69 55 0.2 057 0म2 हE 6 b

0.2 057 0\%ट IEZ HL $0 \% 1009$ O\$2 LOZ L8L 0.1 057 0D2 65L 6EL 0.2 057 072 16456 0.2 05y 0Z2 L8 LL 0.2 O5马 OSZ GE 61

0.2 05t 052 922 st2 0.1009 0\$2 $202 \quad \angle 81$ 0.1 05\% 052 65t l9t 0.2 054 032 $346 \quad 76$ 0.2 OSY 0Z2 0.2 057 052 $65 \quad 12$

0.2 05y 0टz ह9 हम 0.2054 032 2566 $0 \div 1009072902986$ 0.1 057 0\$2 LSL 8EL 0.2 057 052 016 56 0.2 057 $022 \quad 98 \quad 89$ 0.2 057 032 $85 \quad 72$

0.2 057 072 502 561 $0 \% 1009$ 072 8Lb 696 $0 \% 1$ 057 052 871 951 0.2 057 052 60606 0.2 057 032 51 99 0.2 05y OHZ LE Ll $6^{\circ} 51 \quad 150^{\circ} 03$ $6^{\circ} \mathrm{St} 150^{\circ} 03$ $6^{\circ}$.5t $150^{\circ} 05$ $6^{\circ} 5 ! \quad 150.05$ $6^{\circ} 5 t \quad\left\lfloor 50^{\circ} 0 J\right.$ $6^{\circ} 5 ! \quad 150^{\circ} 055$

$9^{\circ} 51<70^{\circ} 035$ $g^{\circ} \mathrm{sl} \angle 40^{\circ} 00 \mathrm{Si}$ $9^{\circ} 51 \angle 70^{\circ} 035 i$ $\varepsilon 9^{\circ} 51 \angle 30^{\circ} 0 J 5 i$ 2 9.5l $\angle 70^{\circ} 005 i$ 1 9.51 $290^{\circ} 005$

$9.516 \% 0^{\circ} 05$ \$2 $9^{\circ}$ St $670^{\circ} 03$ कर . $9.56670^{\circ} 03$ \%2 9.56 $670^{\circ} 05$ \$2 $29.51670^{\circ} 0532$ $199^{\circ} 51670^{\circ} 052$

$8^{\circ} 51 \angle 70^{\circ} 00 \Omega$ $58.51 \angle 90^{\circ} 00 \Omega$ $78^{\circ} 56 \angle 70^{\circ} 0 J \varepsilon 2$ \& $8^{\circ} 56 \quad 230^{\circ} 0 J$ EL $28^{\circ} \mathrm{SL} \angle 90^{\circ} 03 \Omega$ $1 \quad 8^{\circ}$. $\angle 70^{\circ} 0 J \Sigma$

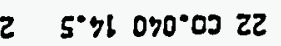
$15.91070^{\circ} 0022$

$5 \quad b^{\circ} 5 t$ \$i००0J 12 $\rightarrow 1.51 \% 10.0512$ E $1.51760^{\circ} 0012$ $2 \quad 1.51310^{\circ} 0012$ 1 1.5t $\$ 60^{\circ} 0 J$ LC

. $8.51 \quad 170^{\circ} 0302$ $8.51 \quad 170^{\circ} 0002$ $8^{\circ} \mathrm{SL} 170^{\circ} 00 \mathrm{OZ}$ $8^{\circ} \mathrm{sl} 170^{\circ} 0002$ $8.51170^{\circ} 0002$ $8^{\circ} 51 \quad 170^{\circ} \mathrm{CO} 02$

0.2057 072 $522 \quad 012 \quad 9 \quad 9.56 \quad 120^{\circ} 0361$ $0 \% 1009$ 072 $502 \quad 286 \quad 5 \quad 9^{\circ} 51 \quad 120^{\circ} 0361$ 0.1 057 0D2 5SL गEL Y $9^{\circ} 51 \quad 120^{\circ} 0061$ 0.2 057 032 LOt 06 E $9^{\circ} 51 \quad 120^{\circ} 0566$ 0.2 057 $022 \quad 58 \quad 29 \quad 2 \quad 9^{\circ} 51 \quad 120^{\circ} 0261$ 0.2 057 032 SE ol $1 \quad 9^{\circ} 51 \quad 120^{\circ} 0361$

$z^{2} 81-95$ כ כ כ 5 b

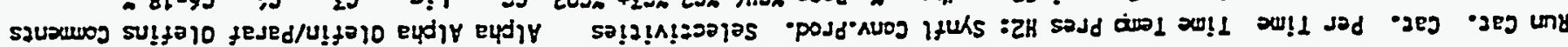

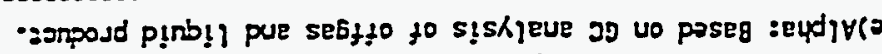

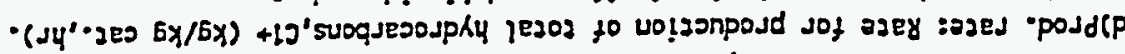

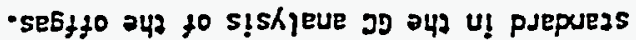

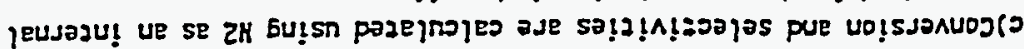

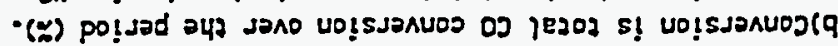

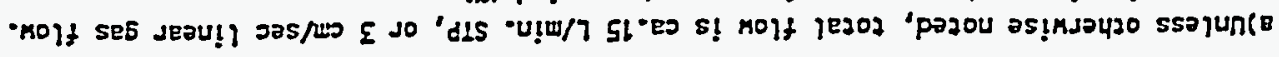

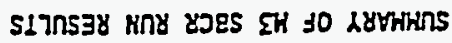


Table I

(Continued)

SUMARY OF MB SBCR RUK RESULTS

a) Unless otherwise noted, total flow is ca. $15 \mathrm{~L} / \mathrm{min}$. STP, or $3 \mathrm{~cm} / \mathrm{sec}$ linear gas flow.

b)Conversion is total co conversion over the period (ü).

c)Conversion and selectivities are calculated using $\mathrm{HZ}$ as an internat

standard in the GC analysis of the offgas.

d)Prod. rate: Rate for produc:ion of total hydrocarbons, Cl+ $(\mathrm{kg} / \mathrm{kg}$ cat., hr).

e)Alpha: Based on GC analysis of offgas and liquid product.

RLn Cat. Cat. Per Time Time Temp Pres H2: Synfl Conv.Prod. Selectivities Alpha Alpha Olefin/Paraf Olefins Comments

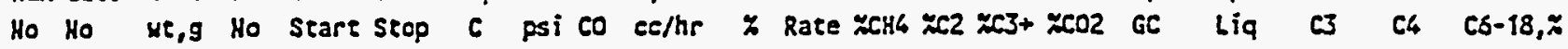

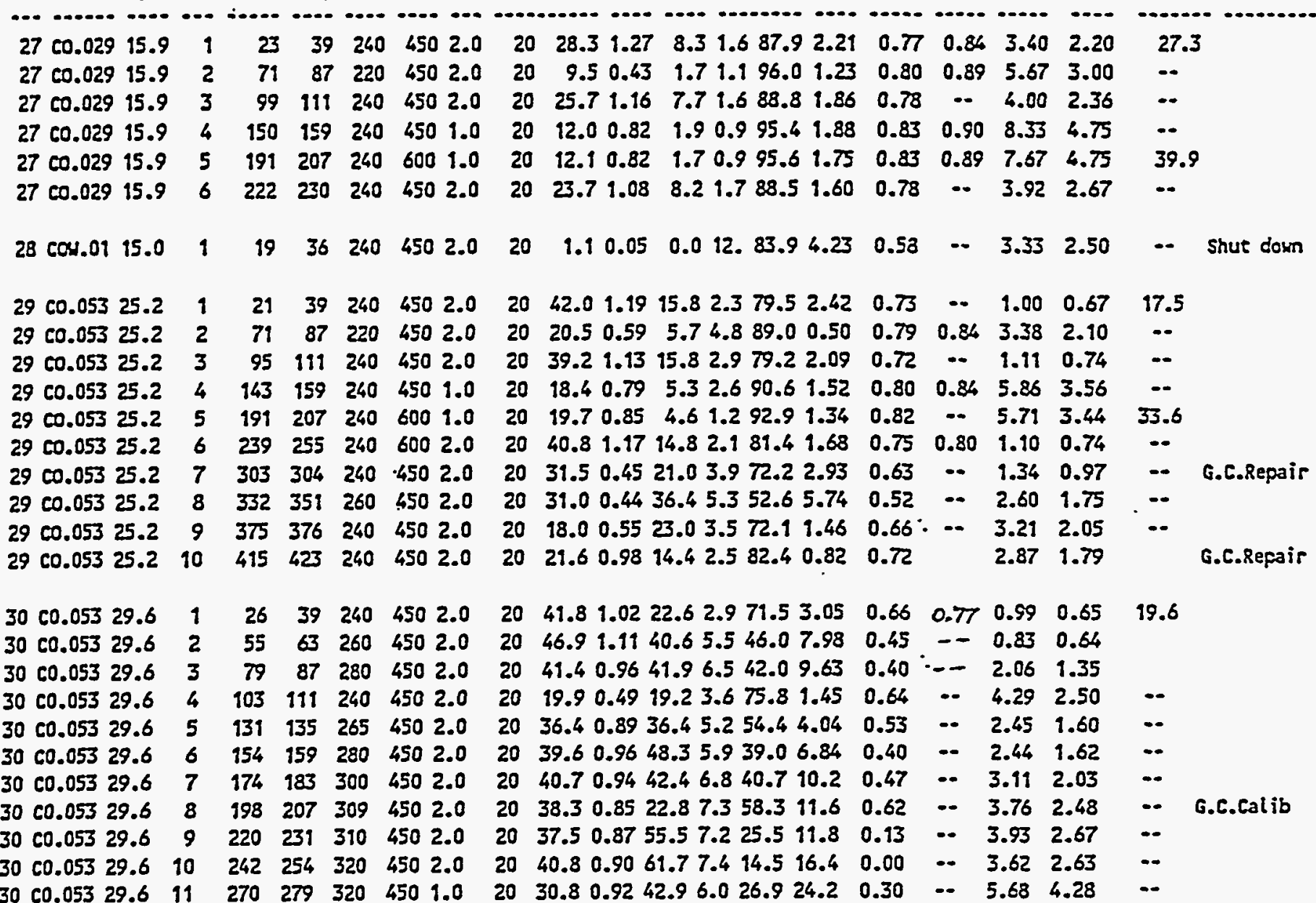

$30 \mathbf{c 0 . 0 5 3} 29.6 \quad 11$ 
Table I

(Continued)

\section{SUMMARY OF M3 SBCR RUN RESULTS}

a) Unless otherwise noted, total flow is ca. $15 \mathrm{~L} / \mathrm{min}$. STP, or $3 \mathrm{~cm} / \mathrm{sec}$ linear gas flow.

b)Conversion is total co conversion over the period ( $\%$ ).

c) Conversion and selectivities are calculated using $\mathrm{H} 2$ as an internal

standard in the GC analysis of the offgas.

d)Prod. rate: Rate for production of total hydrocarbons, Cl+ $(\mathrm{kg} / \mathrm{kg}$ cat., hr).

e)Alpha: Based on GC analysis of offgas and liquid product.

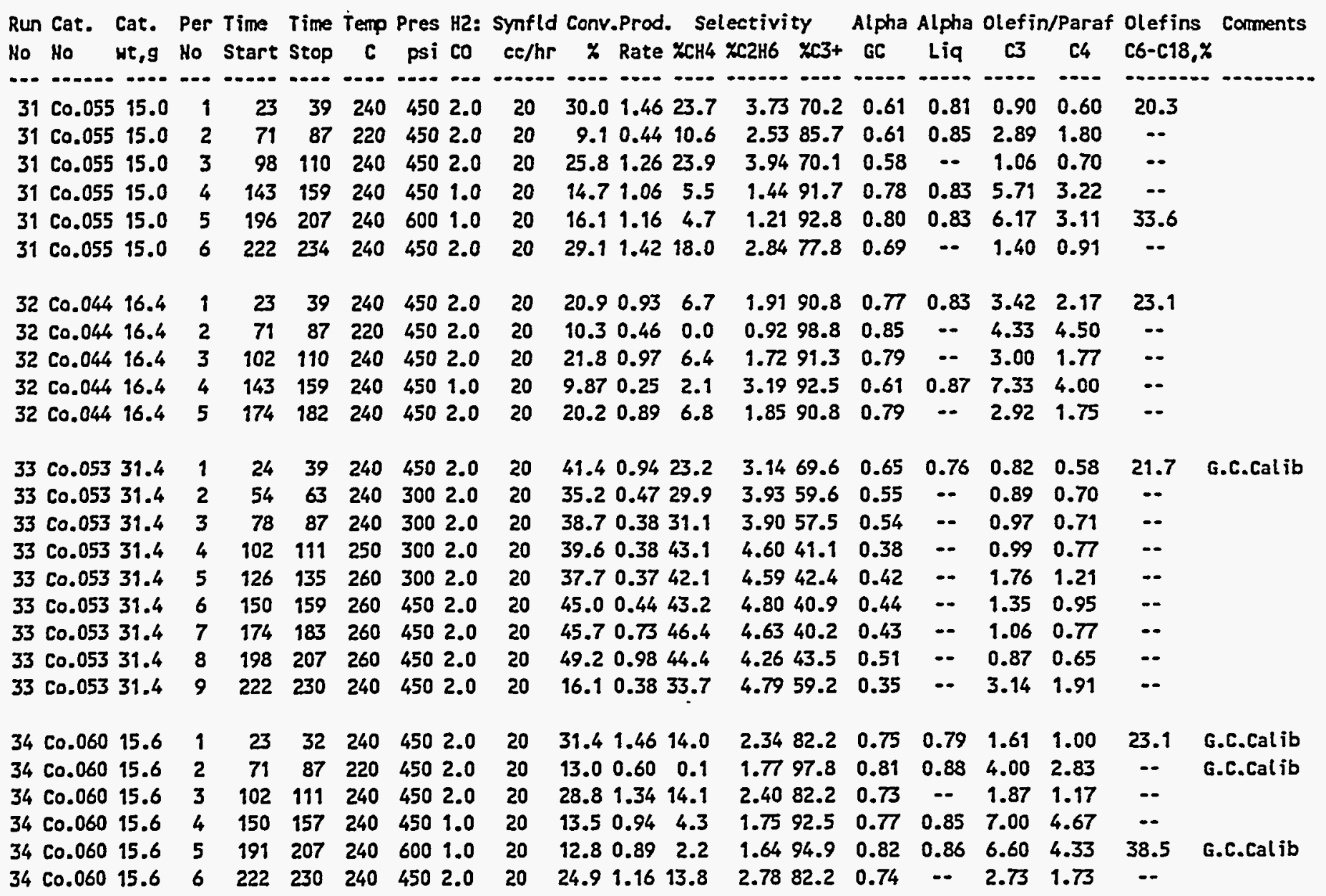




\begin{tabular}{cccc} 
Period No. & Temp. & Pres. & H2/CO Ratio \\
\hline 1 & $240 \mathrm{C}$ & $450 \mathrm{psi}$ & 2.0
\end{tabular}

a) Total flow is ca. $15 \mathrm{~L} / \mathrm{min}$. STP, or $3 \mathrm{~cm} / \mathrm{sec}$ linear gas flow. Flows: N2-563 SLH, H2-225 SLH, CO-112.5 SLH.

b) Conversion is total $c 0$ conversion over the period ( $(z)$.

c) Conversion and selectivities are calculated using $\mathrm{N} 2$ as an internal standard in the GC analysis of the offgas.

d) Prod. rate: Rate for production of total hydrocarbons $\mathrm{C}+\mathrm{I}(\mathrm{kg} / \mathrm{kg}$ cat., hr). e) Alpha: Based on GC analysis of offgas and liquid product.

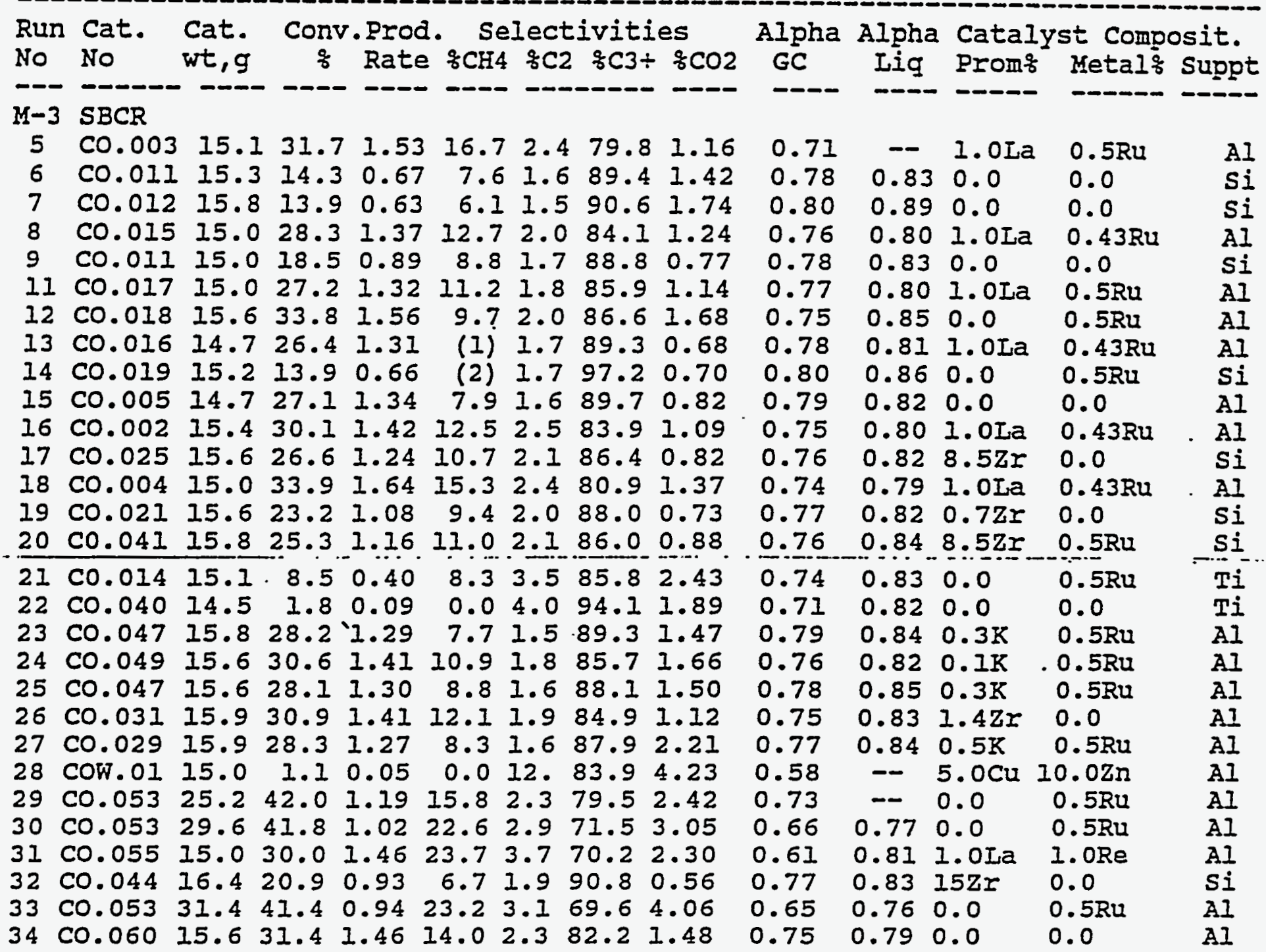

Note: Catalyst Run 9 screened thru 170 x 400 mesh. Catalysts For Runs 11 through 28 (except Runs 21 and 22) screened thru 150x 400 mesh. Catalysts for Runs 21 and 22 screened thru $100 \times 400$ mesh. Catalysts Nos. Co.029 and 0.060 contained 30 wt\% cobalt.

(I) Weak TCD filaments in G.C. CH4 peaks smaller than expected.

(2) Internal valve in G.C. leaked. $\mathrm{CH} 4$ peak undetected. 
Table III

M3 SBCR RUN RESUITS

COMPARISON OF CATALYST ACTIVITY AT SIMILAR RUN CONDITIONS

\begin{tabular}{cccc} 
Period No. & Temp. & Pres. & H2/CO Ratio \\
\hline 2 & $220 \mathrm{C}$ & $450 \mathrm{psi}$ & $\mathbf{2 . 0}$
\end{tabular}

a) Total flow is ca. $15 \mathrm{I} / \mathrm{min}$. STP, or $3 \mathrm{~cm} / \mathrm{sec}$ linear gas flow.

Flows: $\mathrm{N2}-563 \mathrm{SLH}, \mathrm{H} 2-225 \mathrm{SLH}, \mathrm{CO}-112.5 \mathrm{SLH}$

b) Conversion is total co conversion over the period ( $\%$ ).

c) Conversion and selectivities are calculated using N2 as an internal standard in the GC analysis of the offgas.

d) Prod. rate: Rate for production of total hydrocarbons, Cl+ (kg/kg cat.,hr) e) Alpha: Based on GC analysis of offgas and liquid product.

\begin{tabular}{|c|c|c|c|c|c|c|c|c|c|c|c|c|c|}
\hline $\begin{array}{l}\text { Run } \\
\text { No }\end{array}$ & $\begin{array}{l}\text { Cat. } \\
\text { No }\end{array}$ & $\begin{array}{l}\text { Cat. } \\
\text { wt, g }\end{array}$ & $\begin{array}{c}\text { Conv } \\
\frac{\circ}{6}\end{array}$ & $\begin{array}{l}\text { Prod. } \\
\text { Rate }\end{array}$ & $\begin{array}{r}\mathrm{Se} \\
\mathrm{r} \mathrm{CH} 4\end{array}$ & $\begin{array}{l}\text { ect: } \\
\text { sc2 }\end{array}$ & $\begin{array}{l}\text { viti } \\
\text { \&C3t }\end{array}$ & $\begin{array}{l}\text { s } \\
\& \mathrm{CO} 2\end{array}$ & $\begin{array}{l}\text { Alpha } \\
\text { GC }\end{array}$ & $\begin{array}{l}\text { Alpha } \\
\text { Iiq }\end{array}$ & $\begin{array}{c}\text { Olefir } \\
\text { C3 }\end{array}$ & $\begin{array}{l}\text { Paraf } \\
\text { C4 }\end{array}$ & $\begin{array}{l}\text { olefin } \\
\text { c6-18, }\end{array}$ \\
\hline- & $-\infty-\infty$ & & - & - & $-\infty$ & & -- & --- & . & & $-\infty-$ & 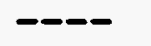 & \\
\hline 5 & $\mathrm{CO} .003$ & 15.1 & 13.2 & 0.63 & 5.1 & 1.0 & 93.4 & 0.5 & 0.82 & 0.90 & 4.55 & 1.89 & \\
\hline 6 & $\mathrm{Co} .011$ & 15.3 & 5.1 & 0.23 & 0.0 & 0.0 & 97.2 & 2.8 & 0.87 & .82 & - & - & \\
\hline 7 & $\mathrm{CO} .012$ & 15.8 & 5.1 & 0.23 & 0.0 & .2 & 97.8 & 2.0 & 0.89 & 0.90 & -- & 2.50 & \\
\hline 8 & $\mathrm{Co} .015$ & 5.0 & 11.5 & 0.56 & 4.4 & .0 & 93.9 & 0.8 & 0.81 & 0.89 & 3.60 & 2.14 & \\
\hline 9 & $\mathrm{co} 0.011$ & 5.0 & 6.3 & 0.31 & 0.0 & .0 & 98.6 & 0.4 & 0.88 & 0.93 & 12.00 & 1.80 & \\
\hline 11 & $\mathrm{Co} .017$ & 5.0 & 11.4 & 0.55 & 5.0 & .0 & 94.8 & 0.2 & 0.88 & 0.90 & 5.00 & 3.20 & \\
\hline 12 & $\mathrm{Co} .018$ & 15.6 & 12.6 & 0.59 & 2.8 & .0 & 97.1 & 0.1 & 0.90 & 0.88 & -- & -- & \\
\hline 13 & $\mathrm{Co.016}$ & ; 14.7 & 9.7 & 0.48 & (I) & 0.1 & 99.9 & 0.0 & 0.91 & 0.88 & -- & -- & \\
\hline 14 & $\mathrm{Co} .019$ & 15.2 & 3.8 & 0.18 & (2) & 1.7 & 96.7 & 1.6 & 0.88 & - & - & $-\infty$ & \\
\hline 15 & $\mathrm{CO} .005$ & $; 14.7$ & 10.7 & 0.53 & 0.0 & 0.9 & 98.4 & 0.7 & 0.83 & 0.88 & 4.25 & 3.25 & \\
\hline 16 & $\mathrm{CO} .002$ & 15.4 & 12.8 & 0.60 & 4.2 & 1.3 & 93.9 & 0.7 & 0.80 & 0.86 & 2.57 & 1.63 & \\
\hline 17 & $\mathrm{CO} .025$ & ; 15.6 & 8.4 & 0.39 & 0.0 & & 96.9 & 1.9 & 0.82 & 92 & 6.50 & 3.00 & \\
\hline 18 & $\mathrm{CO} .004$ & & 15.1 & 0.73 & & & .0 & 0.3 & 0.81 & 85 & 3.83 & 2.25 & \\
\hline 19 & $\mathrm{CO} .021$ & 15.6 & 8.3 & 0.38 & 0.0 & 1.3 & 98.2 & 0.5 & 0.82 & 0.88 & 4.33 & 3.33 & \\
\hline 20 & $\mathrm{Co} .041$ & 15.8 & 9.1 & 0.41 & 0.0 & 1.5 & 97.8 & 0.7 & 0.82 & 91 & 8.00 & 2.75 & \\
\hline 21 & Co. 014 & 15.1 & 3.2 & 0.15 & 0.0 & 2.3 & 85.8 & 4.3 & 0.76 & 0.86 & 3.50 & 3.00 & \\
\hline 22 & $\mathrm{Co} .040$ & 14.5 & 0.7 & 0.35 & 0.0 & 0.7 & 97.1 & 2.2 & 0.91 & - & 1.00 & 2.00 & \\
\hline 23 & $\mathrm{CO} .047$ & 15.8 & -- & --- & (3) & - & -ー- & $-\infty$ & --- & -- & $---\infty$ & $-\cdots$ & \\
\hline 24 & Co. 049 & 15.6 & 12.0 & 1.07 & 2.7 & & 95.3 & 1.1 & 0.81 & 0.95 & 4.25 & 3.25 & \\
\hline - & Co.047 & 15.6 & 7.4 & 0.34 & 1.5 & & 96.3 & 1.0 & 0.76 & 0.89 & 3.00 & 4.67 & - \\
\hline 26 & $c 0.031$ & 15.9 & 13.6 & 0.62 & 3.9 & & 94.6 & 0.5 & 0.80 & 0.85 & 4.20 & 2.67 & 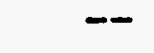 \\
\hline 27 & $\mathrm{CO} .029$ & 15.9 & 9.5 & 0.43 & 1.7 & $I . I$ & 96.0 & 1.2 & 0.80 & 0.89 & 5.67 & 3.00 & \\
\hline 29 & $\mathrm{CO} .053$ & 25.2 & 20.5 & 0.59 & 3.1 & 4.8 & 89.0 & 0.5 & 0.79 & 0.84 & 3.38 & 2.10 & \\
\hline 31 & $\mathrm{CO} .055$ & 15.0 & 9.1 & 0.44 & 10.6 & 2.5 & 85.7 & 1.1 & $0.6 I$ & 0.85 & 2.89 & 1.80 & 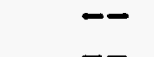 \\
\hline 3 & $\begin{array}{l}C 0.044 \\
C 0.060\end{array}$ & 16.4 & 10.3 & $\begin{array}{l}0.46 \\
0.60\end{array}$ & $\begin{array}{l}0.0 \\
0.1\end{array}$ & & $\begin{array}{l}98.8 \\
97.8\end{array}$ & 0.3 & $\begin{array}{l}0.85 \\
0.87\end{array}$ & $0 .-\overline{88}$ & 4.33 & $\begin{array}{r}4.50 \\
2.83\end{array}$ & - \\
\hline & $\mathrm{CO} .0$ & & & & 0.1 & & & 0.4 & 81 & 0.88 & 4.00 & 2.83 & \\
\hline
\end{tabular}

Note: Catalyst Run 9 screened thru $170 \times 400$ mesh.

Catalysts for Runs 11 thru 27 (except Runs 21 and 22) screened through $150 \times 400$ mesh.

Catalysts for Runs $2 I$ and 22 screened thru $100 \times 400$.

(1) Weak TCD filaments in G.C. CH4 peaks smaller than expected.

(2) Internal valve in G.C. leaked. $\mathrm{CH} 4$ peak undetected.

(3) Replaced dual column restrictor valve. 
Table IV

DATE: $12 / 30 / 94$

M3 SBCR RUN RESUITS

COMPARISON OF CATALYST ACTIVITY AT SIMILAR RUN CONDITIONS

\begin{tabular}{cccc} 
Period No. & Temp. & Pres. & H2/CO Ratio \\
\hline 4 & $240 \mathrm{C}$ & $450 \mathrm{psi}$ & 1.0
\end{tabular}

a) Total flow is ca. $15 \mathrm{~L} / \mathrm{min}$. STP, or $3 \mathrm{~cm} / \mathrm{sec}$ linear gas flow. Flows: N2-562 SIH, H2-119 SIH, CO-II9 SIH

b) Conversion is total co conversion over the period ( $\%$ ).

c) Conversion and selectivities are calculated using $\mathrm{N} 2$ as an internal standard in the GC analysis of the offgas.

d) Prod. rate: Rate for production of total hydrocarbons, clt (kg/kg cat.,hr) e) Alpha: Based on GC analysis of offgas and liquid product.

\begin{tabular}{|c|c|c|c|c|c|c|c|c|c|c|c|c|c|}
\hline $\begin{array}{l}\text { Run } \\
\text { No }\end{array}$ & $\begin{array}{l}\text { Cat. } \\
\text { No }\end{array}$ & $\begin{array}{l}\text { Cat. } \\
\text { wt, g }\end{array}$ & $\begin{array}{c}\text { Conv } \\
q\end{array}$ & $\begin{array}{l}\text { Prod. } \\
\text { Rate }\end{array}$ & $\begin{array}{c}\text { Sel } \\
\% \mathrm{CH} 4\end{array}$ & \% $\mathrm{C} 2$ & $8 \mathrm{c} 3+$ & $\begin{array}{l}\text { es } \\
\text { \&cO2 }\end{array}$ & $\begin{array}{l}\text { Alpha } \\
\text { GC }\end{array}$ & $\begin{array}{l}\text { Alpha } \\
\text { Iiq }\end{array}$ & $\begin{array}{c}\text { Olefi } \\
\text { C3 }\end{array}$ & $\begin{array}{l}\text { Paraf } \\
\text { C4 }\end{array}$ & c6-18, \\
\hline 3 & $c 0.003$ & 15.1 & 13.1 & 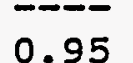 & & & & 1.2 & 10 & & 6.67 & & \\
\hline 6 & Co.0II & 15.3 & $\begin{array}{r}6.1 \\
6.3\end{array}$ & 44 & $\begin{array}{l}4.2 \\
0.4\end{array}$ & & 95.5 & 3.1 & 0.82 & $\begin{array}{l}0.88 \\
0.88\end{array}$ & $\begin{array}{c}6.67 \\
--\end{array}$ & $\begin{array}{c}3.70 \\
---\end{array}$ & - \\
\hline 7 & $c 0.012$ & 15.8 & 3.9 & 0.26 & 4.8 & & 86.3 & 6.6 & 0.70 & 0.85 & 16.00 & 3.50 & \\
\hline 8 & $C 0.015$ & 15.0 & 11.5 & 0.83 & 4.8 & 1. & 92.5 & 1.5 & 0.80 & 0.85 & 5.60 & 3.29 & \\
\hline 9 & 00.011 & 15.0 & 8.0 & 0.58 & 1.3 & 10 & 96.3 & 1.2 & 0.82 & .87 & 7.00 & 2.83 & \\
\hline 11 & $\mathrm{Co.017}$ & 15.0 & 11.6 & 0.84 & 5.0 & 0 & 93.9 & 1.2 & 0.82 & 86 & 25.00 & 2.88 & \\
\hline 12 & $C 0.018$ & 15.6 & 12.4 & 0.86 & 3.3 & 0 & 95.0 & 1.7 & 0.80 & 0.86 & - & -- & \\
\hline 13 & $c 0.016$ & 14.7 & 10.9 & 0.81 & (I) & & 97.7 & 0.3 & 0.84 & 0.86 & - & - & \\
\hline 14 & Co.019 & 15.2 & -- & - & (2) & & -- & - & 0.80 & -- & - & - & \\
\hline 15 & $\mathrm{CO} .005$ & 14.7 & 11.6 & 0.85 & 0.0 & 1.1 & 97.5 & 1.4 & 0.82 & 0.85 & 7.00 & 4.60 & \\
\hline 16 & $\mathrm{CO} .002$ & 15.4 & 12.6 & 0.89 & 4.4 & 1.6 & 92 & 1.2 & .79 & 3 & 4.43 & 2.88 & \\
\hline 17 & $\mathrm{CO} .025$ & ; 15.6 & 10.3 & 0.71 & 0.8 & 1.2 & 96.7 & 1.3 & 82 & 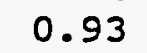 & 7.67 & 4.25 & \\
\hline 18 & $\mathrm{CO} .004$ & 15.0 & 14.7 & 1.06 & & 1.2 & 93.2 & 1.4 & 0.80 & 53 & 6.17 & 3.75 & - \\
\hline 19 & $\mathrm{Co.021}$ & 15.6 & 9.9 & 0.69 & 1.7 & 1.4 & 95.5 & 1.4 & 0.81 & 0.83 & 6.00 & 3.80 & . \\
\hline 20 & Co.04I & 15.8 & 12.7 & 0.88 & 1.9 & 1.2 & 95.9 & 1.0 & 0.83 & 0.86 & 9.00 & 4.20 & - \\
\hline 21 & $\mathrm{CO} .014$ & 15.1 & 2.2 & 0.15 & 0.0 & 2.8 & 89. & 7.9 & 0.67 & 0.84 & 9.00 & 4.00 & --7 \\
\hline 23 & $C 0.047$ & & 11.9 & 0.81 & 2.9 & 0.9 & 94.1 & 2.1 & 0.82 & 0. & 7.33 & 4.75 & -- \\
\hline 24 & $C 0.049$ & 6 & 12.6 & 0.87 & 3.1 & 1.0 & 94.1 & 1.8 & 0.81 & 0 . & 8.33 & 4.20 & 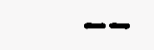 \\
\hline 25 & $\mathrm{CO} .047$ & & 10.8 & 0.75 & 2.0 & 1.0 & 94 & 1.8 & 0.81 & & 8.00 & 4.75 & -- \\
\hline 26 & Co.03I & & 12.9 & 0.88 & .6 & 1.1 & & 1.1 & 0.80 & & 7.75 & 4.00 & - \\
\hline 2 & Co.029 & & 12.0 & 0.82 & 1.9 & 0.9 & & 1.9 & 0.83 & & 8.33 & 4.75 & 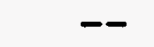 \\
\hline & $C 0.053$ & & 18.4 & 0.79 & & 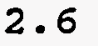 & & 1.5 & 0.80 & & 5.86 & 3.56 & 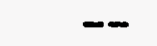 \\
\hline & $C 0.055$ & & 14.7 & 1.06 & 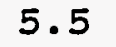 & 1. & & 1.4 & 78 & & 5.71 & 3.22 & - \\
\hline & Co. 044 & & 9.9 & 0.25 & & & & 2.2 & & & 7.33 & 4.00 & -- \\
\hline 3 & $\mathrm{CO} .060$ & & 13.5 & 0.94 & 4.3 & 1.8 & 92. & 1.5 & 0.77 & & 7.00 & 4.67 & \\
\hline
\end{tabular}

Note: Catalyst Run 9 screened thru $170 \times 400$ mesh.

Catalysts for Runs 11 thru 20 and Run 27 (except Runs 21 and 22)

screened thru $150 \times 400$ mesh.

Catalysts for Runs 21 and 22 screened thru $100 \times 400$ mesh.

(1) Weak TCD filaments in G.C. CH4 peaks smaller than expected.

(2) Internal valve in G.C. leaked. CH4 peak undetected. 
Table V

DATE: $12 / 30 / 94$

M3 SBCR RUN RESULTS

COMPARISON OF CATALYST ACTIVITY AT SIMIIAR RUN CONDITIONS

\begin{tabular}{lllc} 
Period No. & Temp. & Pres. & H2/CO Ratio \\
\hline 5 & $240 \mathrm{C}$ & $\frac{600 p s i}{1.0}$
\end{tabular}

a) Total flow is ca.15 I/min. STP, or $3 \mathrm{~cm} / \mathrm{sec}$ linear gas flow. Flows: N2-562 SLH, H2-II9 SLF, CO-II9 SLF

b) Conversion is total co conversion over the period ( $\left.\frac{\xi}{2}\right)$.

c) Conversion and selectivities are calculated using $\mathrm{N} 2$ as an internal standard in the GC analysis of the offgas.

d) Prod. rate: Rate for production of total hydrocarbons, Cl+ $(\mathrm{kg} / \mathrm{kg}$ cat., hr)

e) Alpha: Based on GC analysis of offgas and liquid product.

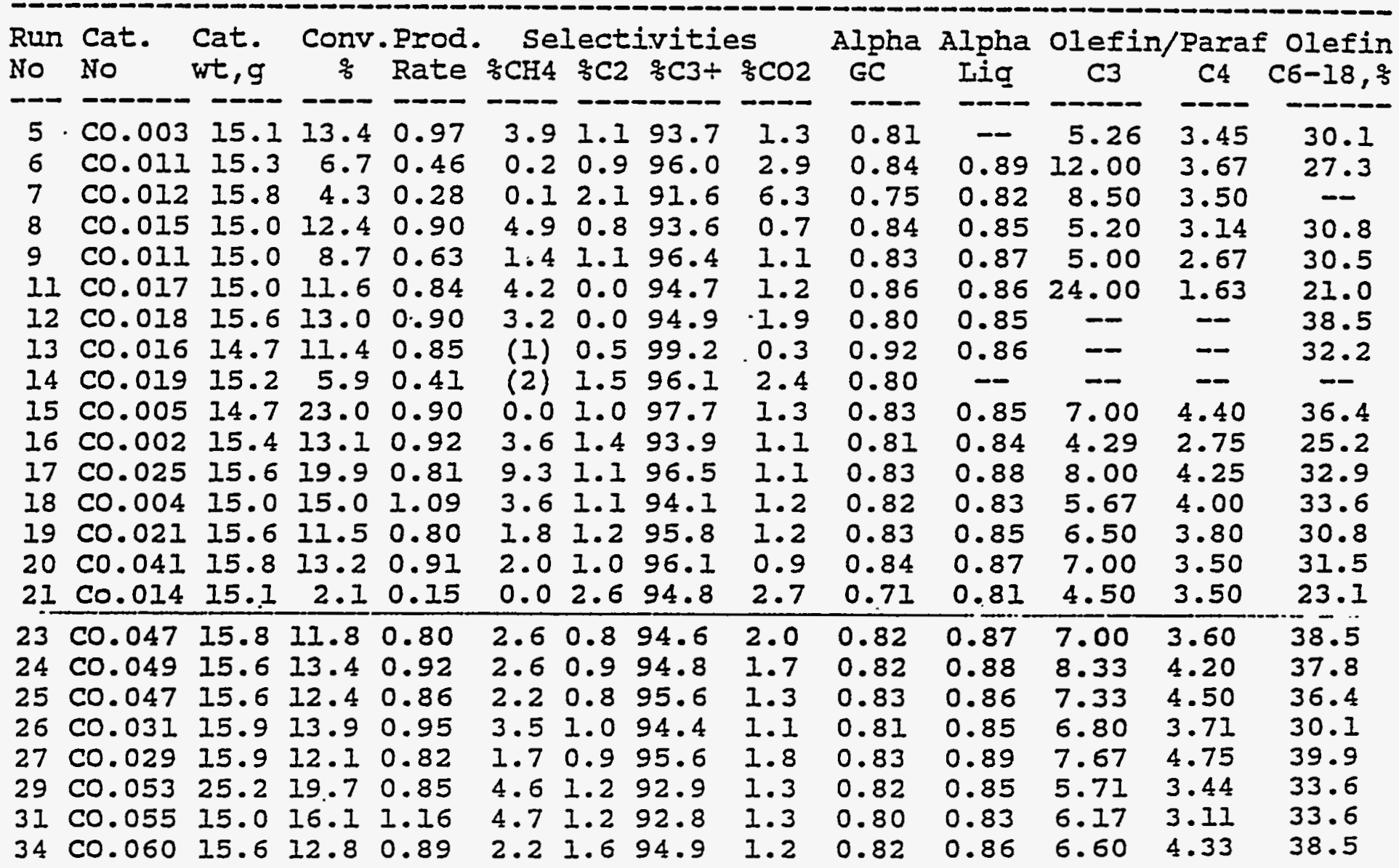

Note: Catalyst Run 9 screened thru $170 \times 400$ mesh.

Catalysts for Runs 11 thru 27 (except Runs 21 and 22)

screened thru $150 \times 400$ mesh.

Catalyst for Run 21 screened thru $100 \times 400$ mesh.

(1) Weak TCD filaments in G.C. CH4 peaks smaller than expected.

(2) Internal valve in G.C. leaked. CH4 peak undetected. 
SUMMARY OF H4 SBCR RUH RESULTS

a) Unless otheruise noted, total flow is ca.15 L/min. sip, or $3 \mathrm{~cm} / \mathrm{sec}$ linear gas flow.

b) Conversion is tocal co conversion over the period $(\%)$.

c)Conversion and selectivities are calculated using $\mathrm{HZ}$ as an internal

standard in the CC analysis of the offgas.

d)Prod. race: Race for production of total hydrocarbons, Cl+ (kg/kg cat., hr).

e)Alpha: Based on GC analysis of offgas and liquid product.

Run Cat. Cac. Per Time Time Temp Pres H2: Synfl Conv.Prod. Selec:ivities Alpha Algra Olefin/Paraf Olefins Conments

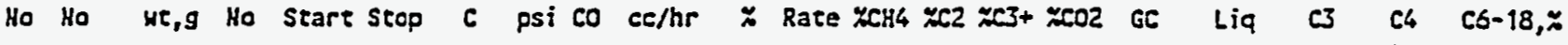

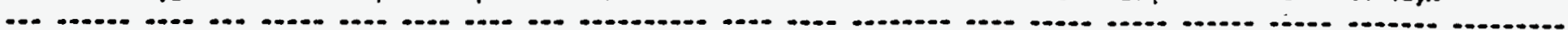

\begin{abstract}
\begin{tabular}{|c|c|c|c|c|c|}
\hline$c 0.00215 .2$ & 1 & 25 & 35 & 240 & 450 \\
\hline 360.00215 .2 & 2 & 73 & 83 & 220 & 450 \\
\hline 360.00215 .2 & 3 & 97 & 107 & 240 & 450 \\
\hline $300.002 \quad 15.2$ & 4 & 145 & 155 & 240 & 450 \\
\hline $60.002 \quad 15.2$ & 5 & 182 & 203 & 240 & 600 \\
\hline 60.00215 .2 & 6 & 217 & 226 & 240 & 450 \\
\hline Co.024 15.4 & 1 & 20 & 37 & 240 & 450 \\
\hline 60.02415 .4 & 2 & 68 & 85 & 220 & 450 \\
\hline$c 0.02415 .4$ & 3 & 92 & 109 & 240 & 450 \\
\hline$C 0.02415 .4$ & 6 & 140 & 157 & 240 & 450 \\
\hline $4 c 0.02415 .4$ & 5 & 188 & 205 & 240 & 600 \\
\hline 400.02415 .4 & 6 & 212 & 229 & 240 & 450 \\
\hline Co.035 15.4 & $i$ & 16 & 37 & 240 & 450 \\
\hline $\mathrm{C} 0.03515 .4$ & 2 & 68 & 85 & 220 & 450 \\
\hline Co.035 15.4 & 3 & 99 & 109 & 240 & 450 \\
\hline c0.035 15.6 & 4 & 136 & 157 & 240 & 450 \\
\hline .03515 .4 & 5 & 184 & 205 & 240 & 600 \\
\hline C0.035 15.4 & 6 & 212 & 228 & 240 & 450 \\
\hline
\end{tabular}
$600.02815 .4 \quad 1$ $600.02815 .4 \quad 2$ $600.02815 .4 \quad 3$ $600.02815 .4 \quad 4$ $600.02815 .4 \quad 5$ $600.028 \quad 15.4 \quad 6$

$\begin{array}{rrrrr}23 & 40 & 240 & 450 & 2.0 \\ 67 & 88 & 220 & 450 & 2.0 \\ 95 & 112 & 240 & 450 & 2.0 \\ 139 & 160 & 240 & 450 & 1.0 \\ 189 & 208 & 240 & 600 & 1.0 \\ 213 & 232 & 240 & 450 & 2.0\end{array}$

$\begin{array}{lllll}18 & 39 & 240 & 450 & 2.0\end{array}$ $\begin{array}{lllll}53 & 63 & 220 & 450 & 2.0\end{array}$ $94 \quad 111 \quad 240 \quad 4502.0$ $\begin{array}{lllll}138 & 159 & 240 \quad 450 & 1.0\end{array}$ $\begin{array}{lllll}191 & 207 & 240 & 600 & 1.0\end{array}$ $\begin{array}{lllll}215 & 231 & 240 & 450 & 2.0\end{array}$

$19 \quad 40 \quad 240 \quad 450 \quad 2.0 \quad 20$ $800.006 \quad 15.0 \quad 1$

700.04315 .4

7 c0.043 15.4

700.04315 .4

$7 \mathrm{C0.04315.4}$

$700.043 \quad 15.4$
\end{abstract}

$\begin{array}{rrrrrrrrrrrrr}20 & 29.4 & 1.42 & 17.3 & 2.2 & 79.4 & 1.01 & 0.73 & 0.82 & 0.91 & 0.60 & 17.5 & \\ 20 & 11.8 & 0.56 & 4.7 & 1.0 & 93.7 & 0.59 & 0.81 & 0.90 & 2.71 & 1.63 & -- & \text { G.C.Calib } \\ 20 & 26.4 & 1.28 & 16.0 & 3.1 & 80.1 & 0.88 & 0.73 & -. & 0.98 & 0.66 & -- & \\ 20 & 11.6 & 0.83 & 3.9 & 2.4 & 92.8 & 0.84 & 0.81 & 0.87 & 4.29 & 3.14 & \ldots- & \text { G.C.Calib } \\ 20 & 12.9 & 0.92 & 4.0 & 1.5 & 93.5 & 0.91 & 0.81 & 0.92 & 4.29 & 2.75 & 24.5 & \end{array}$

$\begin{array}{lllllllllllllllll}20 & 24.2 & 1.17 & 17.1 & 3.1 & 79.0 & 0.83 & 0.71 & \ldots & 1.03 & 0.70 & \ldots\end{array}$

$\begin{array}{rrrrrrrrrrrrr}20 & 25.5 & 1.21 & 8.6 & 2.0 & 89.0 & 0.43 & 0.79 & 0.84 & 2.93 & 1.93 & 21.7 & \\ 0 & 9.5 & 0.45 & 0.0 & 2.1 & 97.6 & 0.27 & 0.83 & 0.91 & 5.35 & 4.00 & \ldots & \text { Ho Synfl } \\ 0 & 23.7 & 1.13 & 9.5 & 2.1 & 88.0 & 0.45 & 0.77 & \ldots & 2.81 & 1.88 & \ldots & \\ 0 & 10.3 & 0.73 & 0.5 & 1.6 & 97.2 & 0.71 & 0.83 & 0.37 & 8.33 & 4.75 & \ldots & \\ 0 & 11.3 & 0.80 & 0.9 & 1.5 & 97.0 & 0.65 & 0.83 & 0.87 & 6.50 & 3.80 & 36.4 & \\ 0 & 21.5 & 1.02 & 9.3 & 2.3 & 87.9 & 0.50 & 0.76 & -- & 2.93 & 2.07 & \ldots & \\ 20 & 24.8 & 1.18 & 8.5 & 2.1 & 89.0 & 0.47 & 0.78 & 0.84 & 3.13 & 1.94 & 23.1 & \\ 20 & 8.8 & 0.42 & 0.0 & 2.3 & 97.4 & 0.31 & 0.82 & \ldots & 5.33 & 4.00 & \ldots- & \\ 20 & 23.5 & 1.11 & 8.7 & 2.1 & 88.6 & 0.61 & 0.77 & \ldots & 3.07 & 1.94 & \ldots & \\ 20 & 10.4 & 0.73 & 0.9 & 0.0 & 98.4 & 0.65 & -.- & 0.83 & -- & \ldots- & \ldots- & \text { G.C.Calib } \\ 20 & 10.7 & 0.76 & 0.7 & 1.4 & 97.1 & 0.75 & 0.84 & 0.88 & 6.25 & 4.50 & 27.3 & \\ 20 & 19.4 & 0.92 & 7.8 & 2.3 & 89.4 & 0.56 & 0.79 & \ldots & 3.08 & 1.77 & \ldots- & \end{array}$

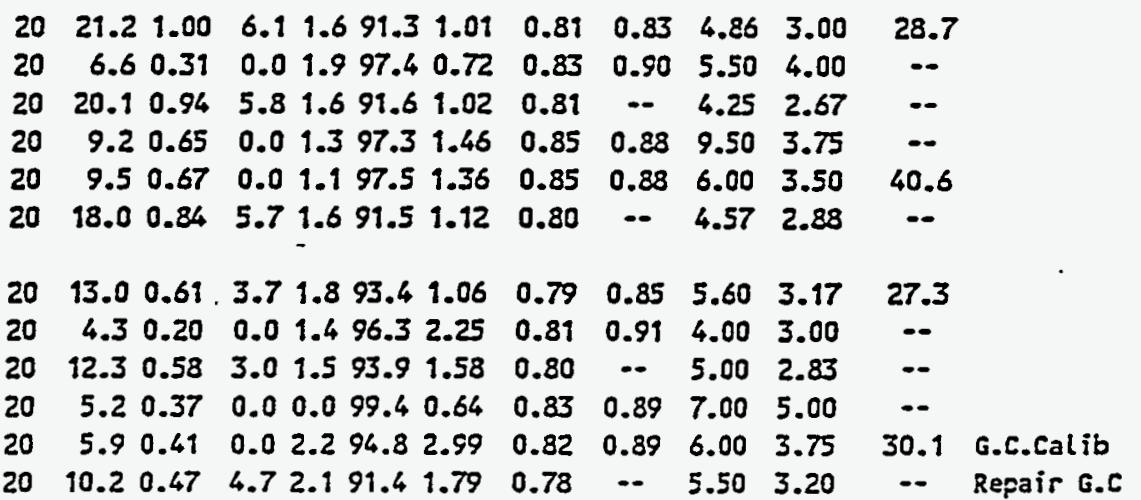

20

20

20

9.50 .45

23.71 .13

21.51 .02

20

10.20 .47

2.70 .13 
SUMMARY OF H4 SBCR RUN RESULTS

a) Unless otherwise noted, total flow is ca.15 L/min. STP, or $3 \mathrm{~cm} / \mathrm{sec}$ linear gas flow.

b) Conversion is total Co conversion over the period ( $\tilde{F}$ ).

e)Conversion and selectivities are calculated using $\mathrm{HZ}$ as an internal

standard in the $G E$ analysis of the offgas.

d) Prod. rate: Rate for production of total hydrocarbons, $c 1+(\mathrm{kg} / \mathrm{kg}$ cat., hr).

e)Alpha: Based on GC analysis of offgas and liquid produce.

Run Cat. Cat. Per Time Time Temp Pres H2: Synfl Conv.Prod. Selectivities Alpha Alpha Olefin/Paraf olefins Comments

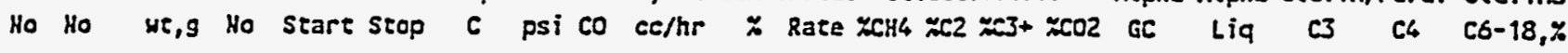

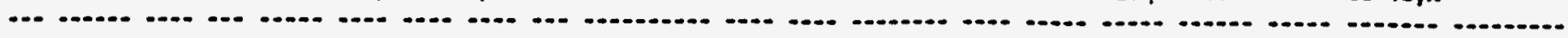

$\begin{array}{lllllllll}9 & 00.048 & 15.4 & 1 & 19 & 40 & 240 & 450 & 2.0 \\ 9 & 00.048 & 15.4 & 2 & 70 & 84 & 220 & 450 & 2.0 \\ 9 & 00.048 & 15.4 & 3 & 93 & 111 & 240 & 450 & 2.0 \\ 9 & 00.048 & 15.4 & 4 & 139 & 159 & 240 & 450 & 1.0 \\ 9 & 00.048 & 15.4 & 5 & 189 & 207 & 240 & 600 & 1.0 \\ 9 & 00.048 & 15.4 & 6 & 217 & 226 & 240 & 450 & 2.0\end{array}$

10 CAL.02 $15.9 \quad 1$ 10 CAL.02 $15.9 \quad 2$ 10 CAL.02 $15.9 \quad 3$ 10 CAL.02 $15.9 \quad 4$ 10 CAL.02 15.95 10 CAL.02 15.96

\begin{abstract}
$\begin{array}{lllll}19 & 40 \quad 240 \quad 450 \quad 2.0\end{array}$ $\begin{array}{llllll}69 & 88 & 220 & 450 & 2.0\end{array}$ $91 \quad 112 \quad 240 \quad 4502.0$ $139 \quad 160 \quad 240 \quad 4501.0$ $\begin{array}{lllll}187 & 201 & 240 & 600 & 1.0\end{array}$ $\begin{array}{lllll}215 & 230 & 240 & 450 & 2.0\end{array}$
\end{abstract}

11 CAL.03 $15.6 \quad 1$

$1200.05315 .9 \quad 1$ $12 \quad 0.053 \quad 15.9 \quad 2$ $1200.05315 .9 \quad 3$ 12 $00.053 \quad 15.9 \quad 4$ $12 \quad 0.053 \quad 15.9 \quad 5$ 12 C0.053 15.96

$13 \quad 00.05415 .7$ 13 c0.054 15.7 13 co.054 15.7 $13 \quad 0.054 \quad 15.7$ 13 00.05415 .7 $13 \quad 0.05415 .7$

14 stendA 20.01 14 BlendA 20.02 14 BlendA 20.03 14 BlendA $20.0 \quad 4$ 14 BlendA 20.05 14 BlendA 20.06

$\begin{array}{rrrrr}22 & 40 & 240 & 450 & 2.0 \\ 69 & 88 & 220 & 450 & 2.0 \\ 93 & 112 & 240 & 450 & 2.0 \\ 150 & 152 & 240 & 450 & 1.0 \\ 189 & 208 & 240 & 600 & 1.0 \\ 223 & 231 & 240 & 450 & 2.0\end{array}$

$\begin{array}{rrrrr}23 & 40 & 240 & 450 & 2.0 \\ 71 & 88 & 220 & 450 & 2.0 \\ 102 & 112 & 240 & 450 & 2.0 \\ 143 & 160 & 240 & 450 & 1.0 \\ 191 & 208 & 240 & 600 & 1.0 \\ 223 & 230 & 240 & 450 & 2.0 \\ & & & & \\ 25 & 39 & 240 & 450 & 2.0 \\ 69 & 87 & 220 & 450 & 2.0 \\ 93 & 111 & 240 & 450 & 2.0 \\ 143 & 158 & 240 & 450 & 1.0 \\ 191 & 207 & 240 & 600 & 1.0 \\ 215 & 231 & 240 & 450 & 2.0\end{array}$

$\begin{array}{lllll}215 & 231 & 240 & 450 & 2.0\end{array}$

$\begin{array}{rrrrrrrrrrrc}12 & 19.1 & 0.90 & 9.9 & 1.6 & 87.4 & 1.05 & 0.77 & 0.88 & 3.60 & 2.09 & 20.3 \\ 12 & 6.1 & 0.28 & 1.8 & 1.0 & 95.2 & 1.93 & 0.80 & 0.91 & 3.67 & 3.00 & \ldots \\ 20 & 17.8 & 0.84 & 10.1 & 1.7 & 86.9 & 1.33 & 0.77 & \ldots & 3.30 & 2.10 & \ldots \\ 20 & 8.2 & 0.57 & 2.3 & 1.0 & 93.3 & 2.98 & 0.82 & 0.90 & 8.50 & 4.33 & \ldots \\ 20 & 11.9 & 0.83 & 2.7 & 0.9 & 94.4 & 1.93 & 0.84 & 0.89 & 5.75 & 3.20 & 14.0 \\ 20 & 24.2 & 1.17 & 17.1 & 3.1 & 79.0 & 0.33 & 0.71 & \ldots & 1.03 & 0.70 & \ldots\end{array}$

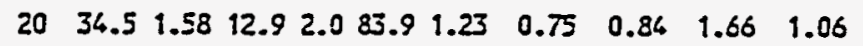
$\begin{array}{lllllllllll}20 & 14.9 & 0.68 & 4.6 & 0.9 & 96.0 & 0.47 & 0.81 & 0.88 & 4.40 & 2.29\end{array}$

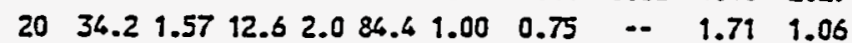
$\begin{array}{lllllllllll}20 & 14.6 & 1.00 & 4.6 & 1.1 & 93.3 & 0.97 & 0.80 & 0.88 & 7.20 & 4.00\end{array}$ $\begin{array}{lllllllllll}20 & 16.4 & 1.12 & 4.2 & 1.0 & 94.0 & 0.89 & 0.82 & 0.86 & 5.83 & 3.86\end{array}$ $\begin{array}{llllllllllll}20 & 30.9 & 1.42 & 13.3 & 2.1 & 83.7 & 0.90 & 0.74 & - & 1.90 & 1.21\end{array}$

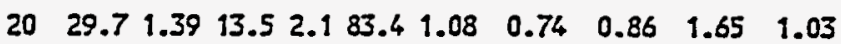

18.2 shutDosn

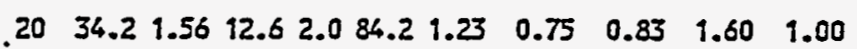
$\begin{array}{lllllllllll}20 & 14.4 & 0.66 & 4.3 & 1.0 & 94.3 & 0.48 & 0.81 & 0.89 & 4.20 & 2.67\end{array}$ $\begin{array}{lllllllllll}20 & 34.0 & 1.56 & 13.4 & 2.2 & 83.2 & 1.21 & 0.74 & - & 1.50 & 0.94\end{array}$ $\begin{array}{lllllllllll}20 & 13.3 & 0.91 & 4.3 & 1.2 & 93.3 & 1.18 & 0.80 & 0.89 & 6.60 & 3.71\end{array}$ $\begin{array}{lllllllllll}20 & 15.6 & 1.06 & 4.0 & 0.9 & 94.0 & 1.09 & 0.82 & 0.87 & 5.50 & 3.57\end{array}$ $\begin{array}{lllllllllll}20 & 29.8 & 1.37 & 12.8 & 2.0 & 84.3 & 0.94 & 0.75 & - & 1.89 & 1.10\end{array}$

21.0

$-$

$\cdots$

35.

35.7

-.

24.5

$--$

$-$

$-$

32.2

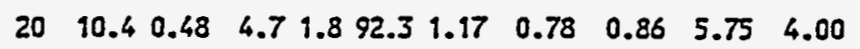

$\begin{array}{lllllllllll}20 & 0.2 & 0.01 & 0.0 & 19.60 .8 & 19.5 & 0.33 & 0.94 & 8.00 & 5.00\end{array}$

$\begin{array}{lllllllllll}20 & 10.6 & 0.49 & 4.5 & 1.5 & 92.8 & 1.23 & 0.79 & \ldots & 5.50 & 3.75\end{array}$

$\begin{array}{lllllllllll}20 & 5.2 & 0.36 & 0.0 & 1.2 & 97.0 & 1.85 & 0.83 & 0.95 & 6.00 & 4.50\end{array}$

20

5.00 .36

0.01 .396 .91 .78

$\begin{array}{llll}0.82 & 0.93 & 6.50 & 3.33\end{array}$

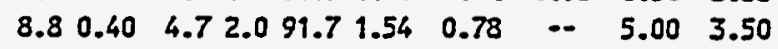

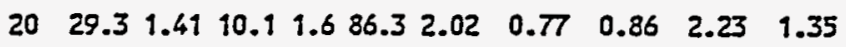
$\begin{array}{lllllllllll}20 & 12.4 & 0.60 & 4.3 & 0.9 & 93.9 & 0.95 & 0.81 & 0.91 & 3.80 & 2.33\end{array}$

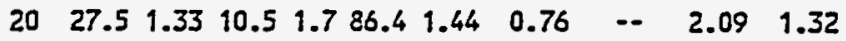
20

20

$\begin{array}{llllllllll}12.7 & 0.91 & 3.4 & 1.0 & 94.0 & 1.69 & 0.81 & 0.86 & 7.50 & 3.83\end{array}$

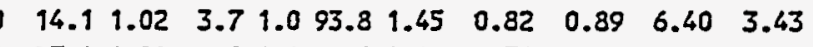

$20 \quad 27.01 .29 \quad 11.5 \quad 1.985 .5 \quad 1.12$

$\begin{array}{lllll}0.76 & \ldots & 2.04 & 1.30\end{array}$

25.9

--

--

30.8

30.8

23.1

$--$

--

-.

32.9 
a)Unless otherwise noted, total flow is ca.15 L/min. SiP. of $3 \mathrm{~cm} / \mathrm{sec}$ linear gas flow.

b)Conversion is total co conversion over the period ( $z$ ).

c) Conversion and selectivities are calculated using 12 as an intemal

standard in the GC analysis of the offgas.

d) Prod. rate: Rate for production of total hydrocarbons, C1+ (kg/kg cat.,hr).

e)Alpha: Based on $\mathscr{C}$ analysis of offgas and liquid product.

Run Cat. Cat. Per Iime Iime Iemp Pres E2: Synfl Conv.Prod. Selectivities Alpha Alpha Olefin/Paraf Olefins Corments Ho No wt,8 Ho Start Stop C psi CO ee/hF $z$ Rate zCH4 ZC2 ZC3+ zCO2 GC Liq C3 C4 C5-18,z

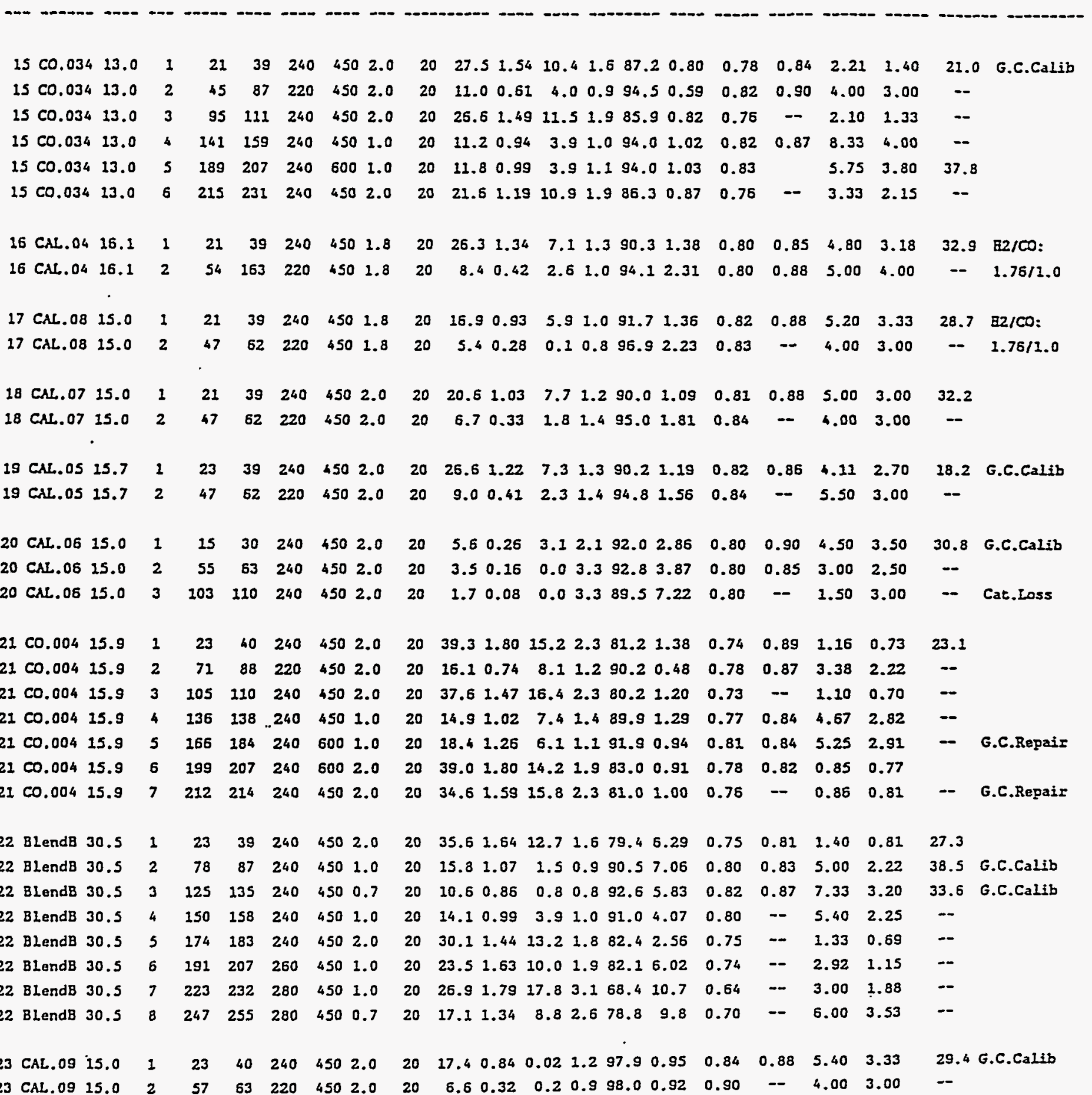


SURMARY OF MA SBCR RUN RESULTS

a) Unless otherwise noted, total flow is ca.15 L/min. STP, or $3 \mathrm{~cm} / \mathrm{sec}$ linear gas flow. b) Conversion is total co conversion over the period ( $z$ ).

c)Conversion and selectivities are calculated using $\mathrm{k2}$ as an internal

standard in the EC analysis of the offgas.

d) Prod. rate: Rate for production of total hydrocarbons, Cl+ (kg/kg cat., hr).

-)Alpha: Based on GC analysis of offgas and liquid product.

Run Cat. Cat. Per TIme Time Teap Pres a2: Synfl Conv.Prod. Selectivities Alpha Alpha Olefin/Paraf Olefins Coments

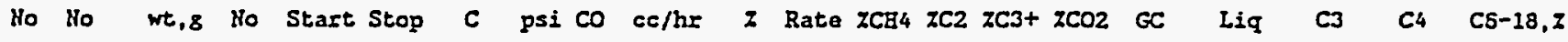
Wo

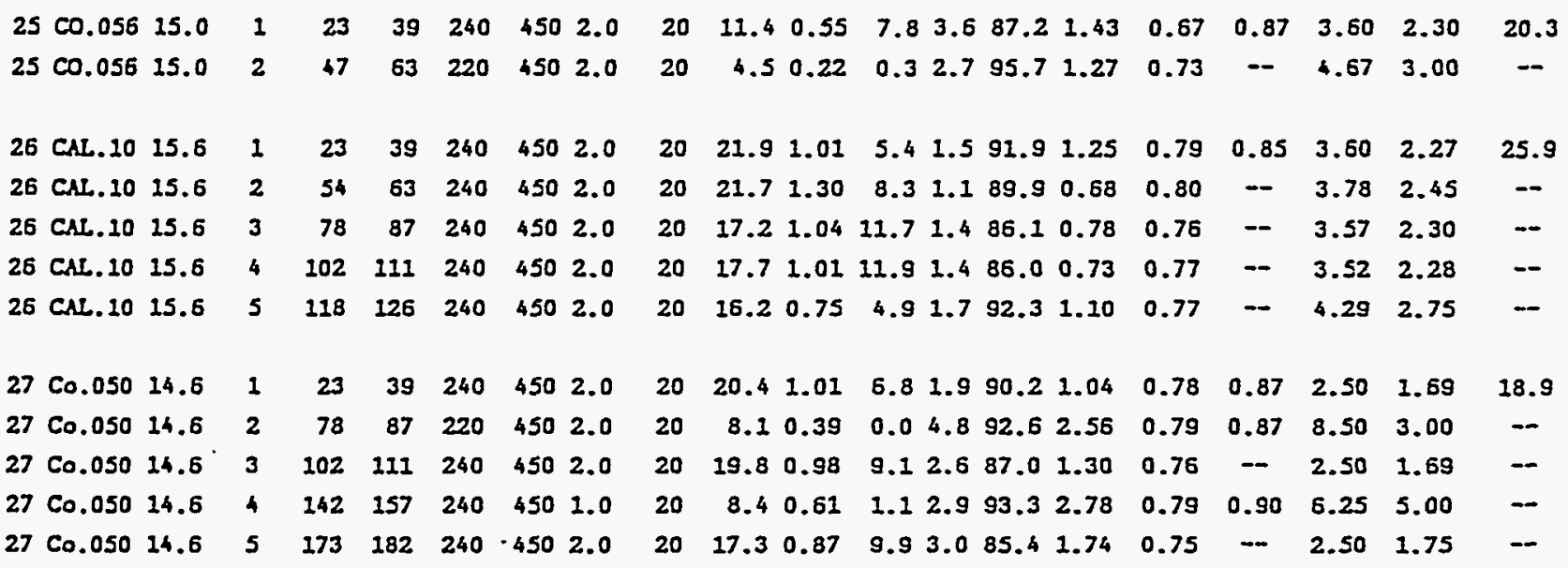


Table VII

DATE: $12 / 30 / 94$

M4 SBCR RUN RESULTS

COMPARISON OF CATAIYST ACTIVITY AT SIMIIAR RUN CONDITIONS

\begin{tabular}{cccc} 
Period No. & Temp. & Pres. & H2/CO Ratio \\
\hline$I$ & $240 \mathrm{C}$ & $450 \mathrm{psi}$ & 2.0
\end{tabular}

a) Total flow is ca.15 I/min. STP, or $3 \mathrm{~cm} / \mathrm{sec}$ Iinear gas flow. Flows:N2-563 SIIE, H2-225 SLIH, CO-II2.5 SLH

b) Conversion is total $C O$ conversion over the period (\%).

c) Conversion and selectivities are calculated using $\mathrm{N2}$ as an internal standard in the GC analysis of the offgas.

d) Prod. rate: Rate for production of total hydrocarbons $\mathrm{Cl} \div$ (kg/kg cat., hr). e) Alpha: Based on GC analysis of offgas and Iiquid product.

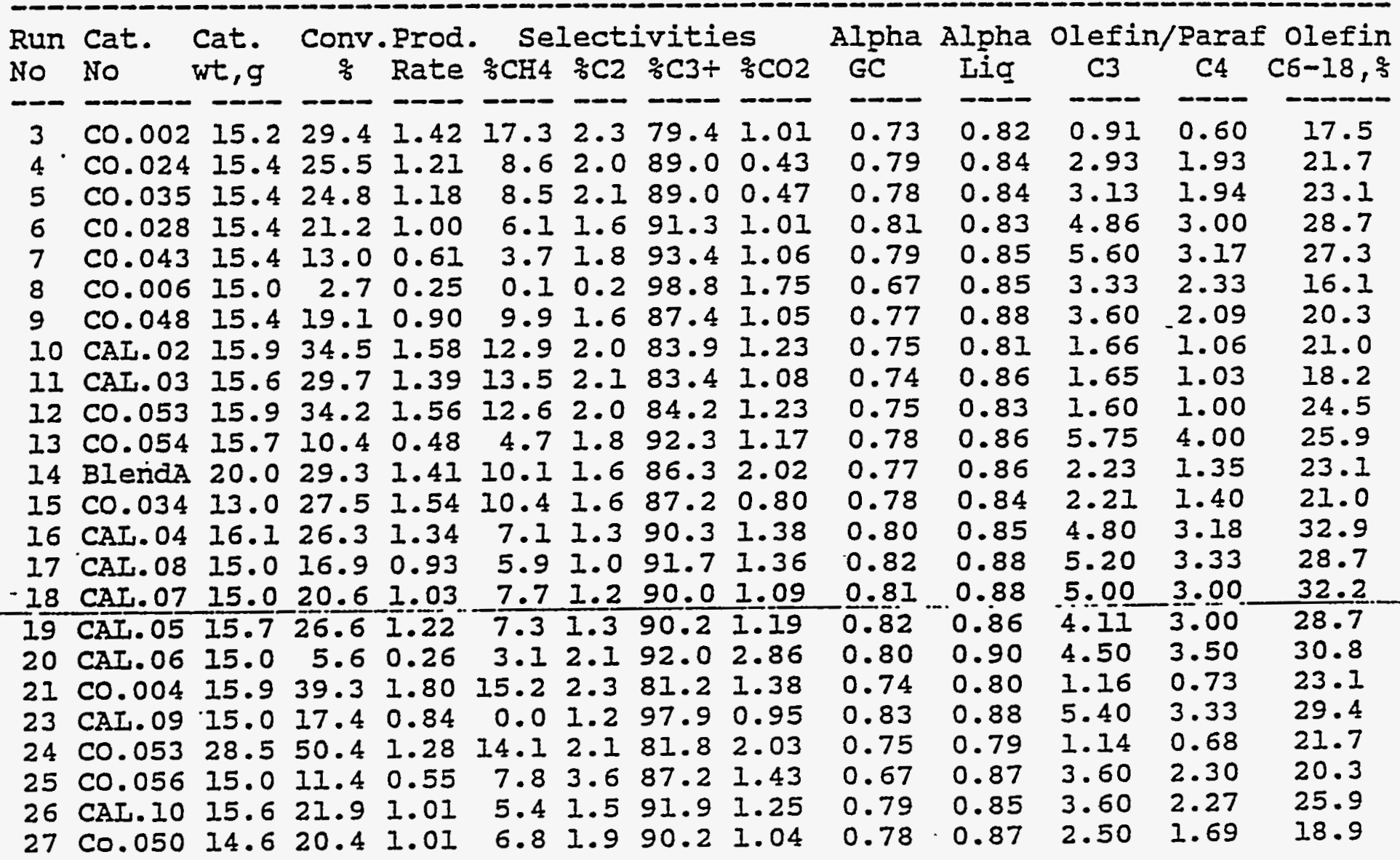

Note: Catalysts for Runs 3 thru 15 (except 8) screened through $150 \times 400$ mesh.

Catalyst for Run 8 screened thru $100 \times 400$ mesh.

Cat. NOS. CAI.08 and CAI.07 were prereduced and coated with wax.

H2 to $C O$ ratio for Runs 16 and 17 was $1.76 / 1.0$.

Cat. No. CAL.06 was $\mathrm{H2}$ reduced and air stabilized.

Cat. No. CAI.09 was prereduced and coated with soya. 
Table VIII

DATE: $12 / 30 / 94$

M4 SBCR RUN RESULTS

COMPARISON OF CATAIYST ACTIVITY AT SIMILAR RUN CONDITIONS

\begin{tabular}{cccc} 
Period No. & Temp. & Pres. & H2/CO Ratio \\
\hline 2 & $220 \mathrm{C}$ & $450 \mathrm{psi}$ & 2.0
\end{tabular}

a) Total flow is ca.15 L/min. STP, or $3 \mathrm{~cm} / \mathrm{sec}$ linear gas flow. Flows:N2-563 SLH, H2-225 SLH, CO-II2.5 SLF

b) Conversion is total co conversion over the period ( $\left.\frac{f}{b}\right)$.

c) Conversion and selectivities are calculated using $\mathrm{N} 2$ as an internal standard in the GC analysis of the offgas.

d) Prod. rate: Rate for production of total hydrocarbons $\mathrm{Cl} \div(\mathrm{kg} / \mathrm{kg} \mathrm{cat.}, \mathrm{hr})$. e) Alpha: Based on GC analysis of offgas and liquid product.

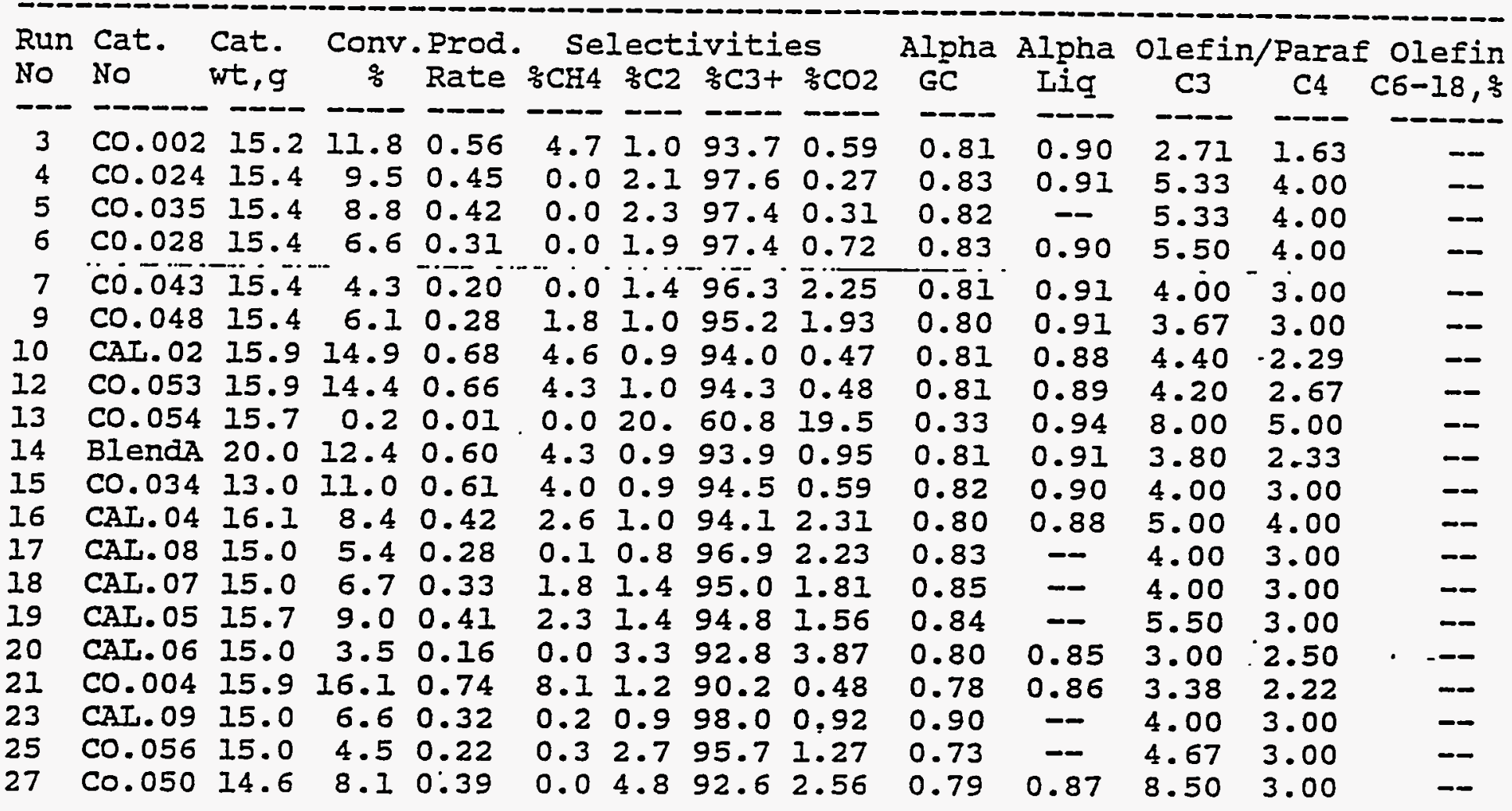

Note: Catalysts for Runs 3 through 15 screened thru $150 \times 400$ mesh. Blend A contains $15.0 \mathrm{gm}$ of Cat. No. CO.005 plus $5.0 \mathrm{gm}$ of Cat. No. WGS.03.

Cat. NOs. CAL.08 and CAL.07 were prereduced and wax coated. H2 to CO for Runs 16 and 17 was $1.76 / 1.0$.

Cat. No. CAI. 06 was H2 reduced and air stabilized before charging. Cat. No. CAL.09 was prereduced and coated with Soya. 
M4 SBCR RUN RESULTS

COMPARISON OF CATALYST ACTIVITY AT SIMIIAR RUN CONDITIONS

\begin{tabular}{llll} 
Period No. & Temp. & Pres. & F2/CO Ratio \\
\hline 4 & $240 \mathrm{C}$ & $450 \mathrm{psi}$ & 1.0
\end{tabular}

a) Total flow is ca.15 I/min. STP, or $3 \mathrm{~cm} / \mathrm{sec}$ linear gas flow. Flows: N2-562 SLH, H2-119 SLH, CO-119 SIH

b) Conversion is total co conversion over the period ( $\%$ ).

c) Conversion and selectivities are calculated using $N 2$ as an internal standard in the GC analysis of the offgas.

d) Prod. rate: Rate for production of total hydrocarbons $\mathrm{cl}+$ (kg/kg cat., hr). e) Alpha: Based on GC analysis of offgas and liquid product.

\begin{tabular}{|c|c|c|c|c|c|c|c|c|c|c|c|c|c|}
\hline${ }_{0}^{u n}$ & $\begin{array}{l}\text { Cat. } \\
\text { No }\end{array}$ & $\begin{array}{l}\text { Cat. } \\
\text { wt, g }\end{array}$ & $\underset{\%}{\text { Conv. }}$ & $\begin{array}{l}\text { Prod. } \\
\text { Rate }\end{array}$ & $\begin{array}{c}\mathrm{SE} \\
\% \mathrm{CH} 4 \\
\end{array}$ & & ie & $\begin{array}{l}\text { es } \\
\% \mathrm{CO} 2\end{array}$ & $\begin{array}{l}\text { Alpha } \\
\text { GC }\end{array}$ & $\begin{array}{l}\text { Alpha } \\
\text { Iig }\end{array}$ & $\begin{array}{r}\text { Olef } \\
\text { C3 }\end{array}$ & $\begin{array}{l}\text { Paraf } \\
\text { C4 }\end{array}$ & 0 \\
\hline $\begin{array}{l}5 \\
6 \\
7\end{array}$ & $\begin{array}{l}C 0.002 \\
C 0.024 \\
C 0.035 \\
C 0.028 \\
C 0.043 \\
C 0.048 \\
C A L .02 \\
C 0.053 \\
C 0.054 \\
B I \text { endA } \\
C 0.034 \\
C 0.004\end{array}$ & $\begin{array}{l}15.2 \\
15.4 \\
15.4 \\
15.4 \\
15.4 \\
15.4 \\
15.9 \\
15.9 \\
15.7 \\
20.0 \\
13.0 \\
15.9\end{array}$ & $\begin{array}{r}11.6 \\
10.3 \\
10.4 \\
9.2 \\
5.2 \\
8.2 \\
14.6 \\
13.3 \\
5.2 \\
12.7 \\
11.2 \\
14.9\end{array}$ & $\begin{array}{l}0.83 \\
0.73 \\
0.73 \\
0.65 \\
0.37 \\
0.57 \\
1.00 \\
0.91 \\
0.36 \\
0.91 \\
0.94 \\
1.02\end{array}$ & $\begin{array}{l}3.9 \\
0.5 \\
0.9 \\
0.0 \\
0.0 \\
2.3 \\
4.6 \\
4.3 \\
0.0 \\
3.4 \\
3.9 \\
7.4\end{array}$ & $\begin{array}{l}0 \\
1 \\
1 \\
1 \\
1 \\
1 \\
1 \\
1 .\end{array}$ & $\begin{array}{l}92.8 \\
97.2 \\
98.4 \\
97.3 \\
99.4 \\
93.8 \\
93.3 \\
93.3 \\
97.0 \\
94.0 \\
94.0 \\
89.9\end{array}$ & $\begin{array}{l}0.84 \\
0.71 \\
0.65 \\
1.46 \\
0.64 \\
3.00 \\
0.97 \\
1.18 \\
1.85 \\
1.61 \\
1.02 \\
1.29\end{array}$ & $\begin{array}{l}0.81 \\
0.83 \\
-- \\
0.85 \\
0.83 \\
0.82 \\
0.80 \\
0.80 \\
0.83 \\
0.81 \\
0.82 \\
0.77\end{array}$ & $\begin{array}{l}7 \\
7 \\
3 \\
8 \\
9 \\
0 \\
8 \\
9 \\
5 \\
6\end{array}$ & $\begin{array}{l}4.29 \\
8.33 \\
-1.50 \\
9.50 \\
7.00 \\
8.50 \\
7.20 \\
6.60 \\
6.00 \\
7.50 \\
8.33 \\
4.67\end{array}$ & $\begin{array}{c}3.14 \\
4.75 \\
-2 \\
3.75 \\
5.00 \\
4.33 \\
4.00 \\
3.71 \\
4.50 \\
3.83 \\
4.00 \\
2.82\end{array}$ & $\begin{array}{l}- \\
- \\
- \\
- \\
- \\
-- \\
-- \\
--\end{array}$ \\
\hline
\end{tabular}

Note: Catalysts for Runs 3 through 21 screened thru $150 \times 400$ mesh. 
Table $\mathrm{X}$

DATE: $12 / 30 / 94$

M4 SBCR RUN RESULTS

COMPARISON OF CATALYST ACTIVITY AT SIMIIAR RUN CONDITIONS

\begin{tabular}{cccc} 
Period No. & Temp. & Pres. & H2/CO Ratio \\
\hline 5 & $240 \mathrm{C}$ & $600 \mathrm{psi}$ & 1.0
\end{tabular}

a) Total flow is ca.I5 $\mathrm{I} / \mathrm{min}$. STP, or $3 \mathrm{~cm} / \mathrm{sec}$ Iinear gas flow.

b) Conversion is total co conversion over the period $\left(\frac{s}{3}\right)$.

Flows: N2-562 SIF, F2-II9 SIF, CO-IIS SIH

c) Conversion and selectivities are calculated using $\mathrm{N} 2$ as an internal standard in the GC analysis of the offgas.

d) Prod. rate: Rate for production of total hydrocarbons Cl+ (kg/kg cat.,hr).

e) Alpha: Based on GC analysis of offgas and liquid product.

\begin{tabular}{|c|c|c|c|c|c|c|c|c|c|c|c|c|c|}
\hline $\begin{array}{l}\text { Run } \\
\text { No }\end{array}$ & $\begin{array}{l}\text { Cat. } \\
\text { No }\end{array}$ & $\begin{array}{l}\text { cat. } \\
\text { wt, g }\end{array}$ & $\begin{array}{c}\text { Conv. } \\
\frac{q}{5}\end{array}$ & $\begin{array}{l}\text { Prod. } \\
\text { Rate }\end{array}$ & $\begin{array}{r}\text { SeI } \\
5 \mathrm{CH}^{2}\end{array}$ & $\begin{array}{l}\text { Iecti } \\
\text { \&c } 2\end{array}$ & $\begin{array}{l}\text { ivitie } \\
\text { sc3 } \div\end{array}$ & $\begin{array}{l}\text { es } \\
\stackrel{5}{ } \mathrm{CO} 2\end{array}$ & $\begin{array}{l}\text { Alpha } \\
\text { GC }\end{array}$ & $\begin{array}{l}\text { Alpina } \\
\text { Iig }\end{array}$ & $\begin{array}{r}\text { Ief: } \\
\text { c3 }\end{array}$ & $\begin{array}{l}\text { Paraf } \\
\text { C4 }\end{array}$ & $\begin{array}{l}\text { olefin } \\
\text { cs-18, }\end{array}$ \\
\hline $\begin{array}{r}3 \\
4 \\
5 \\
6 \\
7 \\
9 \\
10 \\
12 \\
13 \\
14 \\
15 \\
21 .\end{array}$ & $\begin{array}{l}C 0.002 \\
C 0.024 \\
C 0.035 \\
C 0.028 \\
C 0.043 \\
C 0.048 \\
C A I .02 \\
C 0.053 \\
C 0.054 \\
B 1 \text { endA } \\
C 0.034 \\
C 0.004\end{array}$ & $\begin{array}{l}15.2 \\
15.4 \\
15.4 \\
15.4 \\
15.4 \\
15.4 \\
15.9 \\
15.9 \\
15.7 \\
20.0 \\
13.0 \\
15.9\end{array}$ & $\begin{array}{r}12.9 \\
11.3 \\
10.7 \\
9.5 \\
5.9 \\
11.9 \\
16.4 \\
15.6 \\
5.0 \\
14.1 \\
11.8 \\
18.4\end{array}$ & $\begin{array}{l}0.92 \\
0.80 \\
0.76 \\
0.67 \\
0.41 \\
0.83 \\
1.12 \\
1.06 \\
0.34 \\
1.02 \\
0.99 \\
1.26\end{array}$ & $\begin{array}{l}4.0 \\
0.9 \\
0.7 \\
0.0 \\
0.0 \\
2.7 \\
4.2 \\
4.0 \\
0.0 \\
3.7 \\
3.9 \\
6.1\end{array}$ & $\begin{array}{l}1.5 \\
1.5 \\
1.4 \\
1.1 \\
2.2 \\
0.9 \\
1.0 \\
0.9 \\
1.3 \\
1.0 \\
1.1 \\
1.1\end{array}$ & $\begin{array}{l}93.5 \\
97.0 \\
97.1 \\
97.5 \\
94.8 \\
94.4 \\
94.0 \\
94.0 \\
96.9 \\
93.8 \\
94.0 \\
91.9\end{array}$ & $\begin{array}{l}0.91 \\
0.65 \\
0.75 \\
1.36 \\
2.99 \\
1.93 \\
0.89 \\
1.0 \\
1.78 \\
1.45 \\
1.03 \\
0.94\end{array}$ & $\begin{array}{l}0.81 \\
0.83 \\
0.84 \\
0.85 \\
0.82 \\
0.84 \\
0.82 \\
0.82 \\
0.82 \\
0.82 \\
0.83 \\
0.81\end{array}$ & $\begin{array}{l}0.82 \\
0.87 \\
0.88 \\
0.88 \\
0.89 \\
0.89 \\
0.86 \\
0.87 \\
0.93 \\
0.89 \\
0.87 \\
0.84\end{array}$ & $\begin{array}{l}4.29 \\
6.50 \\
6.25 \\
6.00 \\
6.00 \\
5.75 \\
5.83 \\
5.50 \\
6.50 \\
6.40 \\
5.75 \\
5.25\end{array}$ & $\begin{array}{l}2.75 \\
3.80 \\
4.50 \\
3.50 \\
3.75 \\
3.20 \\
3.86 \\
3.57 \\
3.33 \\
3.43 \\
3.80 \\
2.91\end{array}$ & $\begin{array}{l}24.5 \\
36.4 \\
27.3 \\
40.6 \\
30.7 \\
14.0 \\
35.7 \\
32.2 \\
30.8 \\
32.9 \\
37.8 \\
32.2\end{array}$ \\
\hline
\end{tabular}

Note: Catalysts for Runs 3 through 21 screened thru $150 \times 400$ mesh. 